\title{
Asymmetric Mannich Reaction of Isatin-Based Ketimines with $\alpha$-Diazomethylphosphonates Catalyzed by Chiral Silver Phosphate
}

\author{
Jing Chen, Xiaojing Wen, Yan Wang, Fei Du, Liu Cai and Yungui Peng *
}

Key Laboratory of Applied Chemistry of Chongqing Municipality, School of Chemistry and Chemical Engineering, Southwest University, Chongqing 400715, People's Republic of China

\section{Table of Contents}

1. General information.

2. Synthesis of chiral phosphoric acid (6k and 6l).

3. Optimization of the reaction conditions

4. General procedure for the asymmetric Mannich reaction

5. Characterization of the Mannich products 4

6. Hydrogenation of (S)-tert-butyl (3-(diazo(dimethoxyphosphoryl)methyl)-1-methyl-2-oxoindolin-3yl)carbamate (4aaa) into 1a.

7. Synthesis of (S)-tert-butyl (3-(dimethoxyphosphoryl)-4-(2-(methylamino)phenyl)-5-oxo-4,5dihydro-1H-pyrazol-4-yl)carbamate 9.

8. X-Ray crystallographic analysis of 4aaa and determination of the absolute configurations

9. Proposed Mechanism.

10. References

11. NMR spectra for chiral phosphoric acids and products 


\section{General information}

All solvents were purified by standard procedures and distilled prior to use. Reagents obtained from commercial source were used without further purification. Petroleum ether and ethyl acetate for flash column chromatography were distilled before use. All reactions were monitored by TLC with silica gel coated plates. Flash column chromatography was performed on silica gel $\mathrm{H}(10-40 \mu)$. NMR spectra were recorded on Bruker Avance $600 \mathrm{MHz}$ instruments. Chemical shifts (d) are given in ppm relative to TMS, coupling constants $(\mathcal{J})$ in $\mathrm{Hz}$. Optical rotations were measured on a Perkin Elmer 341 Polari meter at $\lambda=589 \mathrm{~nm}$. Analytical high performance liquid chromatography (HPLC) was carried out on WATERS 510 instrument (2487 Dual $\lambda$ Absorbance Detector and 515 HPLC Pump) using chiral column. ESI HRMS was recorded on a Waters SYNAPT G2. Isatin-derived ketimines, ${ }^{1} \alpha$-diazo compounds ${ }^{2}$ and chiral $^{2}$ phosphoric acid $\mathbf{6 a}-\mathbf{6} \mathbf{j},{ }^{3 \mathrm{a}} 7 \mathbf{a}^{3 \mathrm{~b}}$ and $\mathbf{7 \mathbf { b } ^ { 3 a }}$ were prepared according to the literature.

\section{Synthesis of chiral phosphoric acid (6k and 6I)}

\subsection{The catalyst $6 \mathrm{k}$ was prepared according to the literature. ${ }^{4}$}<smiles>CC(C)c1cc(-c2cccc3ccccc23)cc(C(C)C)c1-c1cc2ccccc2c(-c2c(O)c(-c3c(C(C)C)cc(-c4cccc5ccccc45)cc3C(C)C)cc3ccccc23)c1O</smiles>

10<smiles>CC(C)c1cc(-c2cccc3ccccc23)cc(F)c1-c1cc2ccccc2c(-c2c(OP(=O)(O)O)c(-c3c(C(C)C)cc(-c4cccc5ccccc45)cc3C(C)C)cc3ccccc23)c1OP(=O)(O)O</smiles>

$6 \mathbf{k}$

To a solution of diol $10(0.50 \mathrm{~g}, 0.582 \mathrm{mmol})$ in pyridine $(5 \mathrm{~mL})$ at $0{ }^{\circ} \mathrm{C}$ was added $\mathrm{POCl}_{3}(0.1 \mathrm{~mL}, 1.048 \mathrm{mmol})$. The resultant mixture was heated at $90^{\circ} \mathrm{C}$ for $12 \mathrm{~h}$. Upon cooling down to ambient temperature and water $(1.0 \mathrm{~mL})$ was added, followed by heating to $90{ }^{\circ} \mathrm{C}$ for $4 \mathrm{~h}$. Then $6 \mathrm{M} \mathrm{HCl}(5 \mathrm{~mL})$ was added to the mixture. Heating was continued for an additional $5 \mathrm{~h}$. The reaction was extracted with $\mathrm{CH}_{2} \mathrm{Cl}_{2}$ twice. The organic phase was dried $\left(\mathrm{Na}_{2} \mathrm{SO}_{4}\right)$ and concentrated. The residue was purified by silica gel chromatography (eluting with petroleum ether/EtOAc $=5 / 1$ to 1/1). The obtained product was re-dissolved in $\mathrm{CH}_{2} \mathrm{Cl}_{2}(5 \mathrm{~mL})$ and $6 \mathrm{M}$ aqueous $\mathrm{HCl}(5 \mathrm{~mL})$, and the reaction mixture was stirred for $2.0 \mathrm{~h}$ at ambient temperature. The organic layer was separated, dried over anhydrous $\mathrm{Na}_{2} \mathrm{SO}_{4}$, filtered and concentrated in vacuo. The residue was evaporated to dryness and dried under high vacuum to give white solid 6k (0.455 g, 85\%). ${ }^{1} \mathrm{H}$ NMR $\left(600 \mathrm{MHz}, \mathrm{CDCl}_{3}\right) \delta 7.96$ (d, $\left.J=7.1 \mathrm{~Hz}, 6 \mathrm{H}\right), 7.81$ (d, $\left.J=31.0 \mathrm{~Hz}, 4 \mathrm{H}\right), 7.53(\mathrm{~d}, J=5.6$ $\mathrm{Hz}, 2 \mathrm{H}), 7.38$ (d, J = 19.9 Hz, 6H), 7.34-7.24 (m, 6H), $7.23(\mathrm{~s}, 4 \mathrm{H}), 2.69$ (d, J = 30.0 Hz, 4H), 1.07 (d, J = 44.8 Hz, $12 \mathrm{H}), 0.91(\mathrm{~s}, 7 \mathrm{H})$, $0.76(\mathrm{~s}, 5 \mathrm{H}) .{ }^{13} \mathrm{C}$ NMR $\left(151 \mathrm{MHz}, \mathrm{CDCl}_{3}\right) \delta 148.2,147.6,145.8,145.8,140.9,140.5,133.8,132.7,132.4,132.0,131.7,131.1,128.2$, 128.1, 127.4, 126.9, 126.4, 126.3, 126.0, 125.8, 125.6, 125.3, 125.2, 124.2, 122.1, 31.1, 30.8, 26.4, 24.9, 23.3, 22.9 ppm. HRMS (ESI-TOF) calcd. for $\mathrm{C}_{64} \mathrm{H}_{57} \mathrm{O}_{4} \mathrm{P}[\mathrm{M}+\mathrm{Na}]^{+}$: 943.3892, found: 943.3897. [a] $]_{0}^{20}=-87.3^{\circ}\left(\mathrm{c}=1.0, \mathrm{CH}_{2} \mathrm{Cl}_{2}\right)$.

\subsection{Synthesis of the catalyst $6 \mathrm{I}$.}




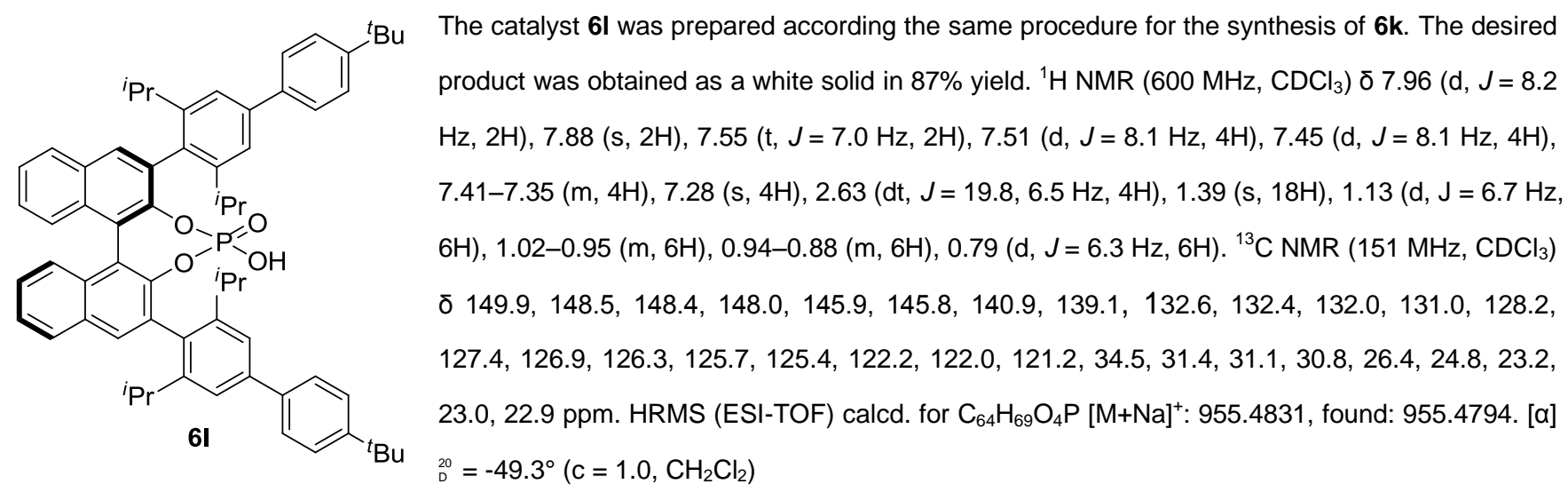

\section{Optimization of the reaction conditions}

3.1 The effects of temperature and solvents on the nucleophile addition between imine isatin 3aa and $\alpha$ diazomethylphosphonate $2 a$.

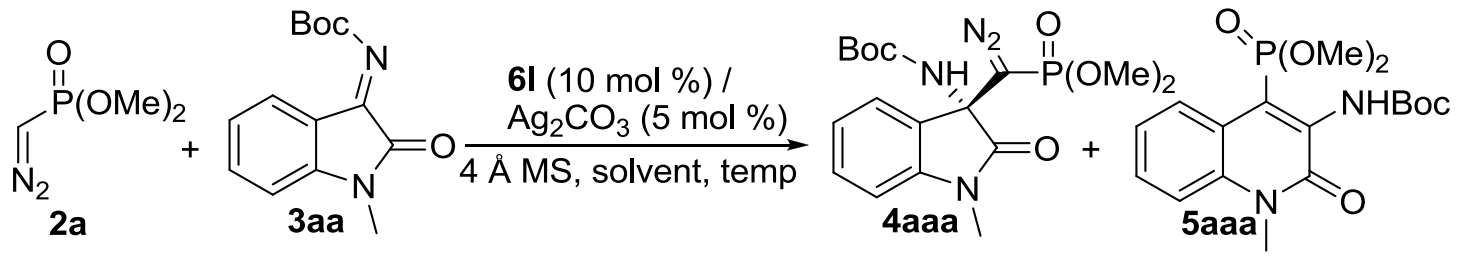

\begin{tabular}{|c|c|c|c|c|c|c|}
\hline entry $^{a}$ & Temp $\left({ }^{\circ} \mathrm{C}\right)$ & solvent & time $(h)$ & $\begin{array}{c}\text { yield of } \\
4 a a a(\%)^{b}\end{array}$ & $e e(\%)^{c}$ & $\begin{array}{c}\text { yield of } \\
5 \text { aaa }(\%)^{b}\end{array}$ \\
\hline 1 & -20 & Tol & 35 & 49 & 99 & 31 \\
\hline 2 & -30 & Tol & 60 & 80 & 98 & 15 \\
\hline 3 & -40 & Tol & 144 & 61 & 98 & 3 \\
\hline $4^{d}$ & -30 & Tol & 24 & 73 & 93 & 18 \\
\hline $5^{e}$ & -30 & Tol & 34 & 80 & 96 & 13 \\
\hline $6^{\dagger}$ & -30 & Tol & 120 & 61 & 96 & 17 \\
\hline 7 & -30 & DCM & 60 & 20 & 98 & 15 \\
\hline 8 & -30 & CPME & 60 & trace & - & - \\
\hline 9 & -30 & THF & 60 & trace & - & - \\
\hline 10 & -30 & hexane & 60 & $\mathrm{nr}$ & - & - \\
\hline 11 & -30 & ${ }^{i} \mathrm{PrOH}$ & 60 & 99 & 20 & - \\
\hline
\end{tabular}




\begin{tabular}{|c|c|c|c|c|c|c|}
\hline 12 & -30 & $\mathrm{CH}_{3} \mathrm{CN}$ & 60 & 75 & 0 & 3 \\
\hline 13 & -30 & PhEt & 60 & 78 & 98 & 5 \\
\hline 14 & -30 & $\mathrm{PhF}$ & 60 & 75 & 98 & 24 \\
\hline 15 & -30 & mesitylene & 60 & 75 & 78 & 10 \\
\hline 16 & -30 & methylcyclohexane & 60 & 76 & 82 & 14 \\
\hline 17 & -30 & Tol/PhEt (1:1) & 60 & 95 & 98 & 5 \\
\hline 18 & -30 & Tol/PhEt (1:2) & 60 & 85 & 99 & 5 \\
\hline 19 & -30 & Tol/PhEt (2:1) & 60 & 88 & 99 & 0 \\
\hline
\end{tabular}

${ }^{a}$ Performed under argon with 2a $(0.1 \mathrm{mmol})$, 3aa $(0.11 \mathrm{mmol}), 4 \AA \mathrm{MS}(50 \mathrm{mg}), 6 \mathrm{l}(10 \mathrm{~mol} \%)$ and $\mathrm{Ag}_{2} \mathrm{CO}_{3}(5 \mathrm{~mol} \%)$ in $1.0 \mathrm{~mL}$ of solvent at $-30{ }^{\circ} \mathrm{C}$. ${ }^{b}$ Isolated yield. ${ }^{c}$ Determined by HPLC analysis. ${ }^{d}$ Using $4.0 \mathrm{~mL}$ of toluene at $-30{ }^{\circ} \mathrm{C}$. ${ }^{e}$ Using $2.0 \mathrm{~mL}$ of toluene at $30{ }^{\circ} \mathrm{C}$. Using $0.5 \mathrm{~mL}$ of toluene at $-30^{\circ} \mathrm{C}$. CPME= Cyclopentyl Methyl Ether

\subsection{Investigation the influence of the substituent at the $\mathrm{N} 1$ position of the isatin-derived $\mathrm{N}$-Boc ketimines}

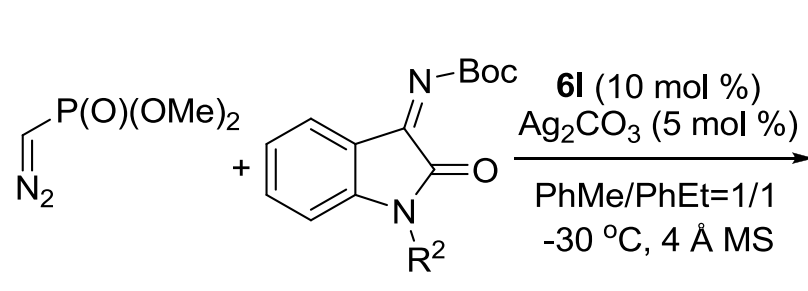

$2 a$<smiles>[R2]N1C(=O)[C@]2(C(=N)P(=O)(OC)OC)c3ccccc3NC12</smiles>

4<smiles>[R2]n1c(=O)c(NC(=O)OCc2ccccc2)c(P(=O)(O)OC)c2ccccc21</smiles>

5

\begin{tabular}{|c|c|c|c|c|c|}
\hline entry $^{a}$ & $3\left(\mathrm{R}^{2}\right)$ & time $(\mathrm{h})$ & yield of $4(\%)^{b}$ & ee $(\%)^{c}$ & yield of $5(\%)^{b}$ \\
\hline 1 & 3aa $(\mathrm{Me})$ & 60 & 4aaa (95) & 98 & 5 \\
\hline 2 & 3oa $(\mathrm{H})$ & 144 & 4aoa (81) & 72 & - \\
\hline 3 & $3 p a(A c)$ & 144 & 4apa (50) & 47 & - \\
\hline 4 & 3qa (Bn) & 36 & 4aqa (62) & 57 & 33 \\
\hline 5 & 3ra (MOM) & 42 & 4ara (80) & 40 & 19 \\
\hline
\end{tabular}

${ }^{a}$ All reactions were performed under argon with 2a $(0.1 \mathrm{mmol}), 3(0.11 \mathrm{mmol}), 4 \AA \mathrm{MS}(50 \mathrm{mg}), \mathbf{C P A}(10 \mathrm{~mol} \%)$ and $\mathrm{Ag}_{2} \mathrm{CO}_{3}$ $(5 \mathrm{~mol} \%)$ in $1.0 \mathrm{~mL}$ of $\mathrm{PhMe} / \mathrm{PhEt}(1: 1)$ at $-30^{\circ} \mathrm{C} .{ }^{\mathrm{b}}$ Isolated yield. ${ }^{\mathrm{c}}$ Determined by HPLC analysis. 


\section{General procedure for the asymmetric Mannich reaction}

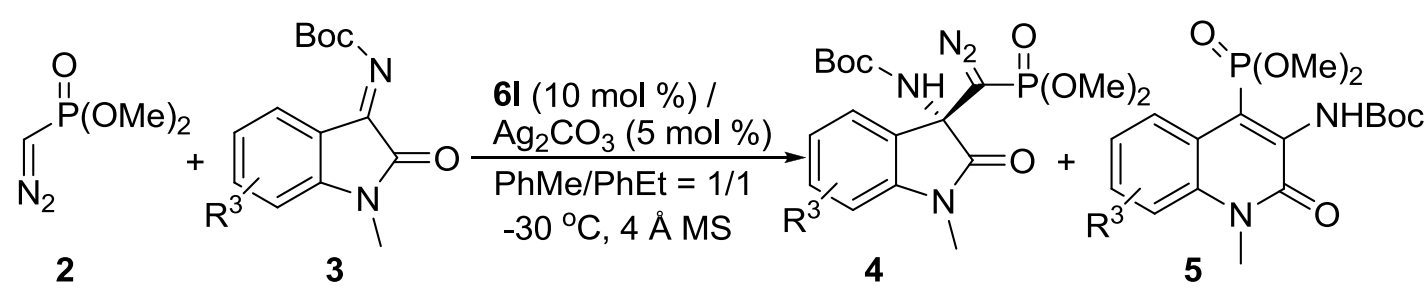

The reaction tube containing powdered $4 \AA$ MS (50 mg) was flame-dried 10 min under vacuum. The atmosphere was replaced by nitrogen. To the dry reaction tube, catalyst $6 \mathrm{I}\left(0.01 \mathrm{mmol}, 0.1\right.$ equiv.), $\mathrm{Ag}_{2} \mathrm{CO}_{3}(0.005 \mathrm{mmol}, 0.05$ equiv.), anhydrous $\mathrm{PhMe}(0.5 \mathrm{~mL})$ and anhydrous $\mathrm{PhEt}(0.5 \mathrm{~mL})$ were added. The resulting solution was stirred for $2.0 \mathrm{~h}$ at the ambient temperature in dark. After the corresponding isatin-derived ketimines $3(0.11 \mathrm{mmol}, 1.1$ equiv $)$ was added and the mixture was cooled to $-30^{\circ} \mathrm{C}$. the corresponding diazomethylphosphonate $2(0.1 \mathrm{mmol}, 1.0$ equiv $)$ was added. The reaction mixture was stirred at $-30^{\circ} \mathrm{C}$ until the reaction furnished, the solution was directly purified by column chromatography on silica gel (PE/EA $=3 / 1$ to $1 / 1$ ) to give the corresponding 3 -substituted 3-aminooxindole 4.

\section{Characterization of the Mannich products 4}<smiles>CN1C(=O)[C@](NC(=O)O)(C(=O)OC(C)(C)C)c2ccccc21</smiles>

7.27 (s, 1H), 7.06 (t, $J=7.5 \mathrm{~Hz}, 1 \mathrm{H}), 7.00$ (d, J=7.8 Hz, 1H), 3.64 (d, J=11.9 Hz, 3H), 3.39 (d, J = 11.9 Hz, 3H), $3.23(\mathrm{~s}, 3 \mathrm{H}), 1.22$ (s, 9H). ${ }^{13} \mathrm{C}$ NMR $\left(151 \mathrm{MHz}\right.$, Acetone-d $\left.\mathrm{d}^{6}\right) \delta 174.9\left({ }^{3} \mathrm{~J}\left({ }^{13} \mathrm{C},{ }^{31} \mathrm{P}\right)=3.4 \mathrm{~Hz}\right), 154.6,144.9,130.5,128.1,126.0,122.8,109.0,80.3,58.6$ $\left({ }^{2} J\left({ }^{13} \mathrm{C},{ }^{31} \mathrm{P}\right)=10.3 \mathrm{~Hz}\right), 53.3\left({ }^{2} \mathrm{~J}\left({ }^{13} \mathrm{C},{ }^{31} \mathrm{P}\right)=5.3 \mathrm{~Hz}\right), 53.2\left({ }^{2} \mathrm{~J}\left({ }^{13} \mathrm{C},{ }^{31} \mathrm{P}\right)=5.3 \mathrm{~Hz}\right), 47.6\left({ }^{1} \mathrm{~J}\left({ }^{13} \mathrm{C},{ }^{31} \mathrm{P}\right)=231.2 \mathrm{~Hz}\right), 28.3,26.8 . \mathrm{HRMS}$ (ESI-TOF) calcd. for $\mathrm{C}_{17} \mathrm{H}_{23} \mathrm{~N}_{4} \mathrm{O}_{6} \mathrm{P}[\mathrm{M}+\mathrm{Na}]^{+}:$433.1253, found: 433.1253 .<smiles>C[R](C)(=O)N[C@]1(C(=O)O[Na])C(=O)Nc2ccccc21</smiles>

(S)-tert-butyl (3-(diazo(dimethoxyphosphoryl)methyl)-2-oxoindolin-3-yl)carbamate (4aoa): Reaction of $2 \mathrm{a}(15.0 \mathrm{mg}, 0.1 \mathrm{mmol}), 30 \mathrm{a}(27.0 \mathrm{mg}, 0.11 \mathrm{mmol})$ according to the general procedure for $144 \mathrm{~h}$ gave 4 aoo as a yellow solid $(32.0 \mathrm{mg}, 81 \%$ yield, $72 \%$ ee $) . \mathrm{mp}: 141-142{ }^{\circ} \mathrm{C},[\mathrm{a}]_{0}^{20}=-27.5^{\circ}(\mathrm{c}=$ 1.0, $\mathrm{CH}_{2} \mathrm{Cl}_{2}$ ); HPLC condition: chiralpak IA, $254 \mathrm{~nm}, 0.5 \mathrm{~mL} / \mathrm{min}$, hexane $/ \mathrm{i}-\mathrm{PrOH}=70 / 30, t_{\text {major }}=$ $15.536 \mathrm{~min}, t_{\text {minor }}=13.513 \mathrm{~min} ;{ }^{1} \mathrm{H}$ NMR $\left(600 \mathrm{MHz}, \mathrm{CD}_{3} \mathrm{OD}\right) \delta 7.35(\mathrm{~d}, J=7.5 \mathrm{~Hz}, 1 \mathrm{H}), 7.31$ (t, $J=7.6$ $\mathrm{Hz}, 1 \mathrm{H}), 7.05$ (t, $J=7.5 \mathrm{~Hz}, 1 \mathrm{H}), 6.92(\mathrm{~d}, J=7.7 \mathrm{~Hz}, 1 \mathrm{H}), 3.67$ (d, $J=11.9 \mathrm{~Hz}, 3 \mathrm{H}), 3.42(\mathrm{~d}, J=11.9$ $\mathrm{Hz}, 3 \mathrm{H}), 1.27$ (dd, $J=15.3,8.1 \mathrm{~Hz}, 9 \mathrm{H}) \mathrm{ppm} .{ }^{13} \mathrm{C} \mathrm{NMR}\left(151 \mathrm{MHz}, \mathrm{CD}_{3} \mathrm{OD}\right) \delta 176.7\left({ }^{3} \mathrm{~J}\left({ }^{13} \mathrm{C},{ }^{31} \mathrm{P}\right)=3.2 \mathrm{~Hz}\right), 154.6,141.8,129.6,127.1$, 125.1, 121.7, 109.7, $57.7\left({ }^{2} J\left({ }^{13} \mathrm{C},{ }^{31} \mathrm{P}\right)=10.4 \mathrm{~Hz}\right), 52.4\left({ }^{2} \mathrm{~J}\left({ }^{13} \mathrm{C},{ }^{31} \mathrm{P}\right)=5.7 \mathrm{~Hz}\right), 52.4\left({ }^{2} \mathrm{~J}\left({ }^{13} \mathrm{C},{ }^{31} \mathrm{P}\right)=5.7 \mathrm{~Hz}\right), 45.6,27.0 \mathrm{ppm} . \mathrm{HRMS}$ (ESI-TOF) calcd. for $\mathrm{C}_{16} \mathrm{H}_{21} \mathrm{~N}_{4} \mathrm{O}_{6} \mathrm{P}[\mathrm{M}+\mathrm{Na}]^{+}:$419.1096, found: 419.1095 . 
<smiles>COC(=O)NC1(C(N)(N)C(=O)OCc2ccccc2)C(=O)N(C(C)(C)C)c2ccccc21</smiles>
ppm. ${ }^{13} \mathrm{C}$ NMR $\left(151 \mathrm{MHz}, \mathrm{CD}_{3} \mathrm{OD}\right) \delta 174.9\left({ }^{3} \mathrm{~J}\left({ }^{13} \mathrm{C},{ }^{31} \mathrm{P}\right)=2.9 \mathrm{~Hz}\right), 170.8,140.2,129.9,126.1,124.7,124.5,115.8,80.7,57.9\left({ }^{2} \mathrm{~J}\left({ }^{13} \mathrm{C}\right.\right.$, $\left.\left.{ }^{31} \mathrm{P}\right)=11.6 \mathrm{~Hz}\right), 52.6\left({ }^{2} J\left({ }^{13} \mathrm{C},{ }^{31} \mathrm{P}\right)=6.2 \mathrm{~Hz}\right), 52.6\left({ }^{2} J\left({ }^{13} \mathrm{C},{ }^{31} \mathrm{P}\right)=5.8 \mathrm{~Hz}\right), 46.1,27.0,25.0 \mathrm{ppm}$. HRMS (ESI-TOF) calcd. for $\mathrm{C}_{18} \mathrm{H}_{23} \mathrm{~N}_{4} \mathrm{O}_{7} \mathrm{P}[\mathrm{M}+\mathrm{Na}]^{+}:$: 461.1202, found: 461.1198 .

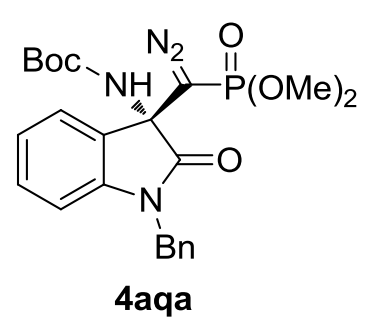

(S)-tert-butyl (1-benzyl-3-(diazo(dimethoxyphosphoryl)methyl)-2-oxoindolin-3-yl)carbamate (4aqa):

Reaction of $2 \mathrm{a}(15.0 \mathrm{mg}, 0.1 \mathrm{mmol}), 3 \mathrm{qa}(37.0 \mathrm{mg}, 0.11 \mathrm{mmol})$ according to the general procedure for $36 \mathrm{~h}$ gave 4aqa as a yellow solid $\left(30.0 \mathrm{mg}, 62 \%\right.$ yield, $57 \%$ ee) $\mathrm{mp}: 71-72{ }^{\circ} \mathrm{C}$, $[\mathrm{\alpha}]_{\mathrm{D}}^{20}=+27.3^{\circ}(\mathrm{c}=1.0$, $\mathrm{CH}_{2} \mathrm{Cl}_{2}$ ); HPLC condition: chiralpak $\mathrm{ODH}, 254 \mathrm{~nm}, 0.5 \mathrm{~mL} / \mathrm{min}$, hexane $/ \mathrm{i}-\mathrm{PrOH}=80 / 20, t_{\text {minor }}=11.119$ $\min , t_{\text {major }}=13.292 \mathrm{~min} ;{ }^{1} \mathrm{H}$ NMR $\left(600 \mathrm{MHz}, \mathrm{CD}_{3} \mathrm{OD}\right) \delta 7.48(\mathrm{~d}, J=7.6 \mathrm{~Hz}, 2 \mathrm{H}), 7.39(\mathrm{~d}, J=7.5 \mathrm{~Hz}, 1 \mathrm{H})$, 7.35 (t, $J=7.5 \mathrm{~Hz}, 2 \mathrm{H}), 7.28(\mathrm{t}, J=7.1 \mathrm{~Hz}, 2 \mathrm{H}), 7.08(\mathrm{t}, J=7.3 \mathrm{~Hz}, 1 \mathrm{H}), 6.90(\mathrm{~s}, 1 \mathrm{H}), 5.14(\mathrm{~s}, 1 \mathrm{H}), 4.59$ (s, $1 \mathrm{H}), 3.62(\mathrm{~d}, J=11.9 \mathrm{~Hz}, 3 \mathrm{H}), 3.15(\mathrm{~d}, J=10.4 \mathrm{~Hz}, 3 \mathrm{H}), 1.33(\mathrm{~d}, J=23.5 \mathrm{~Hz}, 9 \mathrm{H}) \mathrm{ppm} .{ }^{13} \mathrm{C} \mathrm{NMR}\left(151 \mathrm{MHz}, \mathrm{CD}_{3} \mathrm{OD}\right) \delta 175.0\left({ }^{3} J\right.$ $\left.\left({ }^{13} \mathrm{C},{ }^{31} \mathrm{P}\right)=3.7 \mathrm{~Hz}\right), 154.6,142.6,135.8,129.6,128.4,127.4,126.4,126.2,125.0,122.3,109.1,80.1,57.6\left({ }^{2} \mathrm{~J}\left({ }^{13} \mathrm{C},{ }^{31} \mathrm{P}\right)=11.4 \mathrm{~Hz}\right)$, $52.4\left({ }^{2} \mathrm{~J}\left({ }^{13} \mathrm{C},{ }^{31} \mathrm{P}\right)=5.8 \mathrm{~Hz}\right), 52.3\left({ }^{2} \mathrm{~J}\left({ }^{13} \mathrm{C},{ }^{31} \mathrm{P}\right)=6.0 \mathrm{~Hz}\right) .$, 45.7, 43.6, $27.1 \mathrm{ppm}$. HRMS (ESI-TOF) calcd. for $\mathrm{C}_{23} \mathrm{H}_{27} \mathrm{~N}_{4} \mathrm{O}_{6} \mathrm{P}[\mathrm{M}+\mathrm{Na}]^{+}$: 509.1566, found: 509.1566 .

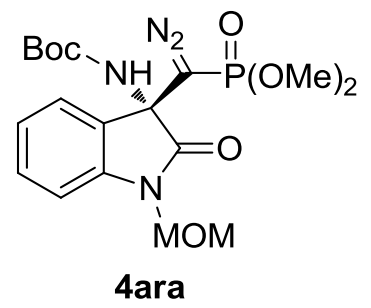

(S)-tert-butyl (3-(diazo(dimethoxyphosphoryl)methyl)-1-(methoxymethyl)-2-oxoindolin-3-yl)carbamate (4ara):

Reaction of $2 \mathrm{a}(15.0 \mathrm{mg}, 0.1 \mathrm{mmol}), 3 \mathrm{ra}(31.9 \mathrm{mg}, 0.11 \mathrm{mmol})$ according to the general procedure for $42 \mathrm{~h}$ gave 4ara as a yellow solid $\left(35.2 \mathrm{mg}, 80 \%\right.$ yield, $40 \%$ ee). mp: $128-129{ }^{\circ} \mathrm{C},[\mathrm{a}]_{\mathrm{D}}^{20}=-30.2^{\circ}(\mathrm{c}=1.0$, $\mathrm{CH}_{2} \mathrm{Cl}_{2}$ ); HPLC condition: chiralpak IA, $254 \mathrm{~nm}, 0.5 \mathrm{~mL} / \mathrm{min}$, hexane $/ \mathrm{i}-\mathrm{PrOH}=70 / 30, t_{\text {major }}=11.631 \mathrm{~min}$, $t_{\text {minor }}=13.589 \mathrm{~min} ;{ }^{1} \mathrm{H} \mathrm{NMR}\left(600 \mathrm{MHz}, \mathrm{CD}_{3} \mathrm{OD}\right) \delta 7.40(\mathrm{dd}, J=16.6,7.7 \mathrm{~Hz}, 2 \mathrm{H}), 7.18-7.10(\mathrm{~m}, 2 \mathrm{H})$, $5.18(\mathrm{dd}, J=72.5,10.9 \mathrm{~Hz}, 2 \mathrm{H}), 3.67(\mathrm{~d}, J=11.9 \mathrm{~Hz}, 3 \mathrm{H}), 3.43(\mathrm{~s}, 3 \mathrm{H}), 3.38(\mathrm{~d}, J=11.8 \mathrm{~Hz}, 3 \mathrm{H}), 1.30$ (s, 9H) ppm. ${ }^{13} \mathrm{C}$ NMR $\left(151 \mathrm{MHz}, \mathrm{CD}_{3} \mathrm{OD}\right) \delta 175.4\left({ }^{3} \mathrm{~J}\left({ }^{13} \mathrm{C},{ }^{31} \mathrm{P}\right)=3.6 \mathrm{~Hz}\right), 154.6,142.1,129.7,126.0,124.9,122.6,109.4,80.2,71.6$, $57.7\left({ }^{2} J\left({ }^{13} \mathrm{C},{ }^{31} \mathrm{P}\right)=10.9 \mathrm{~Hz}\right), 55.6,52.5\left({ }^{2} \mathrm{~J}\left({ }^{13} \mathrm{C},{ }^{31} \mathrm{P}\right)=2.7 \mathrm{~Hz}\right), 52.4\left({ }^{2} J\left({ }^{13} \mathrm{C},{ }^{31} \mathrm{P}\right)=2.9 \mathrm{~Hz}\right), 45.8,27.0$ ppm. HRMS (ESI-TOF) calcd. for $\mathrm{C}_{18} \mathrm{H}_{25} \mathrm{~N}_{4} \mathrm{O} 7 \mathrm{P}[\mathrm{M}+\mathrm{Na}]^{+}:$463.1359, found: 463.1360 .<smiles>CC(=O)N[C@]1(C(=O)[P+](C)=O)C(=O)N(C)c2ccccc21</smiles>

4aab

\section{(S)-ethyl (3-(diazo(dimethoxyphosphoryl)methyl)-1-methyl-2-oxoindolin-3-yl)carbamate (4aab):}

Reaction of $2 \mathbf{a}(15.0 \mathrm{mg}, 0.1 \mathrm{mmol}), 3 \mathbf{a b}(28.9 \mathrm{mg}, 0.11 \mathrm{mmol})$ according to the general procedure for $36 \mathrm{~h}$ gave $4 \mathrm{aab}$ as a yellow solid $(28.0 \mathrm{mg}, 73 \%$ yield, $99 \%$ ee $) . \mathrm{mp}: 182-183^{\circ} \mathrm{C},[\mathrm{\alpha}]_{\mathrm{D}}^{20}=+105.7^{\circ}(\mathrm{c}=$ 1.0, $\mathrm{CH}_{2} \mathrm{Cl}_{2}$ ); HPLC condition: chiralpak IA, $254 \mathrm{~nm}, 0.5 \mathrm{~mL} / \mathrm{min}$, hexane/ $\mathrm{i}-\mathrm{PrOH}=90 / 10, t_{\text {major }}=34.300$ $\min , t_{\text {minor }}=45.250 \mathrm{~min} ;{ }^{1} \mathrm{H} \mathrm{NMR}\left(600 \mathrm{MHz}, \mathrm{CD}_{3} \mathrm{OD}\right) \delta 7.40(\mathrm{t}, J=7.3 \mathrm{~Hz}, 2 \mathrm{H}), 7.11(\mathrm{t}, J=7.6 \mathrm{~Hz}, 1 \mathrm{H})$, $7.03(\mathrm{~d}, J=7.9 \mathrm{~Hz}, 1 \mathrm{H}), 3.95(\mathrm{~d}, J=6.7 \mathrm{~Hz}, 2 \mathrm{H}), 3.66$ (d, $J=11.9 \mathrm{~Hz}, 3 \mathrm{H}), 3.40(\mathrm{~d}, J=11.9 \mathrm{~Hz}, 3 \mathrm{H})$, $3.28(\mathrm{~s}, 3 \mathrm{H}), 1.16(\mathrm{~s}, 3 \mathrm{H}) \mathrm{ppm} .{ }^{13} \mathrm{C}$ NMR $\left(151 \mathrm{MHz}, \mathrm{CD}_{3} \mathrm{OD}\right) \delta 174.9\left({ }^{3} \mathrm{~J}\left({ }^{13} \mathrm{C},{ }^{31} \mathrm{P}\right)=3.3 \mathrm{~Hz}\right), 155.5,143.7,129.9,126.1,124.9,122.3$, 108.3, 60.9, $57.4\left({ }^{2} \mathrm{~J}\left({ }^{13} \mathrm{C},{ }^{31} \mathrm{P}\right)=11.5 \mathrm{~Hz}\right), 52.4\left({ }^{2} \mathrm{~J}\left({ }^{13} \mathrm{C},{ }^{31} \mathrm{P}\right)=5.7 \mathrm{~Hz}\right), 52.4\left({ }^{2} \mathrm{~J}\left({ }^{13} \mathrm{C},{ }^{31} \mathrm{P}\right)=5.8 \mathrm{~Hz}\right), 45.6,25.5,13.2 \mathrm{ppm} . \mathrm{HRMS}$ (ESI-TOF) calcd. for $\mathrm{C}_{15} \mathrm{H}_{19} \mathrm{~N}_{4} \mathrm{O}_{6} \mathrm{P}[\mathrm{M}+\mathrm{Na}]^{+}:$405.0940, found: 405.0939 . 


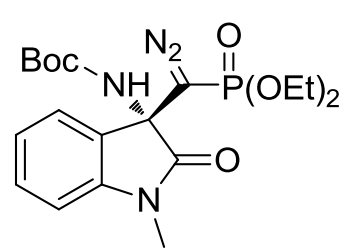

4baa

(S)-tert-butyl (3-(diazo(diethoxyphosphoryl)methyl)-1-methyl-2-oxoindolin-3-yl)carbamate (4baa):

Reaction of $\mathbf{2 b}(17.8 \mathrm{mg}, 0.1 \mathrm{mmol})$, 3aa $(28.6 \mathrm{mg}, 0.11 \mathrm{mmol})$ according to the general procedure for $12 \mathrm{~h}$ gave 4 aab as a yellow solid $(28.0 \mathrm{mg}, 64 \%$ yield, $99 \%$ ee $)$. mp: $134-135{ }^{\circ} \mathrm{C}$, $[\mathrm{a}]_{\circ}^{20}=+91.3^{\circ}(\mathrm{c}=1.0$, $\mathrm{CH}_{2} \mathrm{Cl}_{2}$ ); HPLC condition: chiralpak IA, $254 \mathrm{~nm}, 0.5 \mathrm{~mL} / \mathrm{min}$, hexane $/ \mathrm{i}-\mathrm{PrOH}=70 / 30, t_{\text {major }}=10.600 \mathrm{~min}$, $t_{\text {minor }}=13.818 \mathrm{~min} ;{ }^{1} \mathrm{H}$ NMR $\left(600 \mathrm{MHz}, \mathrm{CD}_{3} \mathrm{OD}\right) \delta 7.41(\mathrm{dd}, J=12.5,7.6 \mathrm{~Hz}, 2 \mathrm{H}), 7.11(\mathrm{t}, J=7.4 \mathrm{~Hz}, 1 \mathrm{H})$, $7.02(\mathrm{~d}, J=7.7 \mathrm{~Hz}, 1 \mathrm{H}), 4.07-3.92(\mathrm{~m}, 2 \mathrm{H}), 3.85-3.70(\mathrm{~m}, 2 \mathrm{H}), 3.27(\mathrm{~s}, 3 \mathrm{H}), 1.28(\mathrm{t}, J=7.0 \mathrm{~Hz}, 12 \mathrm{H})$, $1.06(\mathrm{t}, J=7.0 \mathrm{~Hz}, 3 \mathrm{H}) \mathrm{ppm} .{ }^{13} \mathrm{C}$ NMR $\left(151 \mathrm{MHz}, \mathrm{CD}_{3} \mathrm{OD}\right) \delta 175.1\left({ }^{3} \mathrm{~J}\left({ }^{13} \mathrm{C},{ }^{31} \mathrm{P}\right)=3.5 \mathrm{~Hz}\right), 154.5,143.7,129.8,126.6,125.1,122.2$, 108.3, 80.2, $63.0\left({ }^{2} \mathrm{~J}\left({ }^{13} \mathrm{C},{ }^{31} \mathrm{P}\right)=5.9 \mathrm{~Hz}\right), 62.8\left({ }^{2} \mathrm{~J}\left({ }^{13} \mathrm{C},{ }^{31} \mathrm{P}\right)=5.9 \mathrm{~Hz}\right), 57.5\left({ }^{2} \mathrm{~J}\left({ }^{13} \mathrm{C},{ }^{31} \mathrm{P}\right)=11.0 \mathrm{~Hz}\right) ., 46.3,27.0,25.5,15.0,14.9,14.8$, 14.7 ppm. HRMS (ESI-TOF) calcd. for $\mathrm{C}_{19} \mathrm{H}_{27} \mathrm{~N}_{4} \mathrm{O}_{6} \mathrm{P}[\mathrm{M}+\mathrm{Na}]^{+}: 461.1566$, found: 461.1584 .

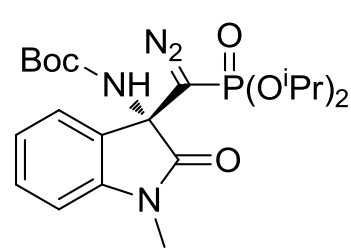

4caa

(S)-tert-butyl (3-(diazo(diisopropoxyphosphoryl)methyl)-1-methyl-2-oxoindolin-3-yl)carbamate (4caa):

Reaction of $2 \mathrm{c}(20.6 \mathrm{mg}, 0.1 \mathrm{mmol})$, 3aa $(28.6 \mathrm{mg}, 0.11 \mathrm{mmol})$ according to the general procedure for $36 \mathrm{~h}$ gave 4 caa as a yellow solid $\left(20.0 \mathrm{mg}, 43 \%\right.$ yield, $96 \%$ ee). $\mathrm{mp}: 121-122{ }^{\circ} \mathrm{C},[\mathrm{a}]_{0}^{20}=+106.7^{\circ}(\mathrm{c}=1.0$, $\mathrm{CH}_{2} \mathrm{Cl}_{2}$ ); HPLC condition: chiralpak IA, $254 \mathrm{~nm}, 0.5 \mathrm{~mL} / \mathrm{min}$, hexane $/ \mathrm{i}-\mathrm{PrOH}=70 / 30, t_{\text {major }}=8.293 \mathrm{~min}$, $t_{\text {minor }}=10.552 \mathrm{~min} ;{ }^{1} \mathrm{H}$ NMR $\left(600 \mathrm{MHz}, \mathrm{CD}_{3} \mathrm{OD}\right) \delta 7.44(\mathrm{~d}, J=7.4 \mathrm{~Hz}, 1 \mathrm{H}), 7.38(\mathrm{t}, J=7.6 \mathrm{~Hz}, 1 \mathrm{H}), 7.08$ (t, $J=7.5 \mathrm{~Hz}, 1 \mathrm{H}), 6.99$ (d, $J=7.8 \mathrm{~Hz}, 1 \mathrm{H}), 4.54$ (tt, $J=12.4,6.2 \mathrm{~Hz}, 1 \mathrm{H}), 4.42(\mathrm{dq}, J=12.6,6.2 \mathrm{~Hz}$, $1 \mathrm{H}), 3.24(\mathrm{~s}, 3 \mathrm{H}), 1.27(\mathrm{dd}, J=18.5,6.2 \mathrm{~Hz}, 12 \mathrm{H}), 1.06(\mathrm{~d}, J=6.2 \mathrm{~Hz}, 6 \mathrm{H}) \mathrm{ppm} .{ }^{13} \mathrm{C} \mathrm{NMR}\left(151 \mathrm{MHz}, \mathrm{CD}_{3} \mathrm{OD}\right) \delta 175.0\left({ }^{3} J\left({ }^{13} \mathrm{C},{ }^{31} \mathrm{P}\right)=\right.$ 3.9 Hz), 154.5, 143.8, 129.7, 126.8, 125.4, 122.2, 108.3, 80.1, $72.6\left({ }^{2} J\left({ }^{13} \mathrm{C},{ }^{31} \mathrm{P}\right)=6.3 \mathrm{~Hz}\right), 72.5\left({ }^{2} \mathrm{~J}\left({ }^{13} \mathrm{C},{ }^{31} \mathrm{P}\right)=6.3 \mathrm{~Hz}\right), 57.6\left({ }^{2} \mathrm{~J}\left({ }^{13} \mathrm{C}\right.\right.$ $\left.\left.{ }^{31} \mathrm{P}\right)=10.7 \mathrm{~Hz}\right), 48.8,27.0,25.5,22.7\left({ }^{3} \mathrm{~J}\left({ }^{13} \mathrm{C},{ }^{31} \mathrm{P}\right)=4.1 \mathrm{~Hz}\right), 22.6\left({ }^{3} \mathrm{~J}\left({ }^{13} \mathrm{C},{ }^{31} \mathrm{P}\right)=4.7 \mathrm{~Hz}\right), 22.2\left({ }^{3} \mathrm{~J}\left({ }^{13} \mathrm{C},{ }^{31} \mathrm{P}\right)=5.3 \mathrm{~Hz}\right) \mathrm{ppm} . \mathrm{HRMS}$ (ESI-TOF) calcd. for $\mathrm{C}_{21} \mathrm{H}_{31} \mathrm{~N}_{4} \mathrm{O}_{6} \mathrm{P}[\mathrm{M}+\mathrm{Na}]^{+}:$489.1879, found: 489.1886 .<smiles>CN1C(=O)[C@](NC(C)(C)C)(C(=O)[Po](C)(C)C)c2cc(F)ccc21</smiles>

4aba

(S)-tert-butyl (3-(diazo(dimethoxyphosphoryl)methyl)-5-fluoro-1-methyl-2-oxoindolin-3-yl)carbamate (4aba):

Reaction of $2 \mathbf{a}(15.0 \mathrm{mg}, 0.1 \mathrm{mmol}), 3 \mathbf{b a}(30.6 \mathrm{mg}, 0.11 \mathrm{mmol})$ according to the general procedure for $144 \mathrm{~h}$ gave 4 aba as a yellow solid $\left(39.8 \mathrm{mg}, 93 \%\right.$ yield, $98 \%$ ee) $. \mathrm{mp}: 134-135^{\circ} \mathrm{C},[\alpha]_{0}^{20}=+87.8^{\circ}$ ( $\mathrm{c}=1.0, \mathrm{CH}_{2} \mathrm{Cl}_{2}$ ); HPLC condition: chiralpak IA, $254 \mathrm{~nm}, 0.5 \mathrm{~mL} / \mathrm{min}$, hexane $/ \mathrm{i}-\mathrm{PrOH}=90 / 10, t_{\text {major }}$ $=35.710 \mathrm{~min}, t_{\text {minor }}=43.680 \mathrm{~min} ;{ }^{1} \mathrm{H}$ NMR $\left(600 \mathrm{MHz}, \mathrm{CD}_{3} \mathrm{OD}\right) \delta 7.17(\mathrm{dd}, J=22.2,7.9 \mathrm{~Hz}, 2 \mathrm{H})$, $7.07-6.95(\mathrm{~m}, 1 \mathrm{H}), 3.69(\mathrm{~d}, J=11.9 \mathrm{~Hz}, 3 \mathrm{H}), 3.43(\mathrm{~d}, J=11.9 \mathrm{~Hz}, 3 \mathrm{H}), 3.27(\mathrm{~s}, 3 \mathrm{H}), 1.31(\mathrm{~s}, 9 \mathrm{H})$ ppm. ${ }^{13} \mathrm{C}$ NMR $\left(151 \mathrm{MHz}, \mathrm{CD}_{3} \mathrm{OD}\right) \delta 174.8\left({ }^{3} \mathrm{~J}\left({ }^{13} \mathrm{C},{ }^{31} \mathrm{P}\right)=3.4 \mathrm{~Hz}\right), 159.9,158.3,154.5,139.7,128.1,115.9\left({ }^{2} \mathrm{~J}\left({ }^{13} \mathrm{C},{ }^{19} \mathrm{~F}\right)=23.8 \mathrm{~Hz}\right)$, $112.8\left({ }^{1} J\left({ }^{13} \mathrm{C},{ }^{19} \mathrm{~F}\right)=26.0 \mathrm{~Hz}\right), 109.2\left({ }^{2} \mathrm{~J}\left({ }^{13} \mathrm{C},{ }^{19} \mathrm{~F}\right)=8.0 \mathrm{~Hz}\right), 80.4,57.5\left({ }^{2} \mathrm{~J}\left({ }^{13} \mathrm{C},{ }^{31} \mathrm{P}\right)=11.8 \mathrm{~Hz}\right), 52.5\left({ }^{2} J\left({ }^{13} \mathrm{C},{ }^{31} \mathrm{P}\right)=5.8 \mathrm{~Hz}\right), 52.4\left({ }^{2} J\right.$ $\left.\left({ }^{13} \mathrm{C},{ }^{31} \mathrm{P}\right)=5.8 \mathrm{~Hz}\right), 45.5,27.0,25.7$ ppm. HRMS (ESI-TOF) calcd. for $\mathrm{C}_{17} \mathrm{H}_{22} \mathrm{FN}_{4} \mathrm{O}_{6} \mathrm{P}[\mathrm{M}+\mathrm{Na}]^{+}: 451.1159$, found: 451.1158.

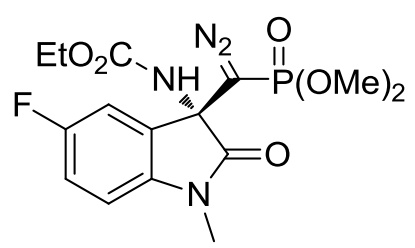

4abb
(S)-ethyl (3-(diazo(dimethoxyphosphoryl)methyl)-5-fluoro-1-methyl-2-oxoindolin-3-yl)carbamate (4abb):

Reaction of $2 \mathrm{a}(15.0 \mathrm{mg}, 0.1 \mathrm{mmol}), 3 \mathbf{b b}(27.5 \mathrm{mg}, 0.11 \mathrm{mmol})$ according to the general procedure for $144 \mathrm{~h}$ gave 4 aba as a yellow solid $\left(34.0 \mathrm{mg}, 85 \%\right.$ yield, $99 \%$ ee). $\mathrm{mp}: 144-145{ }^{\circ} \mathrm{C}$, $[\alpha]_{0}^{20}=$ $+100.8^{\circ}\left(\mathrm{c}=1.0, \mathrm{CH}_{2} \mathrm{Cl}_{2}\right.$ ); HPLC con dition: chiralpak IA, $254 \mathrm{~nm}, 0.5 \mathrm{~mL} / \mathrm{min}$, hexane $/ \mathrm{i}-\mathrm{PrOH}=$ 90/10, $t_{\text {major }}=27.160 \mathrm{~min}, t_{\text {minor }}=33.250 \mathrm{~min} ;{ }^{1} \mathrm{H} \mathrm{NMR}\left(600 \mathrm{MHz}, \mathrm{CD}_{3} \mathrm{OD}\right) \delta 7.19(\mathrm{dd}, J=8.0,2.5$ $\mathrm{Hz}, 1 \mathrm{H}$ ), 7.15 (td, $J=9.0,2.6 \mathrm{~Hz}, 1 \mathrm{H}), 7.02(\mathrm{dd}, J=8.5,4.0 \mathrm{~Hz}, 1 \mathrm{H}), 3.98(\mathrm{~d}, J=6.5 \mathrm{~Hz}, 2 \mathrm{H}), 3.70$ $(\mathrm{d}, J=11.9 \mathrm{~Hz}, 3 \mathrm{H}), 3.44(\mathrm{~d}, J=11.9 \mathrm{~Hz}, 3 \mathrm{H}), 3.27(\mathrm{~s}, 3 \mathrm{H}), 1.17(\mathrm{~s}, 3 \mathrm{H}) \mathrm{ppm} .{ }^{13} \mathrm{C}$ NMR $\left(151 \mathrm{MHz}, \mathrm{CD}_{3} \mathrm{OD}\right) \delta 174.6\left({ }^{3} J\left({ }^{13} \mathrm{C},{ }^{31} \mathrm{P}\right)=\right.$ 3.3 Hz), 159.9, 158.3, 155.6, 139.8, 127.8, 127.7, $116.0\left({ }^{2} \mathrm{~J}\left({ }^{13} \mathrm{C},{ }^{19} \mathrm{~F}\right)=23.8 \mathrm{~Hz}\right), 113.0\left({ }^{1} \mathrm{~J}\left({ }^{13} \mathrm{C},{ }^{19} \mathrm{~F}\right)=26.1 \mathrm{~Hz}\right), 109.2\left({ }^{2} \mathrm{~J}\left({ }^{13} \mathrm{C},{ }^{19} \mathrm{~F}\right)=\right.$ $8.0 \mathrm{~Hz}), 61.1,57.5\left({ }^{2} \mathrm{~J}\left({ }^{13} \mathrm{C},{ }^{31} \mathrm{P}\right)=11.0 \mathrm{~Hz}\right), 52.5\left({ }^{2} \mathrm{~J}\left({ }^{13} \mathrm{C},{ }^{31} \mathrm{P}\right)=5.8 \mathrm{~Hz}\right), 52.4\left({ }^{2} \mathrm{~J}\left({ }^{13} \mathrm{C},{ }^{31} \mathrm{P}\right)=5.8 \mathrm{~Hz}\right), 45.5,25.7,13.2 \mathrm{ppm} . \mathrm{HRMS}$ (ESI-TOF) calcd. for $\mathrm{C}_{15} \mathrm{H}_{18} \mathrm{FN}_{4} \mathrm{O}_{6} \mathrm{P}[\mathrm{M}+\mathrm{Na}]^{+}:$423.0846, found: 423.0884 . 


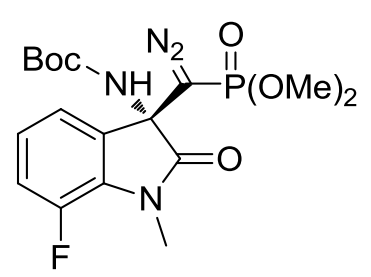

4aca

Reaction of $2 \mathrm{a}(15.0 \mathrm{mg}, 0.1 \mathrm{mmol}), 3 \mathrm{ca}(30.6 \mathrm{mg}, 0.11 \mathrm{mmol})$ according to the general procedure for $12 \mathrm{~h}$ gave 4 aba as a yellow solid $(27.0 \mathrm{mg}, 63 \%$ yield, $98 \%$ ee $)$. mp: $140-141^{\circ} \mathrm{C}$, $[\alpha]_{0}^{20}=+156.7^{\circ}(\mathrm{c}=$ 1.0, $\mathrm{CH}_{2} \mathrm{Cl}_{2}$ ); HPLC condition: chiralpak IA, $254 \mathrm{~nm}, 0.5 \mathrm{~mL} / \mathrm{min}$, hexane/ $\mathrm{i}-\mathrm{PrOH}=80 / 20, t_{\text {major }}=17.889$ $\min , t_{\text {minor }}=26.117 \mathrm{~min} ;{ }^{1} \mathrm{H}$ NMR $\left(600 \mathrm{MHz}, \mathrm{CD}_{3} \mathrm{OD}\right) \delta 7.26-7.14(\mathrm{~m}, 2 \mathrm{H}), 7.09(\mathrm{dt}, \mathrm{J}=12.1,6.2 \mathrm{~Hz}$, $1 \mathrm{H}), 3.67(\mathrm{~d}, J=11.9 \mathrm{~Hz}, 3 \mathrm{H}), 3.50-3.43(\mathrm{~m}, 6 \mathrm{H}), 1.28(\mathrm{~d}, J=27.0 \mathrm{~Hz}, 9 \mathrm{H}) \mathrm{ppm} .{ }^{13} \mathrm{C} \mathrm{NMR}(151 \mathrm{MHz}$, $\left.\mathrm{CD}_{3} \mathrm{OD}\right) \delta 174.7\left({ }^{3} \mathrm{~J}\left({ }^{13} \mathrm{C},{ }^{31} \mathrm{P}\right)=3.3 \mathrm{~Hz}\right), 154.5,148.6,147.0,130.1\left({ }^{2} \mathrm{~J}\left({ }^{13} \mathrm{C},{ }^{19} \mathrm{~F}\right)=8.3 \mathrm{~Hz}\right), 129.5,123.00\left({ }^{2} \mathrm{~J}\left({ }^{13} \mathrm{C},{ }^{19} \mathrm{~F}\right)=6.4 \mathrm{~Hz}\right)$, $120.8\left({ }^{2} J\left({ }^{13} \mathrm{C},{ }^{19} \mathrm{~F}\right)=3.2 \mathrm{~Hz}\right), 117.5\left({ }^{1} J\left({ }^{13} \mathrm{C},{ }^{19} \mathrm{~F}\right)=19.6 \mathrm{~Hz}\right), 80.4,57.4\left({ }^{2} J\left({ }^{13} \mathrm{C},{ }^{31} \mathrm{P}\right)=6.1 \mathrm{~Hz}\right), 52.5\left({ }^{2} J\left({ }^{13} \mathrm{C},{ }^{31} \mathrm{P}\right)=2.6 \mathrm{~Hz}\right), 52.5\left({ }^{2} J\right.$ $\left.\left({ }^{13} \mathrm{C},{ }^{31} \mathrm{P}\right)=2.7 \mathrm{~Hz}\right), 45.7,28.1,28.1,27.0$ ppm. HRMS (ESI-TOF) calcd. for $\mathrm{C}_{17} \mathrm{H}_{22} \mathrm{FN}_{4} \mathrm{O} 6 \mathrm{P}[\mathrm{M}+\mathrm{Na}]^{+}: 451.1159$, found: 451.1157.<smiles>CCOC(=O)N[C@]1(C(N)=O)C(=O)N(C)c2c(F)cccc21</smiles>

$6 \mathrm{H}), 1.17$ (s, 3H) ppm. ${ }^{13} \mathrm{C}$ NMR $\left(151 \mathrm{MHz}, \mathrm{CD}_{3} \mathrm{OD}\right) \delta 174.6\left({ }^{3} \mathrm{~J}\left({ }^{13} \mathrm{C},{ }^{31} \mathrm{P}\right)=3.3 \mathrm{~Hz}\right), 155.5,148.6,146.9,130.2\left({ }^{2} \mathrm{~J}\left({ }^{13} \mathrm{C},{ }^{19} \mathrm{~F}\right)=8.9\right.$ $\mathrm{Hz}), 129.1,123.1\left({ }^{2} \mathrm{~J}\left({ }^{13} \mathrm{C},{ }^{19} \mathrm{~F}\right)=6.5 \mathrm{~Hz}\right), 121.0\left({ }^{2} \mathrm{~J}\left({ }^{13} \mathrm{C},{ }^{19} \mathrm{~F}\right)=3.2 \mathrm{~Hz}\right), 117.6\left({ }^{1} \mathrm{~J}\left({ }^{13} \mathrm{C},{ }^{19} \mathrm{~F}=19.5 \mathrm{~Hz}\right), 61.1,57.4\left({ }^{2} \mathrm{~J}\left({ }^{13} \mathrm{C},{ }^{31} \mathrm{P}\right)=11.5\right.\right.$ $\mathrm{Hz})$, 53.4, $52.5\left({ }^{2} \mathrm{~J}\left({ }^{13} \mathrm{C},{ }^{31} \mathrm{P}\right)=0.7 \mathrm{~Hz}\right), 52.5\left({ }^{2} \mathrm{~J}\left({ }^{13} \mathrm{C},{ }^{31} \mathrm{P}\right)=0.7 \mathrm{~Hz}\right) ., 45.6,28.2,28.1,13.2 \mathrm{ppm}$. HRMS (ESI-TOF) calcd. for $\mathrm{C}_{15} \mathrm{H}_{18} \mathrm{FN}_{4} \mathrm{O}_{6} \mathrm{P}[\mathrm{M}+\mathrm{Na}]^{+}:$423.0846, found: 423.0851 .<smiles>CO[P+]([O-])([O-])[C@]1(NC(=O)[O-])C(=O)N(C)c2ccc(Cl)cc21</smiles>

4ada

(S)-ethyl (3-(diazo(dimethoxyphosphoryl)methyl)-7-fluoro-1-methyl-2-oxoindolin-3-yl)carbamate (4acb):

Reaction of $2 \mathbf{a}(15.0 \mathrm{mg}, 0.1 \mathrm{mmol}), 3 \mathrm{cb}(27.5 \mathrm{mg}, 0.11 \mathrm{mmol})$ according to the general procedure for $84 \mathrm{~h}$ gave 4 aca as a yellow solid (33.2 mg, 83\% yield, 99\% ee). mp: $120-121^{\circ} \mathrm{C},[\alpha]_{0}^{20}=+135.0^{\circ}$ $\left(\mathrm{c}=1.0, \mathrm{CH}_{2} \mathrm{Cl}_{2}\right.$ ); HPLC condition: chiralpak IA, $254 \mathrm{~nm}, 0.5 \mathrm{~mL} / \mathrm{min}$, hexane $/ \mathrm{i}-\mathrm{PrOH}=80 / 20$, $t_{\text {major }}=$ $12.846 \mathrm{~min}, t_{\text {minor }}=15.300 \mathrm{~min} ;{ }^{1} \mathrm{H}$ NMR $\left(600 \mathrm{MHz}, \mathrm{CD}_{3} \mathrm{OD}\right) \delta 7.20(\mathrm{ddd}, J=20.2,9.6,4.7 \mathrm{~Hz}, 2 \mathrm{H})$, 7.09 (td, $J=8.0,4.4 \mathrm{~Hz}, 1 \mathrm{H}), 4.04-3.91(\mathrm{~m}, 2 \mathrm{H}), 3.68(\mathrm{~d}, J=11.9 \mathrm{~Hz}, 3 \mathrm{H}), 3.47$ (dd, $J=7.3,4.6 \mathrm{~Hz}$, (S)-tert-butyl (5-chloro-3-(diazo(dimethoxyphosphoryl)methyl)-1-methyl-2-oxoindolin-3-yl)carbamate (4ada): Reaction of $2 \mathrm{a}(15.0 \mathrm{mg}, 0.1 \mathrm{mmol})$, 3da $(32.4 \mathrm{mg}, 0.11 \mathrm{mmol})$ according to the general procedure for $36 \mathrm{~h}$ gave 4 ada as a yellow solid (37.8 $\mathrm{mg}, 85 \%$ yield, $96 \%$ ee). $\mathrm{mp}: 146-147{ }^{\circ} \mathrm{C}$, [a] ${ }_{D}^{20}=+81.7^{\circ}\left(\mathrm{C}=1.0, \mathrm{CH}_{2} \mathrm{Cl}_{2}\right)$; HPLC condition: chiralpak IA, $254 \mathrm{~nm}, 0.5 \mathrm{~mL} / \mathrm{min}$, hexane $/ \mathrm{i}-\mathrm{PrOH}=$ 90/10, $t_{\text {major }}=31.374 \mathrm{~min}, t_{\text {minor }}=37.226 \mathrm{~min} ;{ }^{1} \mathrm{H}$ NMR $\left(600 \mathrm{MHz}, \mathrm{CD}_{3} \mathrm{OD}\right) \delta 7.40(\mathrm{dd}, J=17.0,5.1$ $\mathrm{Hz}, 2 \mathrm{H}), 7.02(\mathrm{~d}, J=8.3 \mathrm{~Hz}, 1 \mathrm{H}), 3.70(\mathrm{~d}, J=11.9 \mathrm{~Hz}, 3 \mathrm{H}), 3.42(\mathrm{~d}, J=11.9 \mathrm{~Hz}, 3 \mathrm{H}), 3.27(\mathrm{~s}, 3 \mathrm{H})$, $1.32(\mathrm{~s}, 9 \mathrm{H}) \mathrm{ppm} .{ }^{13} \mathrm{C}$ NMR $\left(151 \mathrm{MHz}, \mathrm{CD}_{3} \mathrm{OD}\right) \delta 174.6\left({ }^{3} \mathrm{~J}\left({ }^{13} \mathrm{C},{ }^{31} \mathrm{P}\right)=3.5 \mathrm{~Hz}\right), 154.5,142.4,129.6,128.2,127.6,125.2,109.6,80.4$, $57.2\left({ }^{2} J\left({ }^{13} \mathrm{C},{ }^{31} \mathrm{P}\right)=10.2 \mathrm{~Hz}\right), 52.6\left({ }^{2} J\left({ }^{13} \mathrm{C},{ }^{31} \mathrm{P}\right)=5.9 \mathrm{~Hz}\right), 52.4\left({ }^{2} J\left({ }^{13} \mathrm{C},{ }^{31} \mathrm{P}\right)=5.8 \mathrm{~Hz}\right), 45.5,27.0,25.7 \mathrm{ppm}$. HRMS (ESI-TOF) calcd. for $\mathrm{C}_{17} \mathrm{H}_{22} \mathrm{CIN}_{4} \mathrm{O}_{6} \mathrm{P}[\mathrm{M}+\mathrm{Na}]^{+}:$467.0863, found: 467.0860 .<smiles>CCOC(=O)N[C@@]1(C(N)=O)C(=O)N(C)c2ccc(Cl)cc21</smiles>

$4 a d b$

(S)-ethyl (5-chloro-3-(diazo(dimethoxyphosphoryl)methyl)-1-methyl-2-oxoindolin-3-yl)carbamate (4adb): Reaction of $2 \mathrm{a}(15.0 \mathrm{mg}, 0.1 \mathrm{mmol}), 3 \mathrm{db}(29.3 \mathrm{mg}, 0.11 \mathrm{mmol})$ according to the general procedure for $40 \mathrm{~h}$ gave $4 \mathrm{adb}$ as a yellow solid $\left(37.1 \mathrm{mg}, 89 \%\right.$ yield, $99 \%$ ee). $\mathrm{mp}: 185-186{ }^{\circ} \mathrm{C}$, [a] ${ }_{D}^{20}=+132.4^{\circ}\left(\mathrm{C}=1.0, \mathrm{CH}_{2} \mathrm{Cl}_{2}\right)$; HPLC condition: chiralpak IA, $254 \mathrm{~nm}, 0.5 \mathrm{~mL} / \mathrm{min}$, hexane $/ \mathrm{i}-\mathrm{PrOH}=$ 94/6, $t_{\text {major }}=60.102 \mathrm{~min}, t_{\text {minor }}=69.200 \mathrm{~min} ;{ }^{1} \mathrm{H}$ NMR $\left(600 \mathrm{MHz}, \mathrm{CD}_{3} \mathrm{OD}\right) \delta 7.50-7.30(\mathrm{~m}, 2 \mathrm{H})$, $7.03(\mathrm{~d}, J=8.1 \mathrm{~Hz}, 1 \mathrm{H}), 3.99(\mathrm{~s}, 2 \mathrm{H}), 3.70(\mathrm{~d}, J=11.9 \mathrm{~Hz}, 3 \mathrm{H}), 3.43(\mathrm{~d}, J=11.9 \mathrm{~Hz}, 3 \mathrm{H}), 3.27$ (s, 3H), 1.18 (s, 3H) ppm. ${ }^{13} \mathrm{C}$ NMR $\left(151 \mathrm{MHz}, \mathrm{CD}_{3} \mathrm{OD}\right) \delta 174.4\left({ }^{3} \mathrm{~J}\left({ }^{13} \mathrm{C},{ }^{31} \mathrm{P}\right)=3.4 \mathrm{~Hz}\right), 155.6,142.5,129.8,127.8,127.6,125.4,109.6$, $61.1,57.2\left({ }^{2} J\left({ }^{13} \mathrm{C},{ }^{31} \mathrm{P}\right)=11.4 \mathrm{~Hz}\right), 52.6\left({ }^{2} J\left({ }^{13} \mathrm{C},{ }^{31} \mathrm{P}\right)=5.9 \mathrm{~Hz}\right), 52.5\left({ }^{2} J\left({ }^{13} \mathrm{C},{ }^{31} \mathrm{P}\right)=5.8 \mathrm{~Hz}\right), 45.5,25.7,13.3 \mathrm{ppm}$. HRMS (ESI-TOF) calcd. for $\mathrm{C}_{15} \mathrm{H}_{18} \mathrm{CIN}_{4} \mathrm{O}_{6} \mathrm{P}[\mathrm{M}+\mathrm{Na}]^{+}:$439.0550, found: 439.0550 . 
<smiles>COP(=O)(O)C(N)(C(=O)NC(C)(C)C)C1(C(=O)NC(C)(C)C)C(=O)N(C)c2ccc(Cl)cc21</smiles>

4aea

Reaction of $2 \mathrm{a}(15.0 \mathrm{mg}, 0.1 \mathrm{mmol})$, 3ea $(32.4 \mathrm{mg}, 0.11 \mathrm{mmol})$ according to the general procedure for $144 \mathrm{~h}$ gave 4 aea as a yellow solid $\left(27.1 \mathrm{mg}, 61 \%\right.$ yield, $99 \%$ ee) $. \mathrm{mp}: 147-148{ }^{\circ} \mathrm{C}$, $[\alpha]_{0}^{20}=+105.3^{\circ}\left(\mathrm{c}=1.0, \mathrm{CH}_{2} \mathrm{Cl}_{2}\right)$; HPLC condition: chiralpak IA, $254 \mathrm{~nm}, 0.5 \mathrm{~mL} / \mathrm{min}$, hexane/i$\mathrm{PrOH}=80 / 20, t_{\text {major }}=13.672 \mathrm{~min}, t_{\text {minor }}=20.442 \mathrm{~min} ;{ }^{1} \mathrm{H}$ NMR $\left(600 \mathrm{MHz}, \mathrm{CD}_{3} \mathrm{OD}\right) \delta 7.35(\mathrm{~d}, \mathrm{~J}=$ $7.9 \mathrm{~Hz}, 1 \mathrm{H}), 7.11(\mathrm{~d}, J=12.6 \mathrm{~Hz}, 2 \mathrm{H}), 3.68(\mathrm{~d}, J=11.9 \mathrm{~Hz}, 3 \mathrm{H}), 3.43(\mathrm{~d}, J=11.9 \mathrm{~Hz}, 3 \mathrm{H}), 3.26$ (s 3H), $1.46-1.12(\mathrm{~m}, 9 \mathrm{H}) \mathrm{ppm} .{ }^{13} \mathrm{C}$ NMR $\left(151 \mathrm{MHz}, \mathrm{CD}_{3} \mathrm{OD}\right) \delta 175.0\left({ }^{3} \mathrm{~J}\left({ }^{13} \mathrm{C},{ }^{31} \mathrm{P}\right)=3.4 \mathrm{~Hz}\right), 154.5,145.1,135.6,126.0,125.0,122.0$, 109.0, 80.4, $57.0\left({ }^{2} \mathrm{~J}\left({ }^{13} \mathrm{C},{ }^{31} \mathrm{P}\right)=10.7 \mathrm{~Hz}\right), 52.5\left({ }^{2} \mathrm{~J}\left({ }^{13} \mathrm{C},{ }^{31} \mathrm{P}\right)=5.8 \mathrm{~Hz}\right), 52.4\left({ }^{2} \mathrm{~J}\left({ }^{13} \mathrm{C},{ }^{31} \mathrm{P}\right)=5.7 \mathrm{~Hz}\right), 45.5,27.0,25.7 \mathrm{ppm} . \mathrm{HRMS}$ (ESI-TOF) calcd. for $\mathrm{C}_{17} \mathrm{H}_{22} \mathrm{CIN}_{4} \mathrm{O}_{6} \mathrm{P}[\mathrm{M}+\mathrm{Na}]^{+}: 467.0863$, found: 467.0867 .<smiles>CCOC(=O)NC1(C(N)=O)C(=O)N(C)c2cc(Cl)ccc21</smiles>

4aeb

(S)-ethyl (6-chloro-3-(diazo(dimethoxyphosphoryl)methyl)-1-methyl-2-oxoindolin-3-yl)carbamate (4aeb):

Reaction of $2 \mathrm{a}(15.0 \mathrm{mg}, 0.1 \mathrm{mmol}), 3 \mathrm{eb}(29.3 \mathrm{mg}, 0.11 \mathrm{mmol})$ according to the general procedure for $40 \mathrm{~h}$ gave 4 aeb as a yellow solid (35.8 $\mathrm{mg}, 86 \%$ yield, $99 \%$ ee). $\mathrm{mp}: 142-143{ }^{\circ} \mathrm{C}$, [a] ${ }_{\mathrm{D}}^{20}=+122.0^{\circ}\left(\mathrm{C}=1.0, \mathrm{CH}_{2} \mathrm{Cl}_{2}\right.$ ); HPLC condition: chiralpak IA, $254 \mathrm{~nm}, 0.5 \mathrm{~mL} / \mathrm{min}$, hexane/i-PrOH $=80 / 20, t_{\text {major }}=12.625 \mathrm{~min}, t_{\text {minor }}=15.059 \mathrm{~min} ;{ }^{1} \mathrm{H} \mathrm{NMR}\left(600 \mathrm{MHz}, \mathrm{CD}_{3} \mathrm{OD}\right) \delta 7.35(\mathrm{~d}, J=8.6 \mathrm{~Hz}$, 1H), $7.11(\mathrm{~d}, J=7.0 \mathrm{~Hz}, 2 \mathrm{H}), 4.06-3.87(\mathrm{~m}, 2 \mathrm{H}), 3.69(\mathrm{~d}, J=11.9 \mathrm{~Hz}, 3 \mathrm{H}), 3.44(\mathrm{~d}, J=11.9 \mathrm{~Hz}$ 3H), $3.26(\mathrm{~s}, 3 \mathrm{H}), 1.17(\mathrm{~s}, 3 \mathrm{H}) \mathrm{ppm} .{ }^{13} \mathrm{C}$ NMR $\left(151 \mathrm{MHz}, \mathrm{CD}_{3} \mathrm{OD}\right) \delta 174.8\left({ }^{3} \mathrm{~J}\left({ }^{13} \mathrm{C},{ }^{31} \mathrm{P}\right)=3.4 \mathrm{~Hz}\right), 155.5,145.2,135.7,126.1,124.6$, 122.0, 109.0, 61.0, $57.0\left({ }^{2} \mathrm{~J}\left({ }^{13} \mathrm{C},{ }^{31} \mathrm{P}\right)=11.4 \mathrm{~Hz}\right), 52.5\left({ }^{2} \mathrm{~J}\left({ }^{13} \mathrm{C},{ }^{31} \mathrm{P}\right)=5.8 \mathrm{~Hz}\right), 52.4\left({ }^{2} \mathrm{~J}\left({ }^{13} \mathrm{C},{ }^{31} \mathrm{P}\right)=5.8 \mathrm{~Hz}\right), 45.4,25.7,13.2 \mathrm{ppm}$ HRMS (ESI-TOF) calcd. for $\mathrm{C}_{15} \mathrm{H}_{18} \mathrm{CIN}_{4} \mathrm{O}_{6} \mathrm{P}[\mathrm{M}+\mathrm{Na}]^{+}:$439.0550, found: 3439.0553 .<smiles>COC(=O)C(N)(C(=O)NC(C)(C)C)[C@]1(NC(=O)OCc2ccccc2)C(=O)N(C)c2c(Cl)cccc21</smiles>

4afa

(S)-tert-butyl (7-chloro-3-(diazo(dimethoxyphosphoryl)methyl)-1-methyl-2-oxoindolin-3-yl)carbamate (4afa) :

Reaction of $2 \mathrm{a}(15.0 \mathrm{mg}, 0.1 \mathrm{mmol}), 3 \mathrm{fa}(32.4 \mathrm{mg}, 0.11 \mathrm{mmol})$ according to the general procedure for $69 \mathrm{~h}$ gave 4afa as a yellow solid $(25.8 \mathrm{mg}, 58 \%$ yield, $75 \%$ ee $) . \mathrm{mp}: 144-145^{\circ} \mathrm{C},[\mathrm{\alpha}]_{0}^{20}=+86.2^{\circ}(\mathrm{c}=1.0$, $\mathrm{CH}_{2} \mathrm{Cl}_{2}$ ); HPLC condition: chiralpak IA, $254 \mathrm{~nm}, 0.5 \mathrm{~mL} / \mathrm{min}$, hexane $/ i-\mathrm{PrOH}=80 / 20, t_{\text {major }}=18.971 \mathrm{~min}$, $t_{\text {minor }}=22.431 \mathrm{~min} ;{ }^{1} \mathrm{H}$ NMR $\left(600 \mathrm{MHz}, \mathrm{CD}_{3} \mathrm{OD}\right) \delta 7.36(\mathrm{~d}, J=8.1 \mathrm{~Hz}, 1 \mathrm{H}), 7.33-7.22(\mathrm{~m}, 1 \mathrm{H}), 7.08(\mathrm{t}$ $J=7.7 \mathrm{~Hz}, 1 \mathrm{H}), 3.67(\mathrm{~d}, J=11.9 \mathrm{~Hz}, 3 \mathrm{H}), 3.63(\mathrm{~s}, 3 \mathrm{H}), 3.45(\mathrm{~d}, J=11.9 \mathrm{~Hz}, 3 \mathrm{H}), 1.31(\mathrm{~s}, 9 \mathrm{H}) \mathrm{ppm} .{ }^{13} \mathrm{C}$ $\operatorname{NMR}\left(151 \mathrm{MHz}, \mathrm{CD}_{3} \mathrm{OD}\right) \delta 175.3\left({ }^{3} \mathrm{~J}\left({ }^{13} \mathrm{C},{ }^{31} \mathrm{P}\right)=3.3 \mathrm{~Hz}\right), 154.5,139.4,131.9,129.5,123.6,123.2,115.3,80.4,57.0\left({ }^{2} \mathrm{~J}\left({ }^{13} \mathrm{C},{ }^{31} \mathrm{P}\right)=\right.$ $8.4 \mathrm{~Hz}), 52.5\left({ }^{2} \mathrm{~J}\left({ }^{13} \mathrm{C},{ }^{31} \mathrm{P}\right)=1.4 \mathrm{~Hz}\right), 52.5\left({ }^{2} \mathrm{~J}\left({ }^{13} \mathrm{C},{ }^{31} \mathrm{P}\right)=1.5 \mathrm{~Hz}\right), 45.7,29.1,27.0$ ppm. HRMS (ESI-TOF) calcd. for $\mathrm{C}_{17} \mathrm{H}_{22} \mathrm{CIN}_{4} \mathrm{O}_{6} \mathrm{P}$ $[\mathrm{M}+\mathrm{Na}]^{+}:$467.0863, found: 467.0885 .<smiles>CCOC(=O)N[C@]1(C(N)=O)C(=O)N(C)c2c(Cl)cccc21</smiles>

$1.17(\mathrm{~s}, 3 \mathrm{H}) \mathrm{ppm} .{ }^{13} \mathrm{C}$ NMR $\left(151 \mathrm{MHz}, \mathrm{CD}_{3} \mathrm{OD}\right) \delta 175.2\left({ }^{3} \mathrm{~J}\left({ }^{13} \mathrm{C},{ }^{31} \mathrm{P}\right)=3.1 \mathrm{~Hz}\right), 155.5,139.5,132.0,129.1,123.7,123.2,115.4,61.1$ $57.0\left({ }^{2} \mathrm{~J}\left({ }^{13} \mathrm{C},{ }^{31} \mathrm{P}\right)=12.0 \mathrm{~Hz}\right), 52.5\left({ }^{2} \mathrm{~J}\left({ }^{13} \mathrm{C},{ }^{31} \mathrm{P}\right)=1.8 \mathrm{~Hz}\right), 52.5\left({ }^{2} \mathrm{~J}\left({ }^{13} \mathrm{C},{ }^{31} \mathrm{P}\right)=1.7 \mathrm{~Hz}\right), 45.7,29.1,13.2 \mathrm{ppm}$. HRMS (ESI-TOF) calcd. for $\mathrm{C}_{15} \mathrm{H}_{18} \mathrm{CIN}_{4} \mathrm{O}_{6} \mathrm{P}[\mathrm{M}+\mathrm{Na}]^{+}:$439.0550, found: 439.0554 
<smiles>COP(=O)(OC)C1(C(=N)O)C(=O)N(C)c2ccc(Br)cc21</smiles>

(S)-tert-butyl (5-bromo-3-(diazo(dimethoxyphosphoryl)methyl)-1-methyl-2-oxoindolin-3-yl)carbamate (4afa):

Reaction of $2 \mathrm{a}(15.0 \mathrm{mg}, 0.1 \mathrm{mmol}), 3 \mathrm{ga}(37.3 \mathrm{mg}, 0.11 \mathrm{mmol})$ according to the general procedure for $144 \mathrm{~h}$ gave 4 aga as a yellow solid $(40.1 \mathrm{mg}, 82 \%$ yield, $96 \% \mathrm{ee}) . \mathrm{mp}: 136-137{ }^{\circ} \mathrm{C}$, $[\alpha]_{0}^{20}=+115.3^{\circ}\left(\mathrm{c}=1.0, \mathrm{CH}_{2} \mathrm{Cl}_{2}\right)$; HPLC condition: chiralpak IA, $254 \mathrm{~nm}, 0.5 \mathrm{~mL} / \mathrm{min}$, hexane $/ \mathrm{i}$ $\mathrm{PrOH}=90 / 10, t_{\text {major }}=35.760 \mathrm{~min}, t_{\text {minor }}=41.100 \mathrm{~min} ;{ }^{1} \mathrm{H}$ NMR $\left(600 \mathrm{MHz}, \mathrm{CD}_{3} \mathrm{OD}\right) \delta 7.56(\mathrm{~d}, J=$

$8.1 \mathrm{~Hz}, 1 \mathrm{H}), 7.52(\mathrm{~d}, J=1.7 \mathrm{~Hz}, 1 \mathrm{H}), 6.97(\mathrm{~d}, J=8.3 \mathrm{~Hz}, 1 \mathrm{H}), 3.70(\mathrm{~d}, J=11.9 \mathrm{~Hz}, 3 \mathrm{H}), 3.42(\mathrm{~d}, J=11.9 \mathrm{~Hz}, 3 \mathrm{H}), 3.26(\mathrm{~s}, 3 \mathrm{H}), 1.31$ (s, 9H) ppm. ${ }^{13} \mathrm{C}$ NMR $\left(151 \mathrm{MHz}, \mathrm{CD}_{3} \mathrm{OD}\right) \delta 174.5\left({ }^{3} \mathrm{~J}\left({ }^{13} \mathrm{C},{ }^{31} \mathrm{P}\right)=3.6 \mathrm{~Hz}\right), 154.5,142.8,132.6,128.5,128.0,114.5,110.0,80.5,57.2$ $\left({ }^{2} J\left({ }^{13} \mathrm{C},{ }^{31} \mathrm{P}\right)=10.9 \mathrm{~Hz}\right), 52.6\left({ }^{2} \mathrm{~J}\left({ }^{13} \mathrm{C},{ }^{31} \mathrm{P}\right)=5.9 \mathrm{~Hz}\right), 52.4\left({ }^{2} \mathrm{~J}\left({ }^{13} \mathrm{C},{ }^{31} \mathrm{P}\right)=5.8 \mathrm{~Hz}\right), 45.6,27.0,25.6 \mathrm{ppm}$. HRMS (ESI-TOF) calcd. for $\mathrm{C}_{17} \mathrm{H}_{22} \mathrm{BrN}_{4} \mathrm{O}_{6} \mathrm{P}[\mathrm{M}+\mathrm{Na}]^{+}:$511.0358, found: 511.0362 .<smiles>CCOC(=O)NC1(C(=O)OC)C(=O)N(C)c2ccc(Br)cc21</smiles>

(S)-ethyl (5-bromo-3-(diazo(dimethoxyphosphoryl)methyl)-1-methyl-2-oxoindolin-3-yl)carbamate (4agb):

Reaction of $2 \mathrm{a}(15.0 \mathrm{mg}, 0.1 \mathrm{mmol}), 3 \mathrm{gb}(34.2 \mathrm{mg}, 0.11 \mathrm{mmol})$ according to the general procedure for $144 \mathrm{~h}$ gave 4 agb as a yellow solid (38.3 $\mathrm{mg}, 83 \%$ yield, $91 \%$ ee). $\mathrm{mp}: 196-198{ }^{\circ} \mathrm{C}$ $[\alpha]_{0}^{20}=+95.4^{\circ}\left(\mathrm{c}=1.0, \mathrm{CH}_{2} \mathrm{Cl}_{2}\right)$; HPLC condition: chiralpak IA, $254 \mathrm{~nm}, 0.5 \mathrm{~mL} / \mathrm{min}$, hexane $/ \mathrm{i}$-PrOH $=94 / 6, t_{\text {major }}=62.705 \mathrm{~min}, t_{\text {minor }}=71.082 \mathrm{~min} ;{ }^{1} \mathrm{H}$ NMR $\left(600 \mathrm{MHz}, \mathrm{CD}_{3} \mathrm{OD}\right) \delta 7.55(\mathrm{dd}, J=8.3,1.9$ $\mathrm{Hz}, 1 \mathrm{H}), 7.52(\mathrm{~d}, J=1.9 \mathrm{~Hz}, 1 \mathrm{H}), 6.98(\mathrm{~d}, J=8.3 \mathrm{~Hz}, 1 \mathrm{H}), 3.98(\mathrm{~d}, J=6.8 \mathrm{~Hz}, 2 \mathrm{H}), 3.70(\mathrm{~d}, J=11.9 \mathrm{~Hz}, 3 \mathrm{H}), 3.43(\mathrm{~d}, J=11.9 \mathrm{~Hz}$ 3H), $3.26(\mathrm{~s}, 3 \mathrm{H}), 1.18(\mathrm{~s}, 3 \mathrm{H}) \mathrm{ppm} .{ }^{13} \mathrm{C} \mathrm{NMR}\left(151 \mathrm{MHz}, \mathrm{CD}_{3} \mathrm{OD}\right) \delta 174.3\left({ }^{3} \mathrm{~J}\left({ }^{13} \mathrm{C},{ }^{31} \mathrm{P}\right)=3.5 \mathrm{~Hz}\right), .155 .6,142.9,132.7,132.3,132.3$, 131.7, 131.6, 128.6, 128.5, 128.2, 128.1, 114.6, 110.1, 61.1, $57.2\left({ }^{2} J\left({ }^{13} \mathrm{C},{ }^{31} \mathrm{P}\right)=11.4 \mathrm{~Hz}\right), 52.56\left({ }^{2} \mathrm{~J}\left({ }^{13} \mathrm{C},{ }^{31} \mathrm{P}\right)=5.9 \mathrm{~Hz}\right), 52.5\left({ }^{2} \mathrm{~J}\left({ }^{13} \mathrm{C}\right.\right.$, $\left.{ }^{31} \mathrm{P}\right)=5.9 \mathrm{~Hz}$ ), 45.5, 25.6, $13.2 \mathrm{ppm}$. HRMS (ESI-TOF) calcd. for $\mathrm{C}_{15} \mathrm{H}_{18} \mathrm{BrN}_{4} \mathrm{O}_{6} \mathrm{P}[\mathrm{M}+\mathrm{Na}]^{+}:$483.0045, found: 483.0047.

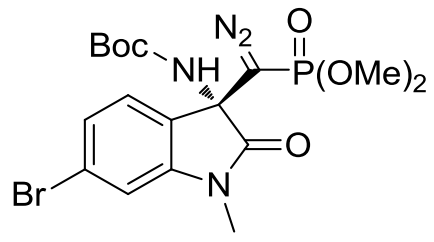

4aha

(S)-tert-butyl (6-bromo-3-(diazo(dimethoxyphosphoryl)methyl)-1-methyl-2-oxoindolin-3-yl)carbamate (4aha):

Reaction of $2 \mathrm{a}(15.0 \mathrm{mg}, 0.1 \mathrm{mmol})$, 3ha $(37.3 \mathrm{mg}, 0.11 \mathrm{mmol})$ according to the general procedure for $36 \mathrm{~h}$ gave 4 aha as a yellow solid (35.2 $\mathrm{mg}, 72 \%$ yield, $95 \%$ ee). $\mathrm{mp}: 141-142{ }^{\circ} \mathrm{C}$, [a] ${ }_{D}^{20}=+68.5^{\circ}\left(\mathrm{c}=1.0, \mathrm{CH}_{2} \mathrm{Cl}_{2}\right)$; HPLC condition: chiralpak IA, $254 \mathrm{~nm}, 0.5 \mathrm{~mL} / \mathrm{min}$, hexane $/ \mathrm{i}-\mathrm{PrOH}=$ 80/20, $t_{\text {major }}=13.259 \mathrm{~min}, t_{\text {minor }}=19.812 \mathrm{~min} ;{ }^{1} \mathrm{H}$ NMR $\left(600 \mathrm{MHz}, \mathrm{CD}_{3} \mathrm{OD}\right) \delta 7.27(\mathrm{dd}, J=21.7$, $10.3 \mathrm{~Hz}, 3 \mathrm{H}), 3.68$ (d, $J=11.9 \mathrm{~Hz}, 3 \mathrm{H}), 3.42(\mathrm{~d}, J=11.9 \mathrm{~Hz}, 3 \mathrm{H}), 3.26(\mathrm{~s}, 3 \mathrm{H}), 1.31(\mathrm{~s}, 9 \mathrm{H}) \mathrm{ppm} .{ }^{13} \mathrm{C} \mathrm{NMR}\left(151 \mathrm{MHz}, \mathrm{CD}_{3} \mathrm{OD}\right) \delta$ $174.85\left({ }^{3} \mathrm{~J}\left({ }^{13} \mathrm{C},{ }^{31} \mathrm{P}\right)=3.5 \mathrm{~Hz}\right), 154.5,145.1,126.3,125.5,125.0,123.3,111.8,57.0\left({ }^{2} \mathrm{~J}\left({ }^{13} \mathrm{C},{ }^{31} \mathrm{P}\right)=11.9 \mathrm{~Hz}\right), 52.5\left({ }^{2} J\left({ }^{13} \mathrm{C},{ }^{31} \mathrm{P}\right)=5.7\right.$ $\mathrm{Hz}), 52.4\left({ }^{2} \mathrm{~J}\left({ }^{13} \mathrm{C},{ }^{31} \mathrm{P}\right)=5.7 \mathrm{~Hz}\right), 45.3,27.0,25.7 \mathrm{ppm}$. HRMS (ESI-TOF) calcd. for $\mathrm{C}_{17} \mathrm{H}_{22} \mathrm{BrN}_{4} \mathrm{O}_{6} \mathrm{P}$ [M+Na] $]^{+}: 511.0358$, found: 511.0354 .<smiles>CCOC(=O)N[C@]1(C(=O)OC)C(=O)N(C)c2cc(Br)ccc21</smiles>

4ahb

(S)-ethyl (6-bromo-3-(diazo(dimethoxyphosphoryl)methyl)-1-methyl-2-oxoindolin-3-yl)carbamate (4ahb):

Reaction of $2 \mathrm{a}(15.0 \mathrm{mg}, 0.1 \mathrm{mmol}), 3 \mathrm{hb}(34.2 \mathrm{mg}, 0.11 \mathrm{mmol})$ according to the genera procedure for $24 \mathrm{~h}$ gave 4 ahb as a yellow solid (34.1 mg, 74\% yield, $98 \%$ ee). $\mathrm{mp}: 162-163{ }^{\circ} \mathrm{C}$ $[\alpha]_{0}^{20}=+128.1^{\circ}\left(\mathrm{c}=1.0, \mathrm{CH}_{2} \mathrm{Cl}_{2}\right)$; HPLC condition: chiralpak IA, $254 \mathrm{~nm}, 0.5 \mathrm{~mL} / \mathrm{min}$, hexane/i$\mathrm{PrOH}=94 / 6, t_{\text {major }}=50.988 \mathrm{~min}, t_{\text {minor }}=76.817 \mathrm{~min} ;{ }^{1} \mathrm{H}$ NMR $\left(600 \mathrm{MHz}, \mathrm{CD}_{3} \mathrm{OD}\right) \delta 7.31-7.17(\mathrm{~m}$, 3H), $3.94(\mathrm{~d}, J=6.5 \mathrm{~Hz}, 2 \mathrm{H}), 3.66(\mathrm{~d}, J=11.9 \mathrm{~Hz}, 3 \mathrm{H}), 3.41$ (d, $J=11.9 \mathrm{~Hz}, 3 \mathrm{H}), 3.23(\mathrm{~s}, 3 \mathrm{H}), 1.15(\mathrm{~s}, 3 \mathrm{H}) \mathrm{ppm} .{ }^{13} \mathrm{C} \mathrm{NMR}(151 \mathrm{MHz}$, $\left.\mathrm{CD}_{3} \mathrm{OD}\right) \delta 174.7\left({ }^{3} \mathrm{~J}\left({ }^{13} \mathrm{C},{ }^{31} \mathrm{P}\right)=3.3 \mathrm{~Hz}\right), 155.6,145.2,126.4,125.1,123.4,111.8,61.0,57.0\left({ }^{2} \mathrm{~J}\left({ }^{13} \mathrm{C},{ }^{31} \mathrm{P}\right)=11.4 \mathrm{~Hz}\right), 52.5\left({ }^{2} \mathrm{~J}\left({ }^{13} \mathrm{C}\right.\right.$ $\left.\left.{ }^{31} \mathrm{P}\right)=5.8 \mathrm{~Hz}\right), 52.4\left({ }^{2} \mathrm{~J}\left({ }^{13} \mathrm{C},{ }^{31} \mathrm{P}\right)=5.7 \mathrm{~Hz}\right), 45.3,25.7,13.2 \mathrm{ppm}$. HRMS (ESI-TOF) calcd. for $\mathrm{C}_{15} \mathrm{H}_{18} \mathrm{BrN}_{4} \mathrm{O}_{6} \mathrm{P}[\mathrm{M}+\mathrm{Na}]^{+}: 483.0045$, found: 483.0043 . 
<smiles>CO[P+](=O)[C@]1(NC(=O)OCc2ccccc2)C(=O)N(C)c2ccc(I)cc21</smiles>

4aia
(S)-tert-butyl (3-(diazo(dimethoxyphosphoryl)methyl)-5-iodo-1-methyl-2-oxoindolin-3-yl)carbamate (4aia):

Reaction of $2 \mathbf{a}(15.0 \mathrm{mg}, 0.1 \mathrm{mmol})$, 3ia $(42.5 \mathrm{mg}, 0.11 \mathrm{mmol})$ according to the general procedure for $36 \mathrm{~h}$ gave 4aia as a yellow solid $(34.8 \mathrm{mg}, 65 \%$ yield, $87 \%$ ee $) . \mathrm{mp}: 89-90^{\circ} \mathrm{C},[\mathrm{\alpha}]_{\mathrm{D}}^{20}=+82.9^{\circ}(\mathrm{c}=$ 1.0, $\mathrm{CH}_{2} \mathrm{Cl}_{2}$ ); HPLC condition: chiralpak IA, $254 \mathrm{~nm}, 0.5 \mathrm{~mL} / \mathrm{min}$, hexane $/ \mathrm{i}-\mathrm{PrOH}=94 / 6$, $t_{\text {major }}=$ $80.203 \mathrm{~min}, t_{\text {minor }}=93.969 \mathrm{~min} ;{ }^{1} \mathrm{H}$ NMR $\left(600 \mathrm{MHz}, \mathrm{CD}_{3} \mathrm{OD}\right) \delta 7.74(\mathrm{~d}, J=7.8 \mathrm{~Hz}, 1 \mathrm{H}), 7.68(\mathrm{~d}, J=$

$1.5 \mathrm{~Hz}, 1 \mathrm{H}), 6.86(\mathrm{~d}, J=8.2 \mathrm{~Hz}, 1 \mathrm{H}), 3.70(\mathrm{~d}, J=11.9 \mathrm{~Hz}, 3 \mathrm{H}), 3.41(\mathrm{~d}, J=11.9 \mathrm{~Hz}, 3 \mathrm{H}), 3.25(\mathrm{~s}, 3 \mathrm{H}), 1.47-1.15(\mathrm{~m}, 9 \mathrm{H}) \mathrm{ppm} .{ }^{13} \mathrm{C}$ $\operatorname{NMR}\left(151 \mathrm{MHz}, \mathrm{CD}_{3} \mathrm{OD}\right) \delta 174.3\left({ }^{3} \mathrm{~J}\left({ }^{13} \mathrm{C},{ }^{31} \mathrm{P}\right)=3.5 \mathrm{~Hz}\right), 154.5,143.4,138.6,133.8,128.7,110.5,83.8,80.5,57.0\left({ }^{2} \mathrm{~J}\left({ }^{13} \mathrm{C},{ }^{31} \mathrm{P}\right)=\right.$ $11.1 \mathrm{~Hz}), 52.6\left({ }^{2} J\left({ }^{13} \mathrm{C},{ }^{31} \mathrm{P}\right)=5.9 \mathrm{~Hz}\right), 52.5\left({ }^{2} \mathrm{~J}\left({ }^{13} \mathrm{C},{ }^{31} \mathrm{P}\right)=5.9 \mathrm{~Hz}\right), 45.6,27.0,25.6 \mathrm{ppm}$. HRMS (ESI-TOF) calcd. for $\mathrm{C}_{17} \mathrm{H}_{22} \mathrm{IN}_{4} \mathrm{O}_{6} \mathrm{P}$ $[\mathrm{M}+\mathrm{Na}]^{+}: 559.0219$, found: 559.0226 .<smiles>CCOC(=O)NC1(C(N)=O)C(=O)N(C)c2ccc(I)cc21</smiles>

4aib
(S)-ethyl (3-(diazo(dimethoxyphosphoryl)methyl)-5-iodo-1-methyl-2-oxoindolin-3-yl)carbamate (4aib):

Reaction of $2 \mathbf{a}(15.0 \mathrm{mg}, 0.1 \mathrm{mmol})$, $3 \mathbf{i b}(39.4 \mathrm{mg}, 0.11 \mathrm{mmol})$ according to the general procedure for $84 \mathrm{~h}$ gave 4aia as a yellow solid $\left(40.1 \mathrm{mg}, 79 \%\right.$ yield, $99 \%$ ee). $\mathrm{mp}: 209-210^{\circ} \mathrm{C},[\alpha]_{0}^{20}=+112.3^{\circ}$ ( $\mathrm{C}=1.0, \mathrm{CH}_{2} \mathrm{Cl}_{2}$ ); HPLC condition: chiralpak IA, $254 \mathrm{~nm}, 0.5 \mathrm{~mL} / \mathrm{min}$, hexane $/ i-\mathrm{PrOH}=94 / 6$, $t_{\text {major }}=$ $65.552 \mathrm{~min}, t_{\text {minor }}=75.733 \mathrm{~min} ;{ }^{1} \mathrm{H}$ NMR $\left(600 \mathrm{MHz}, \mathrm{CD}_{3} \mathrm{OD}\right) \delta 7.74(\mathrm{dd}, J=8.2,1.6 \mathrm{~Hz}, 1 \mathrm{H}), 7.68(\mathrm{~d}$,

$J=1.6 \mathrm{~Hz}, 1 \mathrm{H}), 6.87(\mathrm{~d}, J=8.2 \mathrm{~Hz}, 1 \mathrm{H}), 4.05-3.92(\mathrm{~m}, 2 \mathrm{H}), 3.70(\mathrm{~d}, J=11.8 \mathrm{~Hz}, 3 \mathrm{H}), 3.42(\mathrm{~d}, J=11.9 \mathrm{~Hz}, 3 \mathrm{H}), 3.25(\mathrm{~s}, 3 \mathrm{H}), 1.18$ (s, 3H) ppm. ${ }^{13} \mathrm{C}$ NMR $\left(151 \mathrm{MHz}, \mathrm{CD}_{3} \mathrm{OD}\right) \delta 174.2\left({ }^{3} \mathrm{~J}\left({ }^{13} \mathrm{C},{ }^{31} \mathrm{P}\right)=3.5 \mathrm{~Hz}\right), .155 .5,143.5,138.8,138.1,133.9,128.3,110.5,83.9,61.1$, $57.0\left({ }^{2} J\left({ }^{13} \mathrm{C},{ }^{31} \mathrm{P}\right)=11.4 \mathrm{~Hz}\right), 52.6\left({ }^{2} J\left({ }^{13} \mathrm{C},{ }^{31} \mathrm{P}\right)=6.0 \mathrm{~Hz}\right), 52.5\left({ }^{2} J\left({ }^{13} \mathrm{C},{ }^{31} \mathrm{P}\right)=5.9 \mathrm{~Hz}\right), 45.6,25.6,13.2 \mathrm{ppm}$. HRMS (ESI-TOF) calcd. for $\mathrm{C}_{15} \mathrm{H}_{18} \mathrm{IN}_{4} \mathrm{O}_{6} \mathrm{P}[\mathrm{M}+\mathrm{Na}]^{+}: 530.9906$, found: 530.9908 .<smiles>Cc1ccc2c(c1)[C@](NC(=O)OCc1ccccc1)(C(N)=O)C(=O)N2C</smiles>

4aja
(S)-tert-butyl (3-(diazo(dimethoxyphosphoryl)methyl)-5-iodo-1-methyl-2-oxoindolin-3-yl)carbamate (4aja):

Reaction of $2 \mathrm{a}(15.0 \mathrm{mg}, 0.1 \mathrm{mmol}), 3 \mathbf{j a}(30.1 \mathrm{mg}, 0.11 \mathrm{mmol})$ according to the general procedure for $12 \mathrm{~h}$ gave 4aia as a yellow solid $\left(28.0 \mathrm{mg}, 66 \% \text { yield, } 91 \% \text { ee). } \mathrm{mp}: 146-147^{\circ} \mathrm{C} \text {, [a }\right]_{\mathrm{D}}^{20}=+107.3^{\circ}$ ( $\mathrm{C}=1.0, \mathrm{CH}_{2} \mathrm{Cl}_{2}$ ); $\mathrm{HPLC}$ condition: chiralpak IA, $254 \mathrm{~nm}, 0.5 \mathrm{~mL} / \mathrm{min}$, hexane $/ \mathrm{i}-\mathrm{PrOH}=90 / 10$, $t_{\text {major }}=$ $36.088 \mathrm{~min}, t_{\text {minor }}=45.630 \mathrm{~min} ;{ }^{1} \mathrm{H}$ NMR $\left(600 \mathrm{MHz}, \mathrm{CD}_{3} \mathrm{OD}\right) \delta 7.22(\mathrm{~d}, J=6.1 \mathrm{~Hz}, 2 \mathrm{H}), 6.91(\mathrm{~d}, J=$

$8.3 \mathrm{~Hz}, 1 \mathrm{H}), 3.65$ (d, $J=11.9 \mathrm{~Hz}, 3 \mathrm{H}), 3.39$ (d, $J=11.9 \mathrm{~Hz}, 3 \mathrm{H}), 3.25(\mathrm{~s}, 3 \mathrm{H}), 2.36(\mathrm{~s}, 3 \mathrm{H}), 1.28(\mathrm{~s}, 9 \mathrm{H}) \mathrm{ppm} .{ }^{13} \mathrm{C} \mathrm{NMR}(151 \mathrm{MHz}$, $\left.\mathrm{CD}_{3} \mathrm{OD}\right) \delta 175.0\left({ }^{3} \mathrm{~J}\left({ }^{13} \mathrm{C},{ }^{31} \mathrm{P}\right)=3.4 \mathrm{~Hz}\right), 154.5,141.2,132.1,130.0,126.4,125.5,108.1,80.2,57.5\left({ }^{2} \mathrm{~J}\left({ }^{13} \mathrm{C},{ }^{31} \mathrm{P}\right)=10.9 \mathrm{~Hz}\right), 52.4\left({ }^{2} \mathrm{~J}\right.$ $\left.\left({ }^{13} \mathrm{C},{ }^{31} \mathrm{P}\right)=5.7 \mathrm{~Hz}\right), 52.4\left({ }^{2} \mathrm{~J}\left({ }^{13} \mathrm{C},{ }^{31} \mathrm{P}\right)=5.7 \mathrm{~Hz}\right), 45.7,27.0,25.6,19.7$ ppm. HRMS (ESI-TOF) calcd. for $\left.\mathrm{C}_{18} \mathrm{H}_{25} \mathrm{~N}_{4} \mathrm{O}_{6} \mathrm{P}[\mathrm{M}+\mathrm{Na}]\right]^{+}$: 447.1409, found: 447.1404 .<smiles>CCOC(=O)NC1(C(N)=O)C(=O)N(C)c2ccc(C)cc21</smiles>

(S)-ethyl (3-(diazo(dimethoxyphosphoryl)methyl)-1,5-dimethyl-2-oxoindolin-3-yl)carbamate (4ajb):

Reaction of $2 \mathrm{a}(15.0 \mathrm{mg}, 0.1 \mathrm{mmol}), 3 \mathrm{jb}(27.1 \mathrm{mg}, 0.11 \mathrm{mmol})$ according to the general procedure for $84 \mathrm{~h}$ gave 4 ajb as a yellow solid $\left(30.9 \mathrm{mg}, 78 \%\right.$ yield, $99 \%$ ee). mp: $170-171{ }^{\circ} \mathrm{C}$, $[\alpha]_{0}^{20}=+128.6^{\circ}$ ( $\mathrm{c}=1.0, \mathrm{CH}_{2} \mathrm{Cl}_{2}$ ); HPLC condition: chiralpak IA, $254 \mathrm{~nm}, 0.5 \mathrm{~mL} / \mathrm{min}$, hexane $/ i-\mathrm{PrOH}=90 / 10, t_{\text {major }}=$ $26.458 \mathrm{~min}, t_{\text {minor }}=33.706 \mathrm{~min} ;{ }^{1} \mathrm{H}$ NMR $\left(600 \mathrm{MHz}, \mathrm{CD}_{3} \mathrm{OD}\right) \delta 7.22(\mathrm{~d}, J=9.2 \mathrm{~Hz}, 2 \mathrm{H}), 6.91(\mathrm{~d}, J=$

$7.8 \mathrm{~Hz}, 1 \mathrm{H}), 4.03-3.89(\mathrm{~m}, 2 \mathrm{H}), 3.65(\mathrm{~d}, J=11.9 \mathrm{~Hz}, 3 \mathrm{H}), 3.41(\mathrm{~d}, J=11.9 \mathrm{~Hz}, 3 \mathrm{H}), 3.25(\mathrm{~s}, 3 \mathrm{H}), 2.35(\mathrm{~s}, 3 \mathrm{H}), 1.16(\mathrm{~s}, 3 \mathrm{H}) \mathrm{ppm} .{ }^{13} \mathrm{C}$ $\operatorname{NMR}\left(151 \mathrm{MHz}, \mathrm{CD}_{3} \mathrm{OD}\right) \delta 174.8\left({ }^{3} \mathrm{~J}\left({ }^{13} \mathrm{C},{ }^{31} \mathrm{P}\right)=3.3 \mathrm{~Hz}\right), 155.5,141.3,132.2,130.1,126.1,125.6,108.1,60.9,57.5\left({ }^{2} \mathrm{~J}\left({ }^{13} \mathrm{C},{ }^{31} \mathrm{P}\right)=\right.$ $11.5 \mathrm{~Hz}), 52.4\left({ }^{2} \mathrm{~J}\left({ }^{13} \mathrm{C},{ }^{31} \mathrm{P}\right)=5.9 \mathrm{~Hz}\right), 52.4\left({ }^{2} \mathrm{~J}\left({ }^{13} \mathrm{C},{ }^{31} \mathrm{P}\right)=5.8 \mathrm{~Hz}\right), 45.7,25.5,19.7,13.2 \mathrm{ppm}$. HRMS (ESI-TOF) calcd. for $\mathrm{C}_{16} \mathrm{H}_{21} \mathrm{~N}_{4} \mathrm{O}_{6} \mathrm{P}[\mathrm{M}+\mathrm{Na}]^{+}:$419.1096, found: 419.1093 . 
<smiles>COc1ccc2c(c1)C(C(=O)NC(C)(C)C)(C(=O)OCc1ccccc1)C(=O)N2C</smiles>

(S)-tert-butyl (3-(diazo(dimethoxyphosphoryl)methyl)-5-methoxy-1-methyl-2-oxoindolin-3-yl)carbamate (4aka): Reaction of 2a $(15.0 \mathrm{mg}, 0.1 \mathrm{mmol})$, 3ka $(31.9 \mathrm{mg}, 0.11 \mathrm{mmol})$ according to the general procedure for $12 \mathrm{~h}$ gave 4 ajb as a yellow solid (24.2 mg, 55\% yield, $43 \%$ ee). mp: $182-183{ }^{\circ} \mathrm{C}$, $[\alpha]_{0}^{20}=+16.5^{\circ}\left(\mathrm{c}=1.0, \mathrm{CH}_{2} \mathrm{Cl}_{2}\right)$; HPLC condition: chiralpak IA, $254 \mathrm{~nm}, 0.5 \mathrm{~mL} / \mathrm{min}$, hexane $/ \mathrm{i}-$ $\mathrm{PrOH}=80 / 20, t_{\text {major }}=18.817 \mathrm{~min}, t_{\text {minor }}=22.459 \mathrm{~min} ;{ }^{1} \mathrm{H}$ NMR $\left(600 \mathrm{MHz}, \mathrm{CD}_{3} \mathrm{OD}\right) \delta 7.01(\mathrm{~d}, J=$ $2.3 \mathrm{~Hz}, 1 \mathrm{H}$ ), 6.94 (dt, $J=15.9,5.2 \mathrm{~Hz}, 2 \mathrm{H}), 3.78$ (s, 3H), 3.66 (d, $J=12.0 \mathrm{~Hz}, 3 \mathrm{H}), 3.38$ (d, J = 12.0 Hz, 3H), $3.22(\mathrm{~s}, 3 \mathrm{H}), 1.25$ (dd, $J$ = 22.4, $15.2 \mathrm{~Hz}, 9 \mathrm{H}) \mathrm{ppm} .{ }^{13} \mathrm{C}$ NMR $\left(151 \mathrm{MHz}, \mathrm{CD}_{3} \mathrm{OD}\right) \delta 174.7\left({ }^{3} \mathrm{~J}\left({ }^{13} \mathrm{C},{ }^{31} \mathrm{P}\right)=3.5 \mathrm{~Hz}\right), 156.2,154.6,136.9,127.6,114.3,112.2$, 108.8, 80.2, $57.7\left({ }^{2} J\left({ }^{13} \mathrm{C},{ }^{31} \mathrm{P}\right)=10.5 \mathrm{~Hz}\right), 52.5\left({ }^{2} \mathrm{~J}\left({ }^{13} \mathrm{C},{ }^{31} \mathrm{P}\right)=5.7 \mathrm{~Hz}\right), 52.4\left({ }^{2} \mathrm{~J}\left({ }^{13} \mathrm{C},{ }^{31} \mathrm{P}\right)=5.8 \mathrm{~Hz}\right), 45.7,27.0,25.6 \mathrm{ppm} . \mathrm{HRMS}$ (ESI-TOF) calcd. for $\mathrm{C}_{18} \mathrm{H}_{25} \mathrm{~N}_{4} \mathrm{O}_{7} \mathrm{P}[\mathrm{M}+\mathrm{Na}]^{+}:$463.1359, found: 463.1364 .

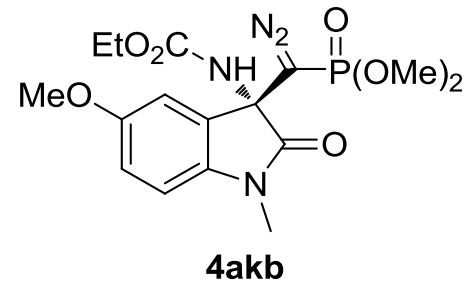

(S)-ethyl (3-(diazo(dimethoxyphosphoryl)methyl)-5-methoxy-1-methyl-2-oxoindolin-3-yl)carbamate (4akb): Reaction of $2 \mathrm{a}(15.0 \mathrm{mg}, 0.1 \mathrm{mmol}), 3 \mathbf{k b}(28.8 \mathrm{mg}, 0.11 \mathrm{mmol})$ according to the general procedure for $144 \mathrm{~h}$ gave 4 akb as a yellow solid (23.1 $\mathrm{mg}, 56 \%$ yield, $96 \%$ ee). $\mathrm{mp}: 138-139{ }^{\circ} \mathrm{C}$, $[\alpha]_{0}^{20}=+120.4^{\circ}\left(\mathrm{c}=1.0, \mathrm{CH}_{2} \mathrm{Cl}_{2}\right) ; \mathrm{HPLC}$ condition: chiralpak IA, $254 \mathrm{~nm}, 0.5 \mathrm{~mL} / \mathrm{min}$, hexane/i$\mathrm{PrOH}=80 / 20, t_{\text {major }}=15.087 \mathrm{~min}, t_{\text {minor }}=18.917 \mathrm{~min} ;{ }^{1} \mathrm{H} \mathrm{NMR}\left(600 \mathrm{MHz}, \mathrm{CD}_{3} \mathrm{OD}\right) \delta 7.01(\mathrm{~d}, J=$ $2.1 \mathrm{~Hz}, 1 \mathrm{H}), 6.97-6.88(\mathrm{~m}, 2 \mathrm{H}), 3.93(\mathrm{t}, J=16.0 \mathrm{~Hz}, 2 \mathrm{H}), 3.78(\mathrm{~s}, 3 \mathrm{H}), 3.66(\mathrm{~d}, J=12.0 \mathrm{~Hz}, 3 \mathrm{H}), 3.38(\mathrm{~d}, J=12.0 \mathrm{~Hz}, 3 \mathrm{H}), 3.22(\mathrm{~s}$, 3H), $1.16(\mathrm{~s}, 3 \mathrm{H}) \mathrm{ppm} .{ }^{13} \mathrm{C}$ NMR $\left(151 \mathrm{MHz}, \mathrm{CD}_{3} \mathrm{OD}\right) \delta 174.6\left({ }^{3} \mathrm{~J}\left({ }^{13} \mathrm{C},{ }^{31} \mathrm{P}\right)=3.4 \mathrm{~Hz}\right), 156.2,155.5,137.0,127.2,114.4,112.3,108.8$, 61.0, $57.7\left({ }^{2} \mathrm{~J}\left({ }^{13} \mathrm{C},{ }^{31} \mathrm{P}\right)=11.4 \mathrm{~Hz}\right), 52.5\left({ }^{2} \mathrm{~J}\left({ }^{13} \mathrm{C},{ }^{31} \mathrm{P}\right)=5.8 \mathrm{~Hz}\right), 52.4\left({ }^{2} \mathrm{~J}\left({ }^{13} \mathrm{C},{ }^{31} \mathrm{P}\right)=5.8 \mathrm{~Hz}\right)$, 52.4, 45.6, 25.6, 13.3 ppm. HRMS (ESITOF) calcd. for $\mathrm{C}_{16} \mathrm{H}_{21} \mathrm{~N}_{4} \mathrm{O}_{7} \mathrm{P}[\mathrm{M}+\mathrm{Na}]^{+}:$435.1046, found: 435.1046 .<smiles>COP(C)(=O)C(=O)[C@]1(NC(=O)OCc2ccccc2)C(=O)N(C)c2c(C)cc(Br)cc21</smiles>

4ala

(S)-tert-butyl (5-bromo-3-(diazo(dimethoxyphosphoryl)methyl)-1,7-dimethyl-2-oxoindolin-3-yl)carbamate (4ala):

Reaction of $2 \mathrm{a}(15.0 \mathrm{mg}, 0.1 \mathrm{mmol})$, 3la $(38.8 \mathrm{mg}, 0.11 \mathrm{mmol})$ according to the general procedure for $36 \mathrm{~h}$ gave 4 ala as a yellow solid $\left(38.2 \mathrm{mg}, 76 \% \text { yield, } 69 \% \text { ee). } \mathrm{mp}: 76-77^{\circ} \mathrm{C} \text {, [a }\right]_{\square}^{20}$ $=+18.6^{\circ}\left(\mathrm{c}=1.0, \mathrm{CH}_{2} \mathrm{Cl}_{2}\right)$; HPLC condition: chiralpak ODH, $254 \mathrm{~nm}, 0.5 \mathrm{~mL} / \mathrm{min}$, hexane $/ i-\mathrm{PrOH}$ $=70 / 30, t_{\text {major }}=9.696 \mathrm{~min}, t_{\text {minor }}=11.058 \mathrm{~min} ;{ }^{1} \mathrm{H} \mathrm{NMR}\left(600 \mathrm{MHz}, \mathrm{CD}_{3} \mathrm{OD}\right) \delta 7.33(\mathrm{~s}, 2 \mathrm{H}), 3.69(\mathrm{~d}$, $J=11.9 \mathrm{~Hz}, 3 \mathrm{H}), 3.54(\mathrm{~s}, 3 \mathrm{H}), 3.45(\mathrm{~d}, J=11.9 \mathrm{~Hz}, 3 \mathrm{H}), 2.60(\mathrm{~s}, 3 \mathrm{H}), 1.32(\mathrm{~s}, 9 \mathrm{H}) \mathrm{ppm} .{ }^{13} \mathrm{C} \mathrm{NMR}\left(151 \mathrm{MHz}, \mathrm{CD}_{3} \mathrm{OD}\right) \delta 175.2\left({ }^{3} J\left({ }^{13} \mathrm{C}\right.\right.$, $\left.\left.{ }^{31} \mathrm{P}\right)=3.4 \mathrm{~Hz}\right), 154.5,140.5,135.6,129.1,125.7,122.6,114.3,80.5,56.8\left({ }^{2} \mathrm{~J}\left({ }^{13} \mathrm{C},{ }^{31} \mathrm{P}\right)=11.5 \mathrm{~Hz}\right), 52.6\left({ }^{2} \mathrm{~J}\left({ }^{13} \mathrm{C},{ }^{31} \mathrm{P}\right)=5.9 \mathrm{~Hz}\right), 52.5$ $\left({ }^{2} J\left({ }^{13} \mathrm{C},{ }^{31} \mathrm{P}\right)=5.8 \mathrm{~Hz}\right), 45.8,29.0,27.1,17.3 \mathrm{ppm}$. HRMS (ESI-TOF) calcd. for $\mathrm{C}_{18} \mathrm{H}_{24} \mathrm{BrN}_{4} \mathrm{O}_{6} \mathrm{P}[\mathrm{M}+\mathrm{Na}]^{+}: 525.0515$, found: 525.0508 .<smiles>CCOC(=O)N[C@@]1(C(=N)OP(C)C)C(=O)N(C)c2c(C)cc(Br)cc21</smiles>

4alb

(S)-ethyl (5-bromo-3-(diazo(dimethoxyphosphoryl)methyl)-1,7-dimethyl-2-oxoindolin-3-yl)carbamate (4alb):

Reaction of $2 \mathrm{a}(15.0 \mathrm{mg}, 0.1 \mathrm{mmol})$, 3la $(35.8 \mathrm{mg}, 0.11 \mathrm{mmol})$ according to the general procedure for $67 \mathrm{~h}$ gave 4 alb as a yellow solid (38.0 mg, 80\% yield, $80 \%$ ee). mp: $120-121^{\circ} \mathrm{C}$, [ $\mathrm{\alpha}$ ] ${ }_{D}^{20}=+171.7^{\circ}\left(\mathrm{c}=1.0, \mathrm{CH}_{2} \mathrm{Cl}_{2}\right)$; HPLC condition: chiralpak IA, $254 \mathrm{~nm}, 0.5 \mathrm{~mL} / \mathrm{min}$, hexane/i-PrOH $=90 / 10, t_{\text {major }}=24.969 \mathrm{~min}, t_{\text {minor }}=29.790 \mathrm{~min} ;{ }^{1} \mathrm{H} \mathrm{NMR}\left(600 \mathrm{MHz}, \mathrm{CD}_{3} \mathrm{OD}\right) \delta 7.33(\mathrm{~s}, 2 \mathrm{H}), 4.04-$ $3.94(\mathrm{~m}, 2 \mathrm{H}), 3.69(\mathrm{~d}, J=11.9 \mathrm{~Hz}, 3 \mathrm{H}), 3.54(\mathrm{~s}, 3 \mathrm{H}), 3.46(\mathrm{~d}, J=11.9 \mathrm{~Hz}, 3 \mathrm{H}), 2.60$ (s, 3H), 1.19 (s, 3H) ppm. ${ }^{13} \mathrm{C}$ NMR $\left(151 \mathrm{MHz}, \mathrm{CD}_{3} \mathrm{OD}\right) \delta 175.1\left({ }^{3} \mathrm{~J}\left({ }^{13} \mathrm{C},{ }^{31} \mathrm{P}\right)=3.3 \mathrm{~Hz}\right), 155.5,140.6,135.7,128.7,125.9,122.6,114.3,61.1,56.8$ $\left({ }^{2} J\left({ }^{13} \mathrm{C},{ }^{31} \mathrm{P}\right)=11.4 \mathrm{~Hz}\right), 52.6\left({ }^{2} \mathrm{~J}\left({ }^{13} \mathrm{C},{ }^{31} \mathrm{P}\right)=6.0 \mathrm{~Hz}\right), 52.5\left({ }^{2} \mathrm{~J}\left({ }^{13} \mathrm{C},{ }^{31} \mathrm{P}\right)=5.9 \mathrm{~Hz}\right), 45.8,29.0,17.3,13.3 \mathrm{ppm}$. HRMS (ESI-TOF) calcd. for $\mathrm{C}_{16} \mathrm{H}_{20} \mathrm{BrN}_{4} \mathrm{O}_{6} \mathrm{P}[\mathrm{M}+\mathrm{Na}]^{+}:$497.0202, found: 497.0205 . 
<smiles>CN1C(=O)[C@](NC(=O)OCc2ccccc2)(C(N)=O)c2cc(OC(F)(F)F)ccc21</smiles>

4ama
(S)-tert-butyl (3-(diazo(dimethoxyphosphoryl)methyl)-1-methyl-2-oxo-5-(trifluoromethoxy)ind olin-3-yl)carbamate (4ama):

Reaction of $2 \mathrm{a}(15.0 \mathrm{mg}, 0.1 \mathrm{mmol}), 3 \mathrm{ma}(37.8 \mathrm{mg}, 0.11 \mathrm{mmol})$ according to the general procedure for $24 \mathrm{~h}$ gave 4 ama as a yellow solid $(44.0 \mathrm{mg}, 89 \%$ yield, $95 \%$ ee). $\mathrm{mp}: 128$ $129^{\circ} \mathrm{C},[\alpha]_{0}^{20}=+73.4^{\circ}\left(\mathrm{c}=1.0, \mathrm{CH}_{2} \mathrm{Cl}_{2}\right)$; HPLC condition: chiralpak IA, $254 \mathrm{~nm}, 0.5 \mathrm{~mL} / \mathrm{min}$

hexane $/ \mathrm{i}-\mathrm{PrOH}=94 / 6, t_{\text {major }}=46.107 \mathrm{~min}, t_{\text {minor }}=53.700 \mathrm{~min} ;{ }^{1} \mathrm{H} \mathrm{NMR}\left(600 \mathrm{MHz}, \mathrm{CD}_{3} \mathrm{OD}\right) \delta 7.34(\mathrm{~d}, J=8.2 \mathrm{~Hz}, 1 \mathrm{H}), 7.11(\mathrm{~d}, J=8.3$ $\mathrm{Hz}, 1 \mathrm{H}), 3.71(\mathrm{~d}, J=11.9 \mathrm{~Hz}, 1 \mathrm{H}), 3.38(\mathrm{~d}, J=11.9 \mathrm{~Hz}, 1 \mathrm{H}), 3.29(\mathrm{~s}, 1 \mathrm{H}), 1.32(\mathrm{~d}, J=10.2 \mathrm{~Hz}, 1 \mathrm{H}) \mathrm{ppm} .{ }^{13} \mathrm{C} \mathrm{NMR}\left(151 \mathrm{MHz}, \mathrm{CD}_{3} \mathrm{OD}\right)$ $\delta 174.9\left({ }^{3} \mathrm{~J}\left({ }^{13} \mathrm{C},{ }^{31} \mathrm{P}\right)=3.6 \mathrm{~Hz}\right), 154.5,144.4,142.6,128.0,123.2,123.0,121.5,119.8,118.7,118.1,109.1,80.4,57.3\left({ }^{2} \mathrm{~J}\left({ }^{13} \mathrm{C},{ }^{31} \mathrm{P}\right)=\right.$ $12.0 \mathrm{~Hz}), 52.4\left({ }^{2} \mathrm{~J}\left({ }^{13} \mathrm{C},{ }^{31} \mathrm{P}\right)=5.7 \mathrm{~Hz}\right), 52.3\left({ }^{2} \mathrm{~J}\left({ }^{13} \mathrm{C},{ }^{31} \mathrm{P}\right)=5.8 \mathrm{~Hz}\right) ., 45.4,27.0,25.7 \mathrm{ppm}$. HRMS (ESI-TOF) calcd. for $\mathrm{C}_{18} \mathrm{H}_{22} \mathrm{~F}_{3} \mathrm{~N}_{4} \mathrm{O}_{7} \mathrm{P}$ $[\mathrm{M}+\mathrm{Na}]^{+}: 517.1076$, found: 517.1075 .<smiles>CCOC(=O)NC1(C(N)=O)C(=O)N(C)c2ccc(OC(F)(F)F)cc21</smiles>

$4 \mathrm{amb}$

(S)-ethyl (3-(diazo(dimethoxyphosphoryl)methyl)-1-methyl-2-oxo-5- (trifluoromethoxy)ind olin-3-yl)carbamate (4amb):

Reaction of 2a $(15.0 \mathrm{mg}, 0.1 \mathrm{mmol}), 3 \mathrm{mb}(34.8 \mathrm{mg}, 0.11 \mathrm{mmol})$ according to the general procedure for $144 \mathrm{~h}$ gave $4 \mathrm{amb}$ as a yellow solid (33.1 mg, $71 \%$ yield, $95 \%$ ee). $\mathrm{mp}$ : 141 $142{ }^{\circ} \mathrm{C},[\alpha]_{0}^{20}=+91.5^{\circ}\left(\mathrm{c}=1.0, \mathrm{CH}_{2} \mathrm{Cl}_{2}\right)$; HPLC condition: chiralpak IA, $254 \mathrm{~nm}, 0.5 \mathrm{~mL} / \mathrm{min}$ hexane $/ \mathrm{i}-\mathrm{PrOH}=94 / 6, t_{\text {major }}=34.933 \mathrm{~min}, t_{\text {minor }}=42.950 \mathrm{~min} ;{ }^{1} \mathrm{H} \mathrm{NMR}\left(600 \mathrm{MHz}, \mathrm{CD}_{3} \mathrm{OD}\right) \delta$ $7.31(\mathrm{~s}, 2 \mathrm{H}), 7.11-7.05(\mathrm{~m}, 1 \mathrm{H}), 3.96(\mathrm{~s}, 2 \mathrm{H}), 3.69(\mathrm{~d}, J=11.9 \mathrm{~Hz}, 3 \mathrm{H}), 3.36(\mathrm{~d}, J=11.9 \mathrm{~Hz}, 3 \mathrm{H}), 3.27(\mathrm{~s}, 3 \mathrm{H}), 1.16(\mathrm{~s}, 3 \mathrm{H}) \mathrm{ppm} .{ }^{13} \mathrm{C}$ $\operatorname{NMR}\left(151 \mathrm{MHz}, \mathrm{CD}_{3} \mathrm{OD}\right) \delta 174.7\left({ }^{3} \mathrm{~J}\left({ }^{13} \mathrm{C},{ }^{31} \mathrm{P}\right)=3.5 \mathrm{~Hz}\right), 155.6,144.5,144.5,142.7,127.6,123.2,123.1,121.5,119.8,118.9,118.1$, 109.1, 61.1, $57.3\left({ }^{2} \mathrm{~J}\left({ }^{13} \mathrm{C},{ }^{31} \mathrm{P}\right)=11.3 \mathrm{~Hz}\right), 52.5\left({ }^{2} \mathrm{~J}\left({ }^{13} \mathrm{C},{ }^{31} \mathrm{P}\right)=5.7 \mathrm{~Hz}\right), 52.3\left({ }^{2} \mathrm{~J}\left({ }^{13} \mathrm{C},{ }^{31} \mathrm{P}\right)=5.8 \mathrm{~Hz}\right), 45.4,25.7,13.2 \mathrm{ppm} . \mathrm{HRMS}$ (ESI-TOF) calcd. for $\mathrm{C}_{16} \mathrm{H}_{18} \mathrm{~F}_{3} \mathrm{~N}_{4} \mathrm{O}_{7} \mathrm{P}[\mathrm{M}+\mathrm{Na}]^{+}:$489.0763, found: 489.0764<smiles>CO[P+](=O)C(=N)[C@]1(NC(=O)OCc2ccccc2)C(=O)N(C)c2ccc([N+](=O)[O-])cc21</smiles>

4ana

(S)-tert-butyl (3-(diazo(dimethoxyphosphoryl)methyl)-1-methyl-5-nitro-2-oxoindolin-3yl)carbamate (4ana):

Reaction of 2a $(15.0 \mathrm{mg}, 0.1 \mathrm{mmol})$, 3na $(33.6 \mathrm{mg}, 0.11 \mathrm{mmol})$ according to the general procedure for $108 \mathrm{~h}$ gave 4 ana as a yellow solid (38.2 mg, $84 \%$ yield, $85 \%$ ee). $\mathrm{mp}: 81-82{ }^{\circ} \mathrm{C}$ $[\alpha]_{D}^{20}=+68.1^{\circ}\left(\mathrm{c}=1.0, \mathrm{CH}_{2} \mathrm{Cl}_{2}\right)$; HPLC condition: chiralpak IA, $254 \mathrm{~nm}, 0.5 \mathrm{~mL} / \mathrm{min}$, hexane $/ \mathrm{i}$ $\mathrm{PrOH}=94 / 6, t_{\text {major }}=96.246 \mathrm{~min}, t_{\text {minor }}=107.860 \mathrm{~min} ;{ }^{1} \mathrm{H}$ NMR $\left(600 \mathrm{MHz}, \mathrm{CD}_{3} \mathrm{OD}\right) \delta 8.45-8.33(\mathrm{~m}, 1 \mathrm{H}), 8.26(\mathrm{~d}, J=2.3 \mathrm{~Hz}, 1 \mathrm{H})$, $7.23(\mathrm{~d}, J=8.7 \mathrm{~Hz}, 1 \mathrm{H}), 3.72(\mathrm{~d}, J=11.9 \mathrm{~Hz}, 3 \mathrm{H}), 3.40(\mathrm{~d}, J=11.9 \mathrm{~Hz}, 3 \mathrm{H}), 3.36(\mathrm{~s}, 3 \mathrm{H}), 1.34(\mathrm{~s}, 9 \mathrm{H}) \mathrm{ppm} .{ }^{13} \mathrm{C} \mathrm{NMR}(151 \mathrm{MHz}$, $\left.\mathrm{CD}_{3} \mathrm{OD}\right) \delta 175.1\left({ }^{3} \mathrm{~J}\left({ }^{13} \mathrm{C},{ }^{31} \mathrm{P}\right)=3.6 \mathrm{~Hz}\right), 154.7,149.3,143.2,131.7,131.6,128.61,128.5,127.2,126.6,120.5,108.4,80.6,56.8\left({ }^{2} \mathrm{~J}\right.$ $\left.\left({ }^{13} \mathrm{C},{ }^{31} \mathrm{P}\right)=12.5 \mathrm{~Hz}\right), 52.6\left({ }^{2} \mathrm{~J}\left({ }^{13} \mathrm{C},{ }^{31} \mathrm{P}\right)=5.9 \mathrm{~Hz}\right), 52.4\left({ }^{2} \mathrm{~J}\left({ }^{13} \mathrm{C},{ }^{31} \mathrm{P}\right)=5.8 \mathrm{~Hz}\right), 45.2,27.0,26.0 \mathrm{ppm}$. HRMS (ESI-TOF) calcd. for $\mathrm{C}_{17} \mathrm{H}_{22} \mathrm{~N}_{5} \mathrm{O}_{8} \mathrm{P}[\mathrm{M}+\mathrm{Na}]^{+}: 478.1104$, found: 478.1108 .<smiles>CCOC(=N)[C@@]1(NC(=O)OCC)C(=O)N(C)c2ccc([N+](=O)[O-])cc21</smiles>

4anb

(S)-ethyl (3-(diazo(dimethoxyphosphoryl)methyl)-1-methyl-5-nitro-2-oxoindolin-3-yl)carbamate (4anb): Reaction of 2a $(15.0 \mathrm{mg}, 0.1 \mathrm{mmol}), 3 \mathrm{nb}(30.5 \mathrm{mg}, 0.11 \mathrm{mmol})$ according to the general procedure for $144 \mathrm{~h}$ gave $4 \mathrm{anb}$ as a yellow solid (25.2 mg, $59 \%$ yield, $60 \%$ ee). mp: $180-181^{\circ} \mathrm{C}$ $[\alpha]_{0}^{20}=+51.0^{\circ}\left(\mathrm{c}=1.0, \mathrm{CH}_{2} \mathrm{Cl}_{2}\right) ; \mathrm{HPLC}$ condition: chiralpak IA, $254 \mathrm{~nm}, 0.5 \mathrm{~mL} / \mathrm{min}$, hexane $/ \mathrm{i}$ $\mathrm{PrOH}=90 / 10, t_{\text {minor }}=37.785 \mathrm{~min}, t_{\text {major }}=42.253 \mathrm{~min} ;{ }^{1} \mathrm{H}$ NMR $\left(600 \mathrm{MHz}, \mathrm{CD}_{3} \mathrm{OD}\right) \delta 8.38(\mathrm{dd}, J$ $=8.7,2.3 \mathrm{~Hz}, 1 \mathrm{H}), 8.26(\mathrm{~d}, J=2.3 \mathrm{~Hz}, 1 \mathrm{H}), 7.24(\mathrm{~d}, J=8.7 \mathrm{~Hz}, 1 \mathrm{H}), 4.10-3.93(\mathrm{~m}, 2 \mathrm{H}), 3.72(\mathrm{~d}$, 
$J=11.9 \mathrm{~Hz}, 3 \mathrm{H}), 3.41(\mathrm{~d}, J=11.9 \mathrm{~Hz}, 3 \mathrm{H}), 3.36(\mathrm{~s}, 3 \mathrm{H}), 1.19(\mathrm{~s}, 3 \mathrm{H}) \mathrm{ppm} .{ }^{13} \mathrm{C} \mathrm{NMR}\left(151 \mathrm{MHz}, \mathrm{CD}_{3} \mathrm{OD}\right) \delta 175.0\left({ }^{3} J\left({ }^{13} \mathrm{C},{ }^{31} \mathrm{P}\right)=3.6\right.$ $\mathrm{Hz})$, , 155.6, 149.4, 143.2, 126.8, 126.7, 120.7, 108.4, 61.3, $56.8\left({ }^{2} \mathrm{~J}\left({ }^{13} \mathrm{C},{ }^{31} \mathrm{P}\right)=11.3 \mathrm{~Hz}\right), 52.6\left({ }^{2} \mathrm{~J}\left({ }^{13} \mathrm{C},{ }^{31} \mathrm{P}\right)=5.8 \mathrm{~Hz}\right), 52.5\left({ }^{2} \mathrm{~J}\left({ }^{13} \mathrm{C}\right.\right.$, $\left.\left.{ }^{31} \mathrm{P}\right)=5.8 \mathrm{~Hz}\right) ., 45.2,26.0,13.2 \mathrm{ppm}$. HRMS (ESI-TOF) calcd. for $\mathrm{C}_{15} \mathrm{H}_{18} \mathrm{~N}_{5} \mathrm{O}_{8} \mathrm{P}[\mathrm{M}+\mathrm{Na}]^{+}: 450.0791$, found: 450.0781 .

\section{Hydrogenation of (S)-tert-butyl (3-(diazo(dimethoxyphosphoryl)methyl)-1-methyl-2-}

\section{oxoindolin-3-yl)carbamate (4aaa) into $1 a^{5}$}

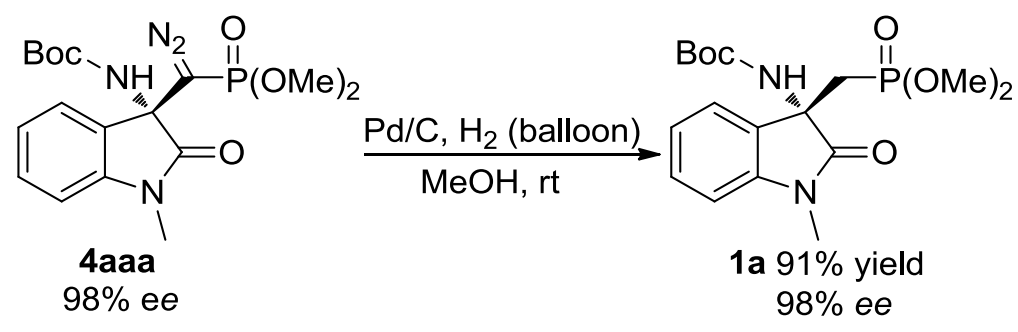

To a solution of (S)-tert-butyl (3-(diazo(dimethoxyphosphoryl)methyl)-1-methyl-2-oxoindolin-3-yl)carbamate (41.0 mg, $0.1 \mathrm{mmol})$ in $\mathrm{MeOH}(2.0 \mathrm{~mL})$ was added $10 \% \mathrm{Pd} / \mathrm{C}(1.06 \mathrm{mg}, 1 \mathrm{~mol} \%)$. The reaction vessel was purged with $\mathrm{H}_{2}(3$ times), then put under $1 \mathrm{~atm}$ of $\mathrm{H}_{2}$ (balloon). The reaction mixture was stirred for $48 \mathrm{~h}$ at $\mathrm{rt}$, filtrated through a plug of celite, then the filtrate was concentrated under vacuum, the resulting residue was purified by flash chromatography using (PE/EA = 1/1) to give the title compound in $91 \%$ yield $(98 \%$ ee $)$ as white solid. $\mathrm{mp}: 166-167{ }^{\circ} \mathrm{C},[\mathrm{\alpha}]_{0}^{20}=-24.1^{\circ}\left(\mathrm{c}=1.0, \mathrm{CH}_{2} \mathrm{Cl}_{2}\right)$; HPLC condition: chiralpak IA, $254 \mathrm{~nm}, 0.5 \mathrm{~mL} / \mathrm{min}$, hexane $/ \mathrm{i}-\mathrm{PrOH}=70 / 30, t_{\text {major }}=12.609 \mathrm{~min}, t_{\text {minor }}=19.630 \mathrm{~min} ;{ }^{1} \mathrm{H} \mathrm{NMR}\left(600 \mathrm{MHz}, \mathrm{CDCl}_{3}\right) \delta 7.48(\mathrm{~d}, J=7.4 \mathrm{~Hz}, 1 \mathrm{H}), 7.30(\mathrm{t}, J=6.2$ Hz, 1H), 7.05 (t, J=7.5 Hz, 1H), 6.83 (d, J = 7.8 Hz, 1H), 3.66 (d, J = 11.0 Hz, 3H), 3.60 (d, J = 11.2 Hz, 3H), 3.23 (s, 3H), 2.50 (t, J= $15.9 \mathrm{~Hz}, 1 \mathrm{H}), 2.00$ (dd, $J=18.9,15.4 \mathrm{~Hz}, 1 \mathrm{H}), 1.24(\mathrm{~s}, 9 \mathrm{H}) \mathrm{ppm} .{ }^{13} \mathrm{C} \mathrm{NMR}\left(151 \mathrm{MHz}, \mathrm{CDCl}_{3}\right) \delta 176.0\left({ }^{3} J\left({ }^{13} \mathrm{C},{ }^{31} \mathrm{P}\right)=14.5 \mathrm{~Hz}\right), 153.7$, 143.2, 129.5, 129.2, 124.2, 122.3, 108.2, 80.2, 58.3, $52.9\left({ }^{2} J\left({ }^{13} \mathrm{C},{ }^{31} \mathrm{P}\right)=6.2 \mathrm{~Hz}\right), 52.2\left({ }^{2} \mathrm{~J}\left({ }^{13} \mathrm{C},{ }^{31} \mathrm{P}\right)=6.9 \mathrm{~Hz}\right), 31.9\left({ }^{1} \mathrm{~J}\left({ }^{13} \mathrm{C},{ }^{31} \mathrm{P}\right)=\right.$ 141.4 Hz), 28.0, 26.6 ppm. HRMS (ESI-TOF) calcd. for $\mathrm{C}_{17} \mathrm{H}_{25} \mathrm{~N}_{2} \mathrm{O}_{6} \mathrm{P}[\mathrm{M}+\mathrm{Na}]^{+}: 407.1348$, found: 407.1340 .

\section{Synthesis of (S)-tert-butyl (3-(dimethoxyphosphoryl)-4-(2-(methylamino)phenyl)-5-oxo-4,5-}

\section{dihydro-1H-pyrazol-4-yl)carbamate $9^{6}$.}

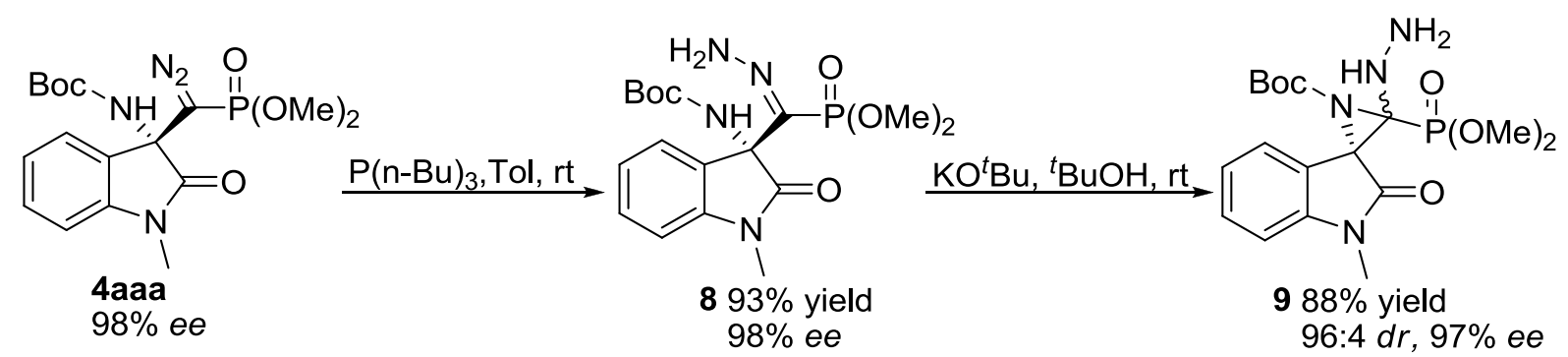

To a solution of (S)-tert-butyl (3-(diazo(dimethoxyphosphoryl)methyl)-1-methyl-2-oxoindolin-3-yl)carbamate 4aaa (41.0 mg, 0.1 $\mathrm{mmol})$ in toluene $(2.0 \mathrm{~mL})$ was added $\mathrm{P}(\mathrm{n}-\mathrm{Bu})_{3}(60.7 \mathrm{mg}, 0.3 \mathrm{mmol})$ at $\mathrm{rt}$. The reaction mixture was stirred until the starting materials disappeared, the solution was directly purified by column chromatography on silica gel (PE/EA =3/1 to $1 / 1$ ) to give the corresponding 8 in $93 \%$ yield $\left(98 \%\right.$ ee) as a white solid. mp: $171-172{ }^{\circ} \mathrm{C},[\alpha]_{0}^{20}=-25.0^{\circ}\left(\mathrm{c}=1.0, \mathrm{CH}_{2} \mathrm{Cl}_{2}\right)$; HPLC condition: chiralpak ODH, $254 \mathrm{~nm}$, $0.5 \mathrm{~mL} / \mathrm{min}$, hexane $/ \mathrm{i}-\mathrm{PrOH}=70 / 30, t_{\text {major }}=11.339 \mathrm{~min}, t_{\text {minor }}=13.096 \mathrm{~min} ;{ }^{1} \mathrm{H} \mathrm{NMR}\left(600 \mathrm{MHz}, \mathrm{CDCl}_{3}\right) \delta 7.31(\mathrm{~s}, 1 \mathrm{H}), 7.15(\mathrm{~d}, J=6.9$ 
$\mathrm{Hz}, 1 \mathrm{H}), 7.01$ (dd, J = 16.5, $9.2 \mathrm{~Hz}, 2 \mathrm{H}), 6.83(\mathrm{~d}, J=5.9 \mathrm{~Hz}, 1 \mathrm{H}), 3.56$ (d, J = $11.5 \mathrm{~Hz}, 3 \mathrm{H}), 3.25$ (s, 3H), $3.19(\mathrm{~d}, J=11.5 \mathrm{~Hz}, 3 \mathrm{H})$, 1.31 (s, 9H) ppm. ${ }^{13} \mathrm{C}$ NMR $\left(151 \mathrm{MHz}, \mathrm{CDCl}_{3}\right) \delta 174.3,153.1,145.2,129.4,123.3,122.4,107.9,79.9,64.2\left({ }^{2} \mathrm{~J}\left({ }^{13} \mathrm{C},{ }^{31} \mathrm{P}\right)=32.5 \mathrm{~Hz}\right)$, $52.9\left({ }^{2} \mathrm{~J}\left({ }^{13} \mathrm{C},{ }^{31} \mathrm{P}\right)=4.2 \mathrm{~Hz}\right), 52.3\left({ }^{2} \mathrm{~J}\left({ }^{13} \mathrm{C},{ }^{31} \mathrm{P}\right)=4.6 \mathrm{~Hz}\right), 28.1,26.6 \mathrm{ppm}$. HRMS (ESI-TOF) calcd. for $\mathrm{C}_{17} \mathrm{H}_{25} \mathrm{~N}_{4} \mathrm{O}_{6} \mathrm{P}[\mathrm{M}+\mathrm{Na}]^{+}$: 435.1409, found: 435.1411 .

(S)-tert-butyl (3-(dimethoxyphosphoryl)-4-(2-(methylamino)phenyl)-5-oxo-4,5-dihydro-1H-pyrazol-4-yl)carbamate 9:

A solution of 8 ( $38.3 \mathrm{mg}, 1.0$ equiv.) in ${ }^{t} \mathrm{BuOH}\left(2.0 \mathrm{~mL}\right.$ ) was added ${ }^{t} \mathrm{BuOK}$ (10.4 mg, 1.0 equiv.) at rt. The reaction mixture was stirred until the starting materials disappeared. The mixture was diluted with $\mathrm{H}_{2} \mathrm{O}$ and extracted with $\mathrm{CH}_{2} \mathrm{Cl}_{2}$ twice. The combined organic extracts were dried over $\mathrm{Na}_{2} \mathrm{SO}_{4}$ and the filtrate was concentrated in vacuo. The resulting residue was purified by column chromatography on silica gel (PE/EA $=1 / 2$ to $1 / 5)$ to give the product in $88 \%$ yield as a white solid $\left(96: 4 d r, 97 \%\right.$ ee). $\mathrm{mp}: 183-184{ }^{\circ} \mathrm{C}$, $[\alpha]_{0}^{20}=-26.4^{\circ}\left(\mathrm{C}=1.0, \mathrm{CH}_{2} \mathrm{Cl}_{2}\right)$; HPLC condition: chiralpak IB, $254 \mathrm{~nm}, 0.5 \mathrm{~mL} / \mathrm{min}$, hexane/i-PrOH $=70 / 30, t_{\text {major }}=14.217 \mathrm{~min}, t_{\mathrm{minor}}=$ $23.021 \mathrm{~min} ;{ }^{1} \mathrm{H}$ NMR $\left(600 \mathrm{MHz}, \mathrm{CDCl}_{3}\right) \delta 8.66(\mathrm{~d}, J=90.1 \mathrm{~Hz}, 1 \mathrm{H}), 7.31(\mathrm{t}, J=7.4 \mathrm{~Hz}, 1 \mathrm{H}), 7.14(\mathrm{~d}, J=7.2 \mathrm{~Hz}, 1 \mathrm{H}), 7.05(\mathrm{~d}, J=6.8$ $\mathrm{Hz}, 1 \mathrm{H}), 6.84(\mathrm{~d}, J=6.9 \mathrm{~Hz}, 1 \mathrm{H}), 6.72(\mathrm{~s}, 1 \mathrm{H}), 6.37$ (s, 1H), $3.73(\mathrm{dd}, J=11.4,4.3 \mathrm{~Hz}, 6 \mathrm{H}), 3.23(\mathrm{~s}, 3 \mathrm{H}), 1.19(\mathrm{~d}, J=174.7 \mathrm{~Hz}, 9 \mathrm{H})$. ${ }^{13} \mathrm{C}$ NMR $\left(151 \mathrm{MHz}, \mathrm{CDCl}_{3}\right) \delta 173.4,153.3,143.6,129.7,123.4,123.1,108.6,80.4,63.8,53.9\left({ }^{2} \mathrm{~J}\left({ }^{13} \mathrm{C},{ }^{31} \mathrm{P}\right)=5.5 \mathrm{~Hz}\right), 53.8\left({ }^{2} J\left({ }^{13} \mathrm{C}\right.\right.$,

$\left.{ }^{31} \mathrm{P}\right)=5.5 \mathrm{~Hz}$ ), 28.0, 26.8. HRMS (ESI-TOF) calcd. for $\mathrm{C}_{17} \mathrm{H}_{25} \mathrm{~N}_{4} \mathrm{O}_{6} \mathrm{P}[\mathrm{M}+\mathrm{Na}]^{+}:$435.1409, found: 435.1420 .

\section{X-Ray crystallographic analysis of 4 aaa and determination of the absolute configurations}

\begin{tabular}{|c|c|}
\hline Empirical formula & $\mathrm{C}_{17} \mathrm{H}_{23} \mathrm{~N}_{4} \mathrm{O}_{6} \mathrm{P}$ \\
\hline Formula weight & 410.36 \\
\hline Temperature/K & $279.66(10)$ \\
\hline Crystal system & orthorhombic \\
\hline Space group & $\mathrm{P} 2{ }_{1} 2_{1} 2_{1}$ \\
\hline $\mathrm{a} / \AA ̊$ & $9.73106(13)$ \\
\hline $\mathrm{b} / \AA ̊$ & 10.22183(14) \\
\hline$c / \AA ̊$ & $19.6704(2)$ \\
\hline$\alpha /^{\circ}$ & 90 \\
\hline$\beta /^{\circ}$ & 90 \\
\hline $\mathrm{Y}^{\circ}$ & 90 \\
\hline Volume $/ \AA^{3}$ & $1956.60(4)$ \\
\hline Z & 4 \\
\hline$\rho_{\text {calc }} \mathrm{g} / \mathrm{cm}^{3}$ & 1.393 \\
\hline$\mu / \mathrm{mm}^{-1}$ & 1.623 \\
\hline$F(000)$ & 864.0 \\
\hline Crystal size $/ \mathrm{mm}^{3}$ & $0.13 \times 0.12 \times 0.12$ \\
\hline
\end{tabular}




\begin{tabular}{|l|l|}
\hline Radiation & CuKa $(\lambda=1.54184)$ \\
\hline $2 \Theta$ range for data collection/ & 8.992 to 143.014 \\
\hline Index ranges & $-11 \leq \mathrm{h} \leq 11,-12 \leq \mathrm{k} \leq 8,-24 \leq \mathrm{I} \leq 23$ \\
\hline Reflections collected & 10421 \\
\hline Independent reflections & $3449\left[\mathrm{R}_{\text {int }}=0.0281, \mathrm{R}_{\text {sigma }}=0.0270\right]$ \\
\hline Data/restraints/parameters & $3449 / 26 / 320$ \\
\hline Goodness-of-fit on $\mathrm{F}^{2}$ & 1.050 \\
\hline Final $\mathrm{R}$ indexes [l>=2 $\sigma(\mathrm{I})]$ & $\mathrm{R}_{1}=0.0539, \mathrm{wR}_{2}=0.1328$ \\
\hline Final $\mathrm{R}$ indexes [all data] & $\mathrm{R}_{1}=0.0561, \mathrm{wR}_{2}=0.1348$ \\
\hline Largest diff. peak/hole / e $\AA^{-3}$ & $0.92 /-0.47$ \\
\hline Flack parameter & $0.030(14)$ \\
\hline
\end{tabular}

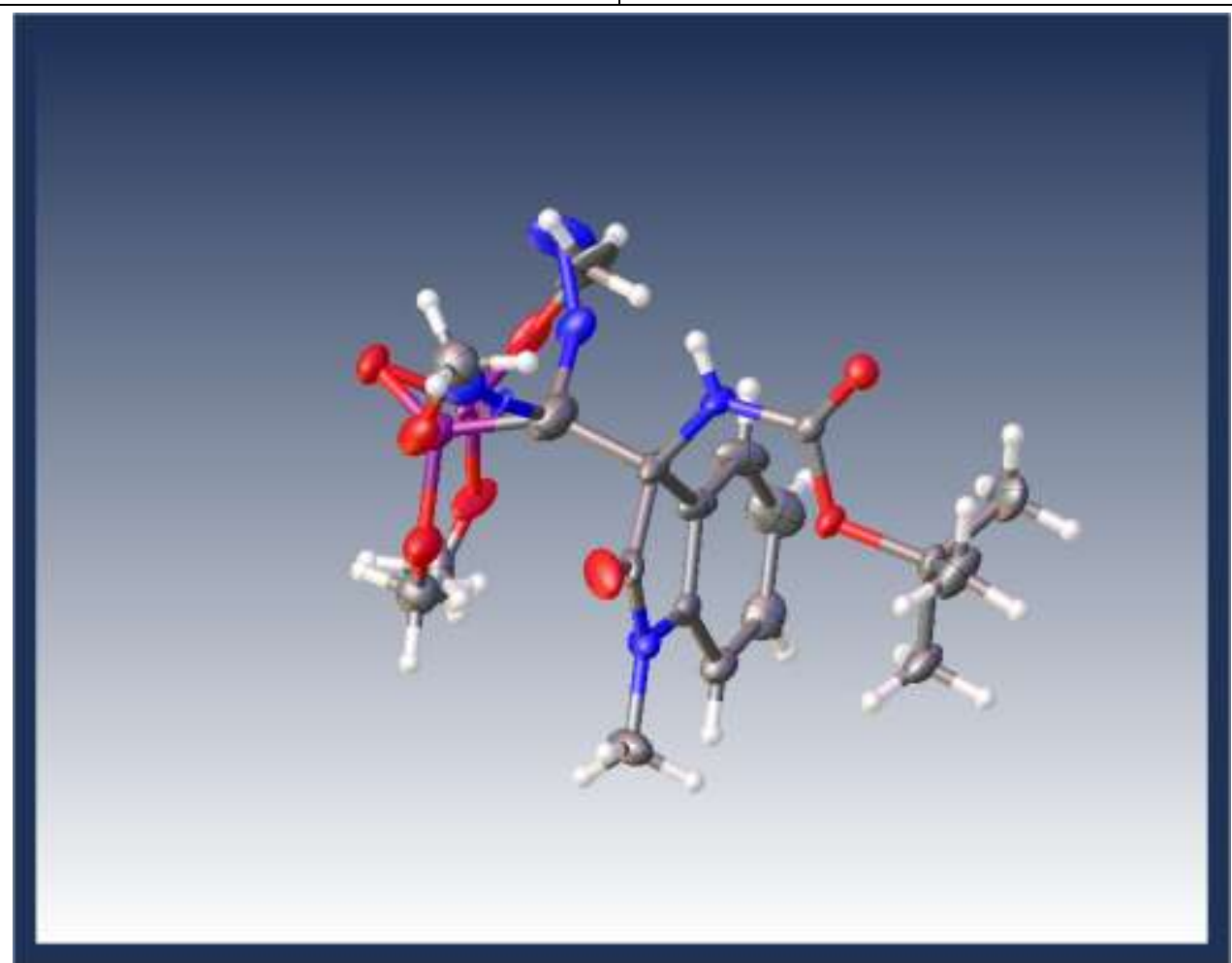




\section{Proposed Mechanism}

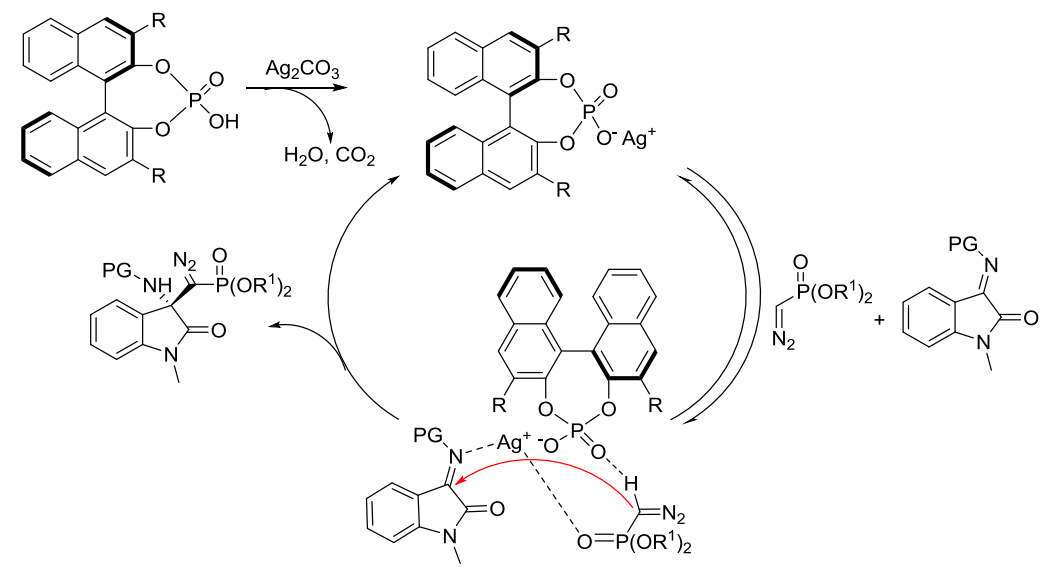

Based on the stereocontrolled results and precedent examples ${ }^{7}$, the proposed mechanism was shown as above. The chiral silver phosphate acts as bifunctional catalyst, the silver (I) ion as Lewis acid activates both the imine and the diazo phosphonate by simultaneously coordination with them, and then the negative carbon of the diazo phosphonate nucleophile addition the imine from its

Re face. The phosphoryl oxygen would function as a Brønsted basic site and assist to deprotonate the $\alpha$ - $\mathrm{H}$ of diazo phosphonate to give the desired Mannich products. 


\section{References}

[1] Yan, W.; Wang, D.; Feng, J.; Li, P.; Zhao, D.; Wang, R. Org. Lett. 2012, 14, 2512.

[2] Du, T.; Du, F.; Ning, Y.; Peng, Y. Org. Lett. 2015, 17, 1308.

[3] (a) Romanov-Michailidis, F.; Guenee, L.; Alexakis, A. Angew. Chem., Int. Ed. 2013, 125, 9436. (b) Pousse, G.; Devineau, A.; Dalla, V.; Humphreys, L.; Lasne, M.-C.; Rouden, J.; Blanchet, J. Tetrahedron. 2009, 65, 10617.

[4] Cheng, X.; Goddard, R.; Buth, G.; List, B. Angew. Chem. Int. Ed. 2008, 47, 5079.

[5] Hashimoto, T.; Maruoka, K. J. Am. Chem. Soc ., 2007, 129, 10054.

[6] (a) Yasui, E.; Wada, M.; Takamura, N. Chemical and Pharmaceutical Bulletin. 2007, 55, 1652. (b) Metwally, S. A. M.; Abdel Moneim,M. I.; Elossely, Y. A.; Awad, R. I.; Abou-Hadeed, K. Chem. Het. Comp. 2010, 46, 426.

[7] Yamamoto, H.; Futatsugi, K. Angew. Chem., Int. Ed. 2005, 44, 1924. 
11. NMR spectra for chiral phosphoric acids and products
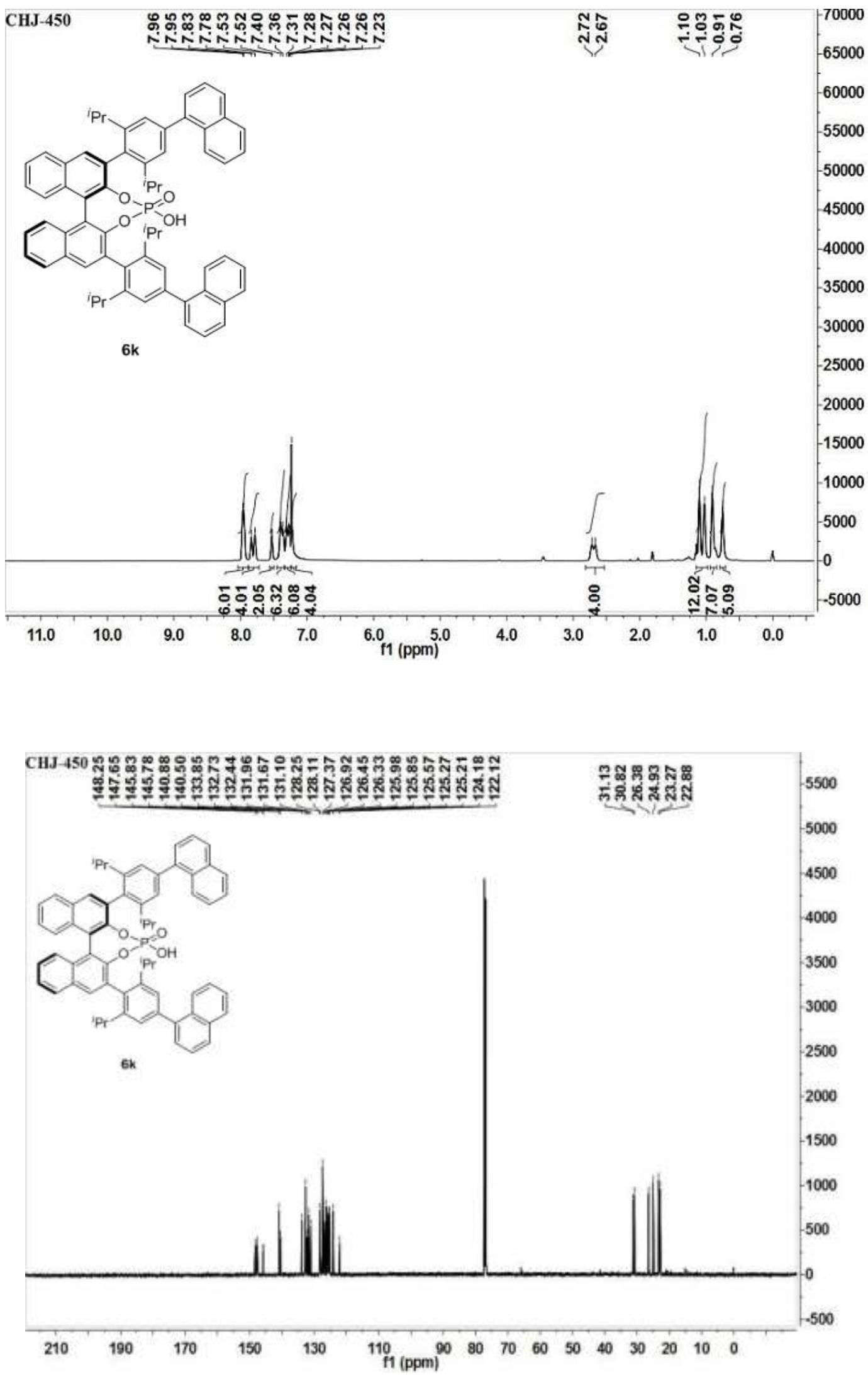

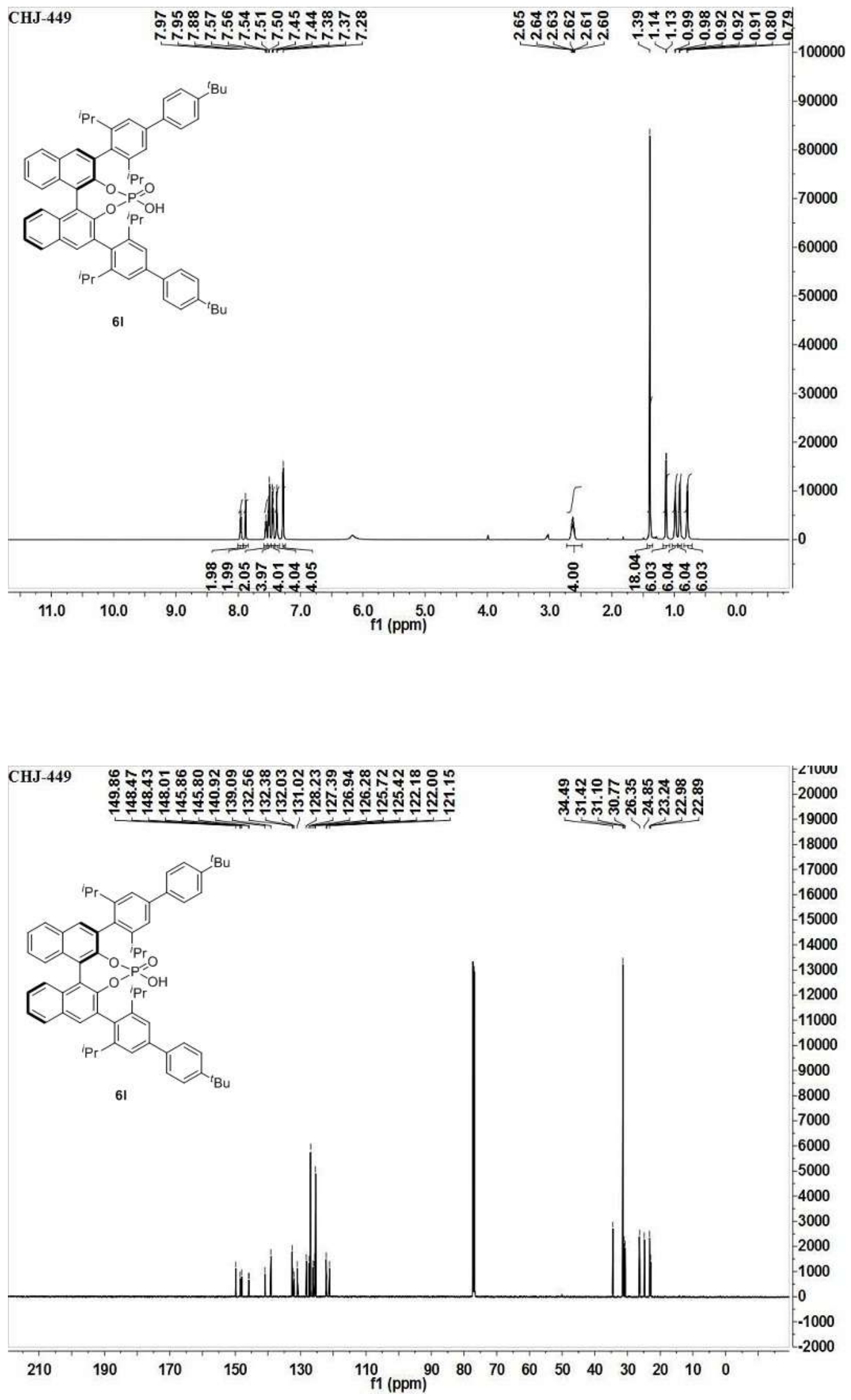

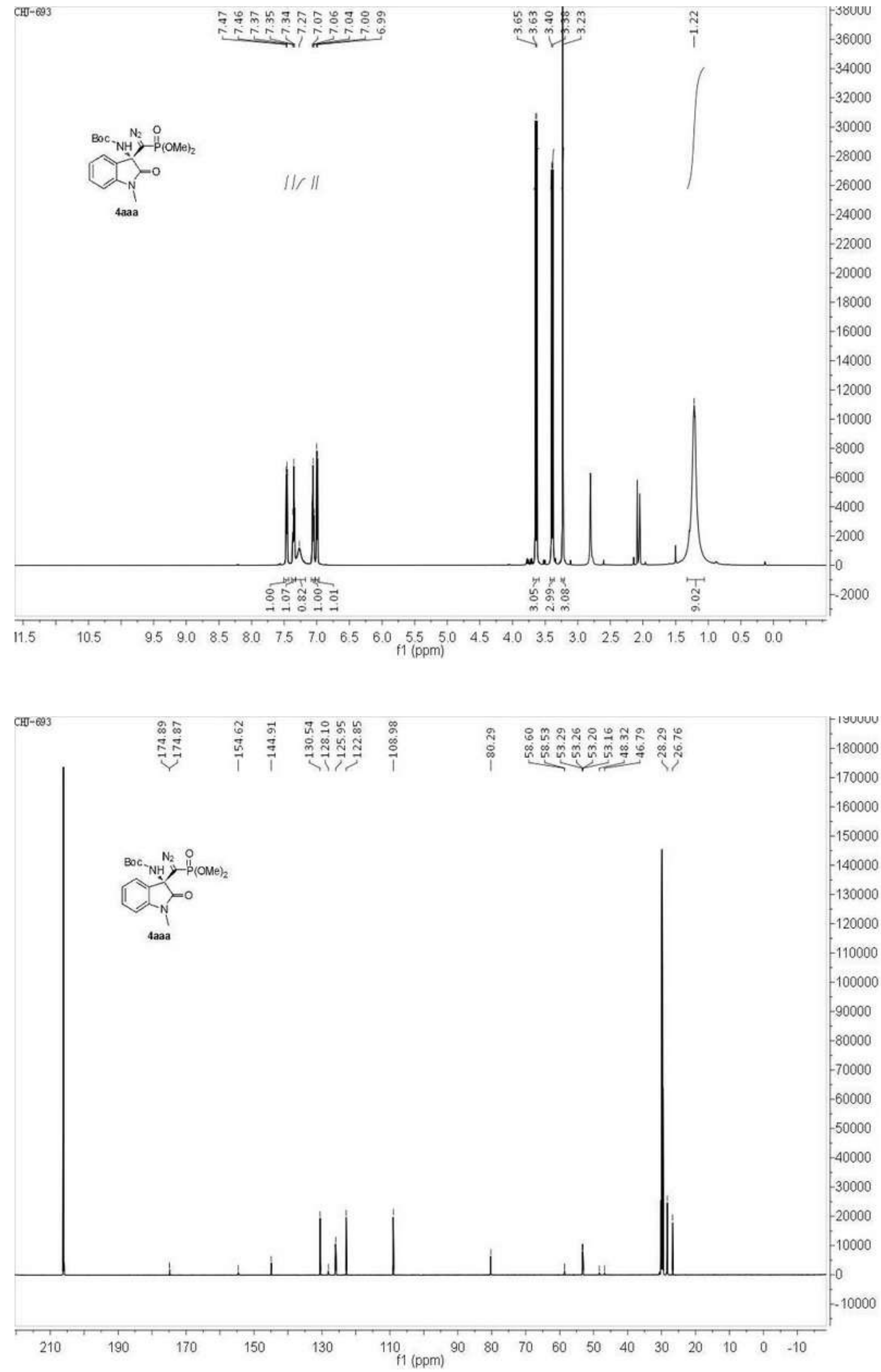

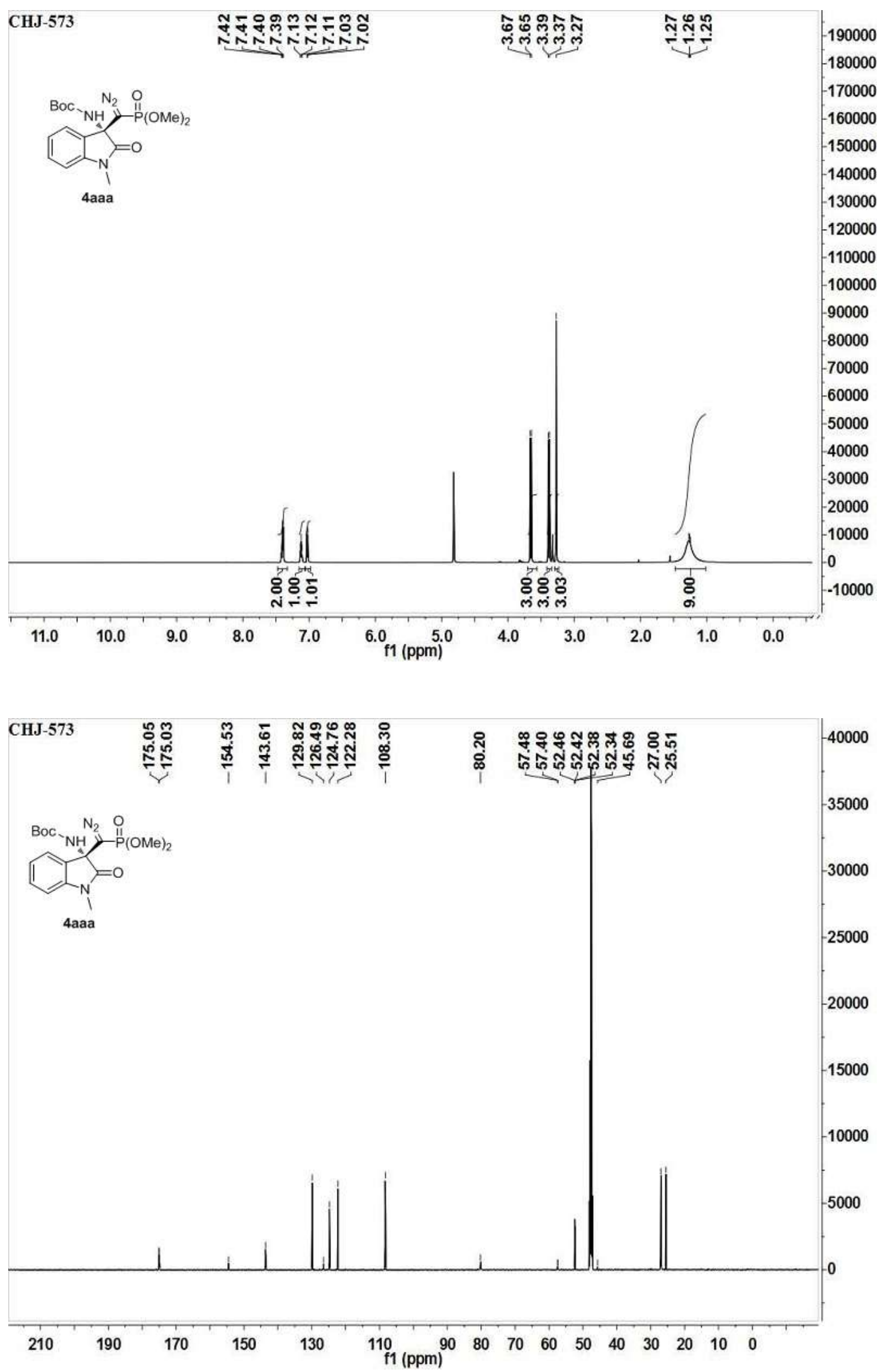

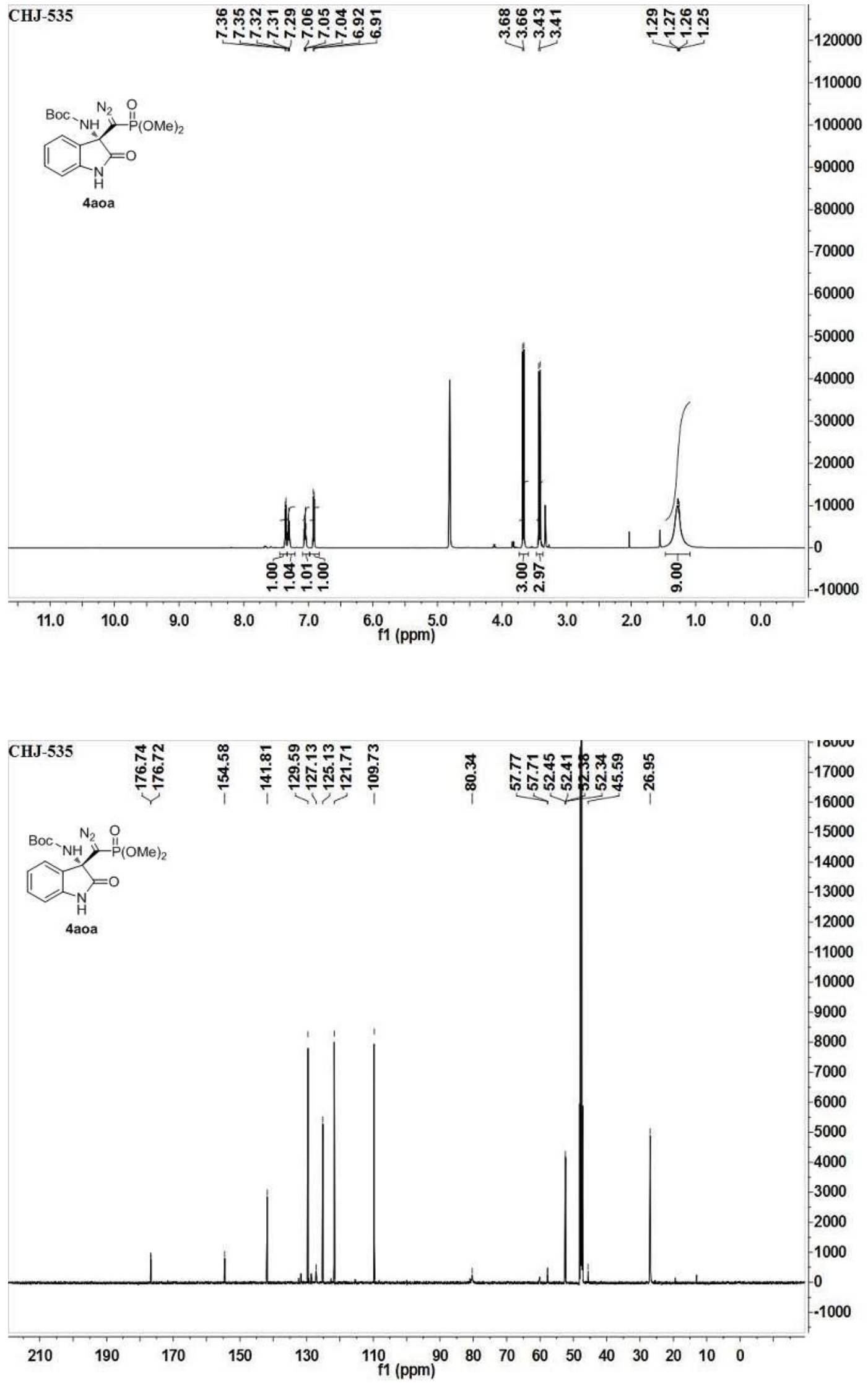

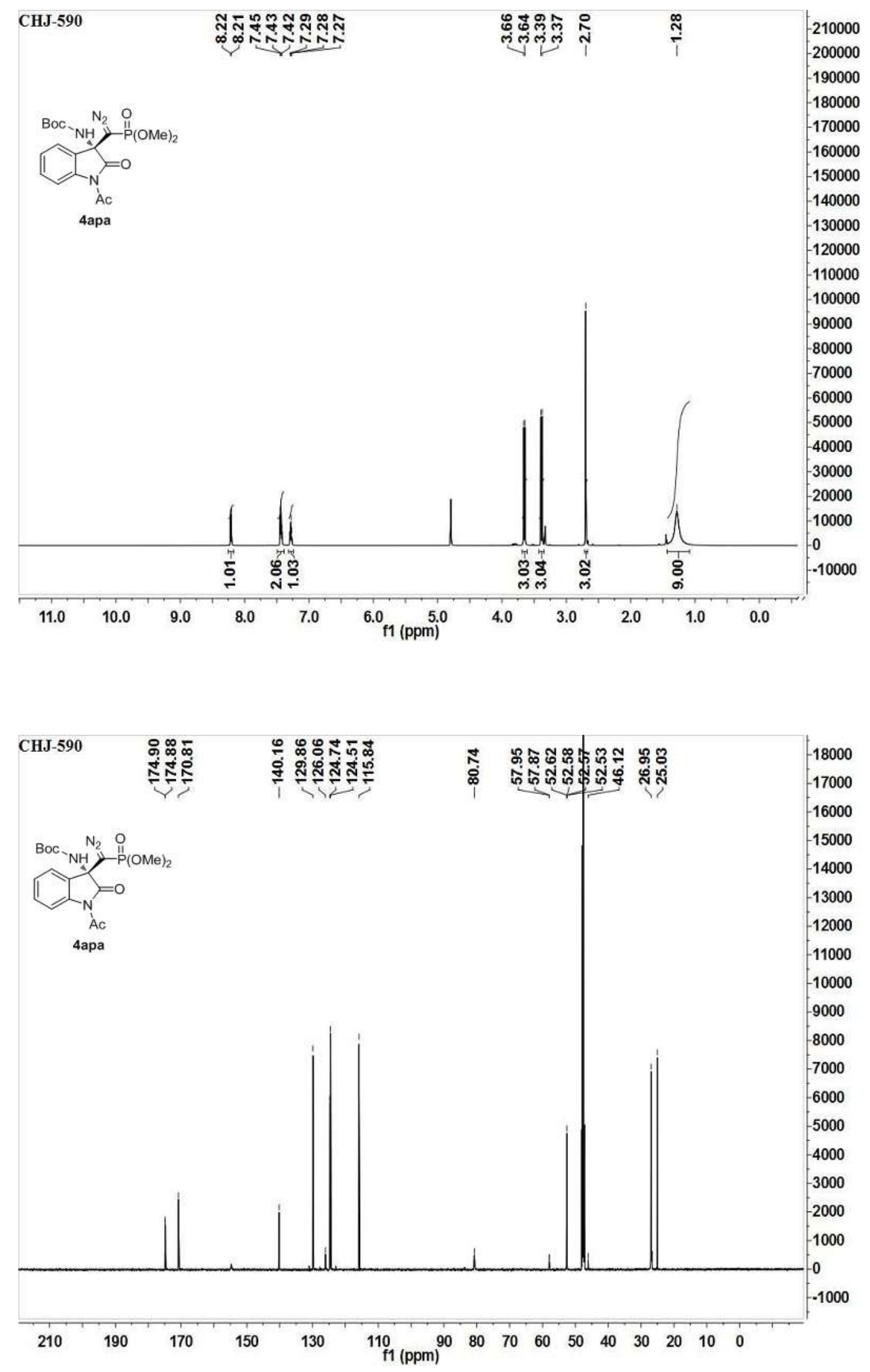

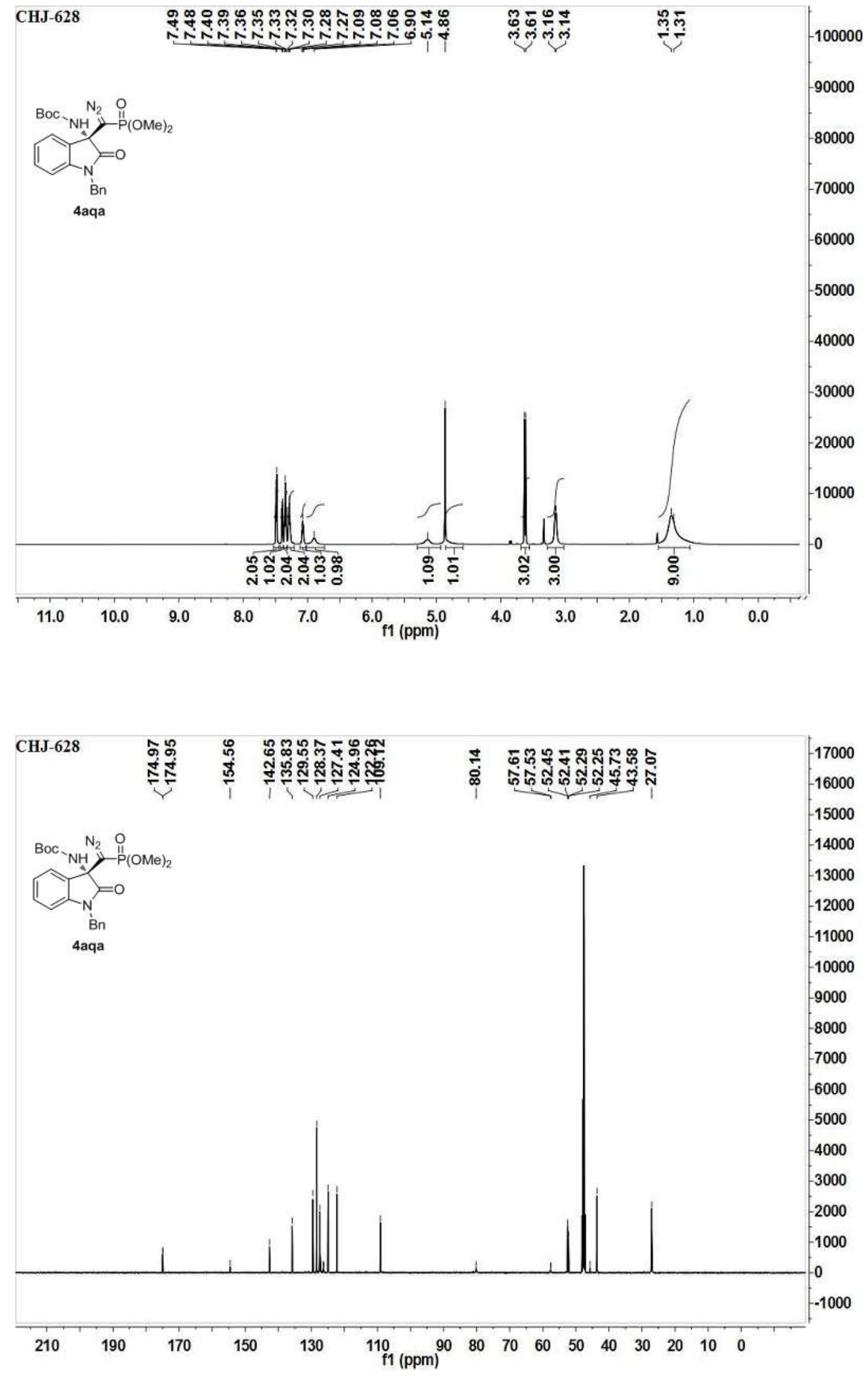

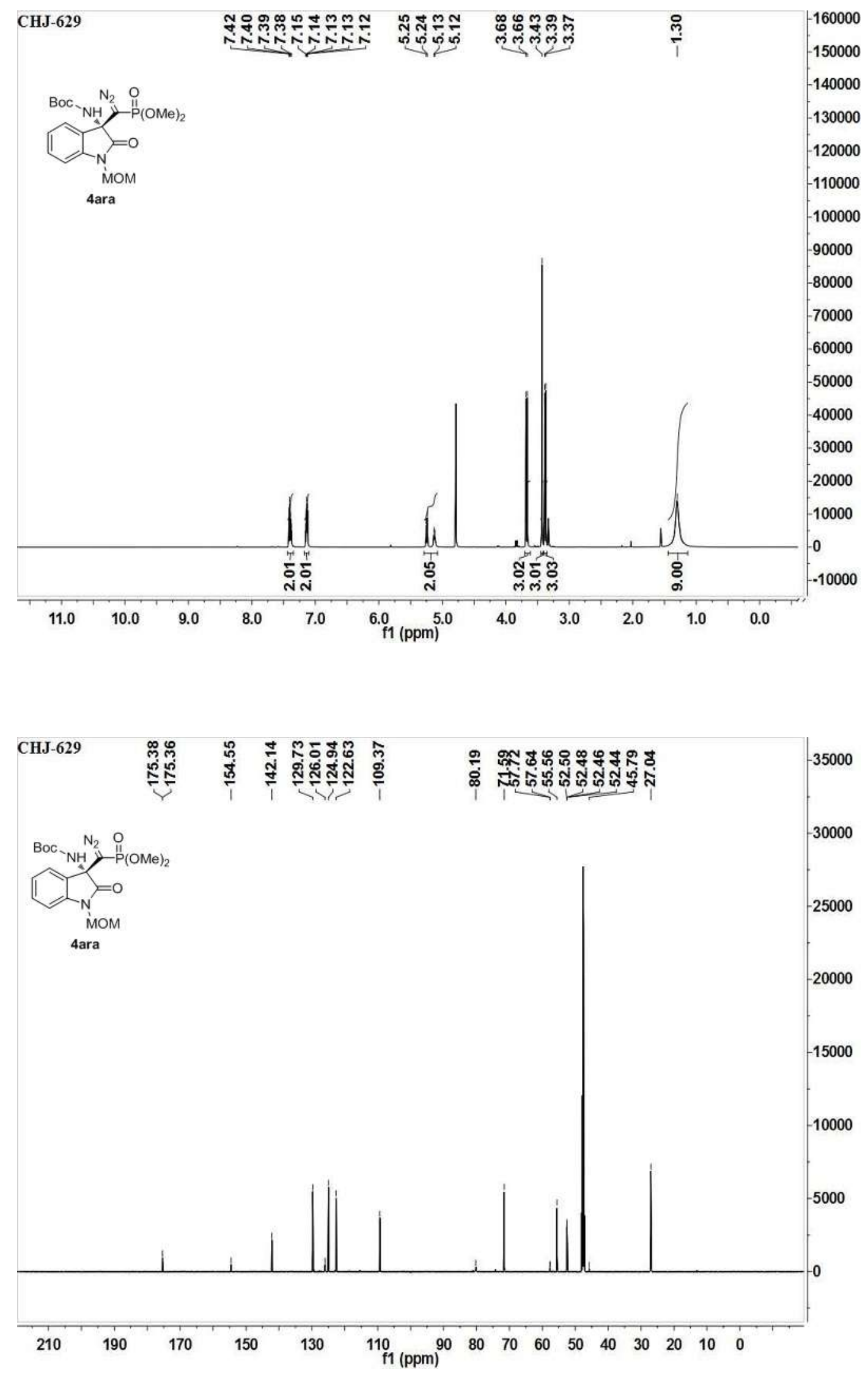

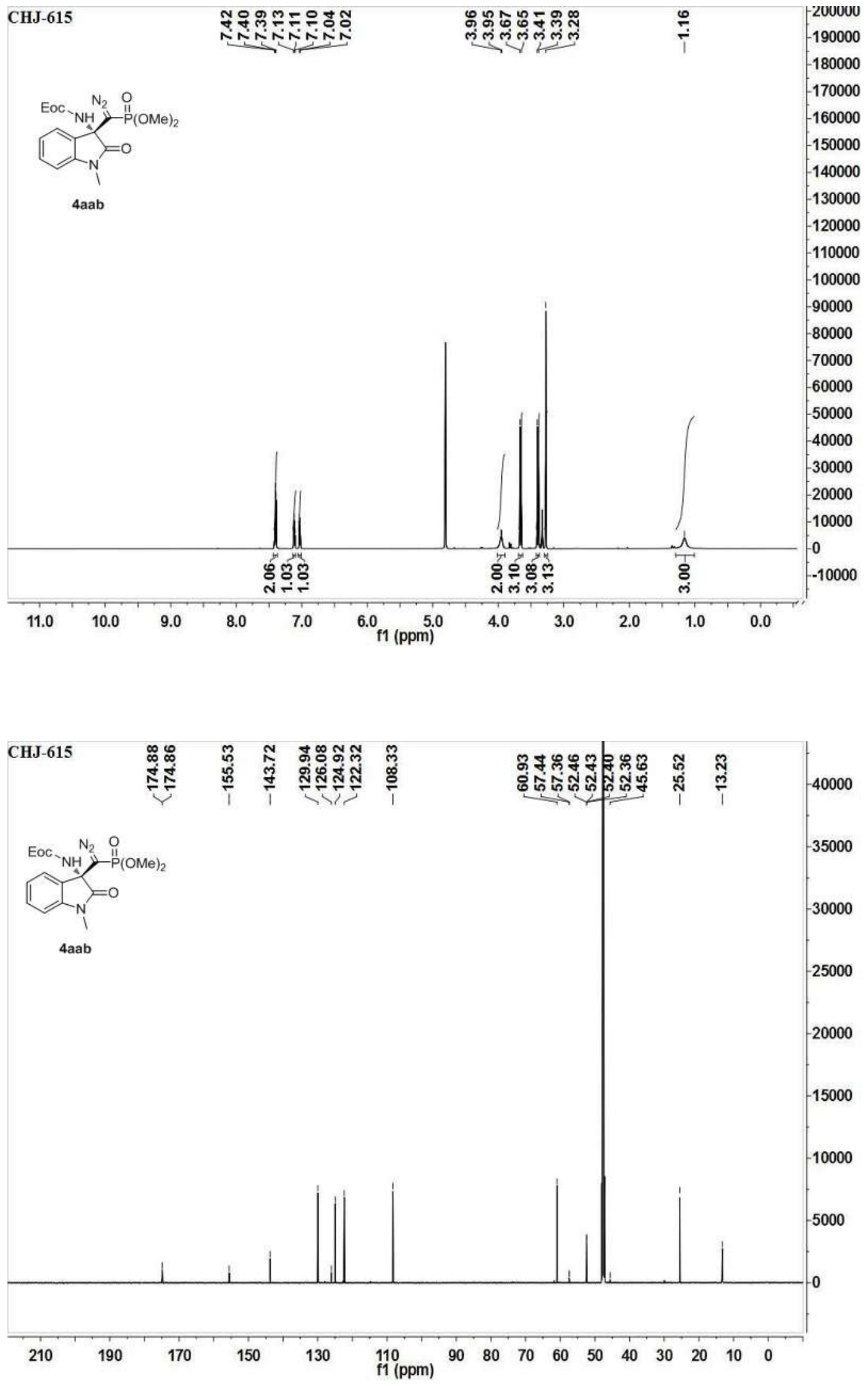

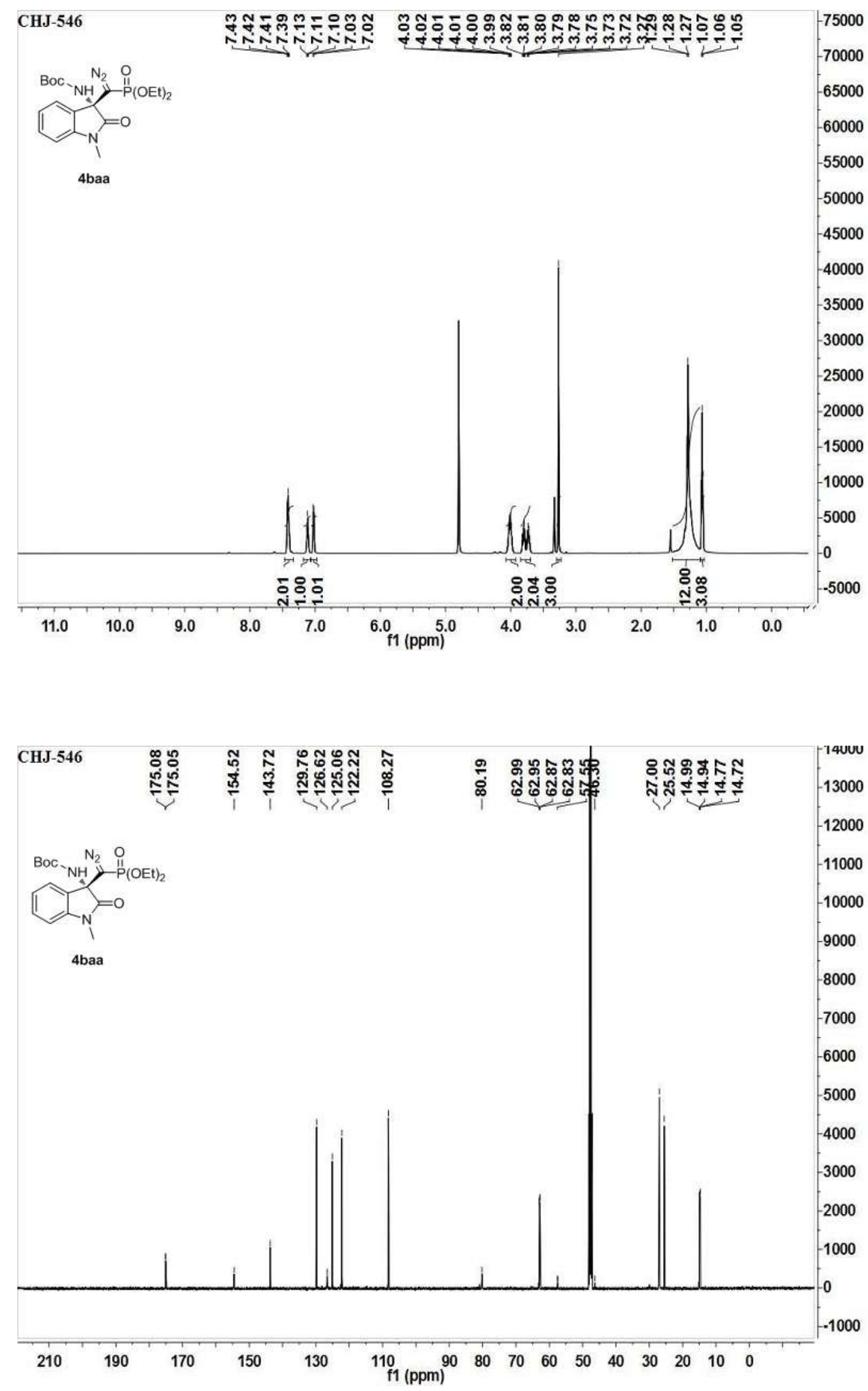

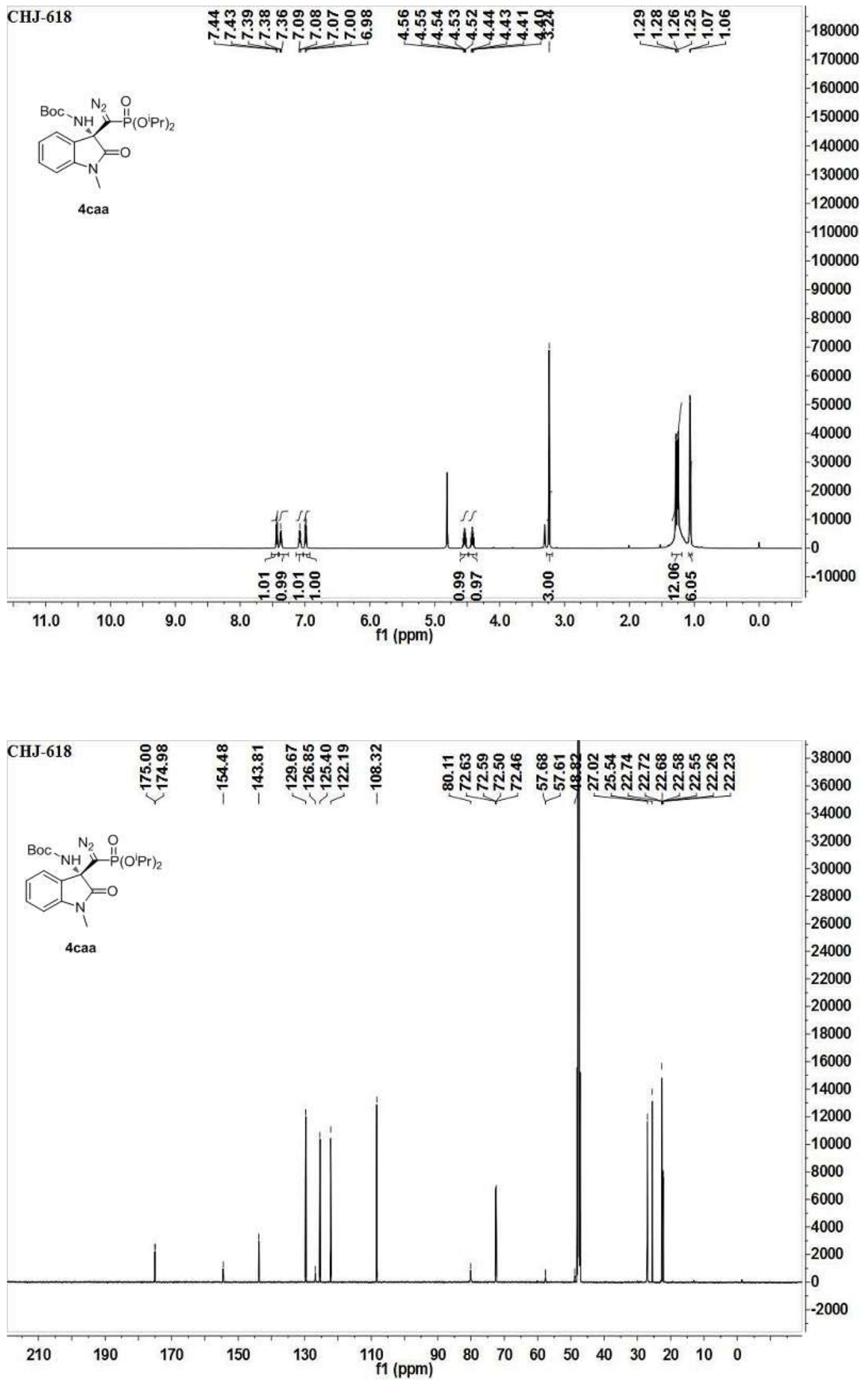

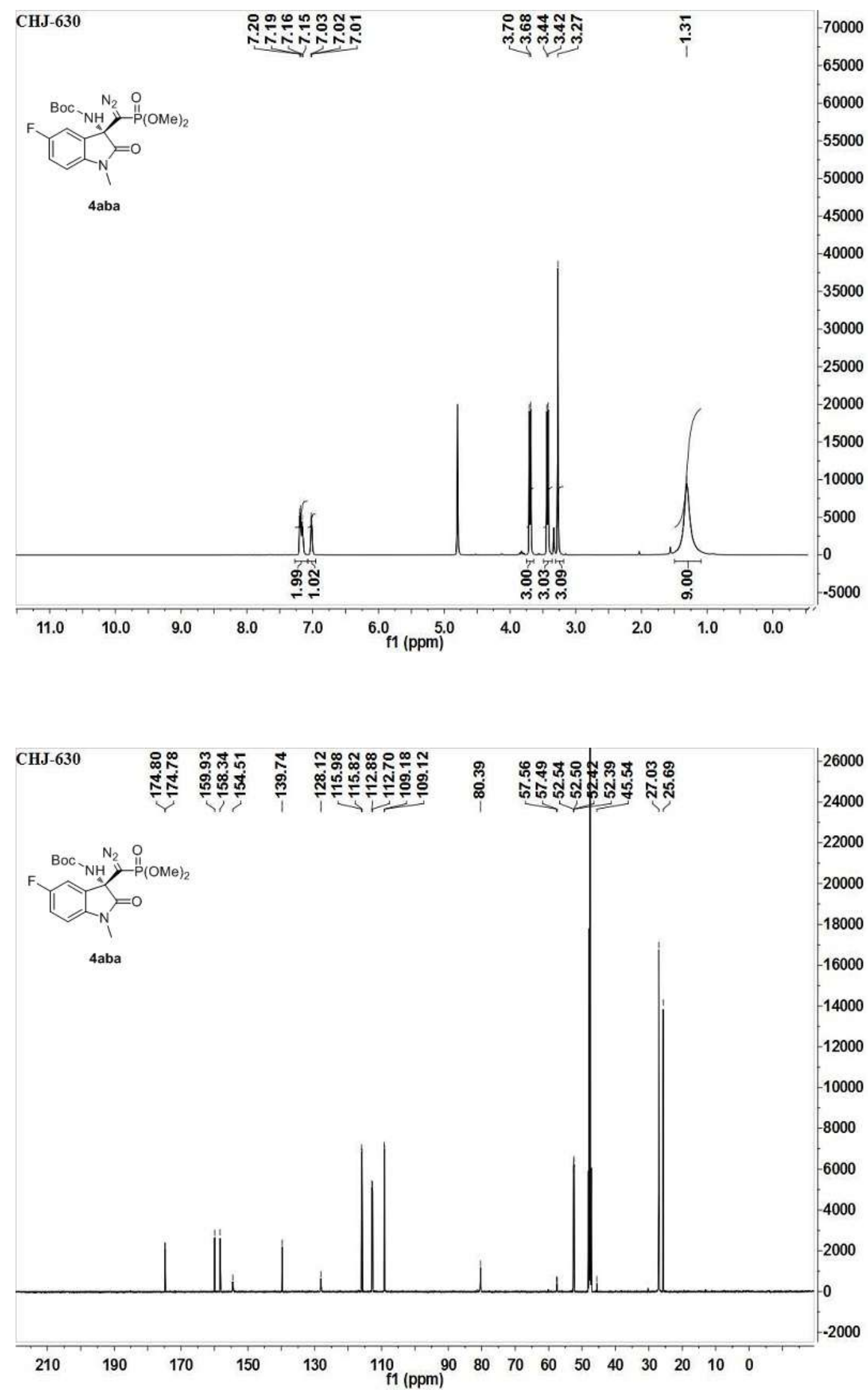

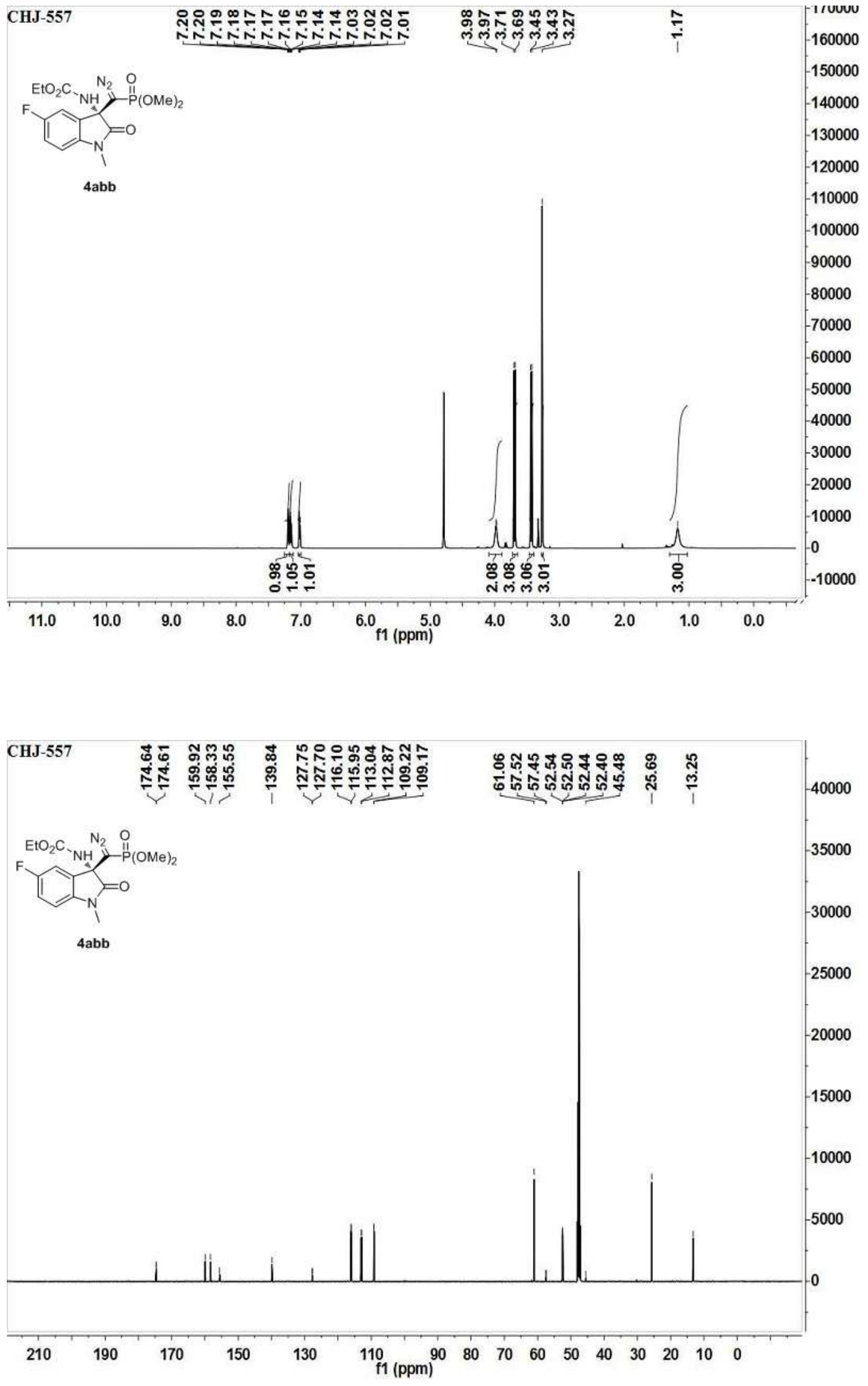

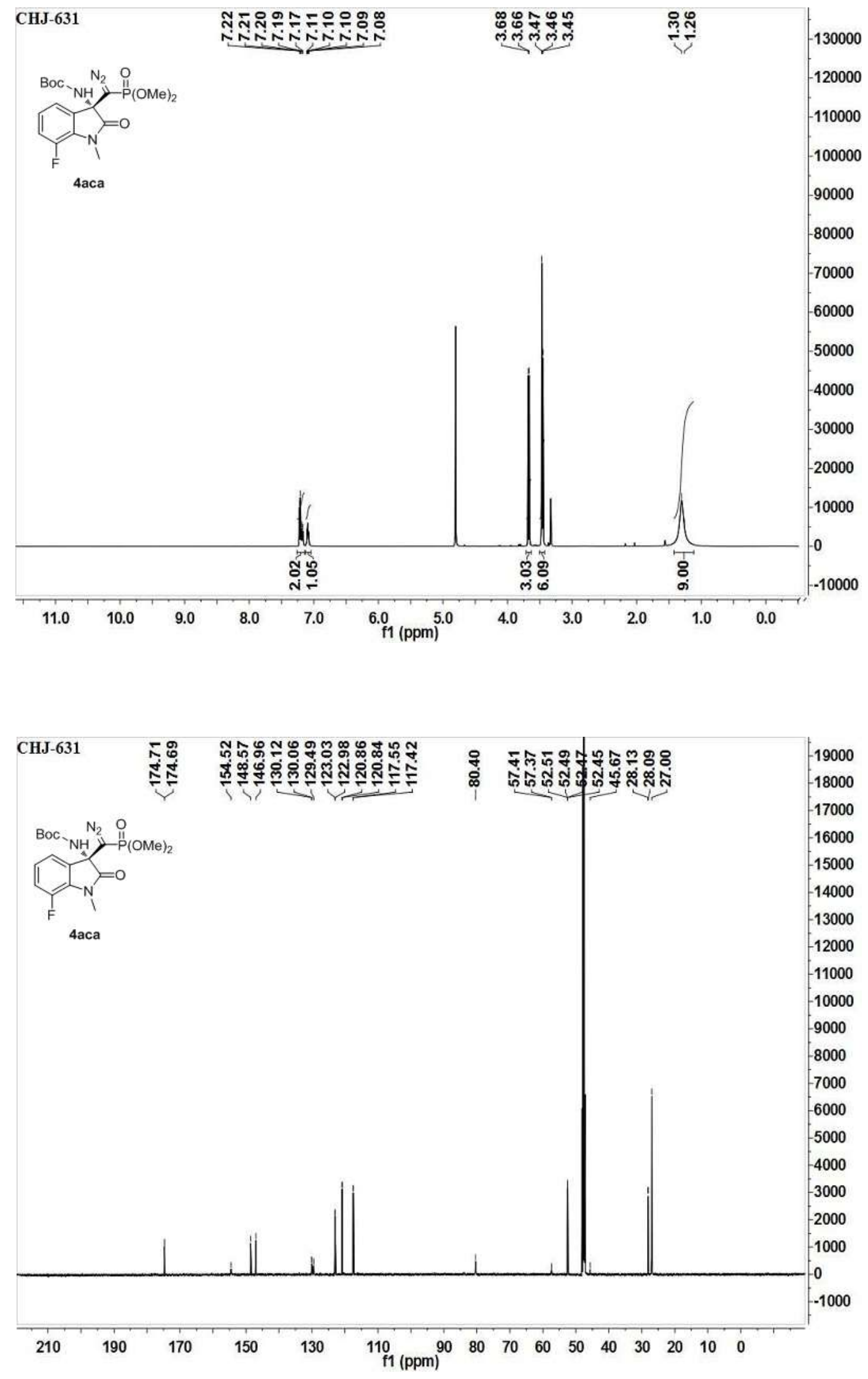

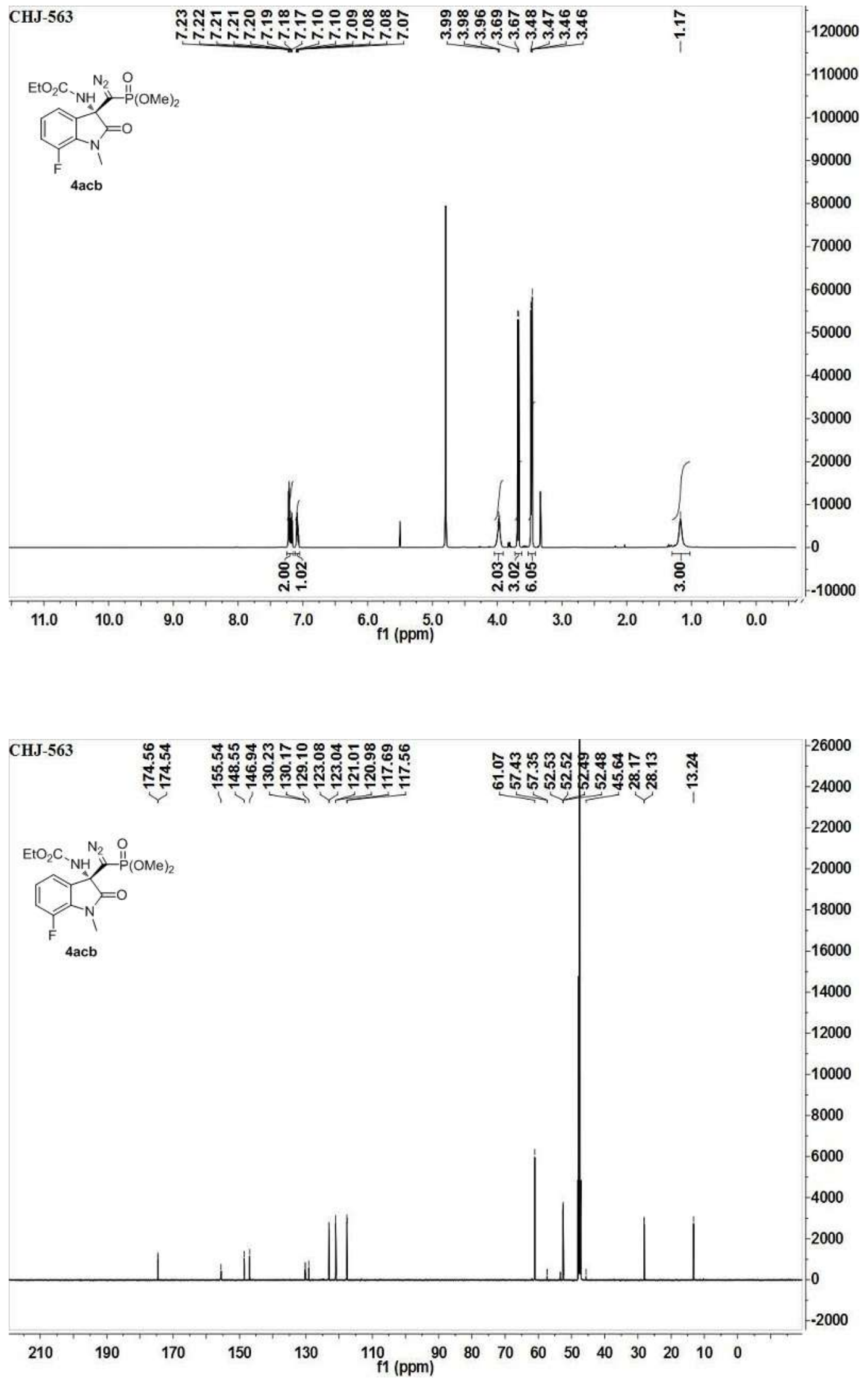

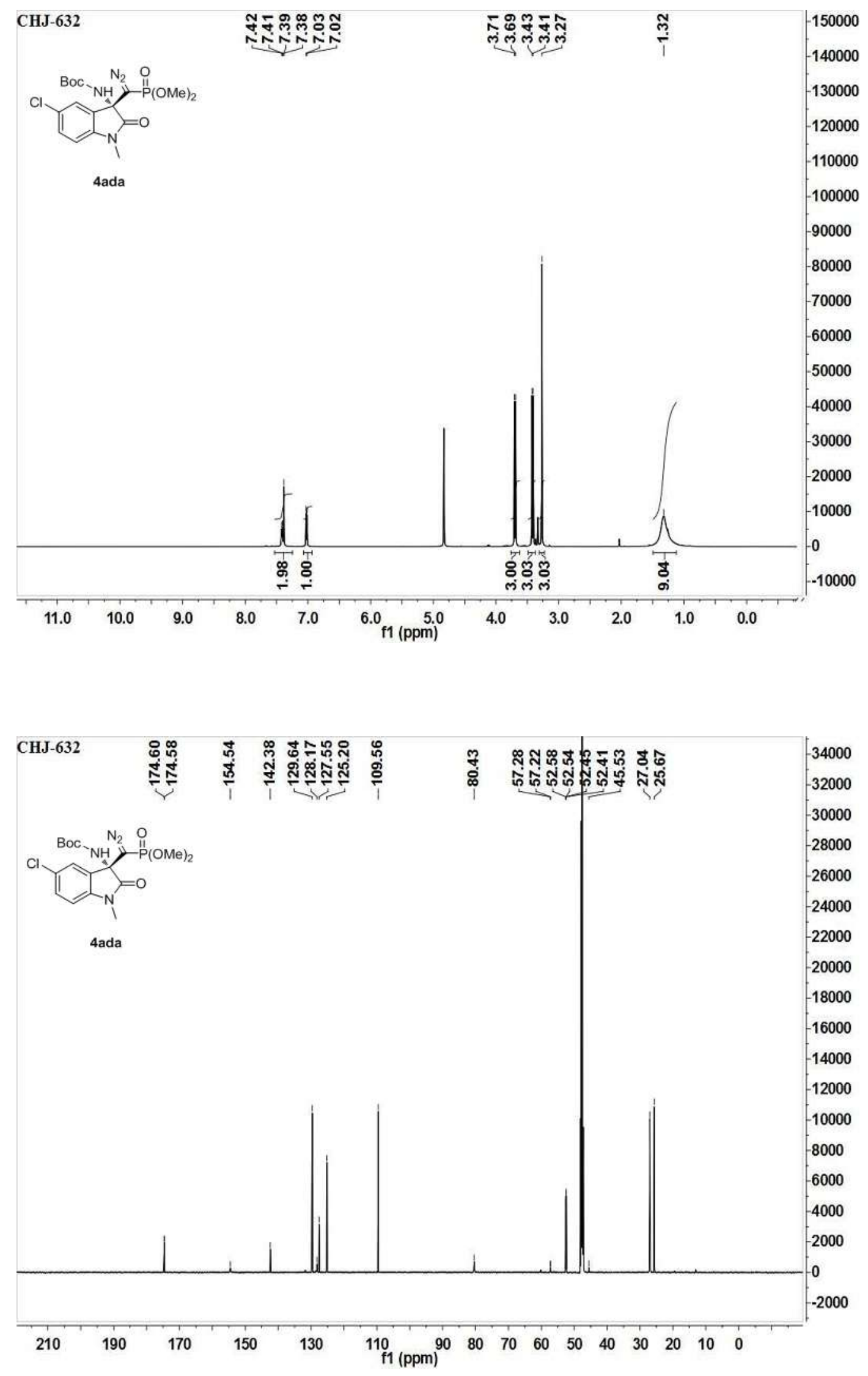

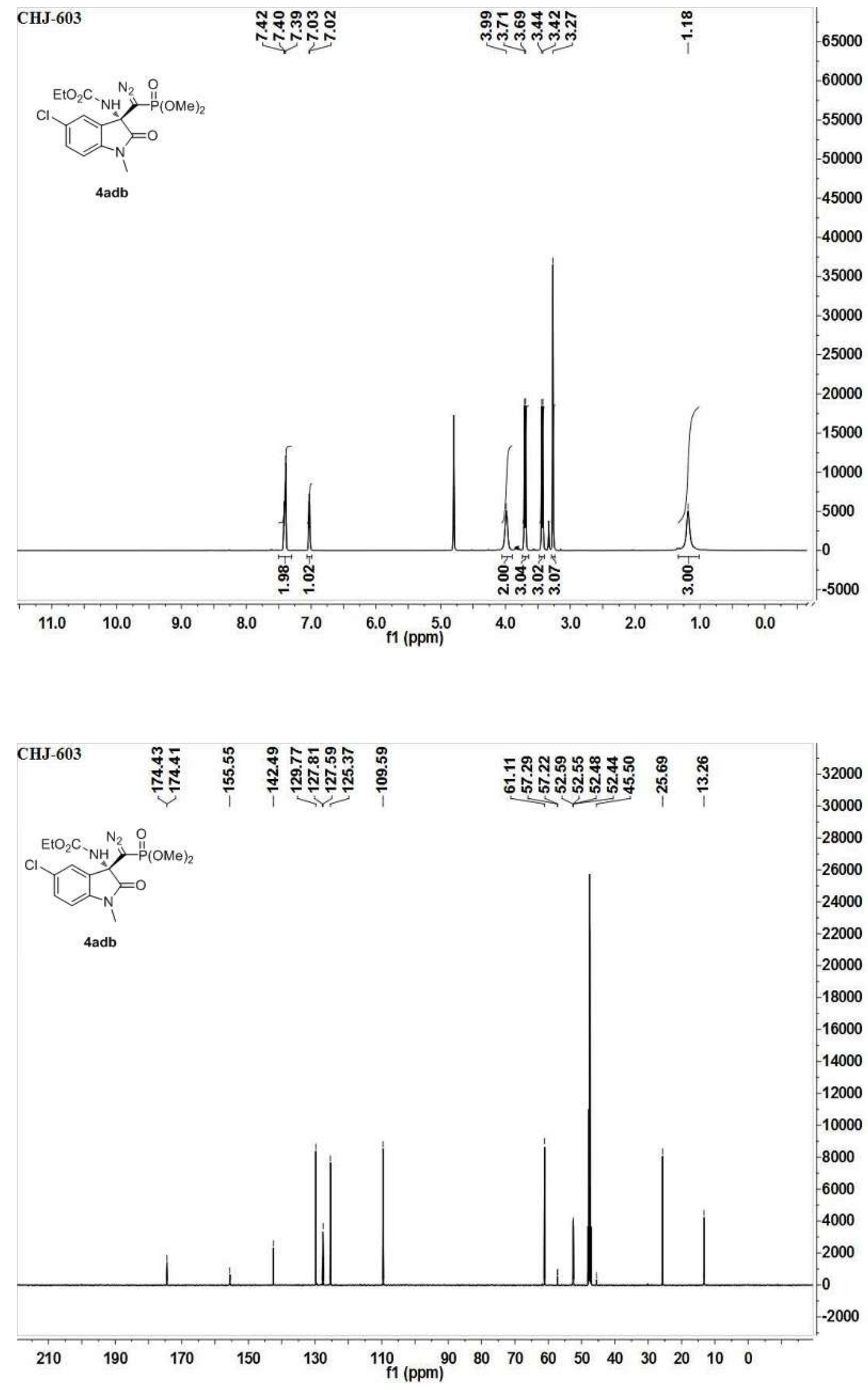

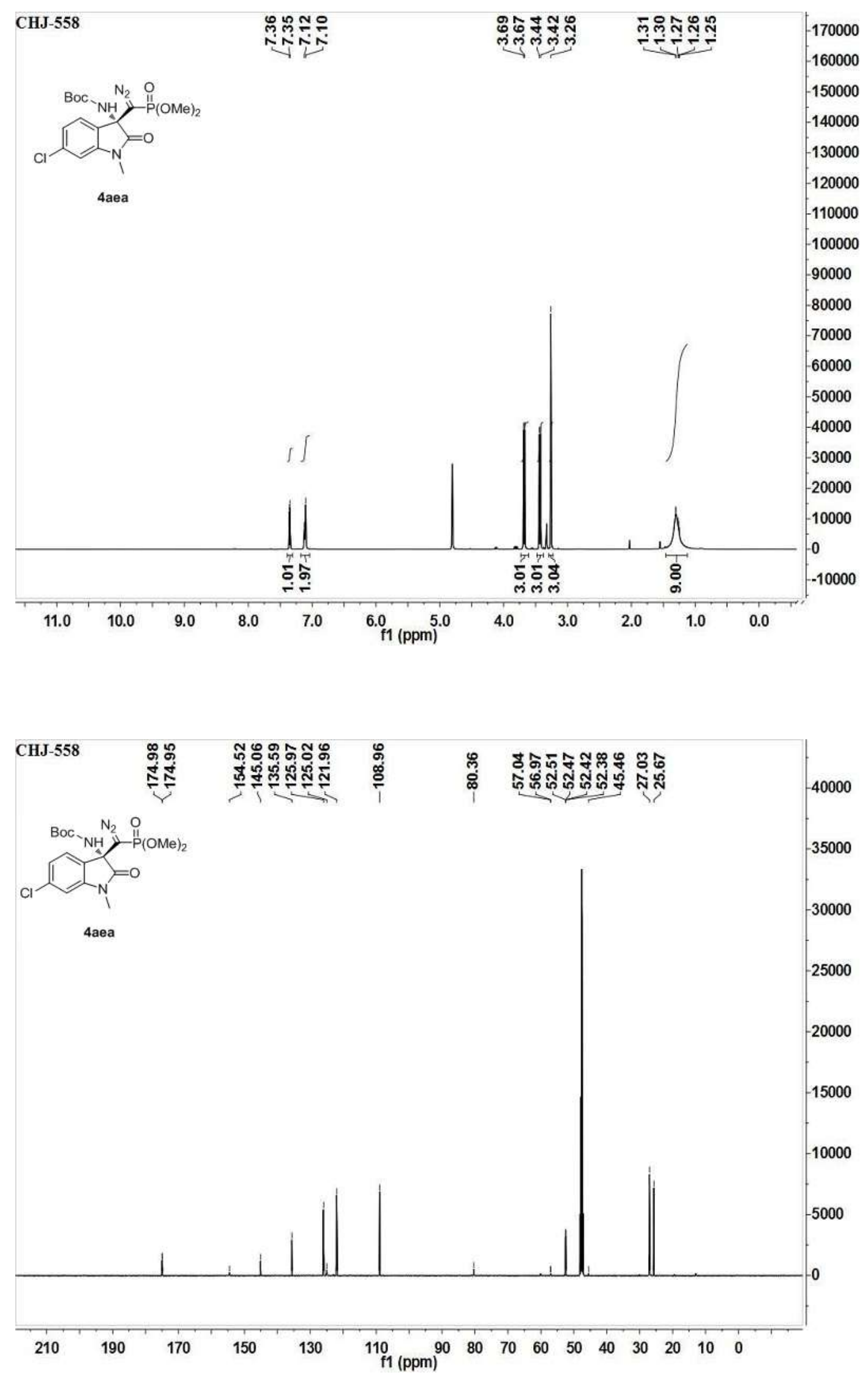

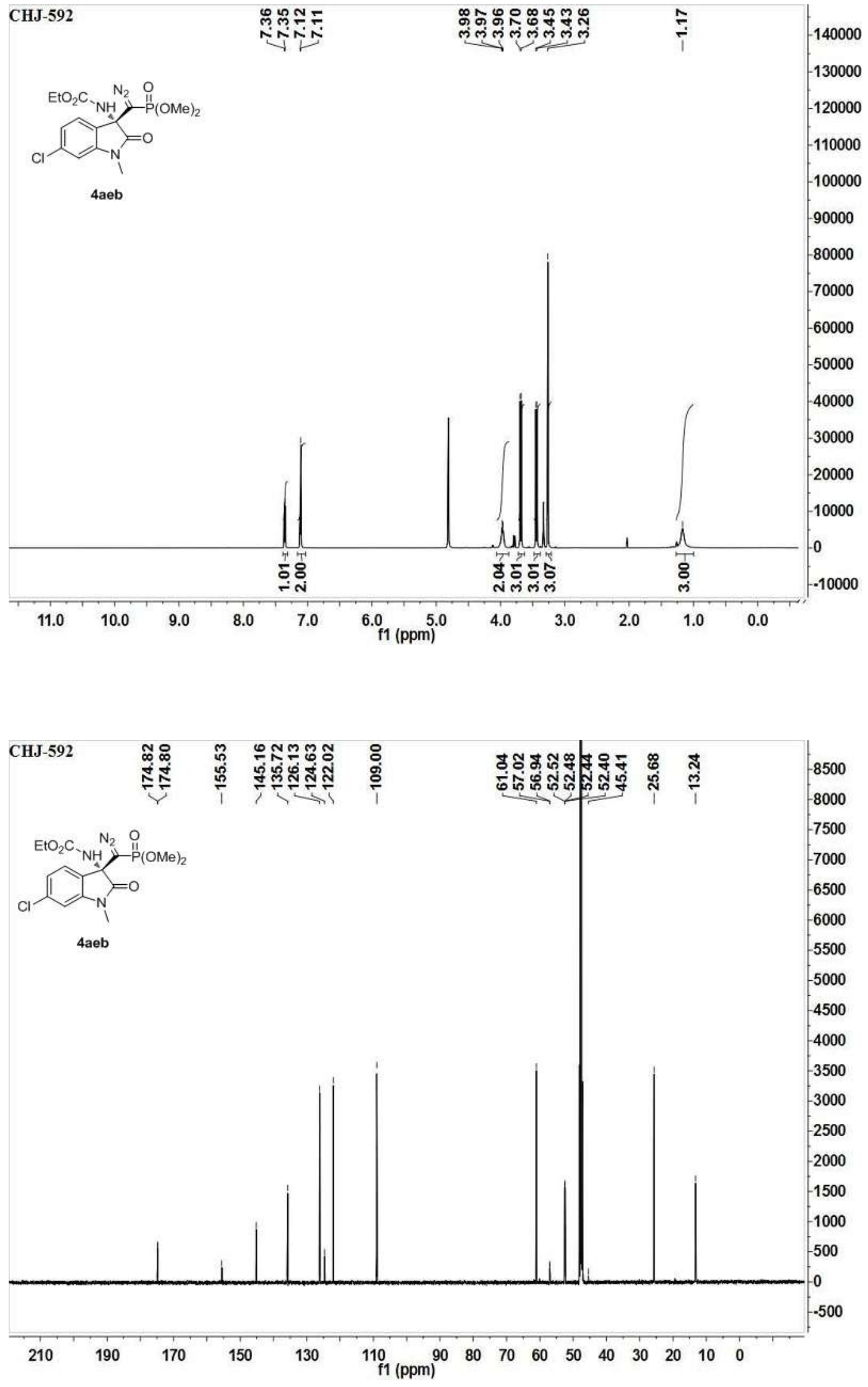

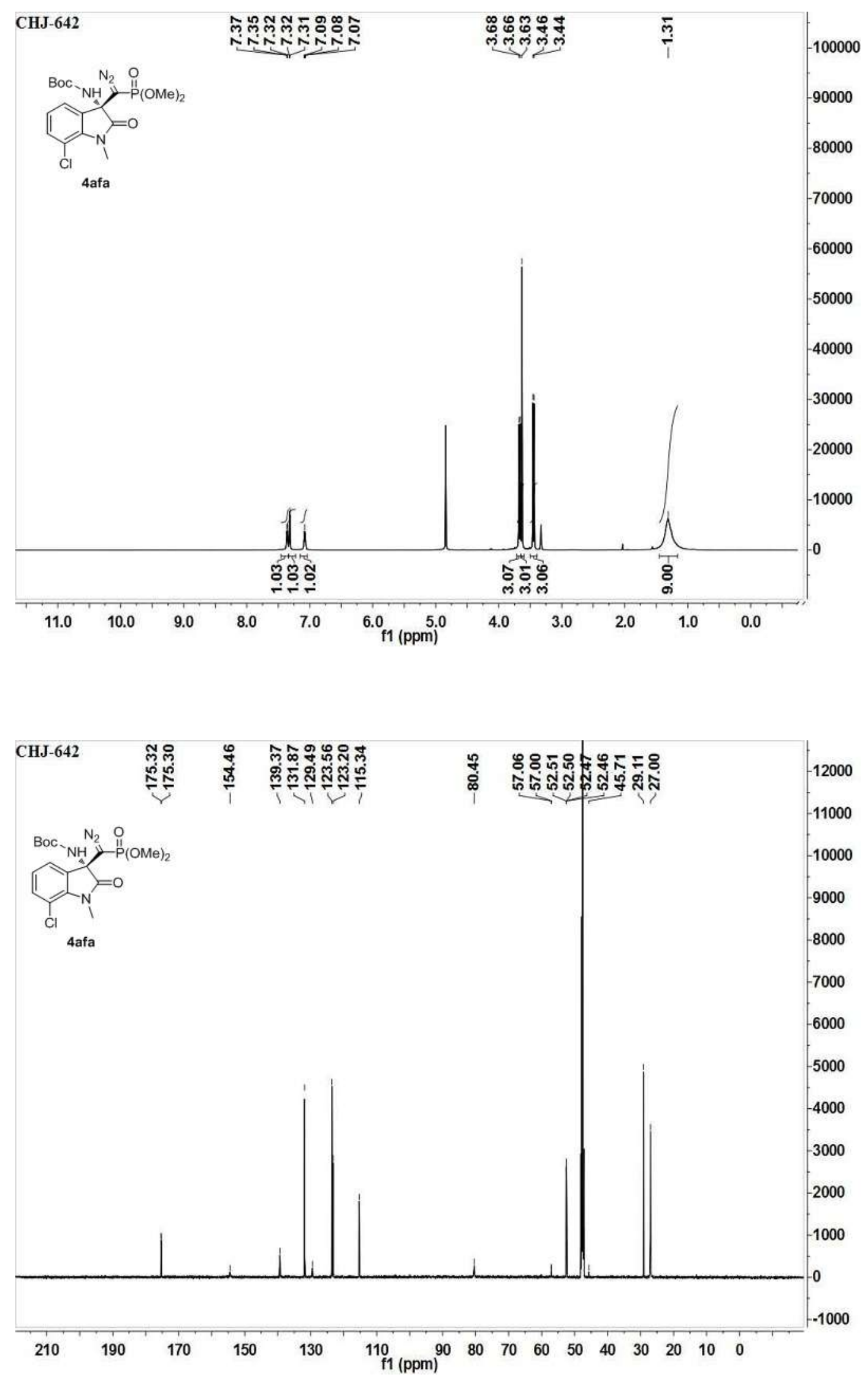

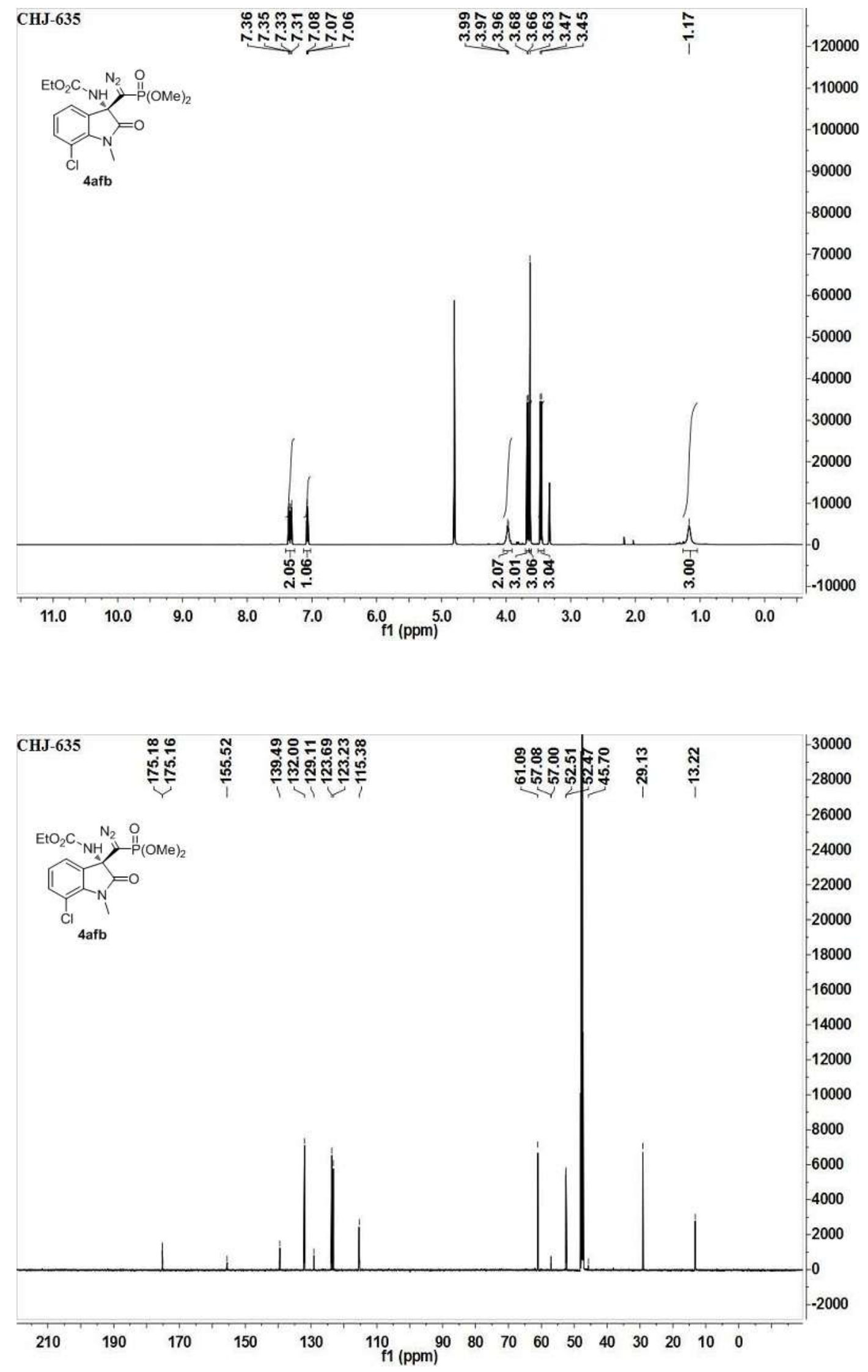

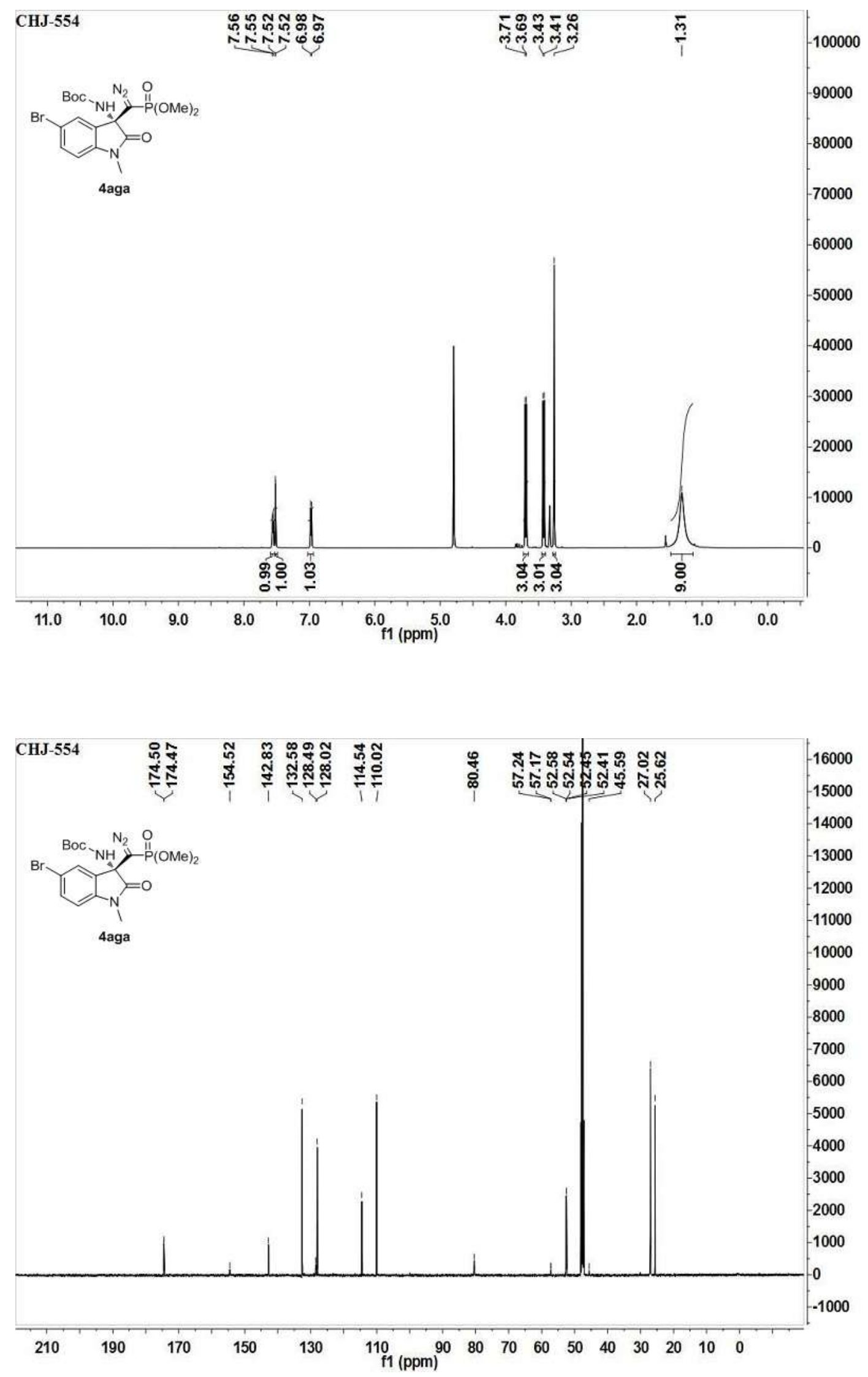

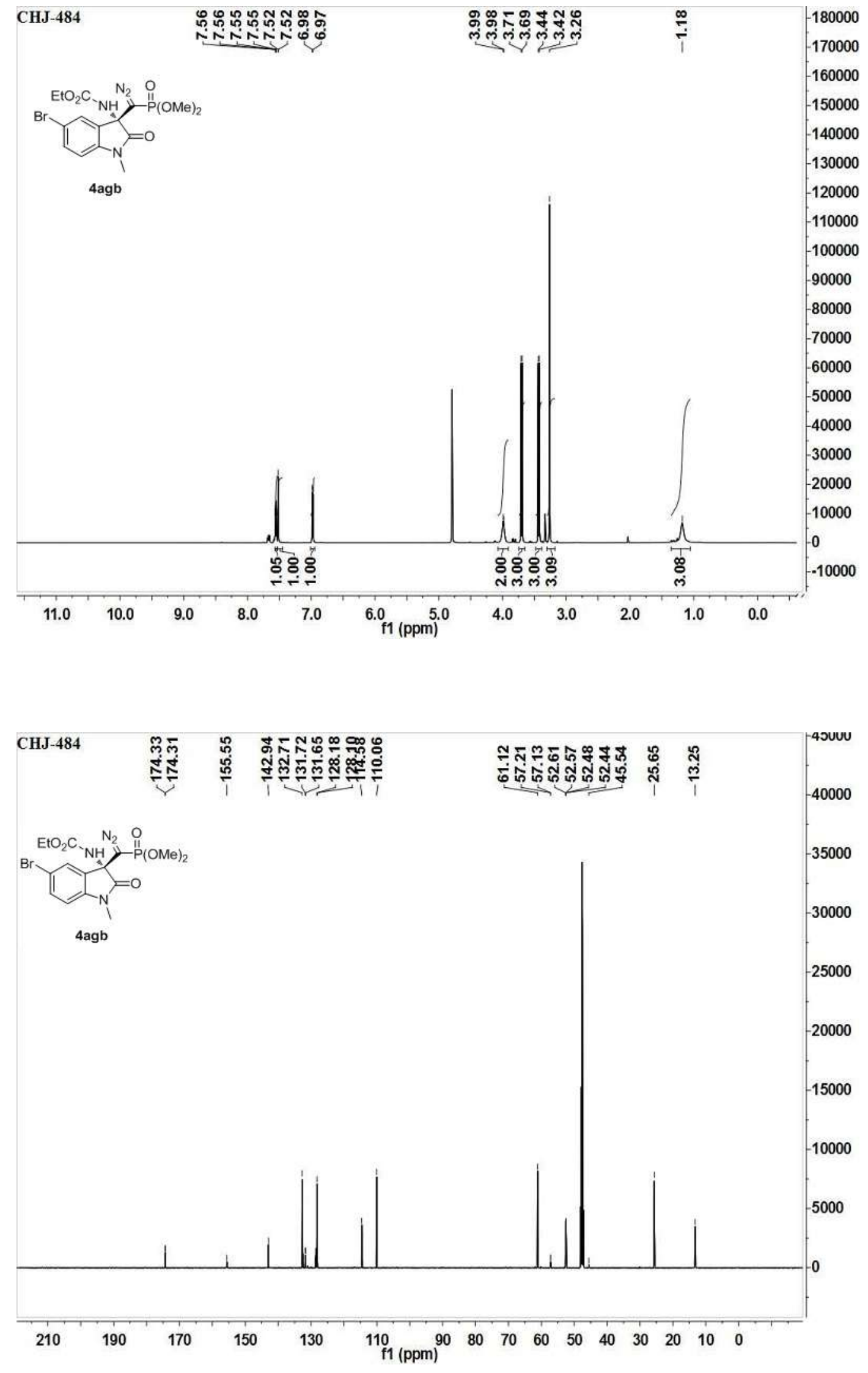

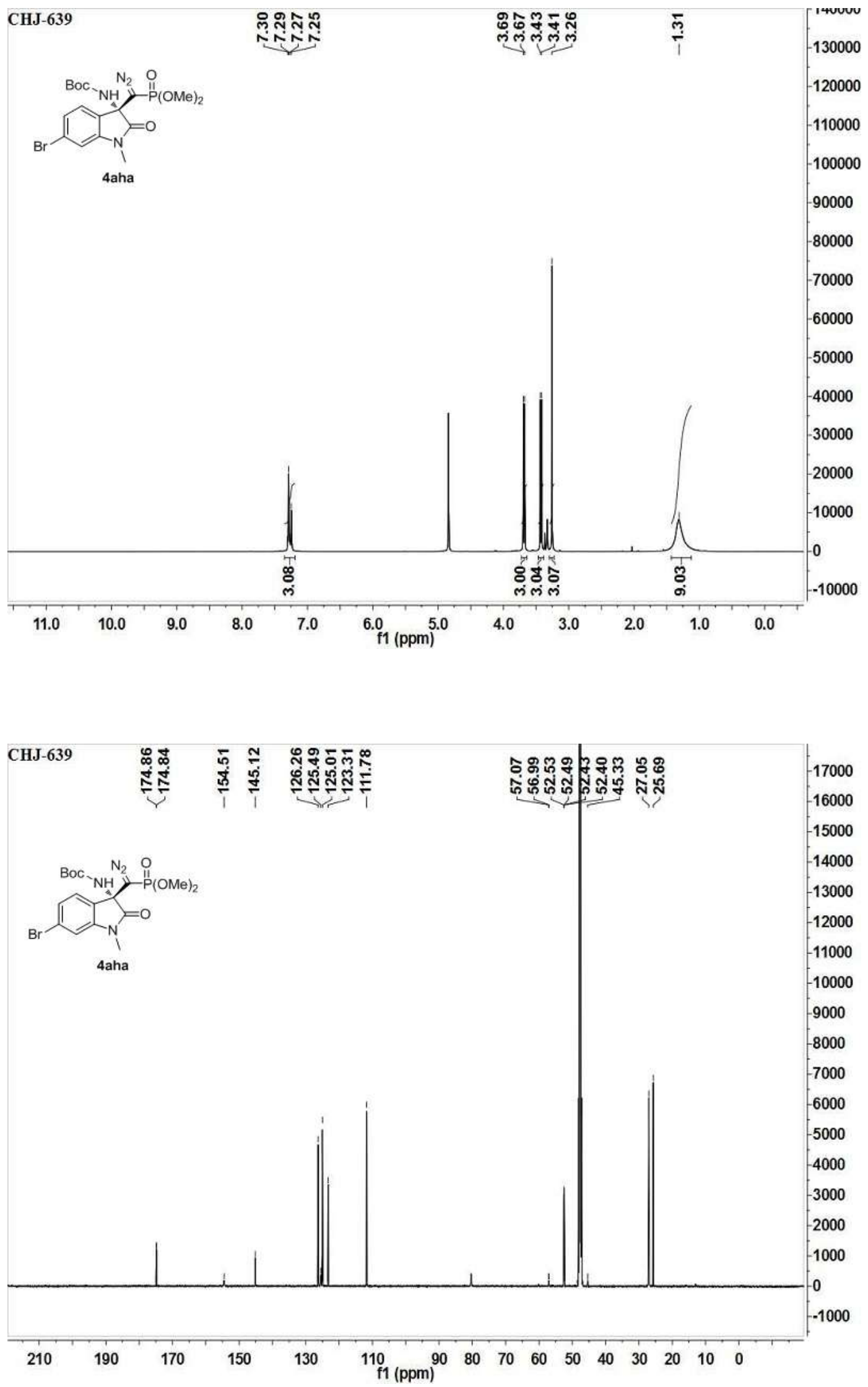

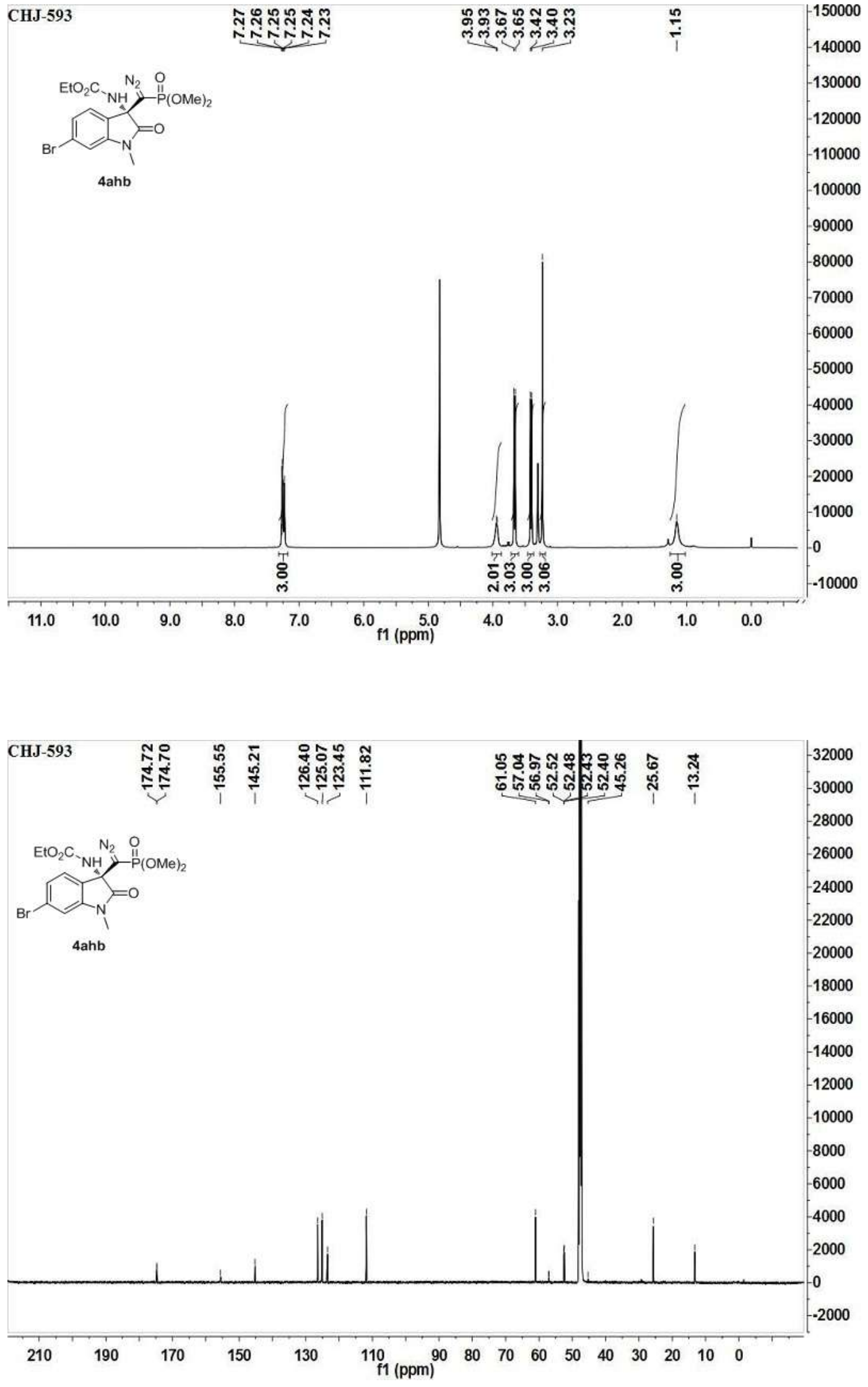

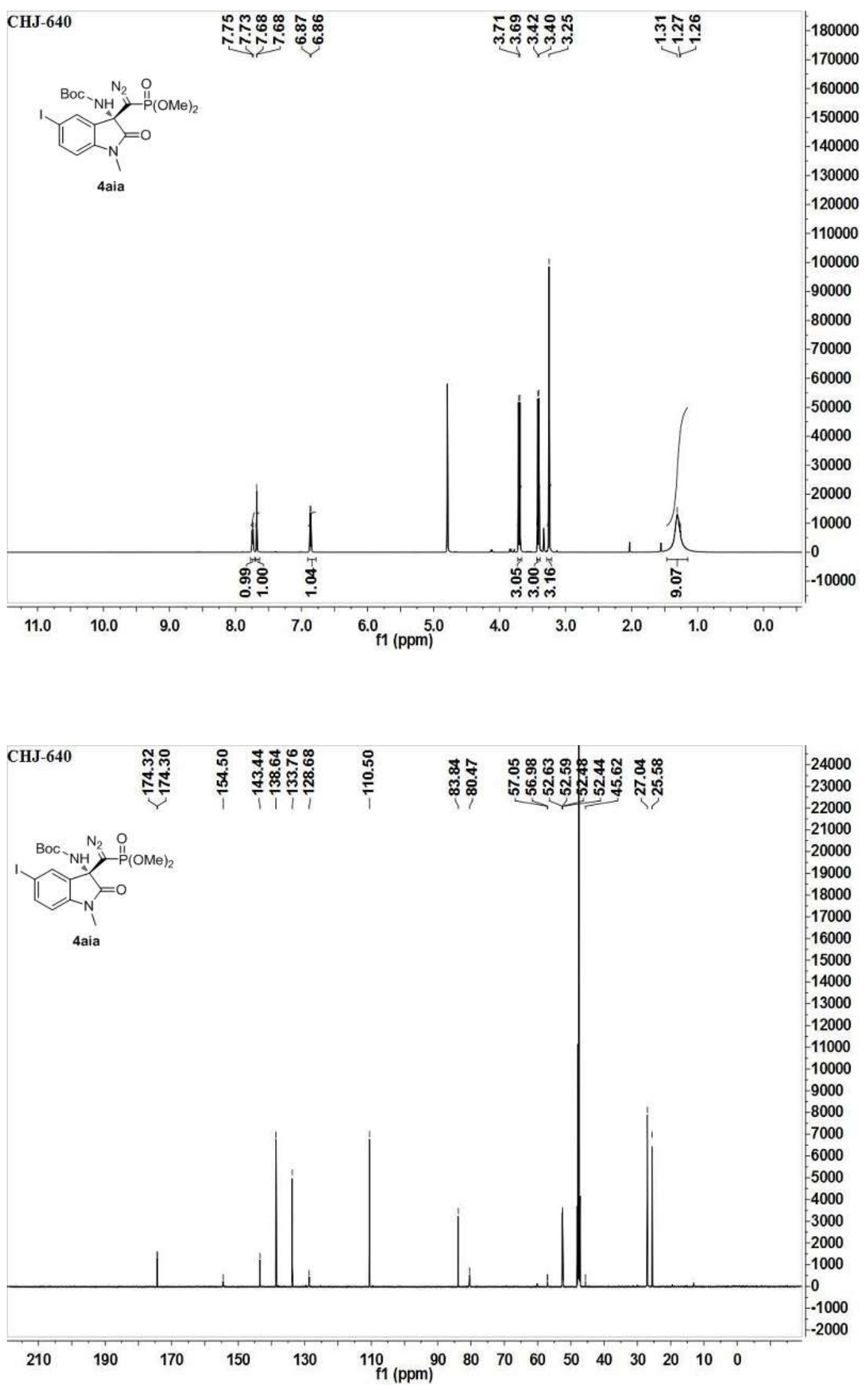

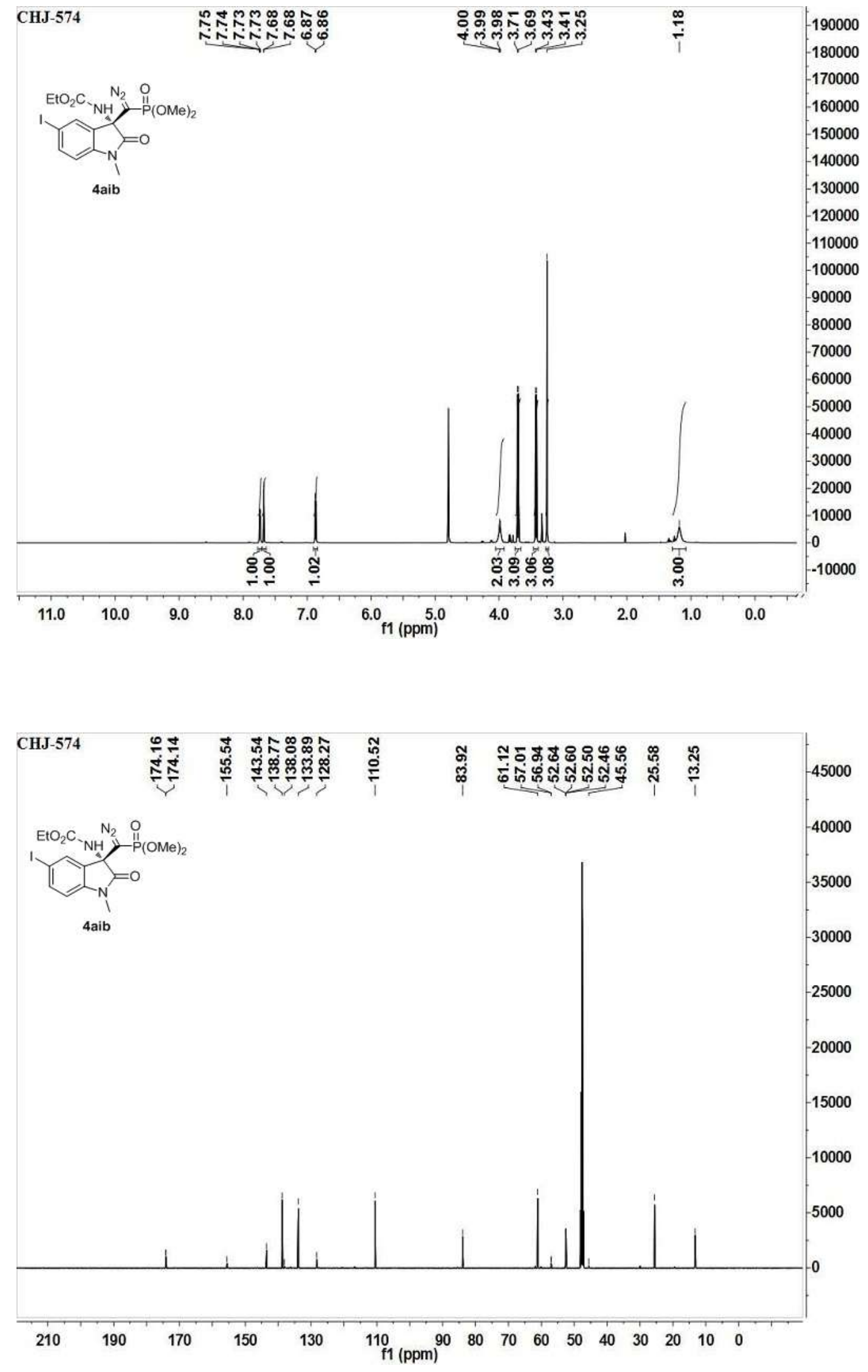

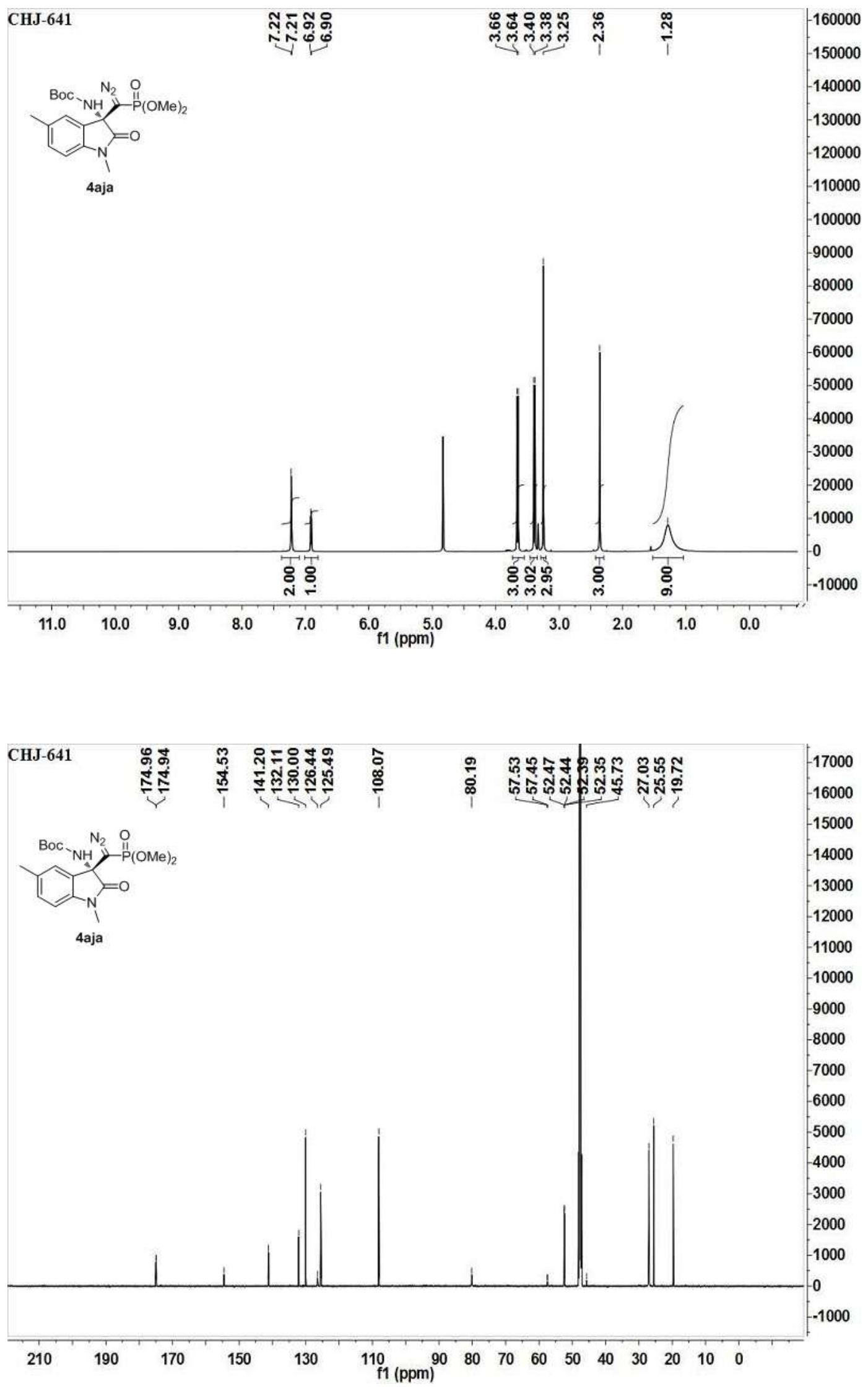

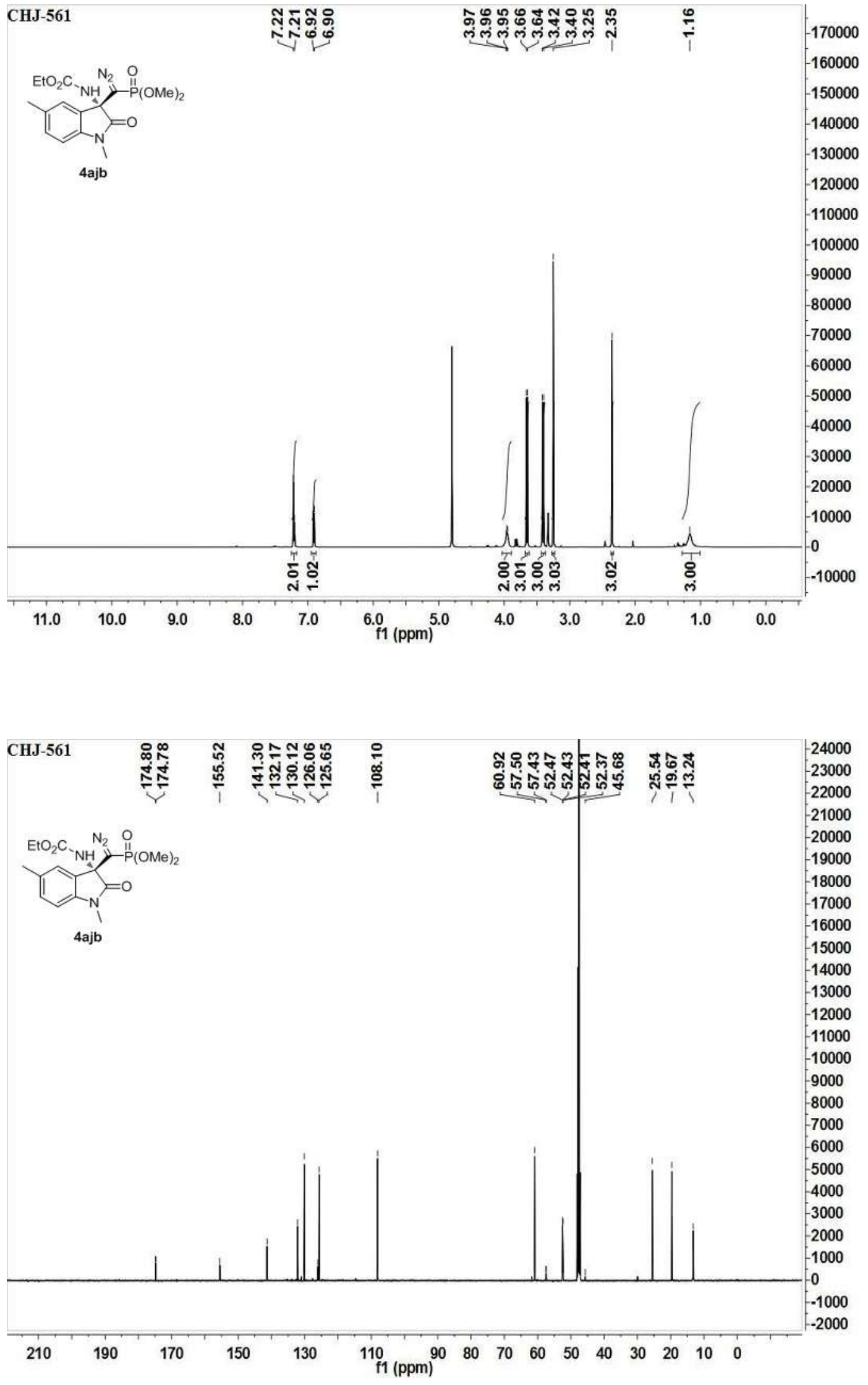

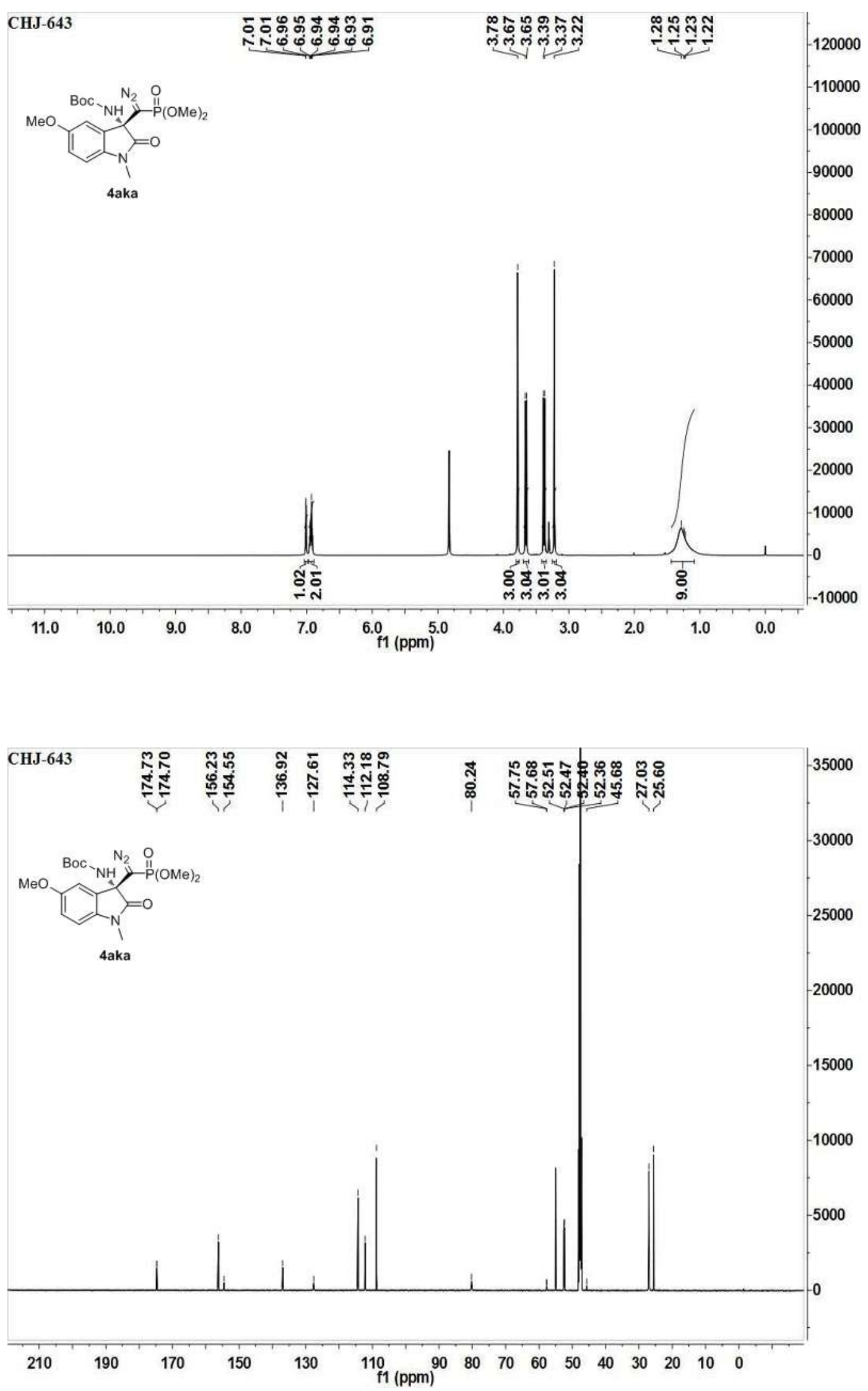

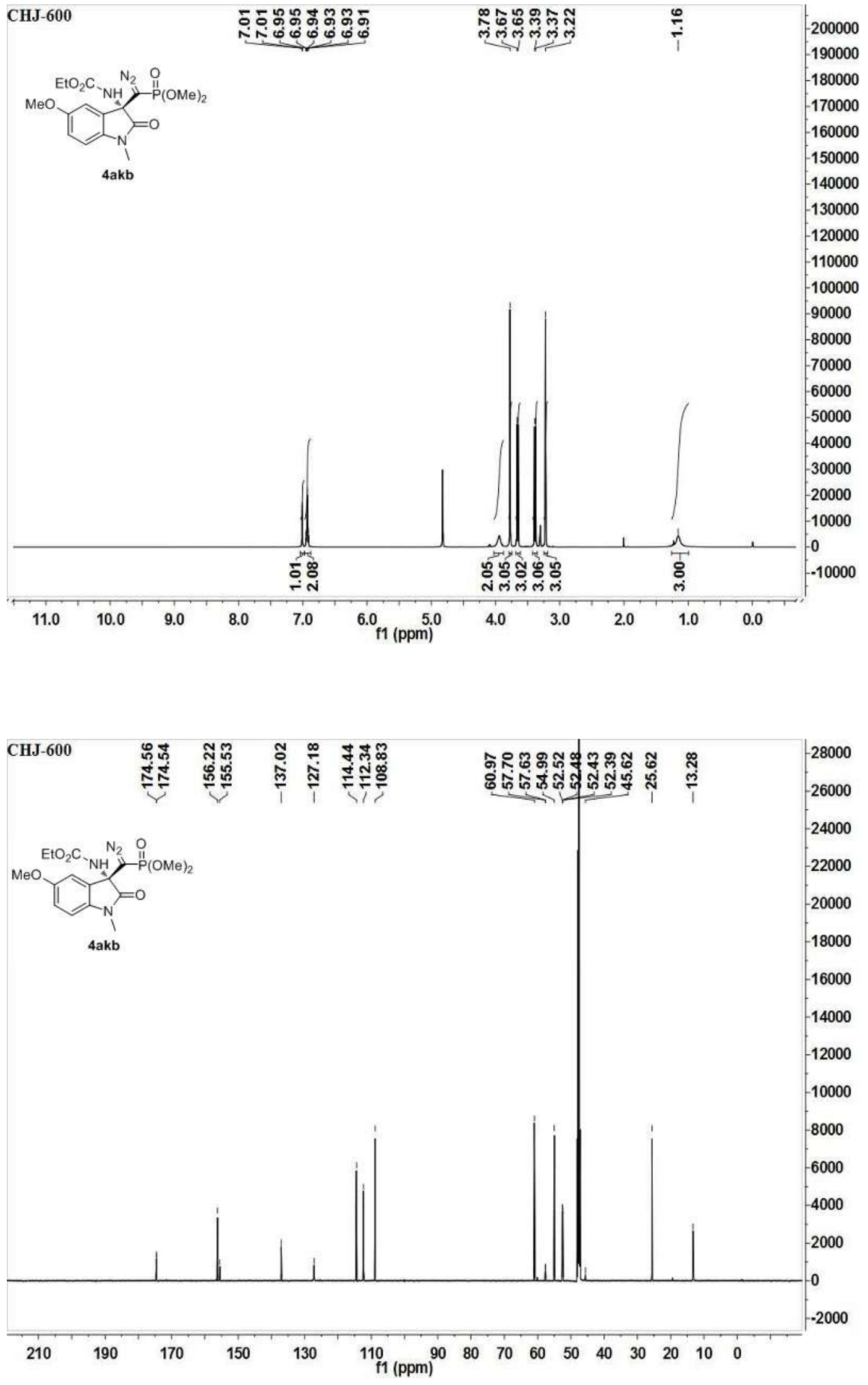

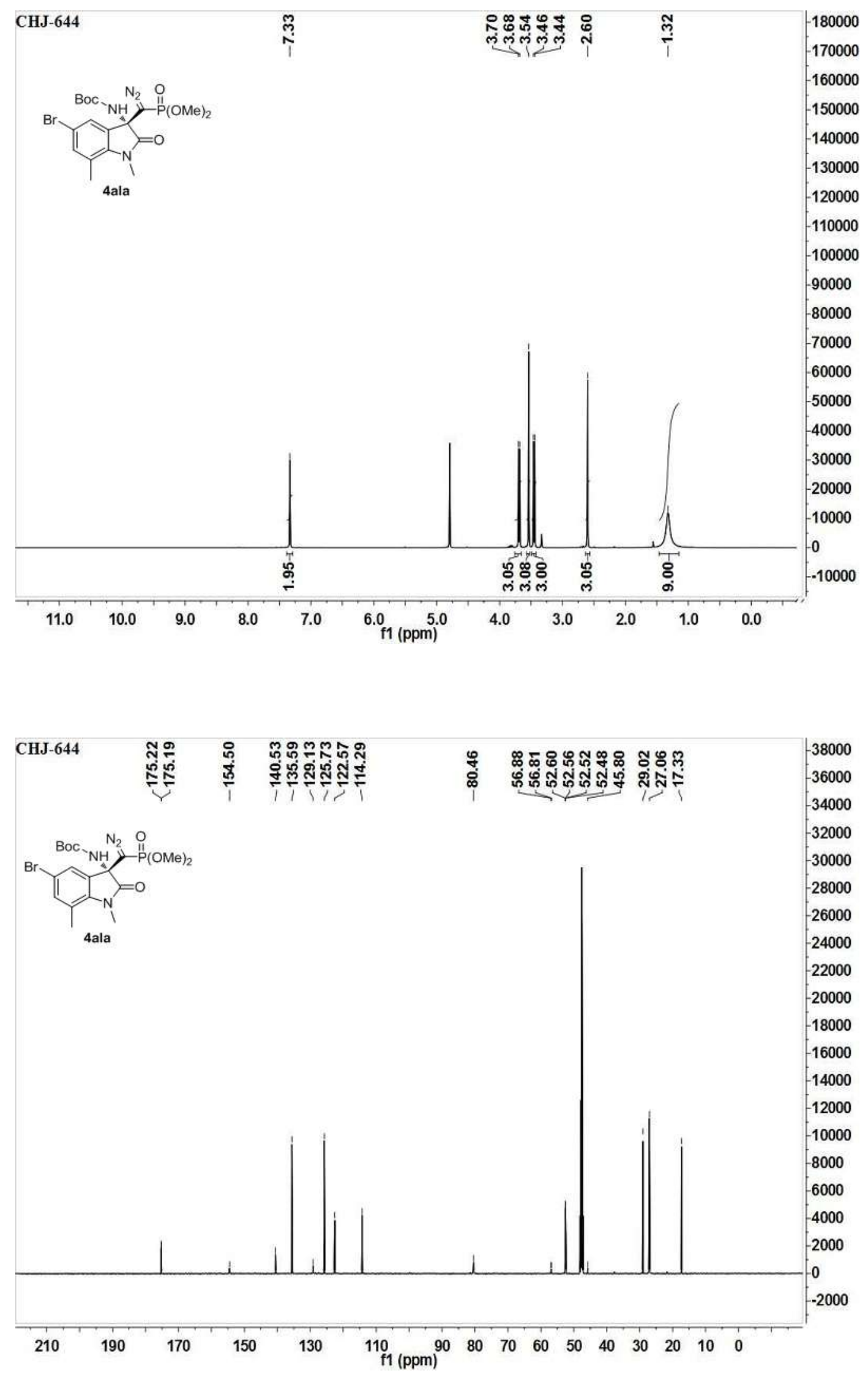

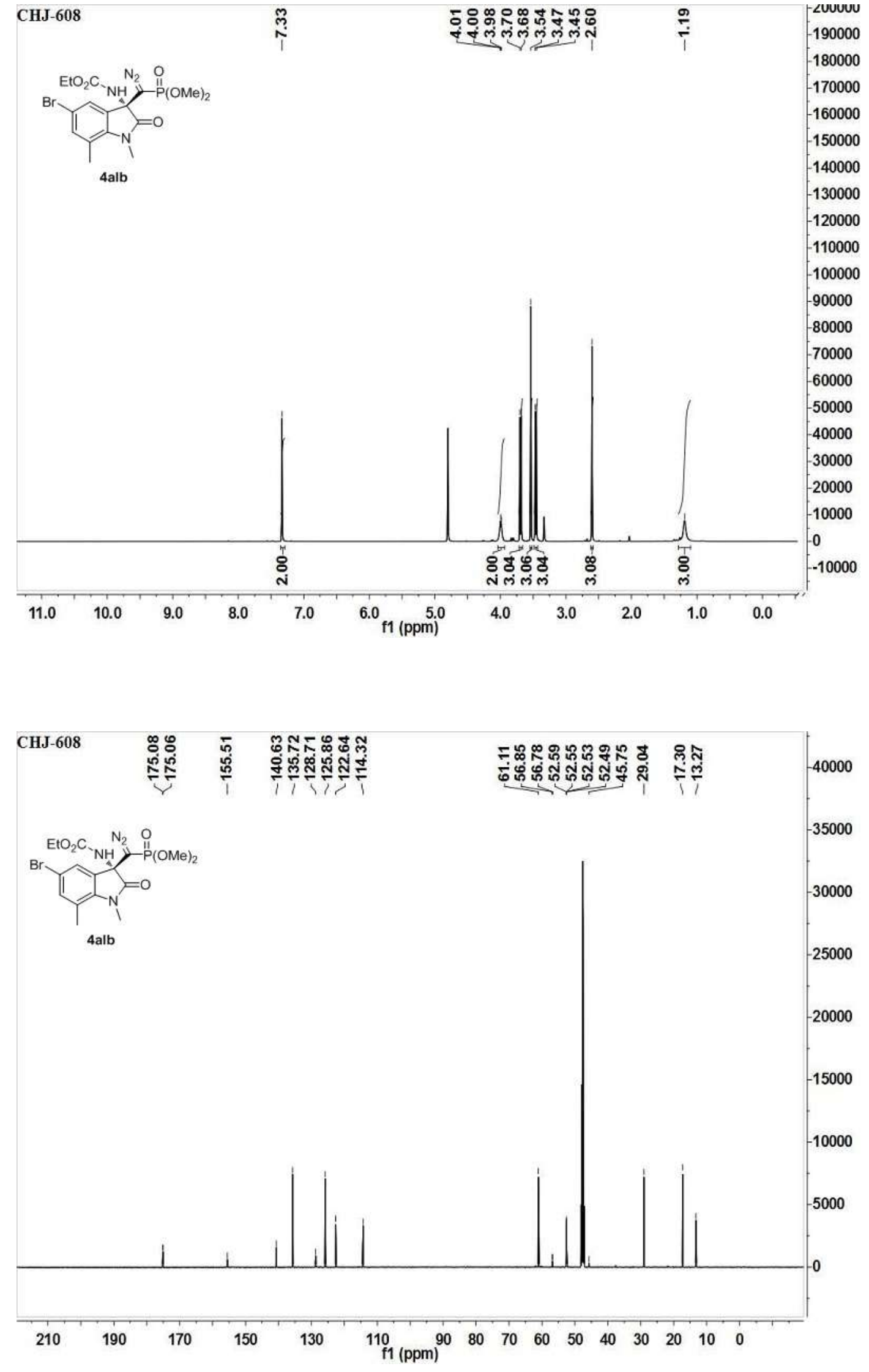

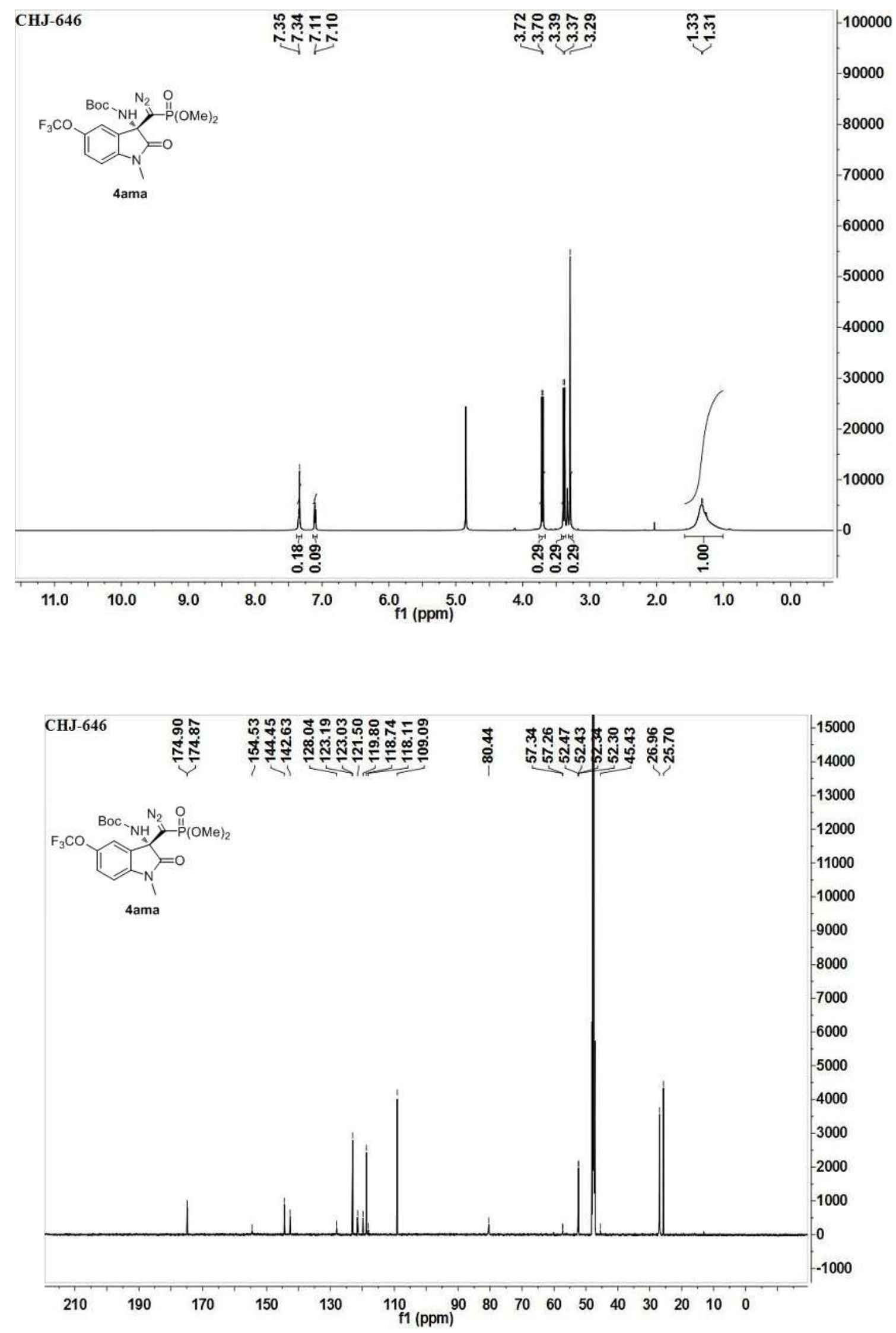

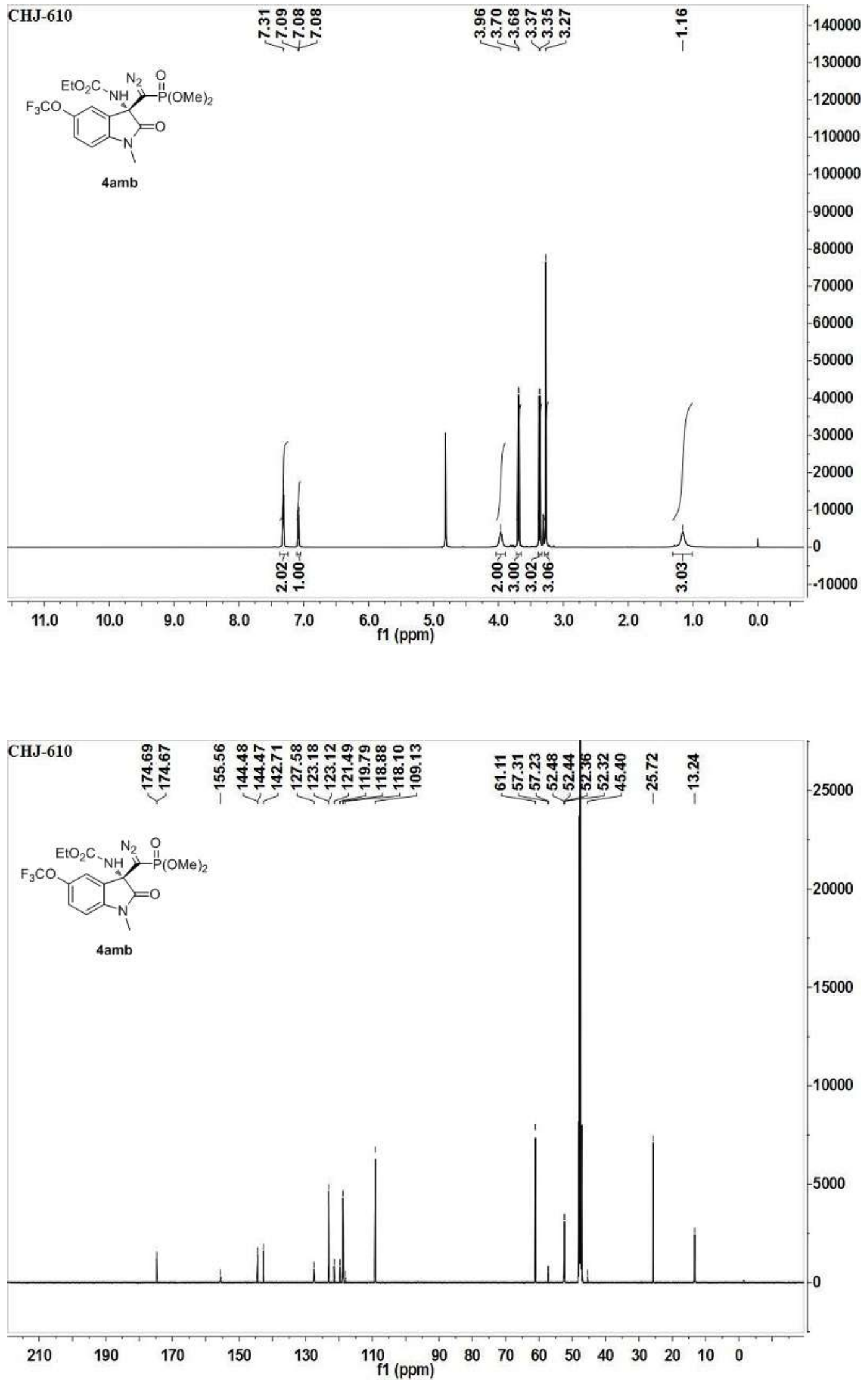

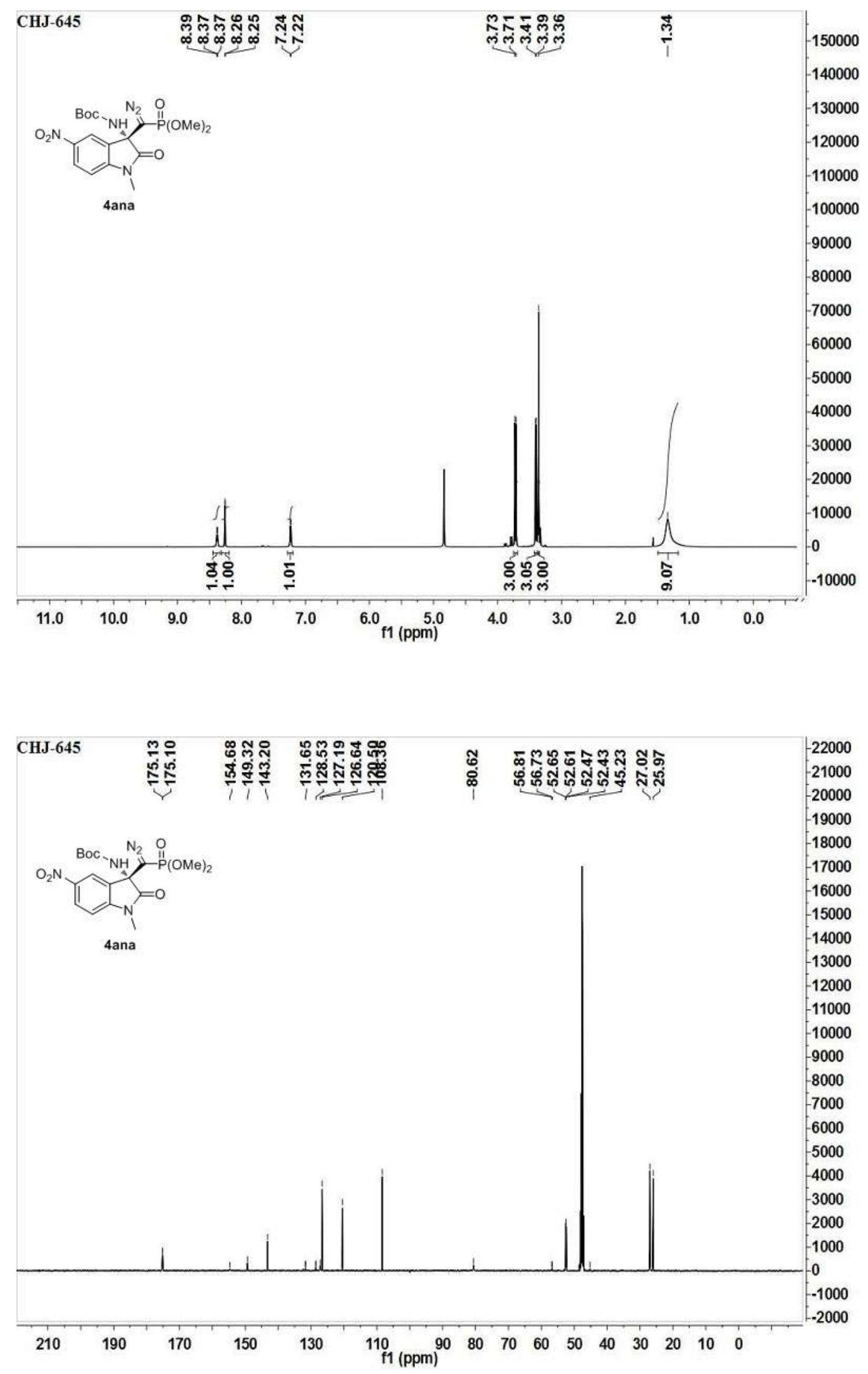

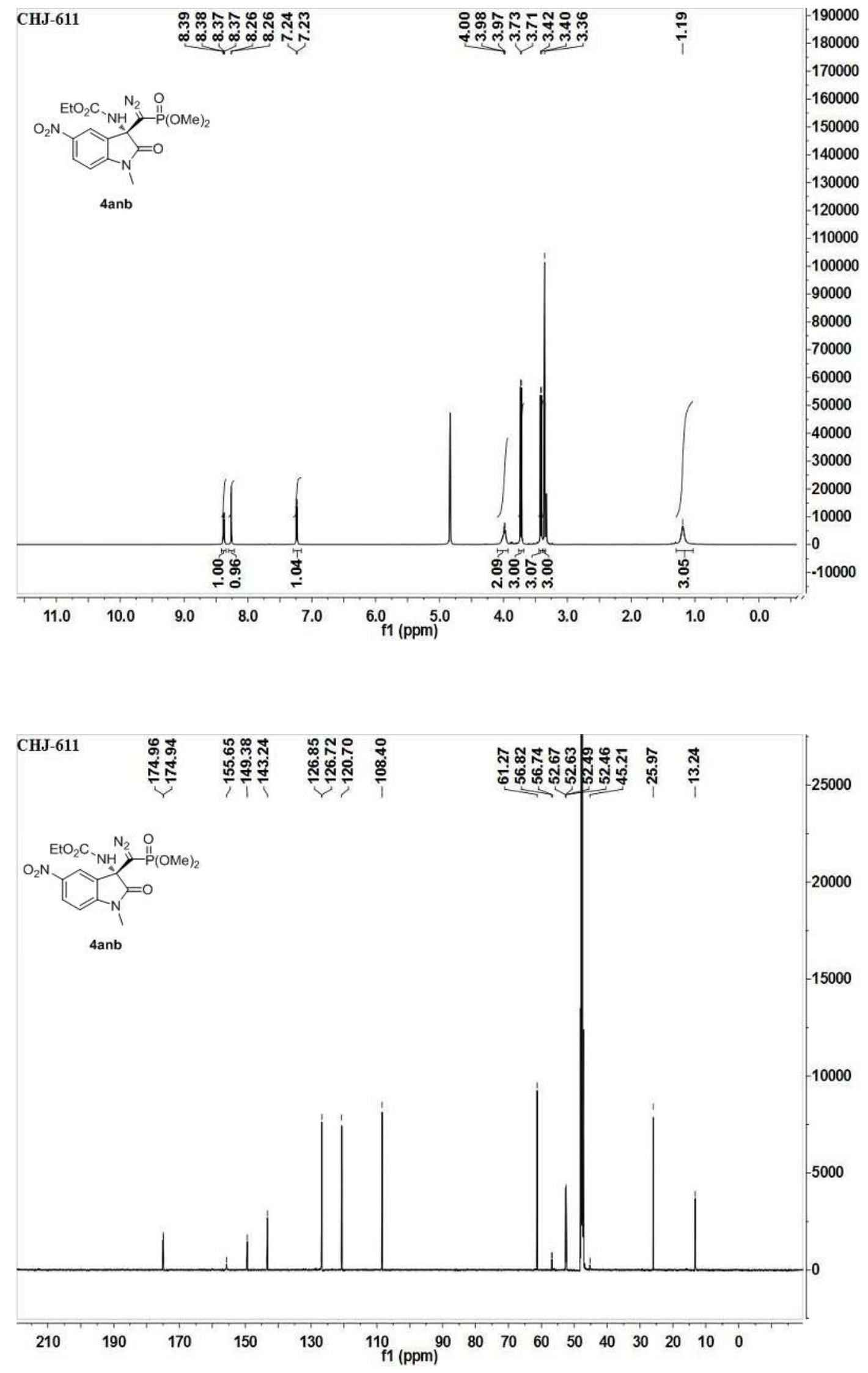

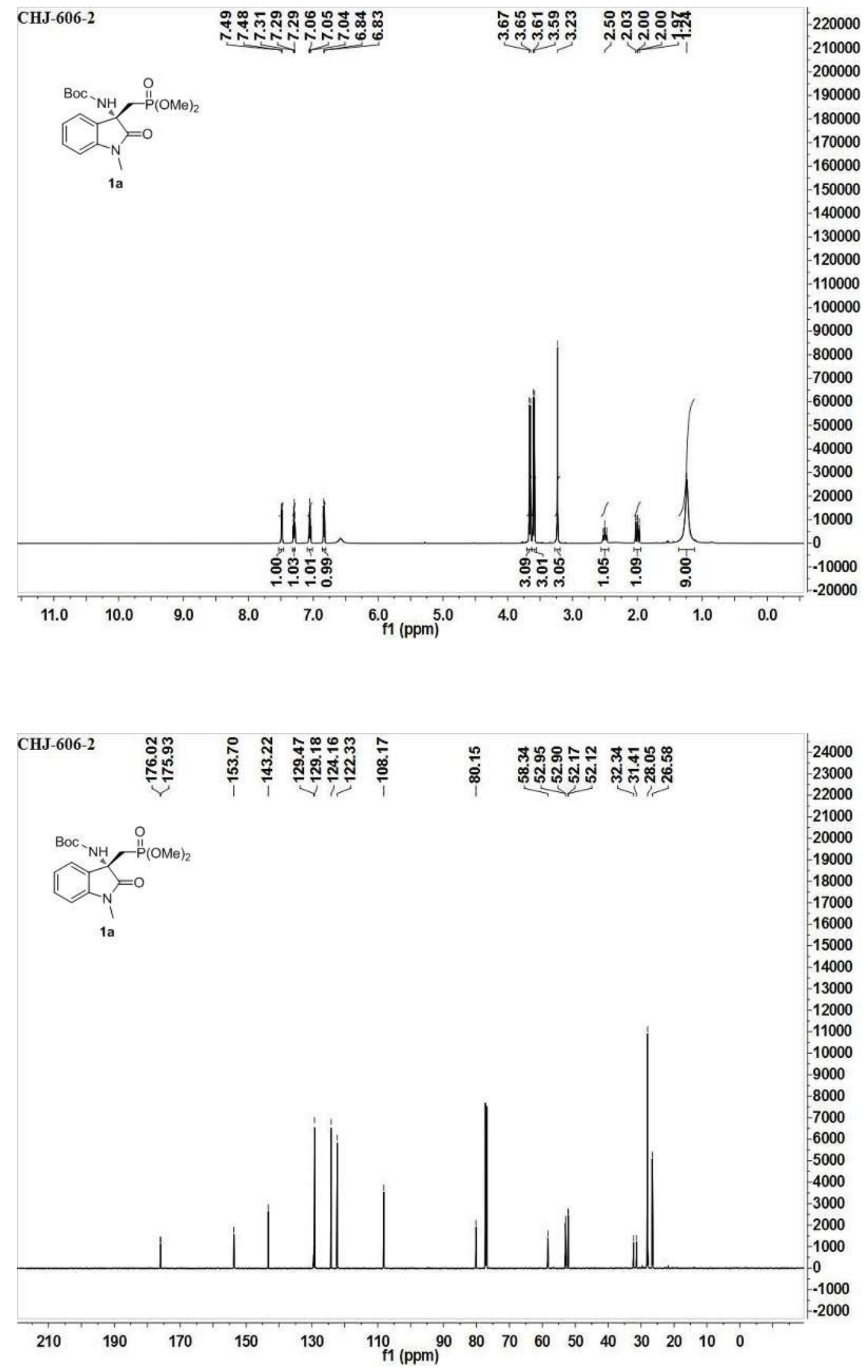

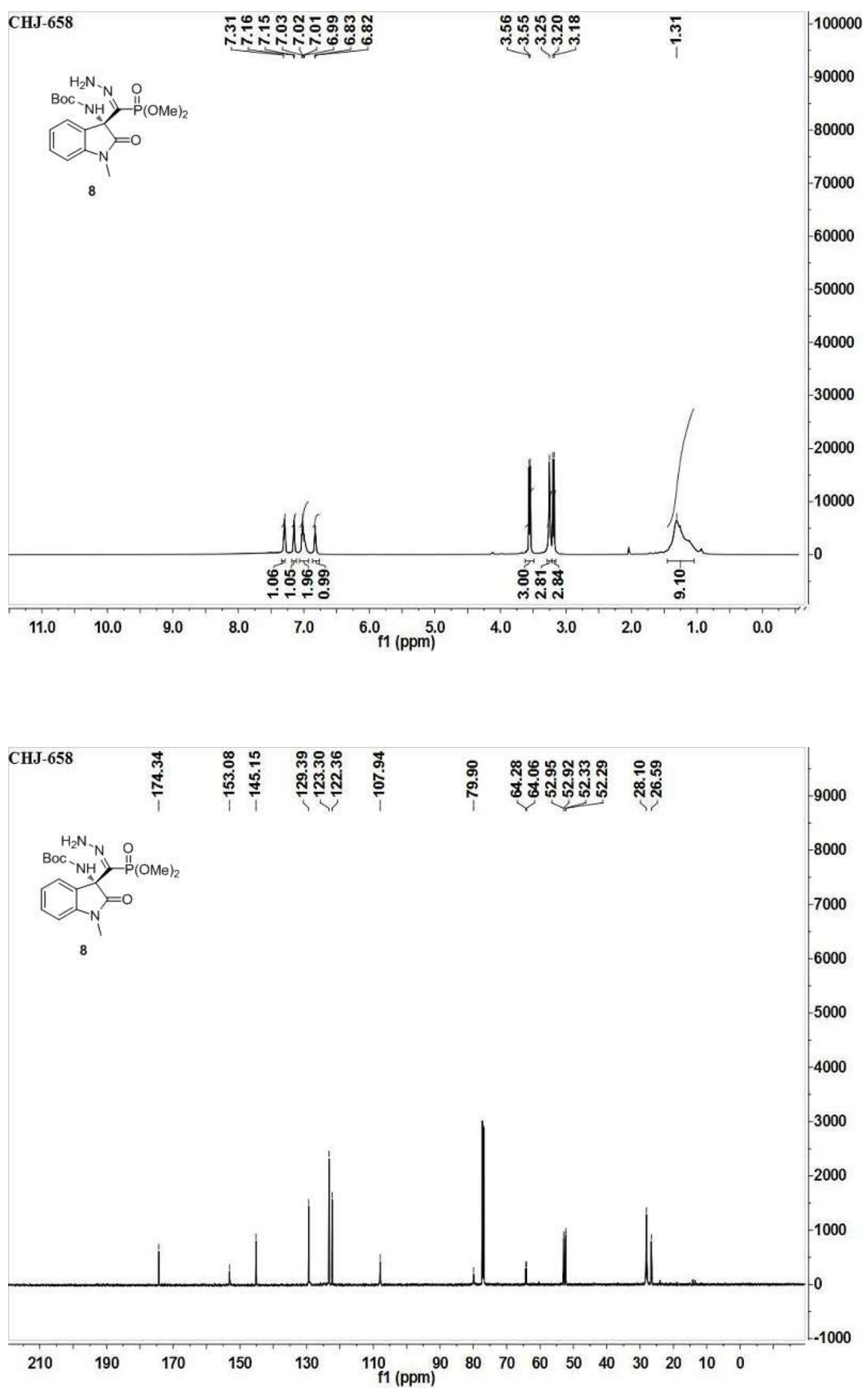

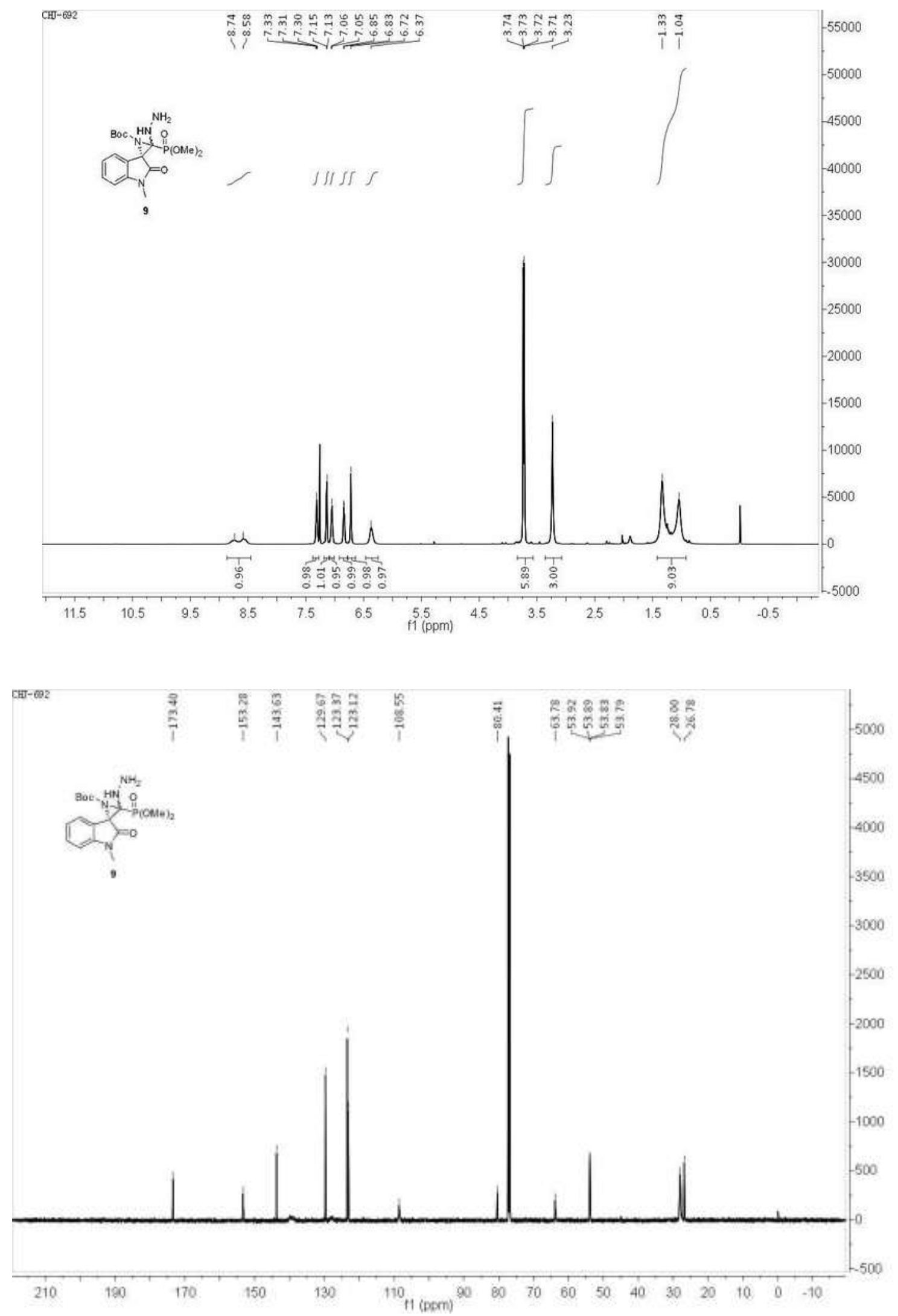


\section{HPLC Chromatogram of the Products}

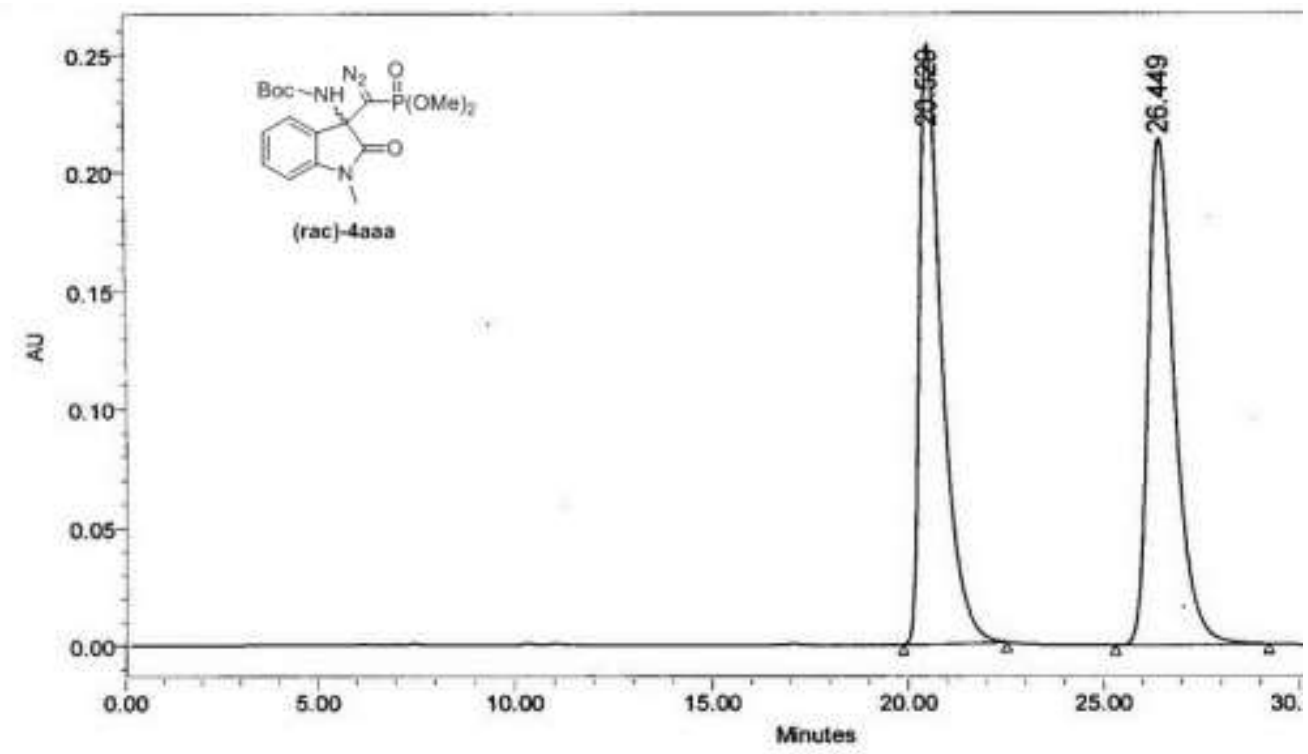

\begin{tabular}{|c|c|c|c|c|c|}
\hline & $\begin{array}{c}\text { RT } \\
(\mathrm{min})\end{array}$ & $\begin{array}{c}\text { Area } \\
\left(\mu \mathrm{V}^{*} \text { sec }\right)\end{array}$ & \% Area & $\begin{array}{c}\text { Height } \\
(\mu \mathrm{V})\end{array}$ & $\begin{array}{c}\% \\
\text { Height }\end{array}$ \\
\hline 1 & 20.529 & 10045536 & 50.13 & 253694 & 54.31 \\
\hline 2 & 26.449 & 9993985 & 49.87 & 213436 & 45.69 \\
\hline
\end{tabular}

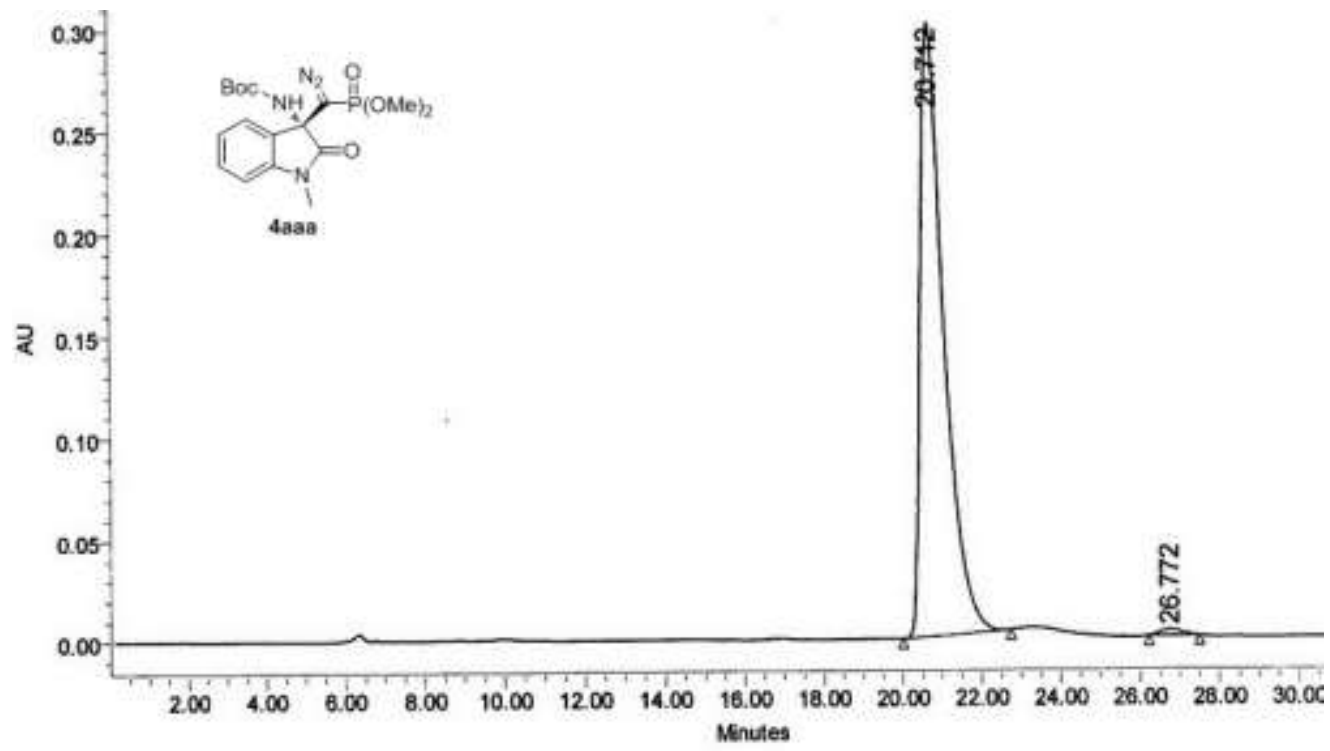

\begin{tabular}{|c|c|r|r|r|r|}
\hline & $\begin{array}{c}\text { Rा } \\
(\mathrm{min})\end{array}$ & $\begin{array}{c}\text { Area } \\
\left(\mu \mathrm{V}^{*} \mathrm{sec}\right)\end{array}$ & $\%$ Area & $\begin{array}{c}\text { Height } \\
(\mu \mathrm{N})\end{array}$ & $\begin{array}{c}\% \\
\text { Height }\end{array}$ \\
\hline 1 & 20.712 & 12473938 & 99.06 & 300856 & 99.02 \\
\hline 2 & 26.772 & 118786 & 0.94 & 2970 & 0.98 \\
\hline
\end{tabular}




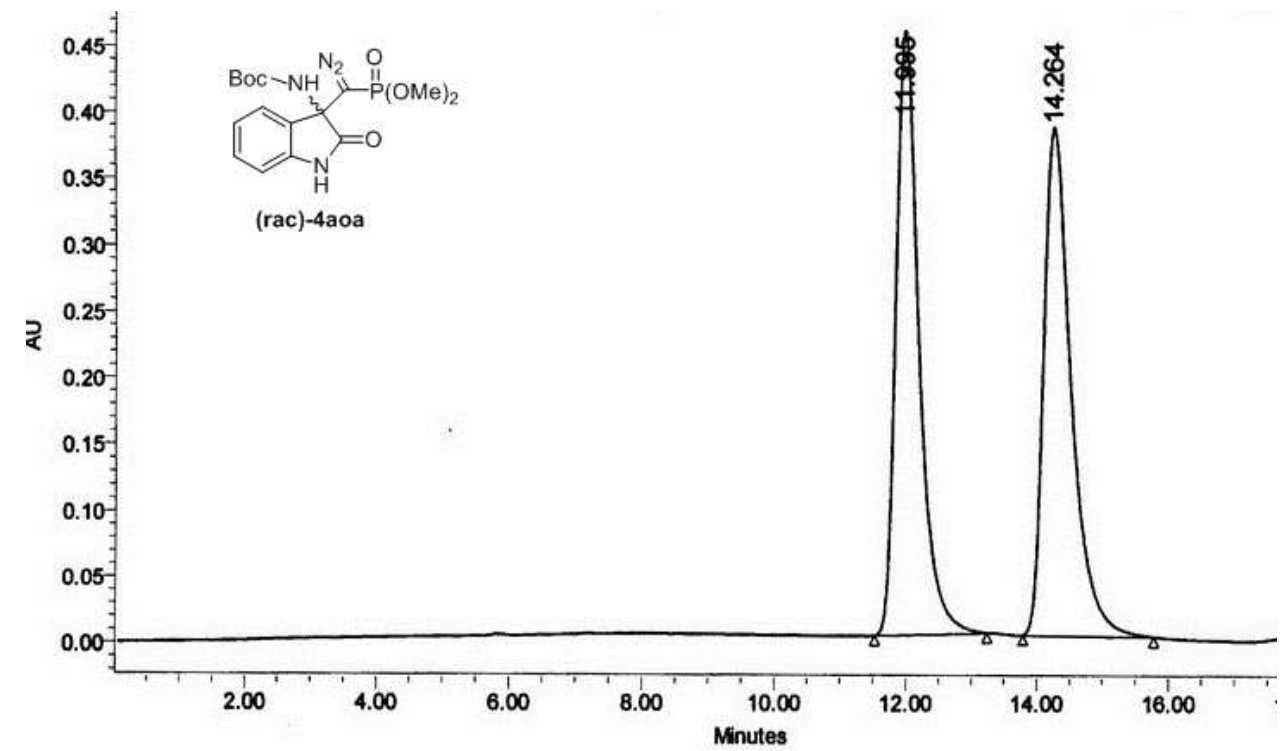

\begin{tabular}{|c|c|c|c|c|c|}
\hline & $\begin{array}{c}\mathrm{RT} \\
(\mathrm{min})\end{array}$ & $\begin{array}{c}\text { Area } \\
(\mu \mathrm{V} * \mathrm{sec})\end{array}$ & $\%$ Area & $\begin{array}{c}\text { Height } \\
(\mu \mathrm{V})\end{array}$ & $\begin{array}{c}\% \\
\text { Height }\end{array}$ \\
\hline 1 & 11.995 & 11127150 & 50.04 & 456110 & 54.34 \\
\hline 2 & 14.264 & 11109327 & 49.96 & 383250 & 45.66 \\
\hline
\end{tabular}

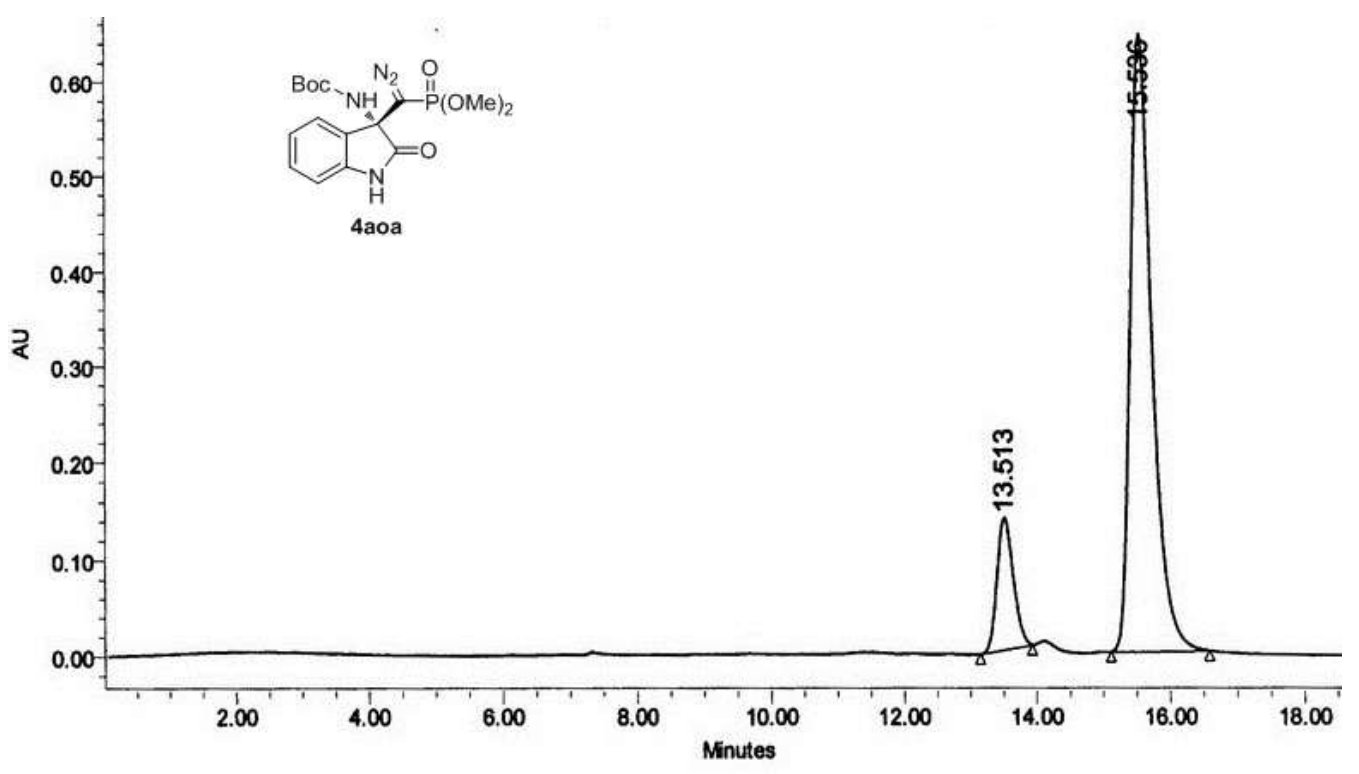

\begin{tabular}{|c|c|c|c|c|c|}
\hline & $\begin{array}{c}\mathrm{RT} \\
(\mathrm{min})\end{array}$ & $\begin{array}{c}\text { Area } \\
\left(\mu \mathrm{V}^{*} \mathrm{sec}\right)\end{array}$ & $\%$ Area & $\begin{array}{c}\text { Height } \\
(\mu \mathrm{V})\end{array}$ & $\begin{array}{c}\% \\
\text { Height }\end{array}$ \\
\hline 1 & 13.513 & 2343544 & 14.06 & 137305 & 17.52 \\
\hline 2 & 15.536 & 14330223 & 85.94 & 646582 & 82.48 \\
\hline
\end{tabular}




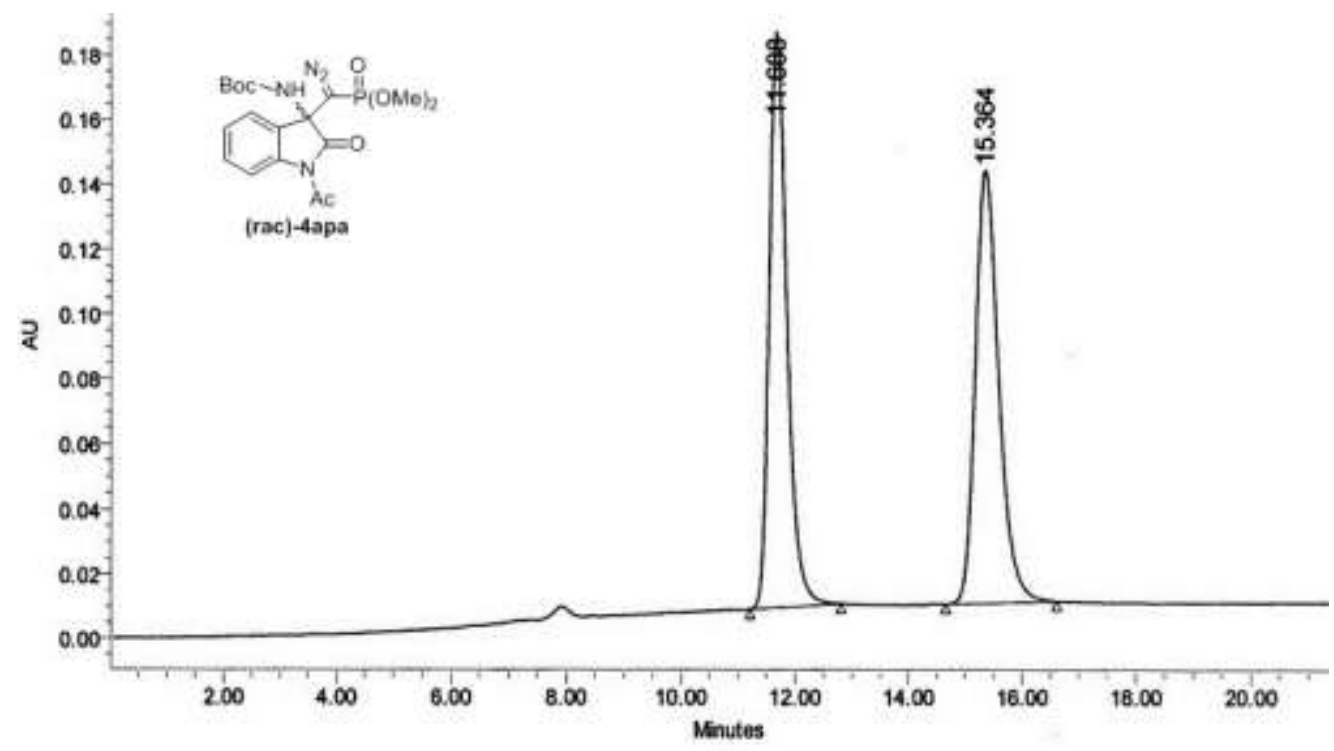

\begin{tabular}{|c|c|c|c|c|c|}
\hline & $\begin{array}{c}\text { RT } \\
(\mathrm{min})\end{array}$ & $\begin{array}{c}\text { Area } \\
(\mu \mathrm{V} \text { "sec })\end{array}$ & $\%$ Area & $\begin{array}{c}\text { Height } \\
(\mu \mathrm{V})\end{array}$ & $\begin{array}{c}\% \\
\text { Height }\end{array}$ \\
\hline 1 & 11.688 & 3840446 & 50.11 & 177762 & 57.15 \\
\hline 2 & 15.364 & 3823751 & 49.89 & 133273 & 42.85 \\
\hline
\end{tabular}

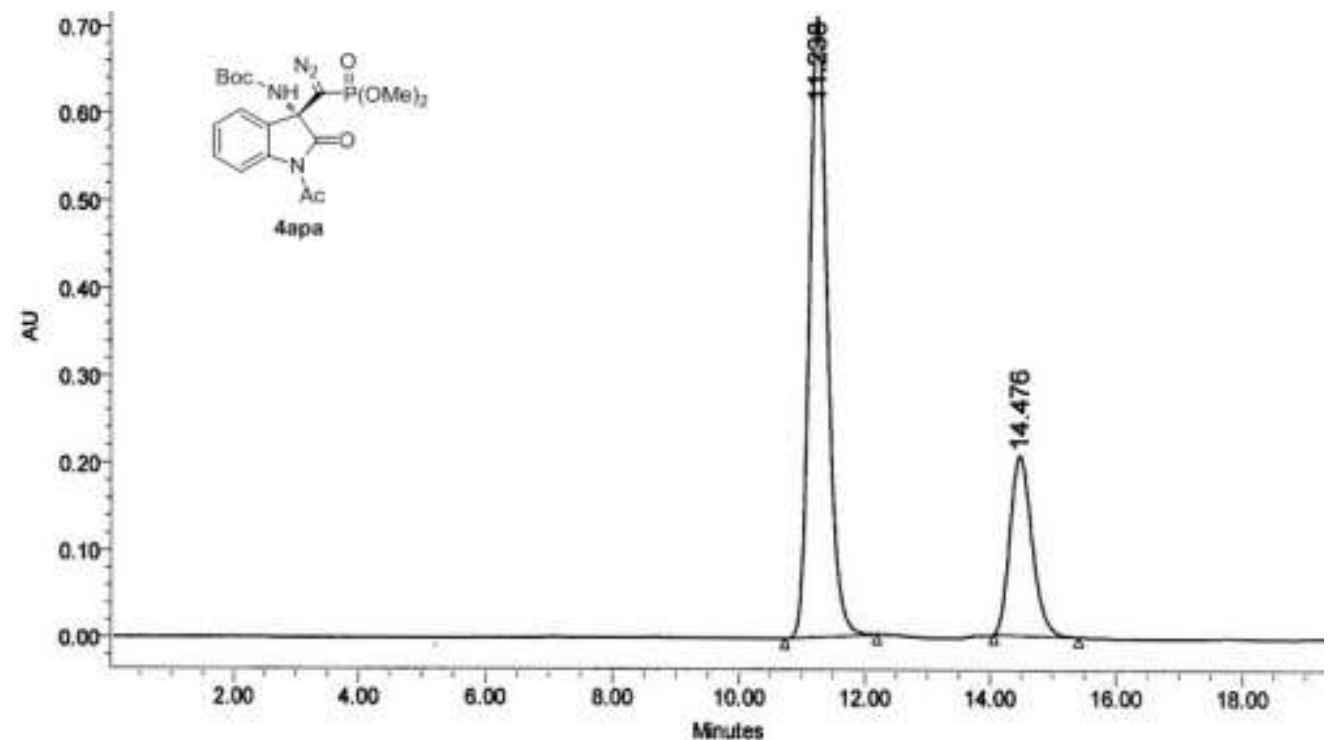

\begin{tabular}{|c|c|c|c|c|c|}
\hline & $\begin{array}{c}\text { RT } \\
(\mathrm{min})\end{array}$ & $\begin{array}{c}\text { Area } \\
(\mu \mathrm{N} \text { 'sec) }\end{array}$ & $\%$ Area & $\begin{array}{c}\text { Height } \\
(\mu \mathrm{N})\end{array}$ & $\begin{array}{c}\% \\
\text { Height }\end{array}$ \\
\hline 1 & 11.238 & 14165380 & 73.69 & 710955 & 77.54 \\
\hline 2 & 14.476 & 5057087 & 26.31 & 205972 & 22.46 \\
\hline
\end{tabular}




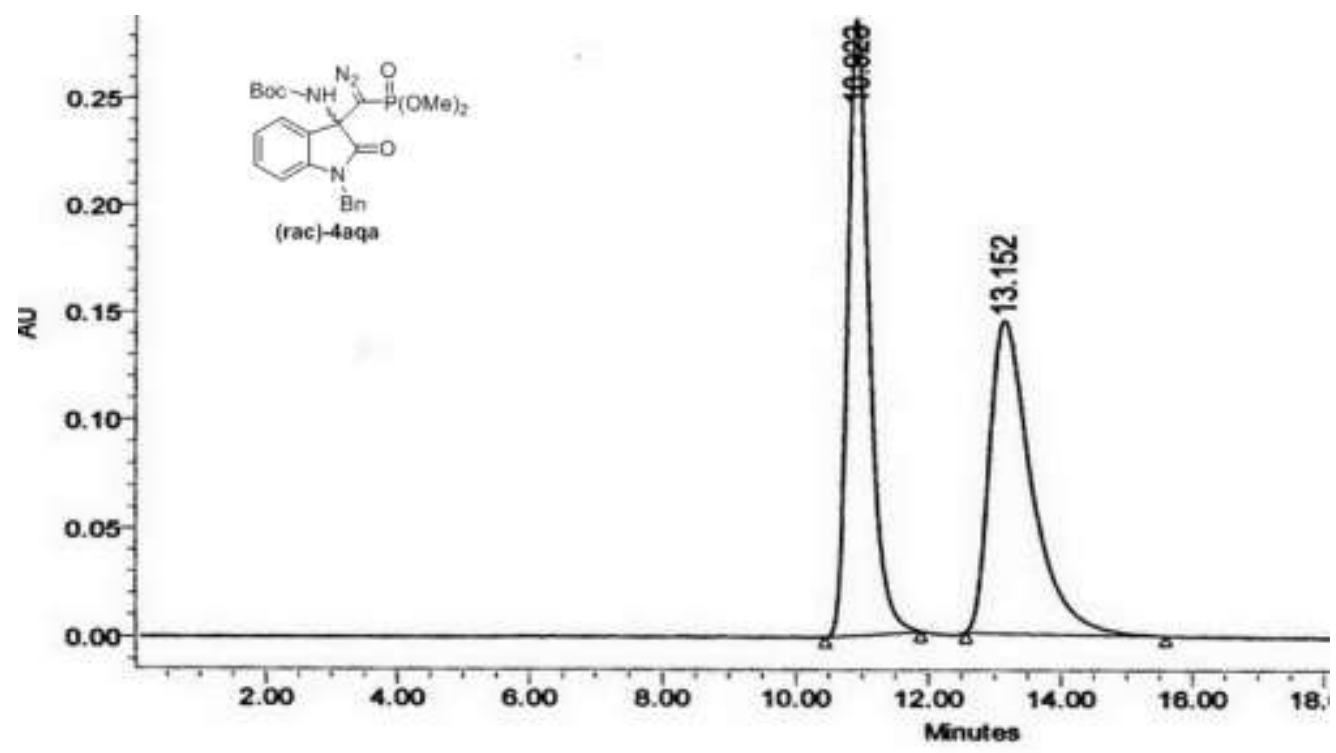

\begin{tabular}{|c|c|c|c|c|c|}
\hline & $\begin{array}{c}\text { RT } \\
(\min )\end{array}$ & $\begin{array}{c}\text { Area } \\
\left(\mu \mathrm{V}^{*} \text { sec }\right)\end{array}$ & \% Area & $\begin{array}{c}\text { Height } \\
(\mu \mathrm{V})\end{array}$ & $\begin{array}{c}\% \\
\text { Height }\end{array}$ \\
\hline 1 & 10.923 & 6437136 & 50.18 & 287812 & 66.50 \\
\hline 2 & 13.152 & 6390447 & 49.82 & 145008 & 33.50 \\
\hline
\end{tabular}

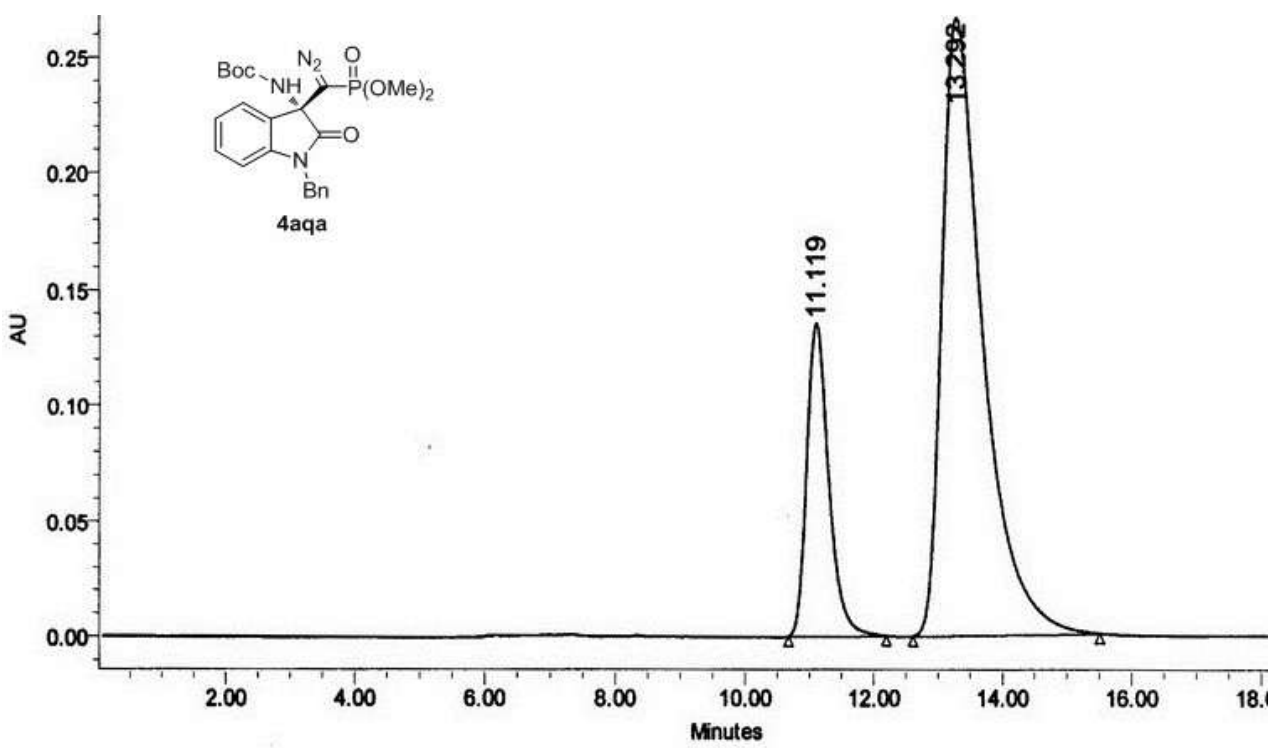

\begin{tabular}{|c|c|c|c|c|c|}
\hline & $\begin{array}{c}\text { RT } \\
(\mathrm{min})\end{array}$ & $\begin{array}{c}\text { Area } \\
\left(\mu \mathrm{V}^{*} \mathrm{sec}\right)\end{array}$ & $\%$ Area & $\begin{array}{c}\text { Height } \\
(\mu \mathrm{V})\end{array}$ & $\begin{array}{c}\% \\
\text { Height }\end{array}$ \\
\hline 1 & 11.119 & 3203850 & 21.36 & 135682 & 33.75 \\
\hline 2 & 13.292 & 11793577 & 78.64 & 266347 & 66.25 \\
\hline
\end{tabular}




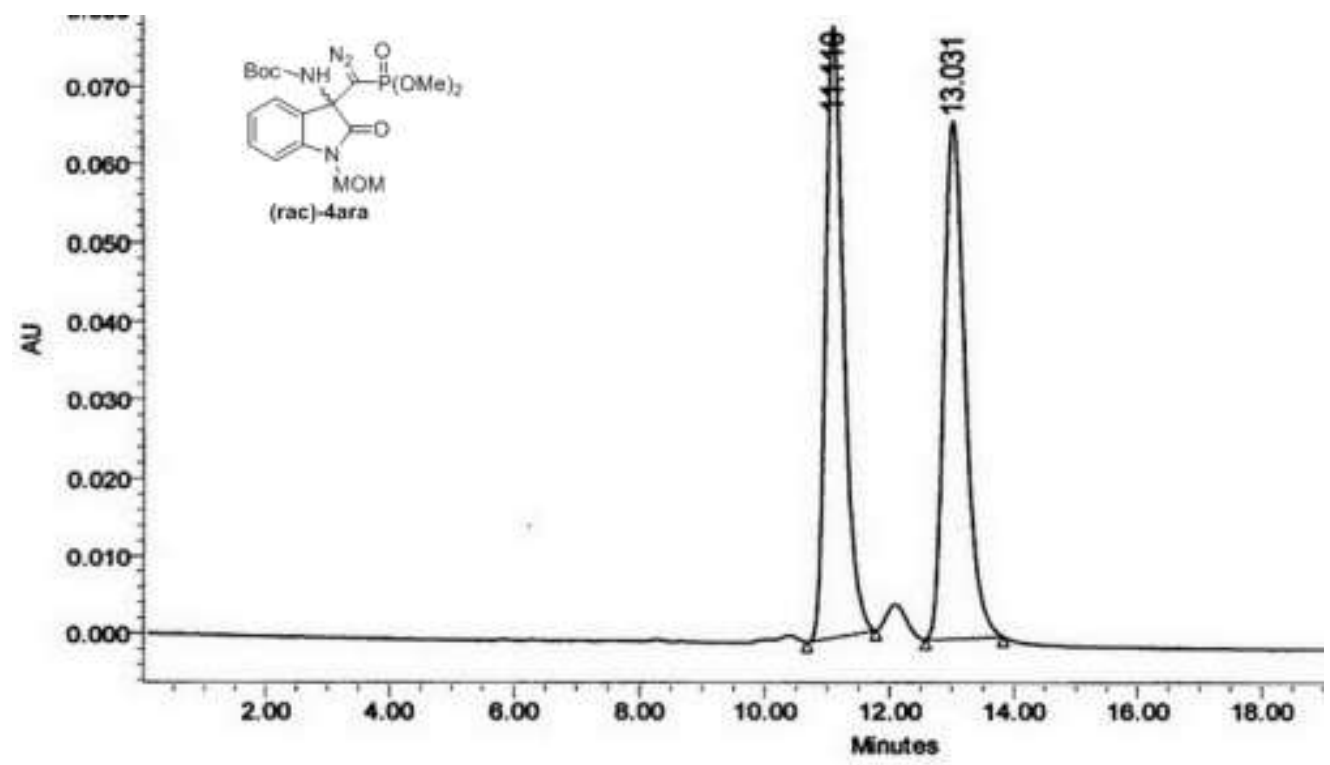

\begin{tabular}{|c|c|c|c|c|c|}
\hline & $\begin{array}{c}\text { Rा } \\
(\mathrm{min})\end{array}$ & $\begin{array}{c}\text { Area } \\
\left(\mu N^{*} \mathrm{sec}\right)\end{array}$ & $\%$ Area & $\begin{array}{c}\text { Height } \\
(\mu \mathrm{N})\end{array}$ & $\begin{array}{c}\% \\
\text { Height }\end{array}$ \\
\hline 1 & 11.110 & 1568133 & 50.02 & 78369 & 54.22 \\
\hline 2 & 13.031 & 1566566 & 49.98 & 66166 & 45.78 \\
\hline
\end{tabular}

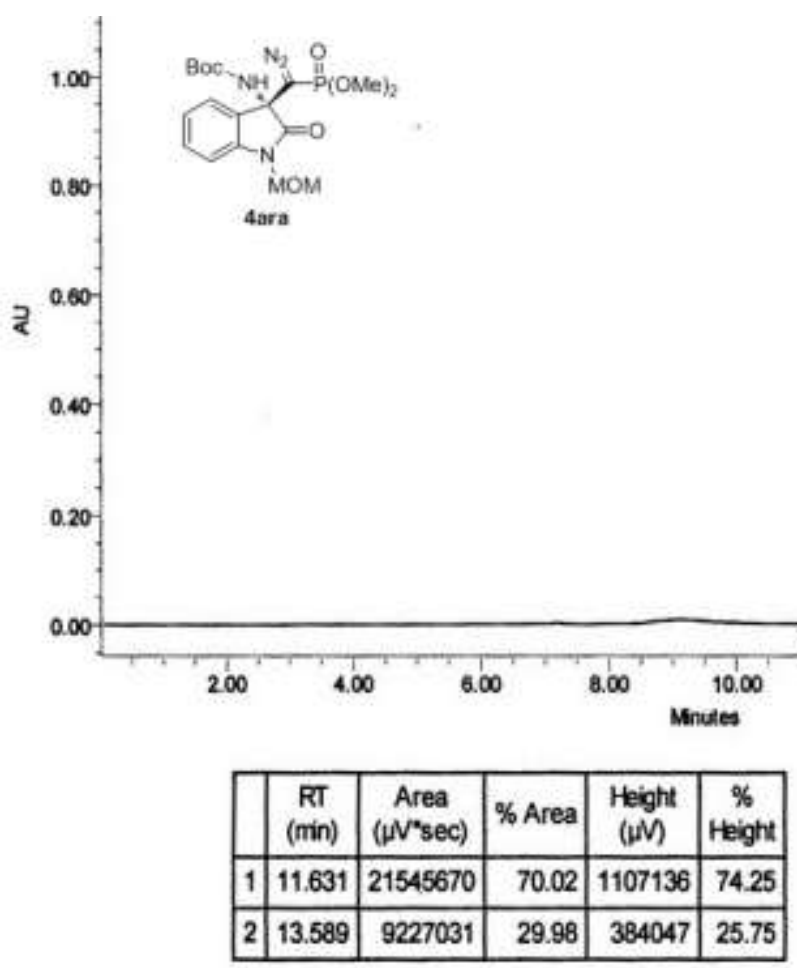




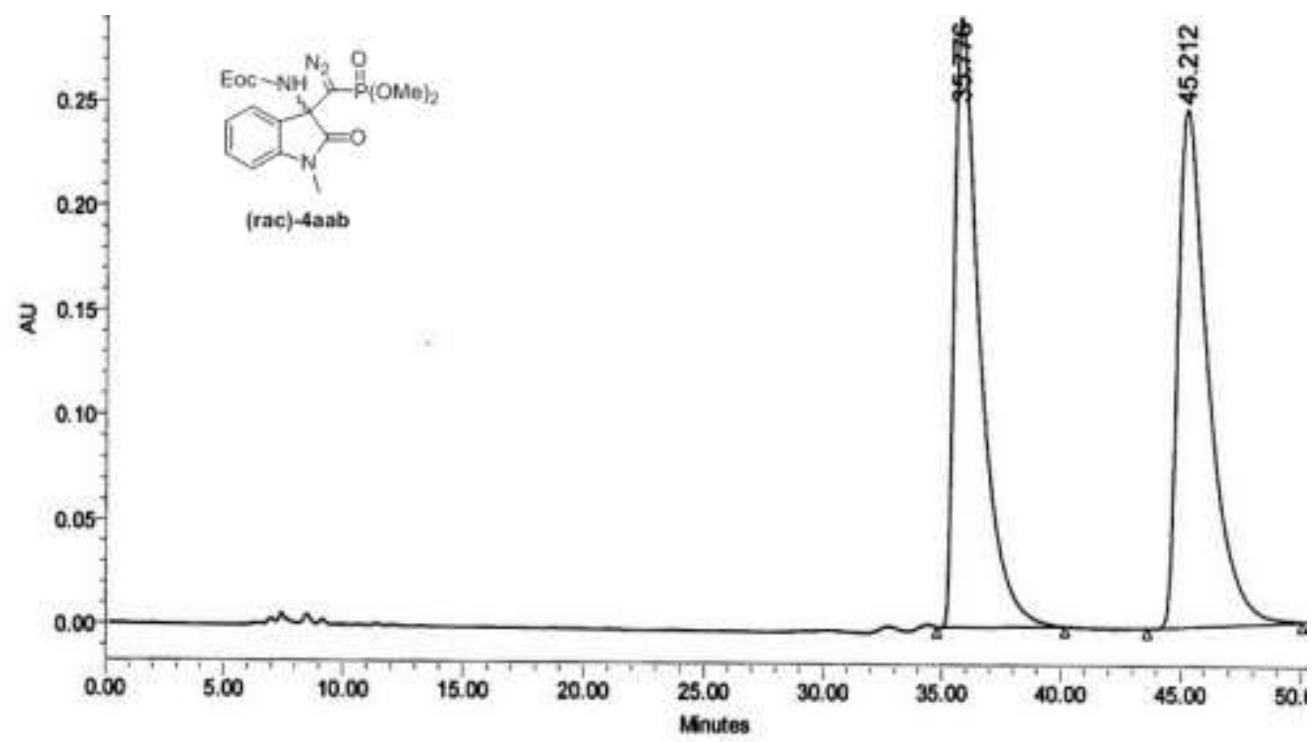

\begin{tabular}{|c|c|c|c|c|c|}
\hline & $\begin{array}{l}\text { RT } \\
\text { (min) }\end{array}$ & $\begin{array}{c}\text { Area } \\
\left.\text { ( }{ }^{\prime} V^{\prime} \text { 'sec }\right)\end{array}$ & $\%$ Area & $\begin{array}{l}\text { Height } \\
(\mu \mathrm{V})\end{array}$ & $\begin{array}{c}\% \\
\text { Height }\end{array}$ \\
\hline 1 & 35.776 & 21819267 & 49.95 & 292421 & 54.12 \\
\hline 2 & 45.212 & 21862559 & 50.05 & 247850 & 45.8 \\
\hline
\end{tabular}

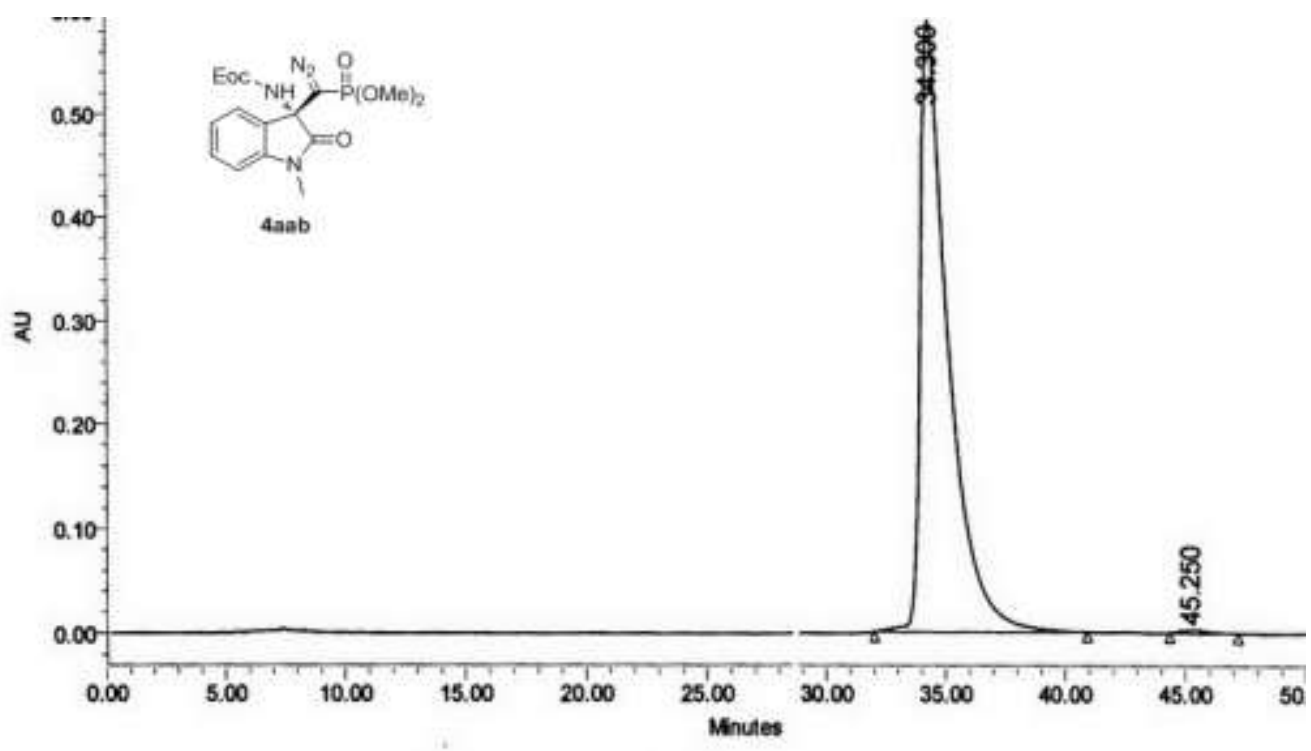

\begin{tabular}{|c|c|r|r|r|r|}
\hline & $\begin{array}{c}\text { Rा } \\
(\text { min })\end{array}$ & $\begin{array}{c}\text { Area } \\
(\mu \mathrm{V} \text { "sec })\end{array}$ & \% Area & $\begin{array}{c}\text { Height } \\
(\mu \mathrm{N})\end{array}$ & $\begin{array}{c}\% \\
\text { Height }\end{array}$ \\
\hline 1 & 34.300 & 48116299 & 99.51 & 588904 & 99.50 \\
\hline 2 & 45.250 & 236739 & 0.49 & 2932 & 0.50 \\
\hline
\end{tabular}




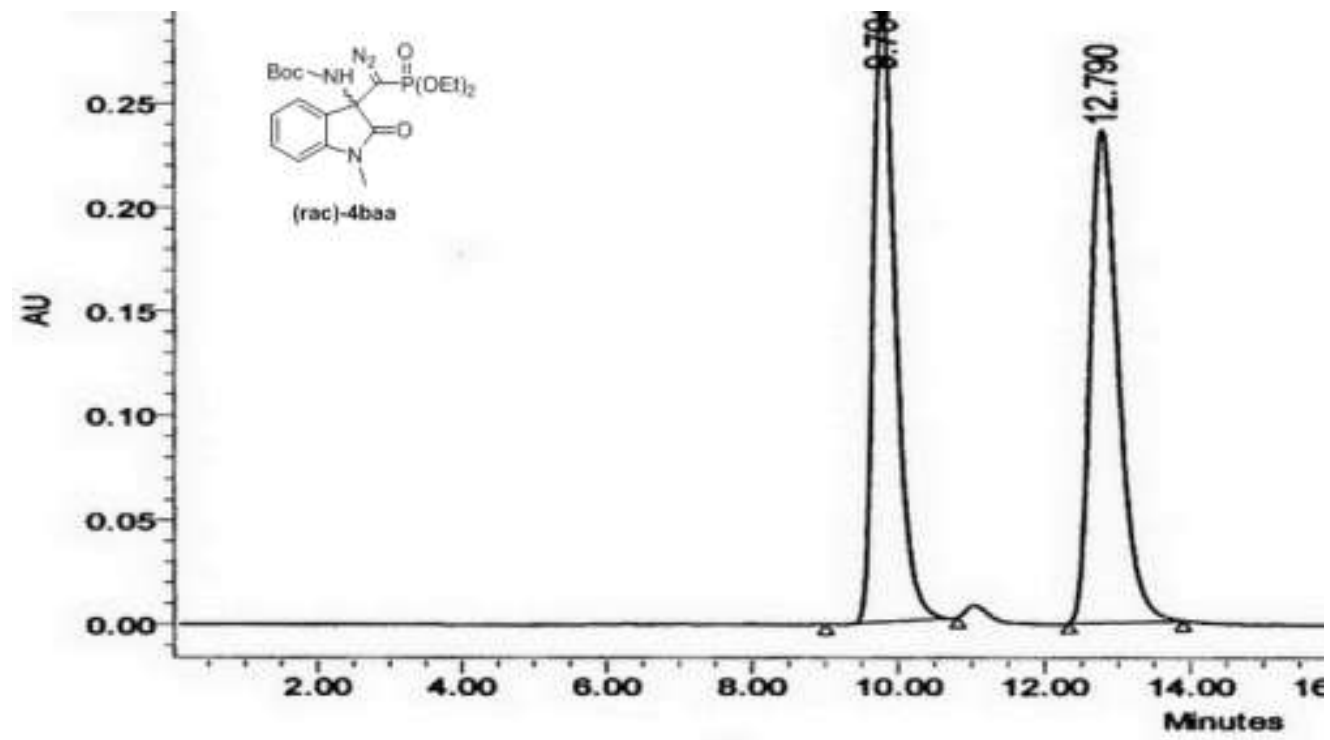

\begin{tabular}{|c|c|c|c|c|c|}
\hline & $\begin{array}{c}\text { RT } \\
(\text { min })\end{array}$ & $\begin{array}{c}\text { Area } \\
\left(\mu \mathrm{N}^{*} \text { sec }\right)\end{array}$ & $\%$ Area & $\begin{array}{c}\text { Height } \\
(\mu \mathrm{N})\end{array}$ & $\begin{array}{c}\% \\
\text { Height }\end{array}$ \\
\hline 1 & 9.794 & 6240972 & 49.76 & 300441 & 55.95 \\
\hline 2 & 12.790 & 6301402 & 50.24 & 236516 & 44.05 \\
\hline
\end{tabular}

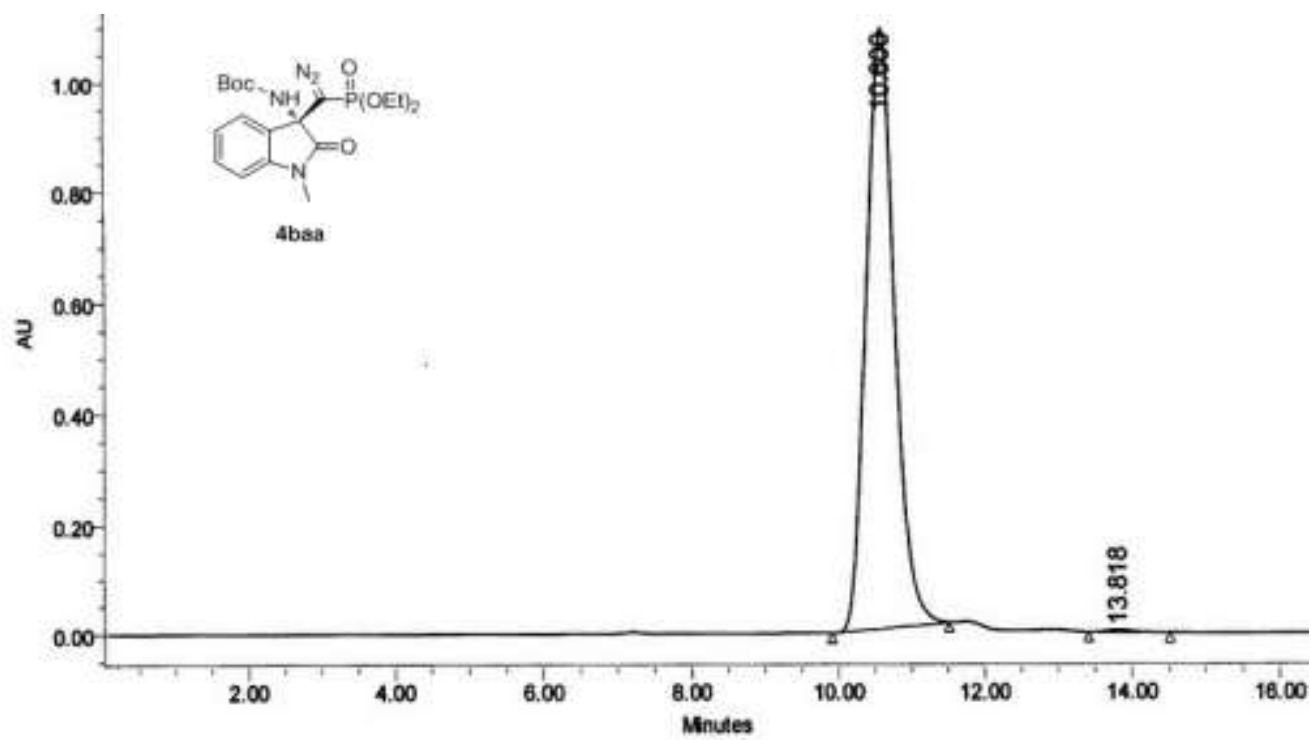

\begin{tabular}{|c|c|r|r|r|r|}
\hline & $\begin{array}{c}\text { RT } \\
(\mathrm{min})\end{array}$ & $\begin{array}{c}\text { Area } \\
(\mu \mathrm{N} \text { "sec })\end{array}$ & \% Area & $\begin{array}{c}\text { Height } \\
(\mu \mathrm{V})\end{array}$ & $\begin{array}{c}\% \\
\text { Height }\end{array}$ \\
\hline 1 & 10.600 & 29343868 & 99.67 & 1088091 & 99.62 \\
\hline 2 & 13.818 & 97910 & 0.33 & 4182 & 0.38 \\
\hline
\end{tabular}




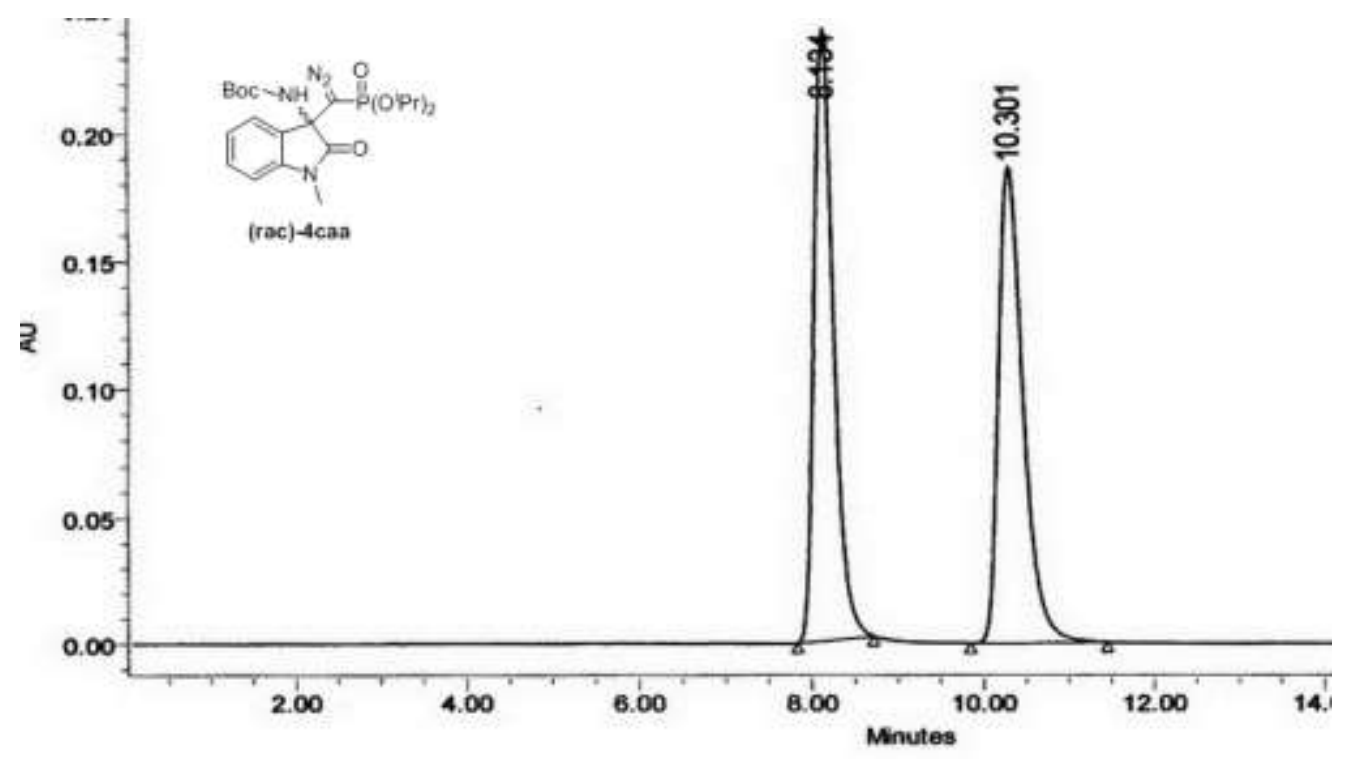

\begin{tabular}{|c|c|c|c|c|c|}
\hline & $\begin{array}{c}\text { RI } \\
(\min )\end{array}$ & $\begin{array}{c}\text { Area } \\
\left(\mu \mathrm{V}^{*} \mathrm{sec}\right)\end{array}$ & \% Area & $\begin{array}{c}\text { Height } \\
(\mu \mathrm{V})\end{array}$ & $\begin{array}{c}\% \\
\text { Height }\end{array}$ \\
\hline 1 & 8.134 & 3783507 & 50.20 & 240414 & 56.32 \\
\hline 2 & 10.301 & 3753575 & 49.80 & 186426 & 43.68 \\
\hline
\end{tabular}

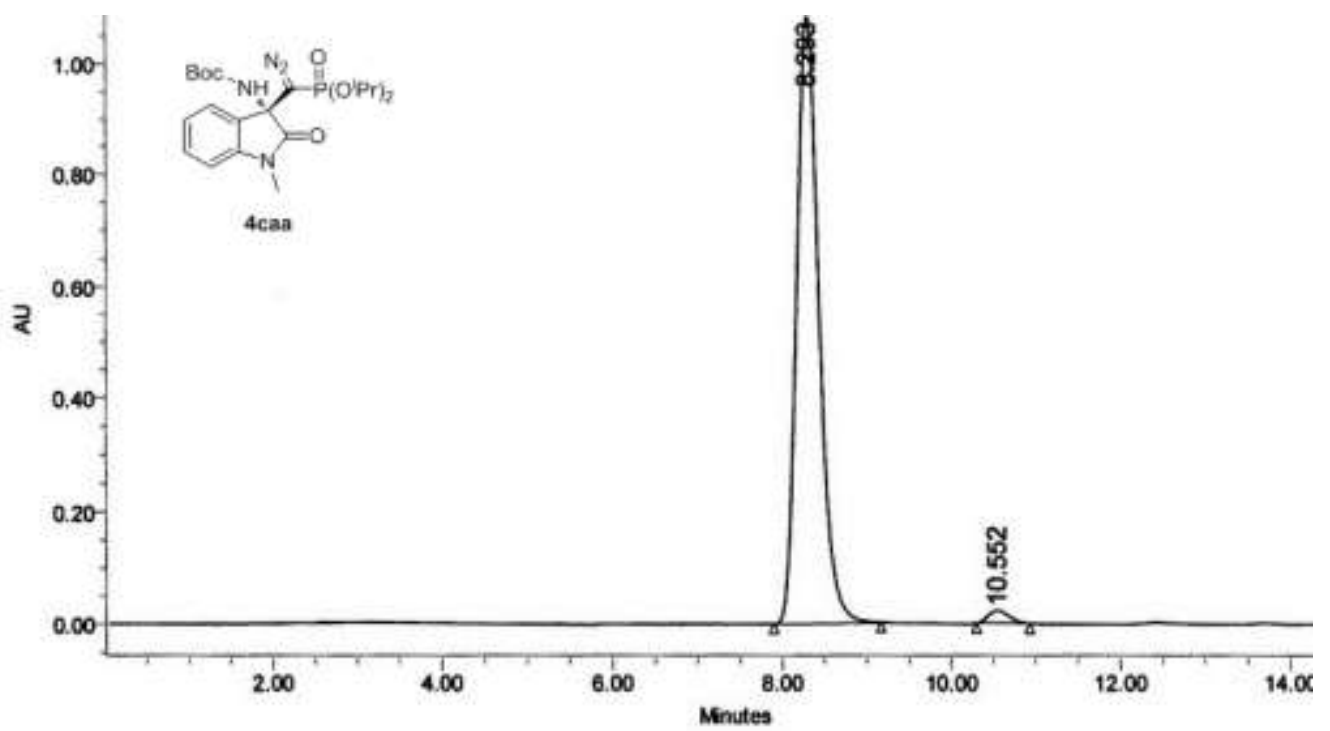

\begin{tabular}{|c|c|r|r|r|r|}
\hline & $\begin{array}{c}\text { RT } \\
(m i n)\end{array}$ & $\begin{array}{c}\text { Area } \\
(\mu \mathrm{N} / \mathrm{sec})\end{array}$ & $\%$ Area & $\begin{array}{c}\text { Height } \\
(\mu \mathrm{M})\end{array}$ & $\begin{array}{c}\% \\
\text { Height }\end{array}$ \\
\hline 1 & 8.293 & 19719456 & 98.03 & 1084847 & 97.96 \\
\hline 2 & 10.552 & 395385 & 1.97 & 22615 & 2.04 \\
\hline
\end{tabular}




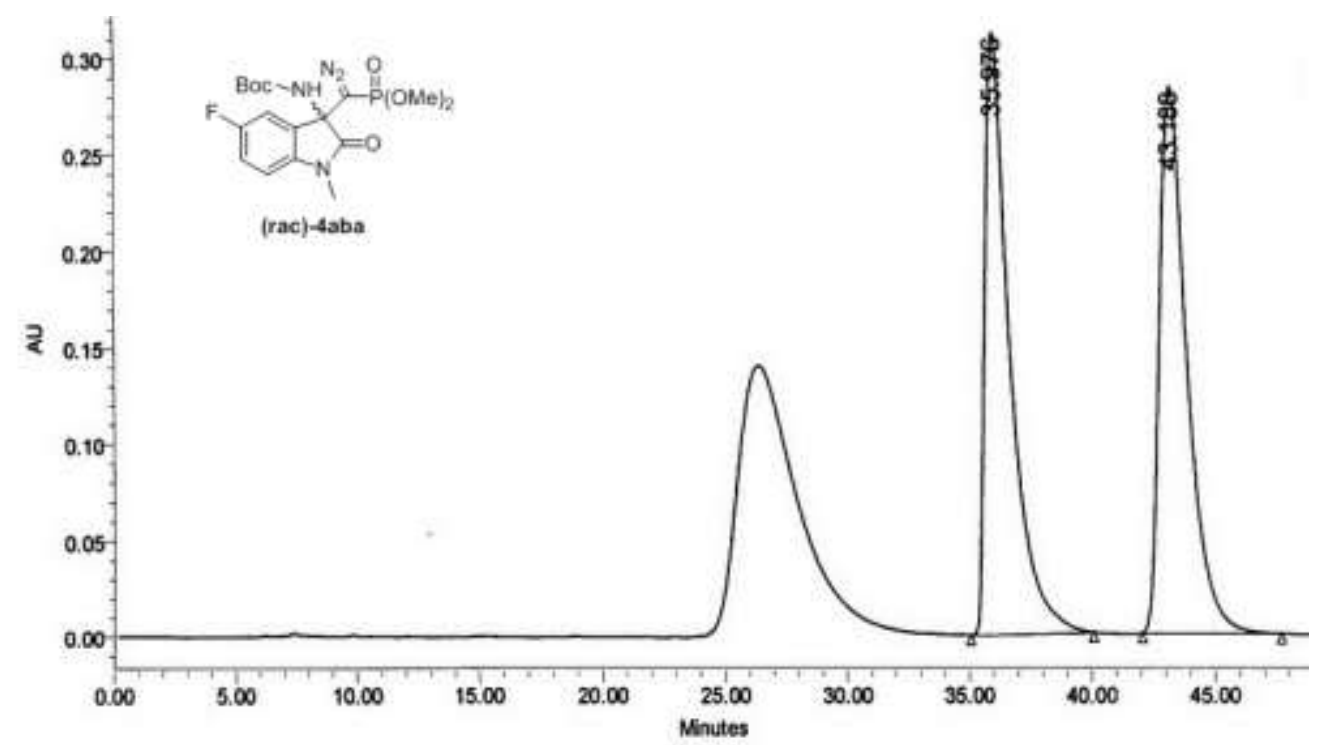

\begin{tabular}{|c|c|c|c|c|c|}
\hline & $\begin{array}{c}\text { RT } \\
(\mathrm{min})\end{array}$ & $\begin{array}{c}\text { Area } \\
(\mu \mathrm{N} \text { "sec) }\end{array}$ & \% Area & $\begin{array}{c}\text { Height } \\
(\mu \mathrm{M})\end{array}$ & $\begin{array}{c}\% \\
\text { Height }\end{array}$ \\
\hline 1 & 35.976 & 22064528 & 50.21 & 311886 & 52.41 \\
\hline 2 & 43.188 & 21879263 & 49.79 & 283183 & 47.59 \\
\hline
\end{tabular}

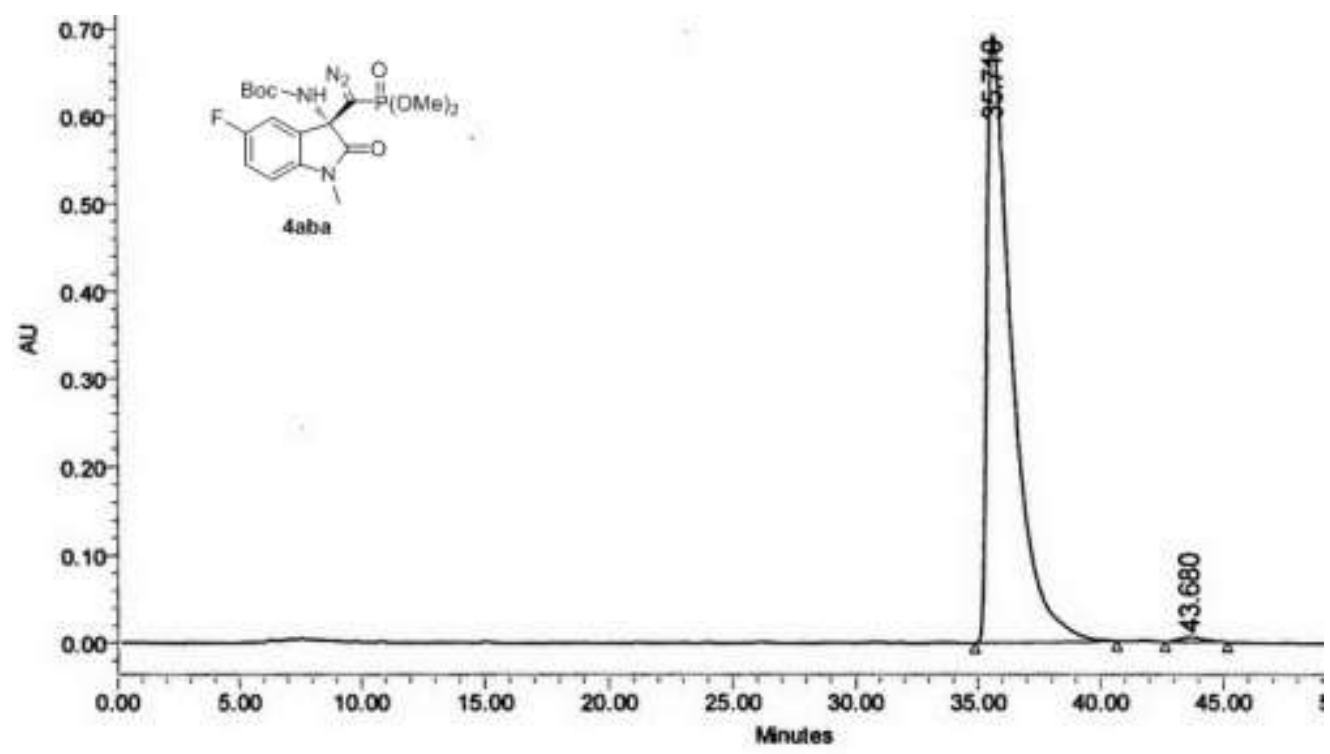

\begin{tabular}{|c|c|r|r|r|r|}
\hline & $\begin{array}{c}\text { Rा } \\
(\mathrm{min})\end{array}$ & $\begin{array}{c}\text { Area } \\
(\mu \mathrm{N} \text { *sec })\end{array}$ & \% Area & \multicolumn{1}{|c|}{$\begin{array}{c}\text { Height } \\
(\mu \mathrm{N})\end{array}$} & $\begin{array}{c}\% \\
\text { Height }\end{array}$ \\
\hline 1 & 35.710 & 49331648 & 99.27 & 692940 & 99.26 \\
\hline 2 & 43.680 & 364845 & 0.73 & 5163 & 0.74 \\
\hline
\end{tabular}




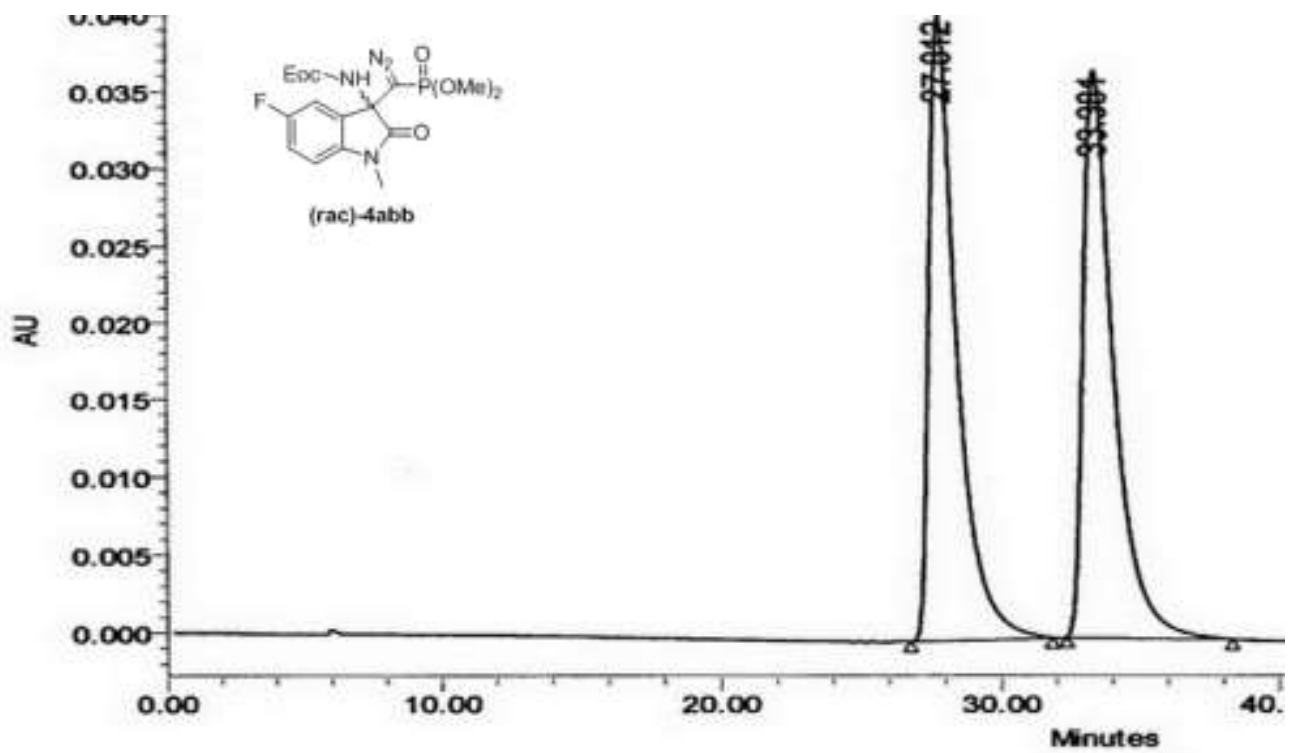

\begin{tabular}{|c|c|c|r|r|r|}
\hline & $\begin{array}{c}\text { RT } \\
(\min )\end{array}$ & $\begin{array}{c}\text { Area } \\
\left(\mu \mathrm{V}^{*} \mathrm{sec}\right)\end{array}$ & \% Area & $\begin{array}{c}\text { Height } \\
(\mu \mathrm{V})\end{array}$ & $\begin{array}{c}\% \\
\text { Height }\end{array}$ \\
\hline 1 & 27.812 & 2775033 & 50.03 & 40370 & 52.41 \\
\hline 2 & 33.381 & 2772245 & 49.97 & 36656 & 47.59 \\
\hline
\end{tabular}

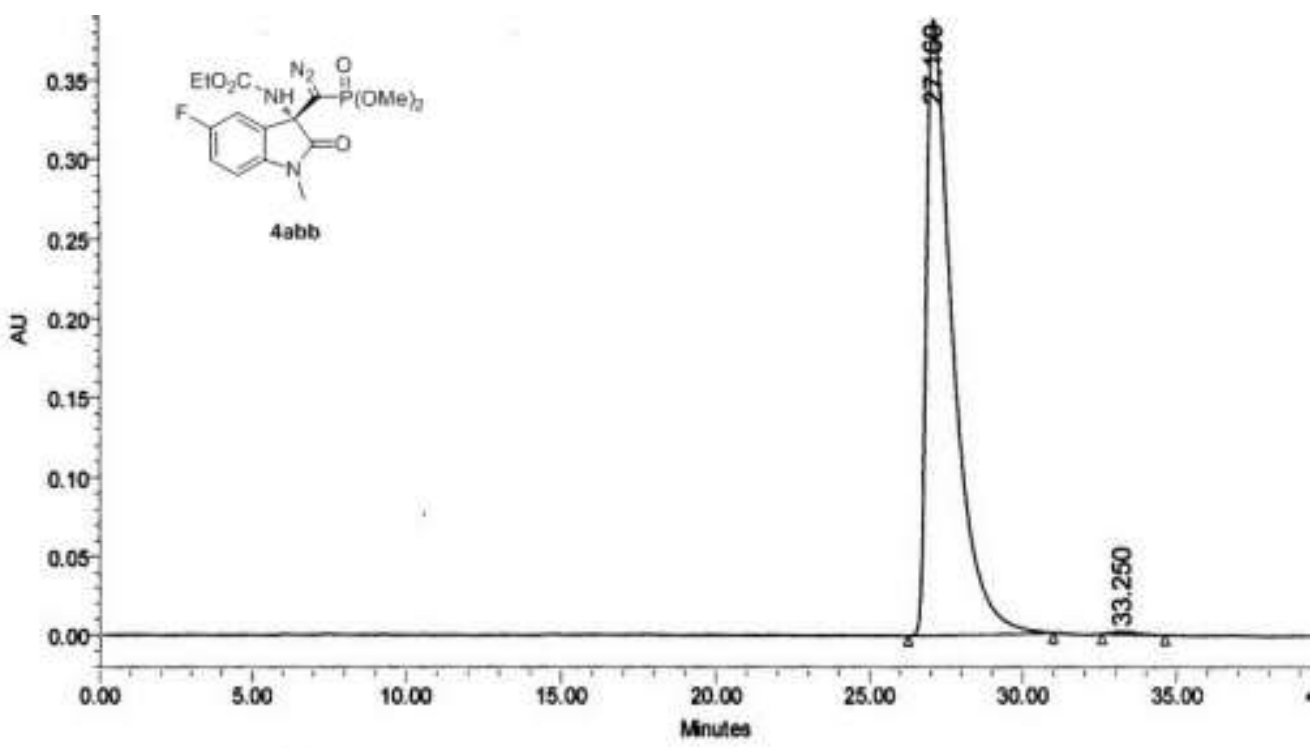

\begin{tabular}{|r|c|r|r|r|r|}
\hline & $\begin{array}{c}\text { RT } \\
(\mathrm{min})\end{array}$ & $\begin{array}{c}\text { Area } \\
(\mu \mathrm{N} * \mathrm{sec})\end{array}$ & \% Area & $\begin{array}{c}\text { Height } \\
(\mu \mathrm{N})\end{array}$ & $\begin{array}{c}\% \\
\text { Height }\end{array}$ \\
\hline 1 & 27.160 & 23335266 & 99.58 & 388496 & 99.57 \\
\hline 2 & 33.250 & 98989 & 0.42 & 1668 & 0.43 \\
\hline
\end{tabular}




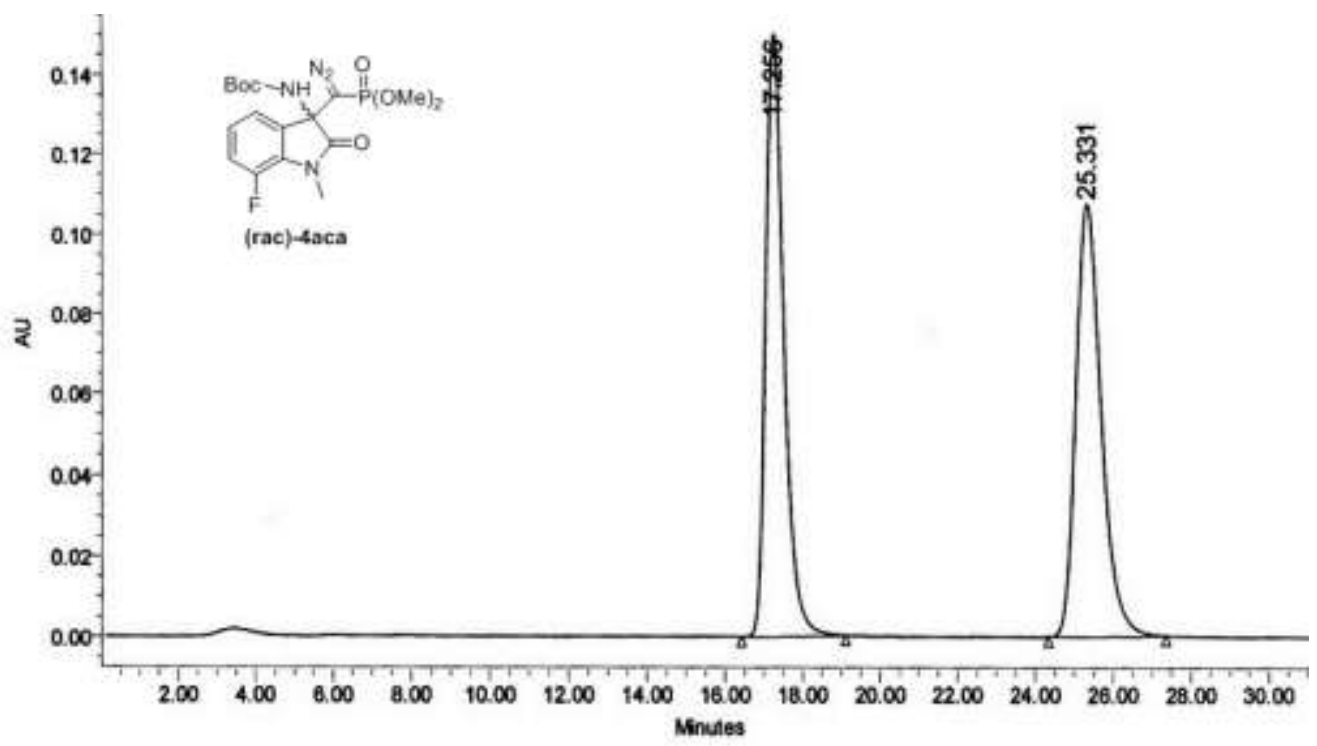

\begin{tabular}{|c|c|c|c|c|c|}
\hline & $\begin{array}{c}\text { Rा } \\
(\mathrm{min})\end{array}$ & $\begin{array}{c}\text { Area } \\
\left(\mu \mathrm{N}^{*} \text { sec }\right)\end{array}$ & $\%$ Area & $\begin{array}{c}\text { Height } \\
(\mu \mathrm{N})\end{array}$ & $\begin{array}{c}\% \\
\text { Height }\end{array}$ \\
\hline 1 & 17.256 & 4875632 & 50.12 & 150363 & 58.27 \\
\hline 2 & 25.331 & 4852518 & 49.88 & 107698 & 41.73 \\
\hline
\end{tabular}

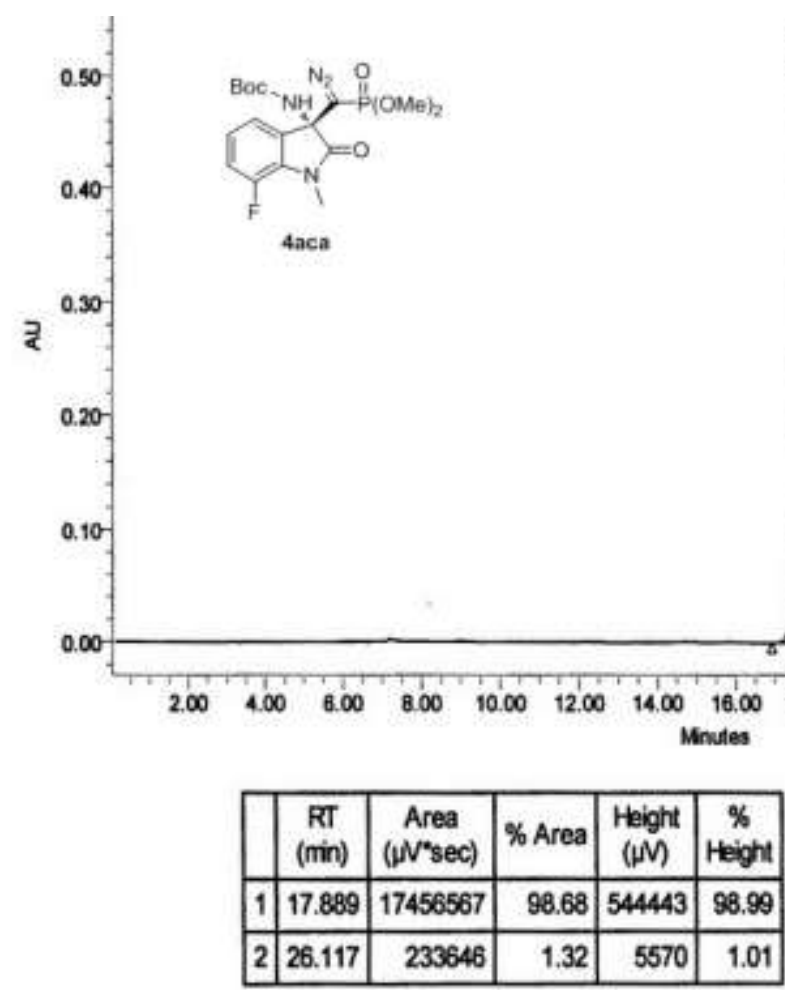




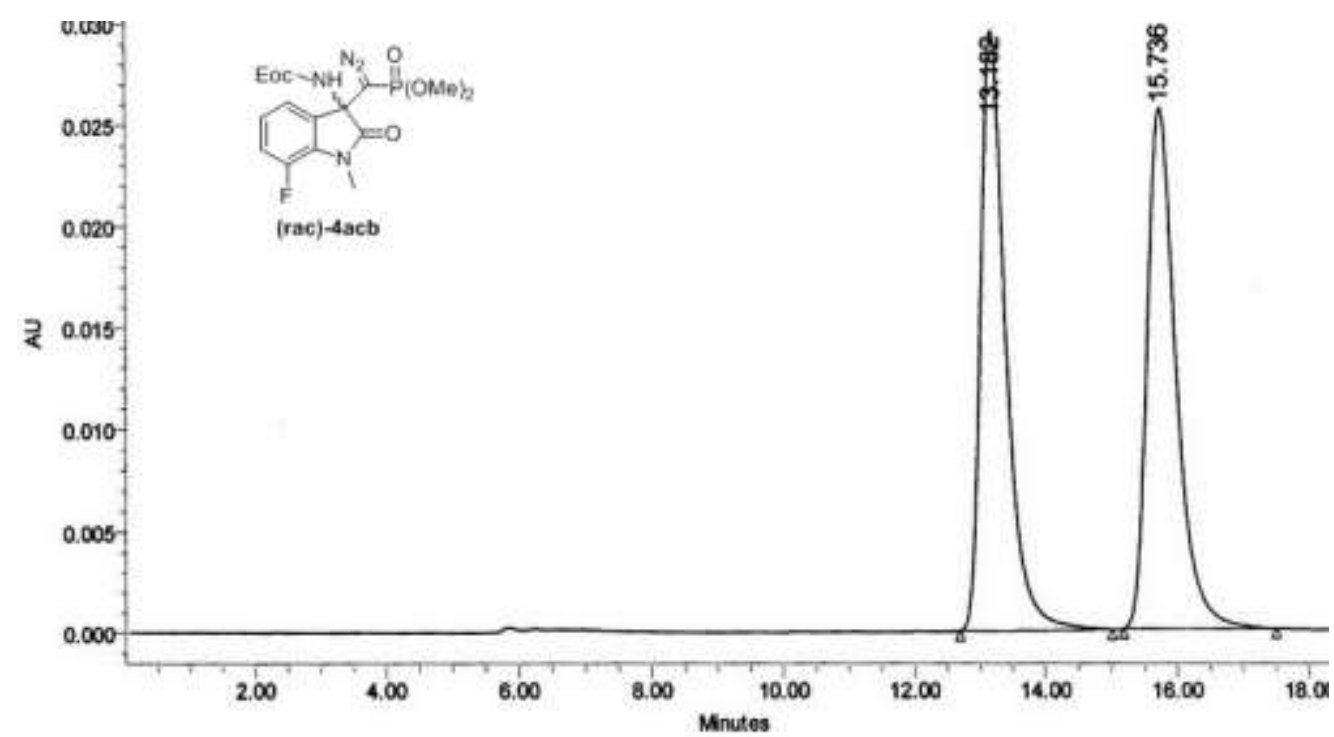

\begin{tabular}{|c|c|c|c|c|c|}
\hline & $\begin{array}{c}\text { RT } \\
(\mathrm{min})\end{array}$ & $\begin{array}{c}\text { Area } \\
(\mu \mathrm{V} \text { "sec })\end{array}$ & \% Area & $\begin{array}{c}\text { Height } \\
(\mu \mathrm{N})\end{array}$ & $\begin{array}{c}\% \\
\text { Height }\end{array}$ \\
\hline 1 & 13.182 & 783224 & 50.21 & 29533 & 53.53 \\
\hline 2 & 15.736 & 776525 & 49.79 & 25643 & 46.47 \\
\hline
\end{tabular}

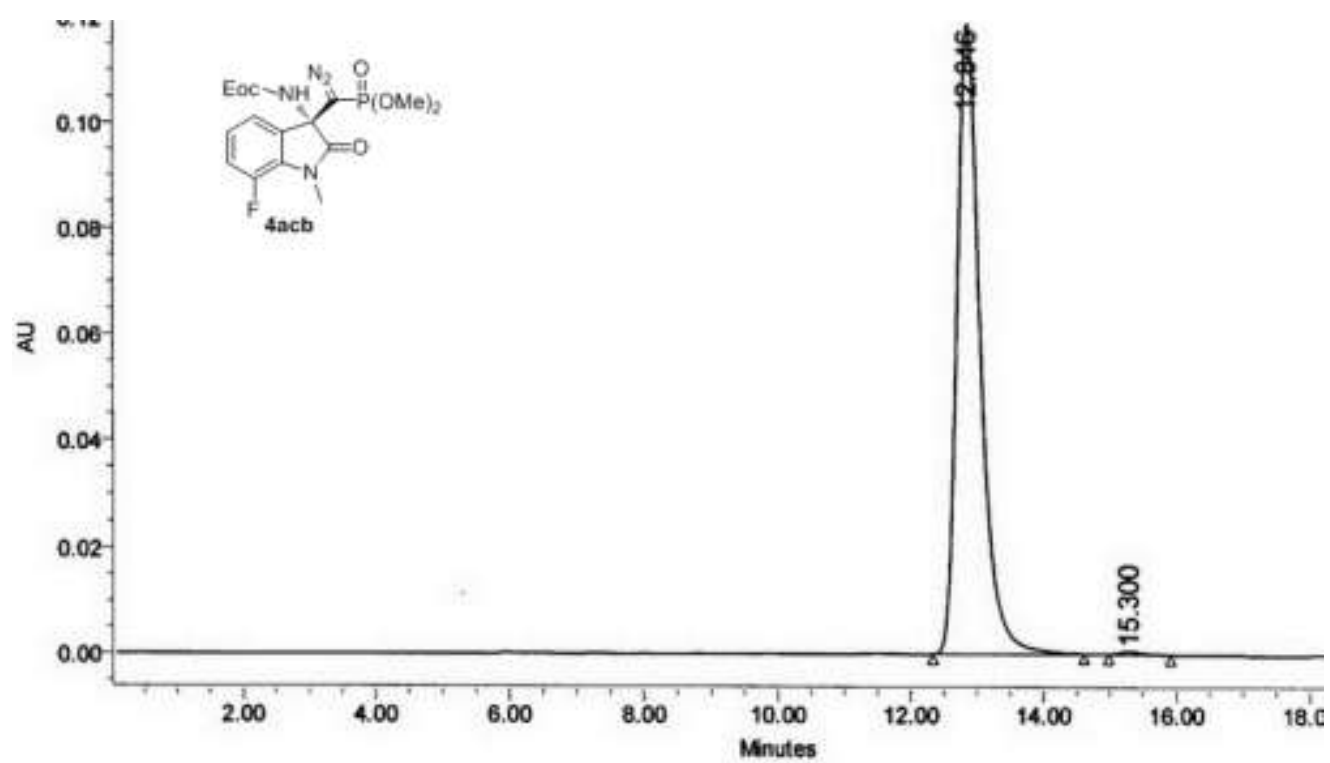

\begin{tabular}{|c|c|r|r|r|r|}
\hline & $\begin{array}{c}\text { Rा } \\
(\mathrm{min})\end{array}$ & $\begin{array}{c}\text { Area } \\
(\mu \mathrm{V} * \mathrm{sec})\end{array}$ & $\%$ Area & $\begin{array}{c}\text { Height } \\
(\mu \mathrm{V})\end{array}$ & $\begin{array}{c}\% \\
\text { Height }\end{array}$ \\
\hline 1 & 12.846 & 2916656 & 99.54 & 118591 & 99.55 \\
\hline 2 & 15.300 & 13354 & 0.46 & 540 & 0.45 \\
\hline
\end{tabular}




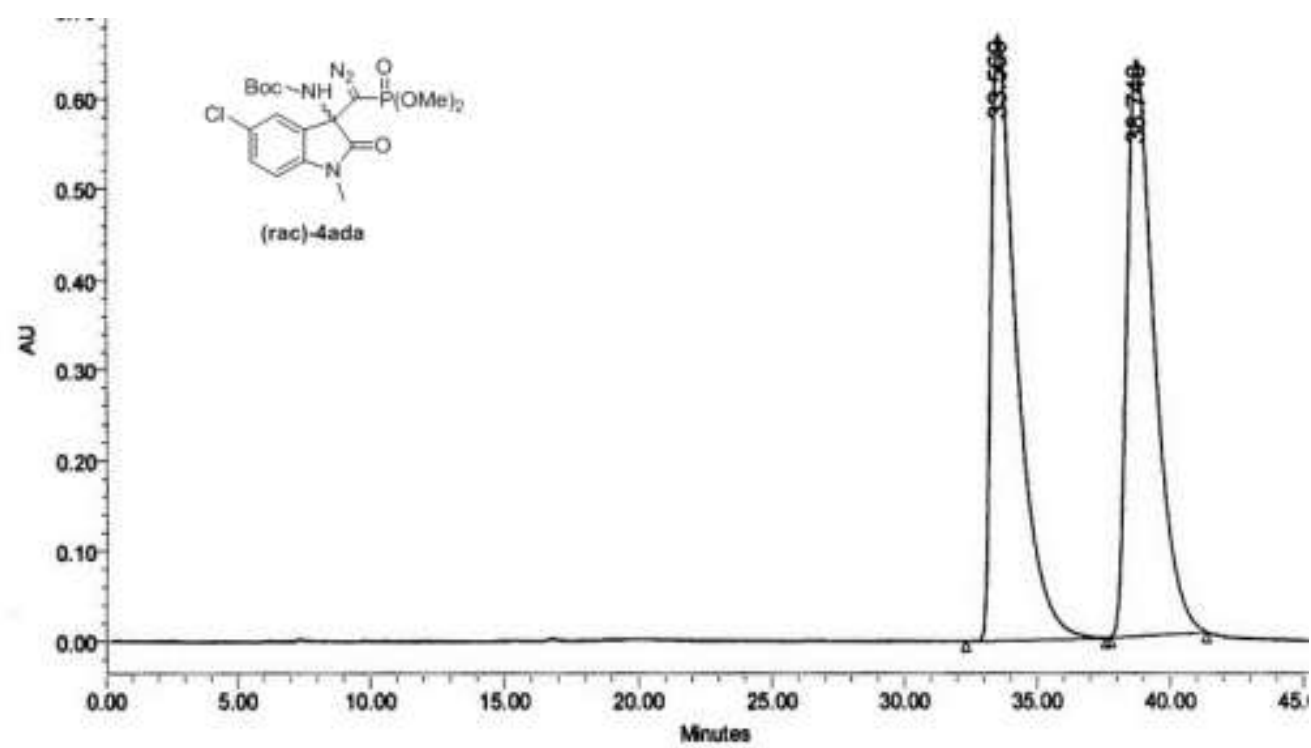

\begin{tabular}{|c|c|c|c|c|c|}
\hline & $\begin{array}{c}\text { RT } \\
(\mathrm{min})\end{array}$ & $\begin{array}{c}\text { Area } \\
(\mu \mathrm{V} \text { sec })\end{array}$ & \% Area & $\begin{array}{c}\text { Height } \\
(\mu \mathrm{V})\end{array}$ & $\begin{array}{c}\% \\
\text { Height }\end{array}$ \\
\hline 1 & 33.569 & 44949673 & 49.80 & 670093 & 51.26 \\
\hline 2 & 38.748 & 45314667 & 50.20 & 637237 & 48.74 \\
\hline
\end{tabular}

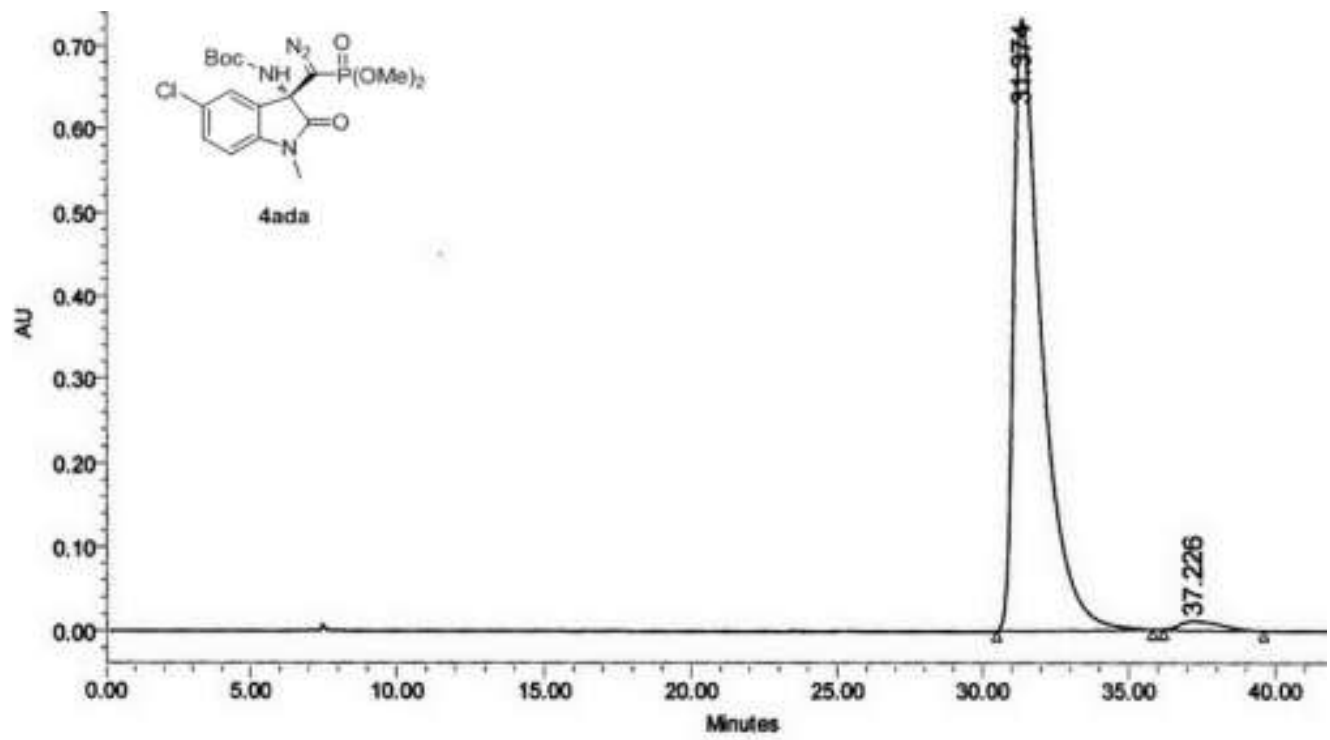

\begin{tabular}{|c|c|c|r|r|r|}
\hline & $\begin{array}{c}\mathrm{R} \\
(\mathrm{min})\end{array}$ & $\begin{array}{c}\text { Area } \\
(\mu \mathrm{N} \text { "sec })\end{array}$ & \% Area & $\begin{array}{c}\text { Height } \\
(\mu \mathrm{V})\end{array}$ & $\begin{array}{c}\% \\
\text { Height }\end{array}$ \\
\hline 1 & 31.374 & 48756758 & 98.00 & 732649 & 98.57 \\
\hline 2 & 37.226 & 993894 & 2.00 & 10592 & 1.43 \\
\hline
\end{tabular}




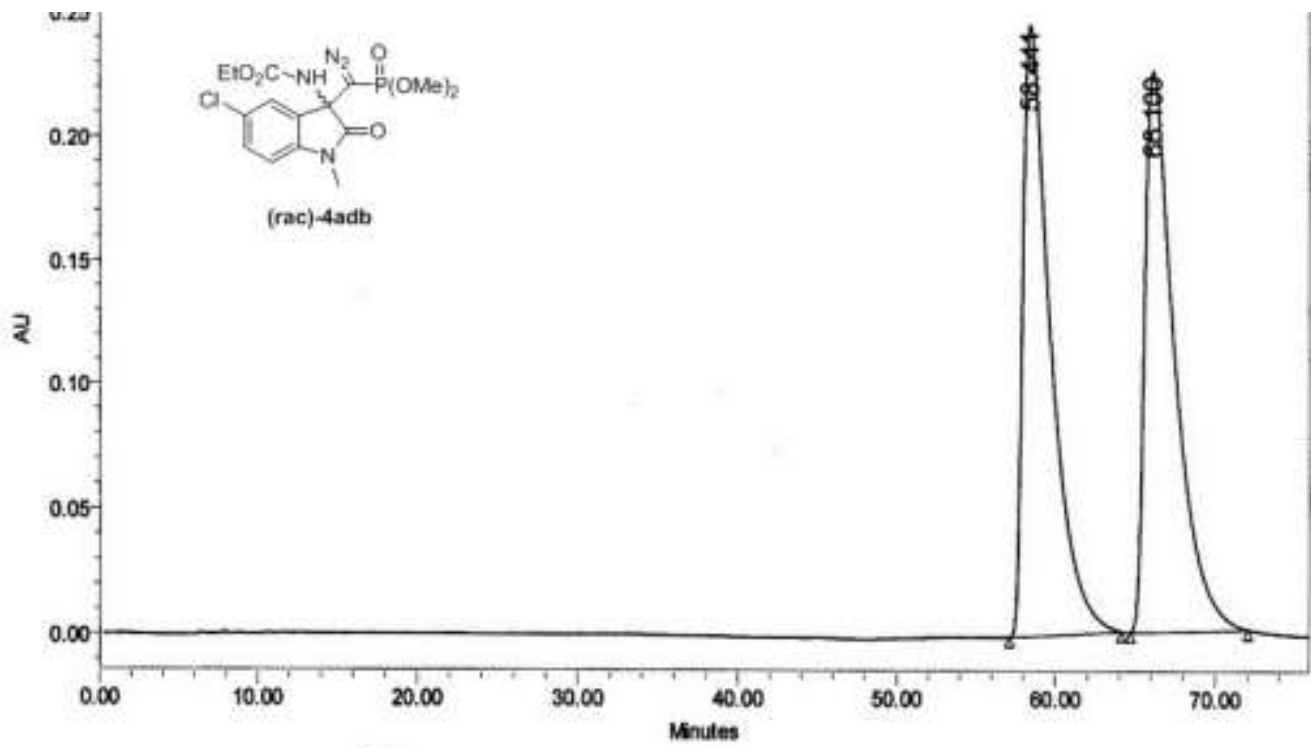

\begin{tabular}{|c|c|c|c|c|c|}
\hline & $\begin{array}{c}\text { RT } \\
(\mathrm{min})\end{array}$ & $\begin{array}{c}\text { Area } \\
(\mu \mathrm{V} \text { 'sec })\end{array}$ & $\%$ Area & $\begin{array}{c}\text { Height } \\
(\mu \mathrm{N})\end{array}$ & $\begin{array}{c}\% \\
\text { Height }\end{array}$ \\
\hline 1 & 58.441 & 29378472 & 50.30 & 245500 & 52.16 \\
\hline 2 & 66.100 & 29029581 & 49.70 & 225133 & 47.84 \\
\hline
\end{tabular}

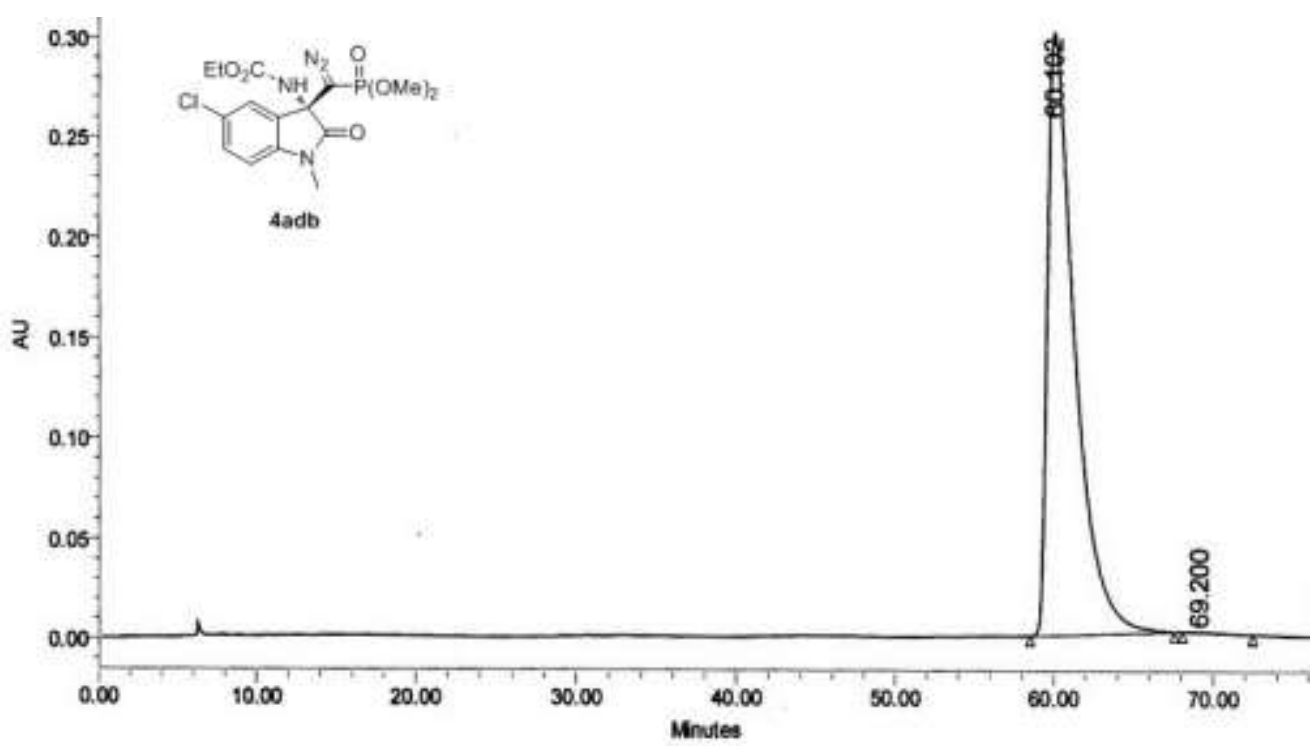

\begin{tabular}{|c|c|r|r|r|r|}
\hline & $\begin{array}{c}\text { RT } \\
(\mathrm{min})\end{array}$ & $\begin{array}{c}\text { Area } \\
(\mu \mathrm{N} / \mathrm{sec})\end{array}$ & \% Area & $\begin{array}{c}\text { Height } \\
(\mu \mathrm{M})\end{array}$ & $\begin{array}{c}\% \\
\text { Height }\end{array}$ \\
\hline 1 & 60.102 & 35556954 & 99.86 & 299808 & 99.87 \\
\hline 2 & 69.200 & 50742 & 0.14 & 399 & 0.13 \\
\hline
\end{tabular}



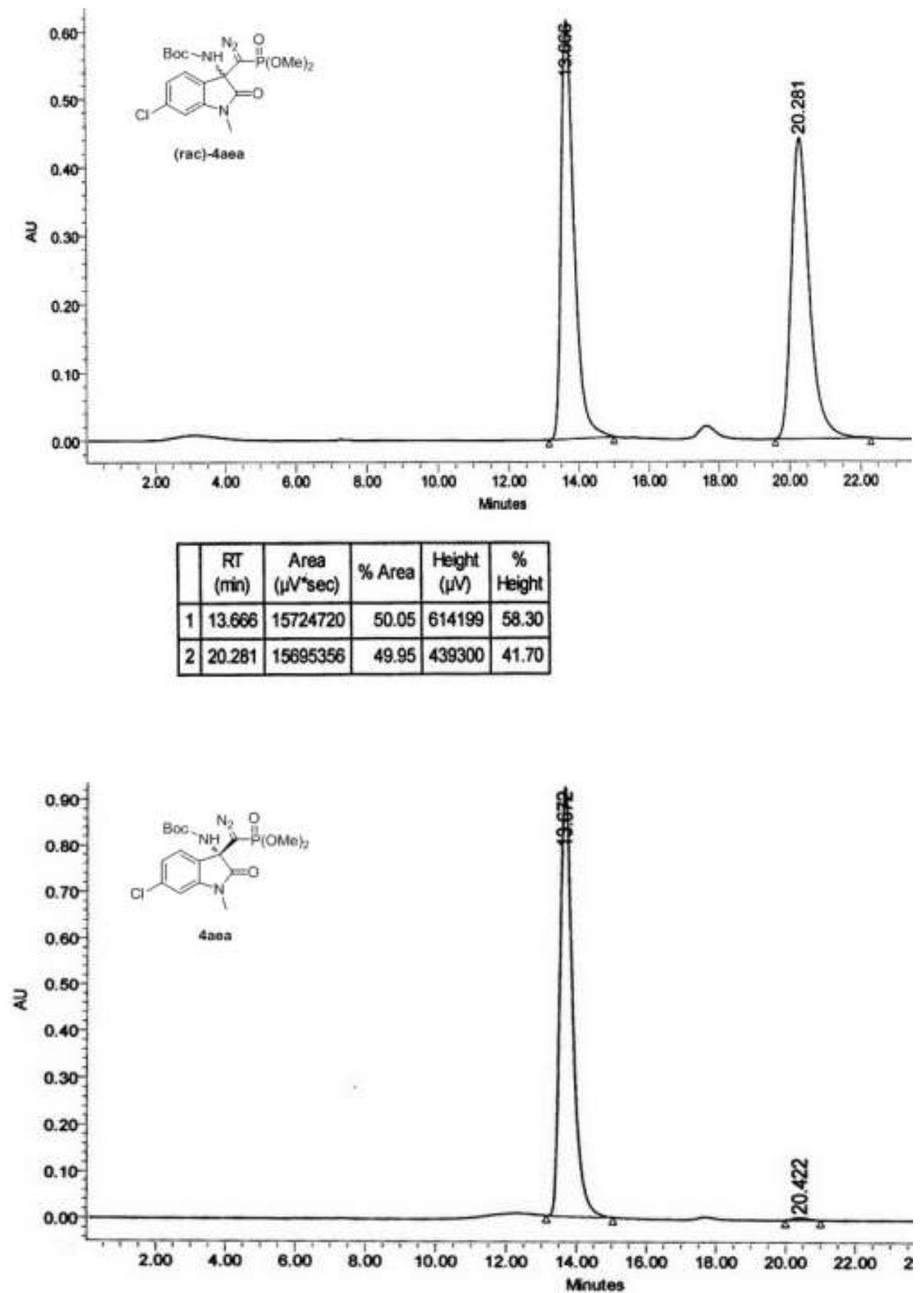

\begin{tabular}{|c|c|r|r|r|r|}
\hline & $\begin{array}{c}\text { RT } \\
(\mathrm{min})\end{array}$ & $\begin{array}{c}\text { Area } \\
(\mu \mathrm{N} \text { "sec })\end{array}$ & $\%$ Area & $\begin{array}{c}\text { Height } \\
(\mu \mathrm{V})\end{array}$ & $\begin{array}{c}\% \\
\text { Height }\end{array}$ \\
\hline 1 & 13.672 & 23241654 & 99.32 & 926373 & 99.44 \\
\hline 2 & 20.422 & 160075 & 0.68 & 5263 & 0.56 \\
\hline
\end{tabular}




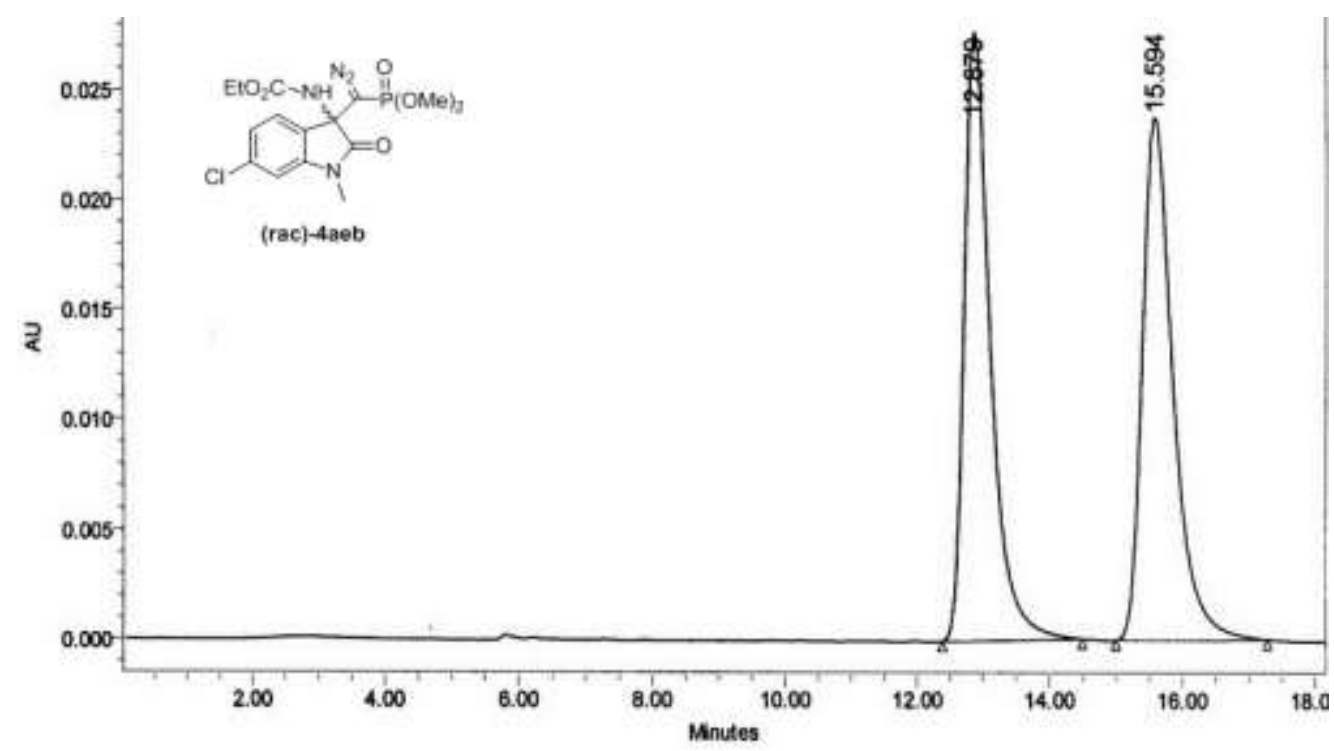

\begin{tabular}{|c|c|c|c|c|c|}
\hline & $\begin{array}{c}\text { RT } \\
(\min )\end{array}$ & $\begin{array}{c}\text { Area } \\
\left(\mu \mathrm{V}^{*} \mathrm{sec}\right)\end{array}$ & \% Area & $\begin{array}{c}\text { Height } \\
(\mu \mathrm{V})\end{array}$ & $\begin{array}{c}\% \\
\text { Height }\end{array}$ \\
\hline 1 & 12.879 & 762911 & 50.04 & 27617 & 53.81 \\
\hline 2 & 15.594 & 761718 & 49.96 & 23702 & 46.19 \\
\hline
\end{tabular}

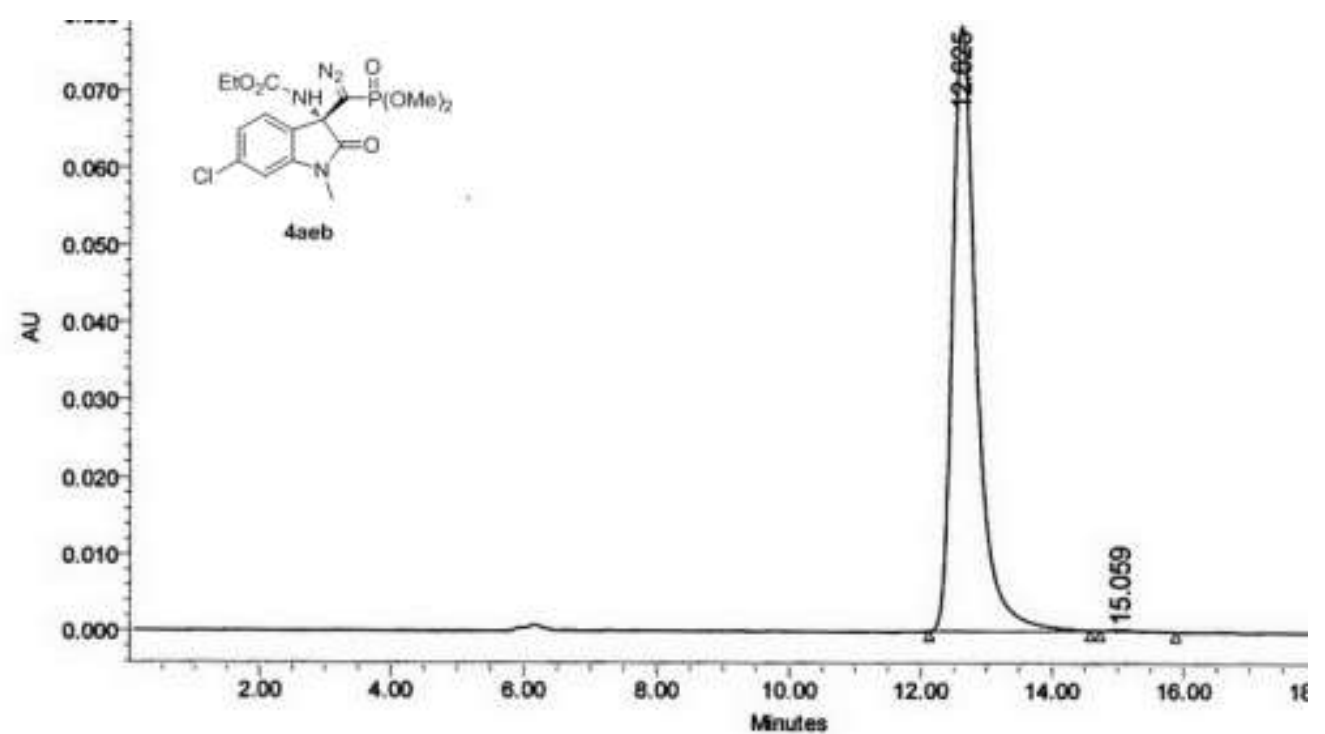

\begin{tabular}{|r|c|r|r|r|r|}
\hline & $\begin{array}{c}\text { RT } \\
(\mathrm{min})\end{array}$ & $\begin{array}{c}\text { Area } \\
\left(\mu \mathrm{N}^{*} \mathrm{sec}\right)\end{array}$ & $\%$ Area & $\begin{array}{c}\text { Height } \\
(\mu \mathrm{N})\end{array}$ & $\begin{array}{c}\% \\
\text { Height }\end{array}$ \\
\hline 1 & 12.625 & 2000287 & 99.70 & 78686 & 99.74 \\
\hline 2 & 15.059 & 6005 & 0.30 & 204 & 0.26 \\
\hline
\end{tabular}




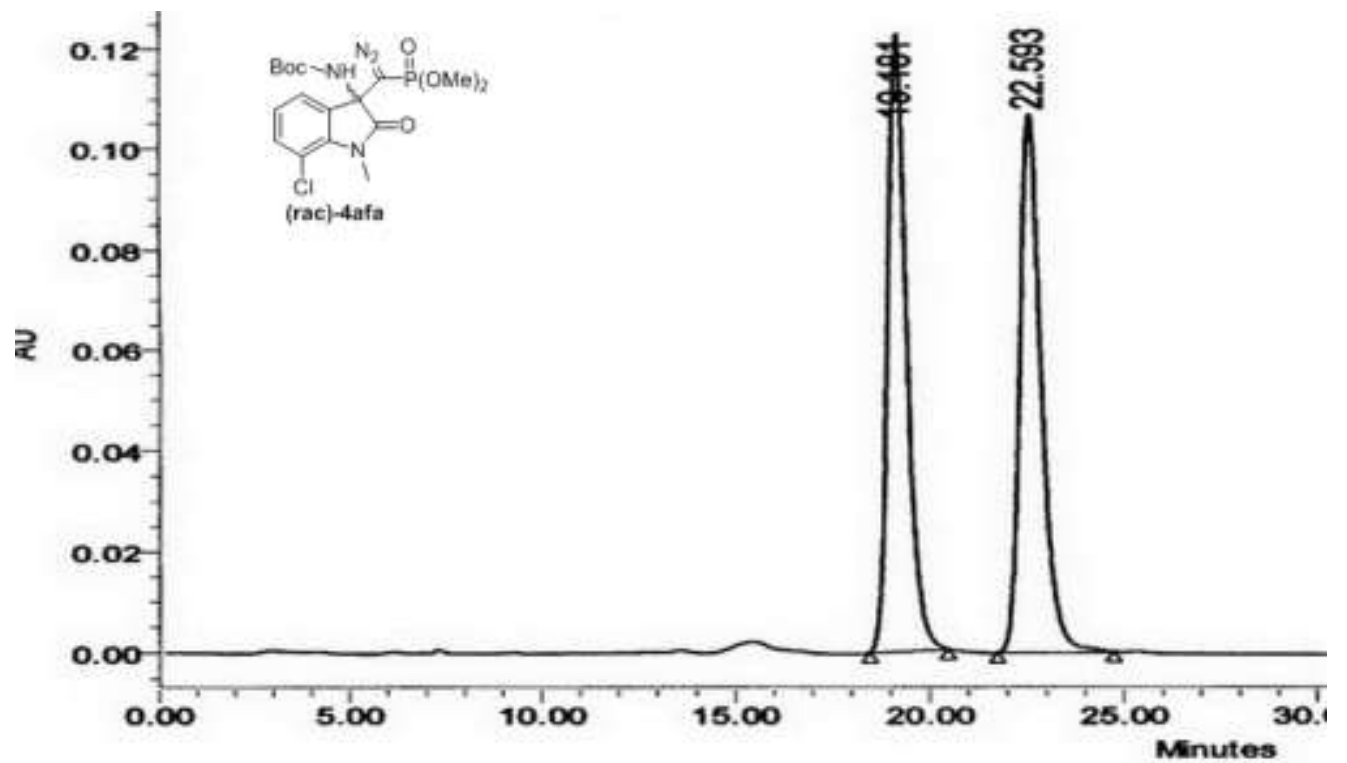

\begin{tabular}{|c|c|c|r|c|c|}
\hline & $\begin{array}{c}\text { RT } \\
(\mathrm{min})\end{array}$ & $\begin{array}{c}\text { Area } \\
\left(\mu v^{2} \text { sec) }\right.\end{array}$ & \% Area & $\begin{array}{c}\text { Height } \\
(\mu \mathrm{V})\end{array}$ & $\begin{array}{c}\% \\
\text { Height }\end{array}$ \\
\hline 1 & 19.181 & 4145456 & 50.34 & 122825 & 53.47 \\
\hline 2 & 22.593 & 4089060 & 49.66 & 106878 & 46.53
\end{tabular}

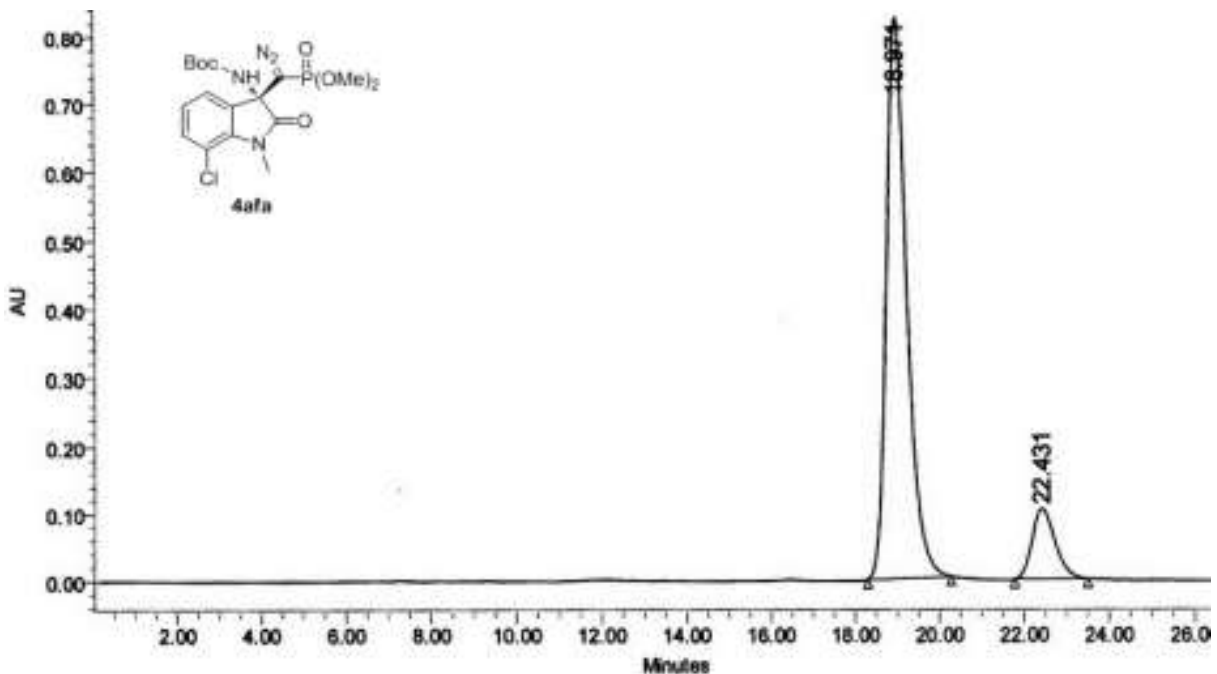

\begin{tabular}{|c|c|c|c|c|c|}
\hline & $\begin{array}{c}\text { RT } \\
(\mathrm{min})\end{array}$ & $\begin{array}{c}\text { Area } \\
\left(\mu \mathrm{N}^{\prime} \mathrm{sec}\right)\end{array}$ & \% Area & $\begin{array}{c}\text { Height } \\
(\mu \mathrm{V})\end{array}$ & $\begin{array}{c}\% \\
\text { Height }\end{array}$ \\
\hline 1 & 18.971 & 27644036 & 87.65 & 824880 & 88.72 \\
\hline 2 & 22.431 & 3896641 & 12.35 & 104836 & 11.28 \\
\hline
\end{tabular}




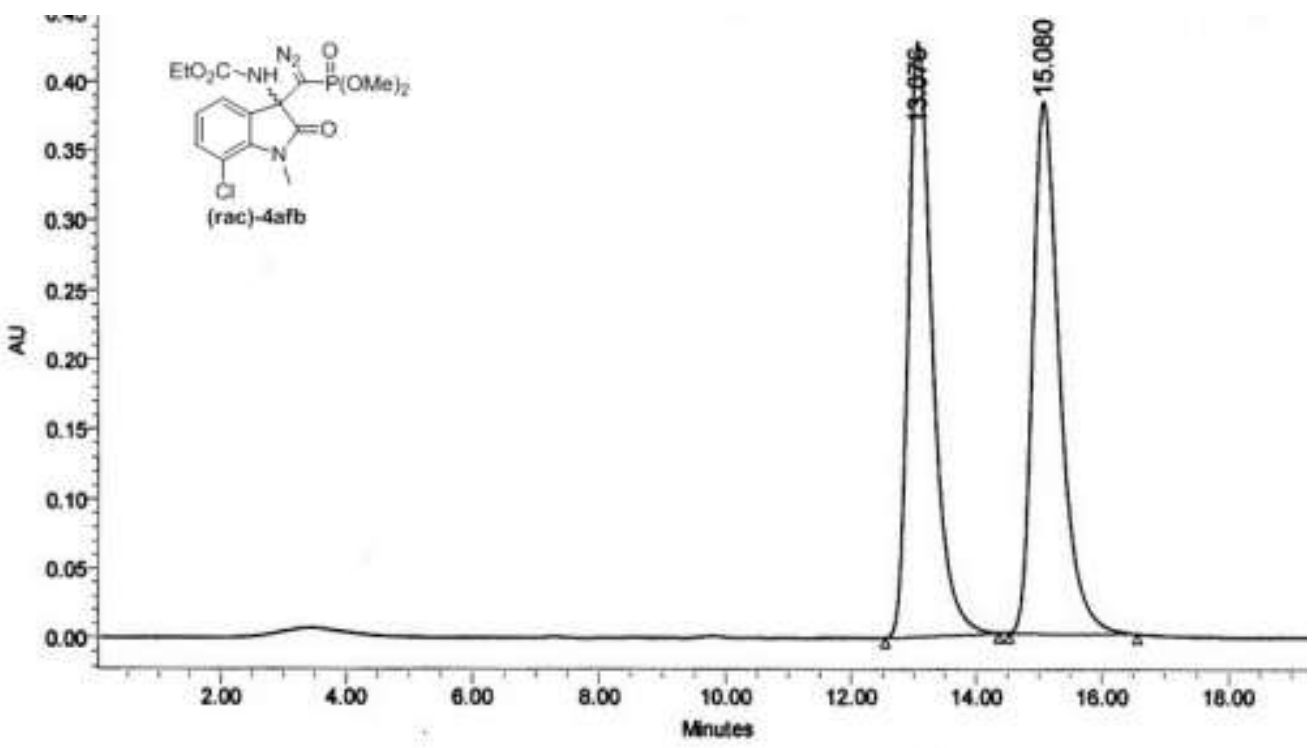

\begin{tabular}{|c|c|c|c|c|c|}
\hline & $\begin{array}{c}R T \\
(\min )\end{array}$ & $\begin{array}{c}\text { Area } \\
(\mu \mathrm{V} \text { sec })\end{array}$ & \% Area & $\begin{array}{c}\text { Height } \\
(\mu \mathrm{V})\end{array}$ & $\begin{array}{c}\% \\
\text { Height }\end{array}$ \\
\hline 1 & 13.076 & 11451955 & 50.17 & 427794 & 52.82 \\
\hline 2 & 15.080 & 11375210 & 49.83 & 382143 & $\mathbf{4 7 . 1 8}$ \\
\hline
\end{tabular}

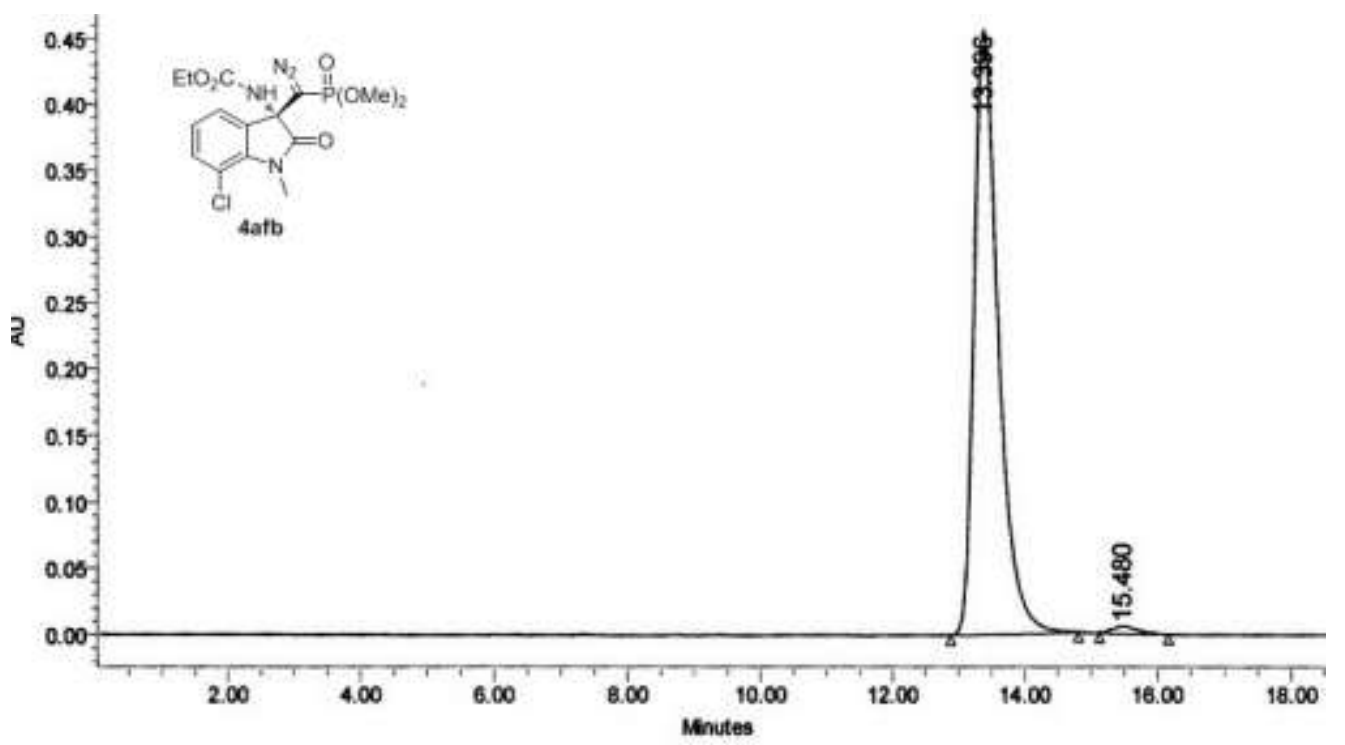

\begin{tabular}{|c|c|c|r|r|r|}
\hline & $\begin{array}{c}R T \\
(\mathrm{~min})\end{array}$ & $\begin{array}{c}\text { Area } \\
\left(\mu \mathrm{V}^{2} \mathrm{sec}\right)\end{array}$ & \% Area & $\begin{array}{c}\text { Height } \\
(\mu \mathrm{N})\end{array}$ & $\begin{array}{c}\text { \% } \\
\text { Height }\end{array}$ \\
\hline 1 & 13.396 & 11649921 & 98.82 & 457415 & 98.91 \\
\hline 2 & 15.480 & 139143 & 1.18 & 5064 & 1.09 \\
\hline
\end{tabular}




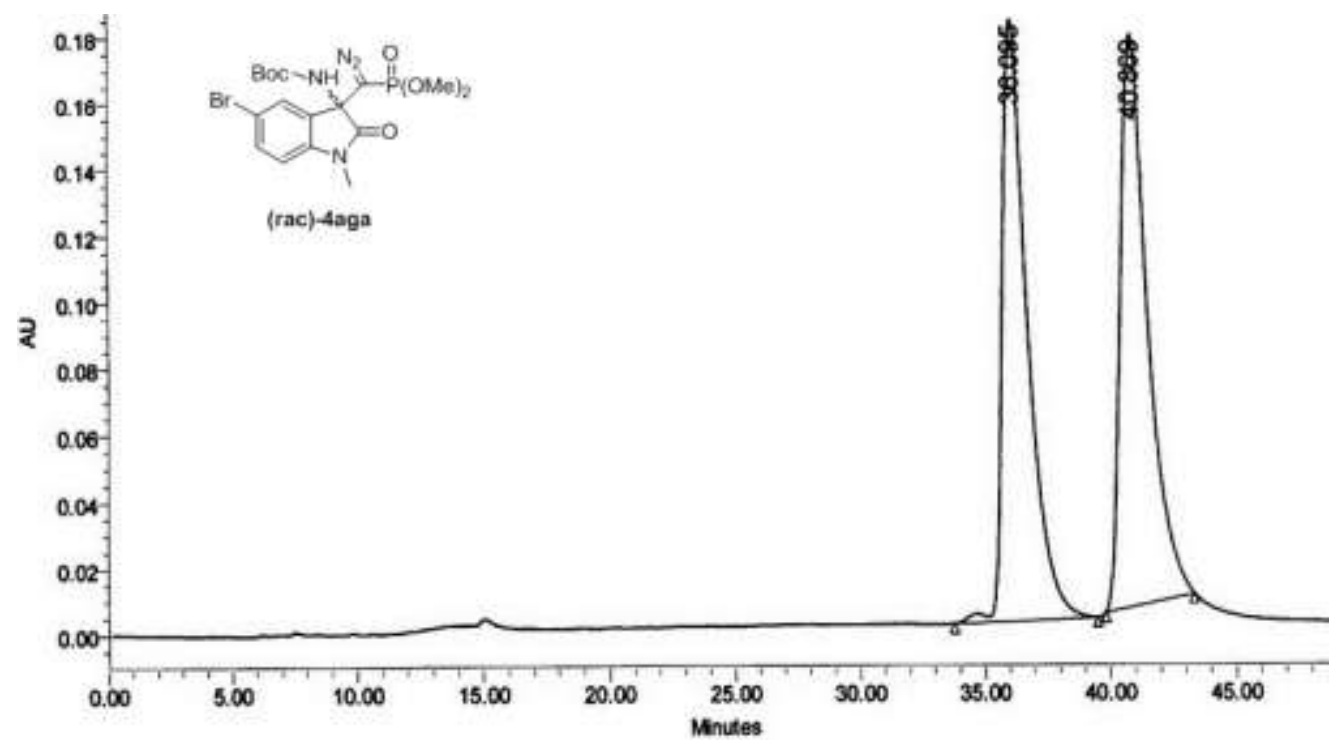

\begin{tabular}{|c|c|c|c|c|c|}
\hline & $\begin{array}{c}\text { Rा } \\
(\text { min })\end{array}$ & $\begin{array}{c}\text { Area } \\
\left(\mu N^{\prime} \text { sec }\right)\end{array}$ & \% Area & $\begin{array}{c}\text { Height } \\
(\mu \mathrm{M})\end{array}$ & $\begin{array}{c}\% \\
\text { Height }\end{array}$ \\
\hline 1 & 36.095 & 12598737 & 49.38 & 181188 & 51.26 \\
\hline 2 & 40.869 & 12914329 & 50.62 & 172307 & 48.74 \\
\hline
\end{tabular}

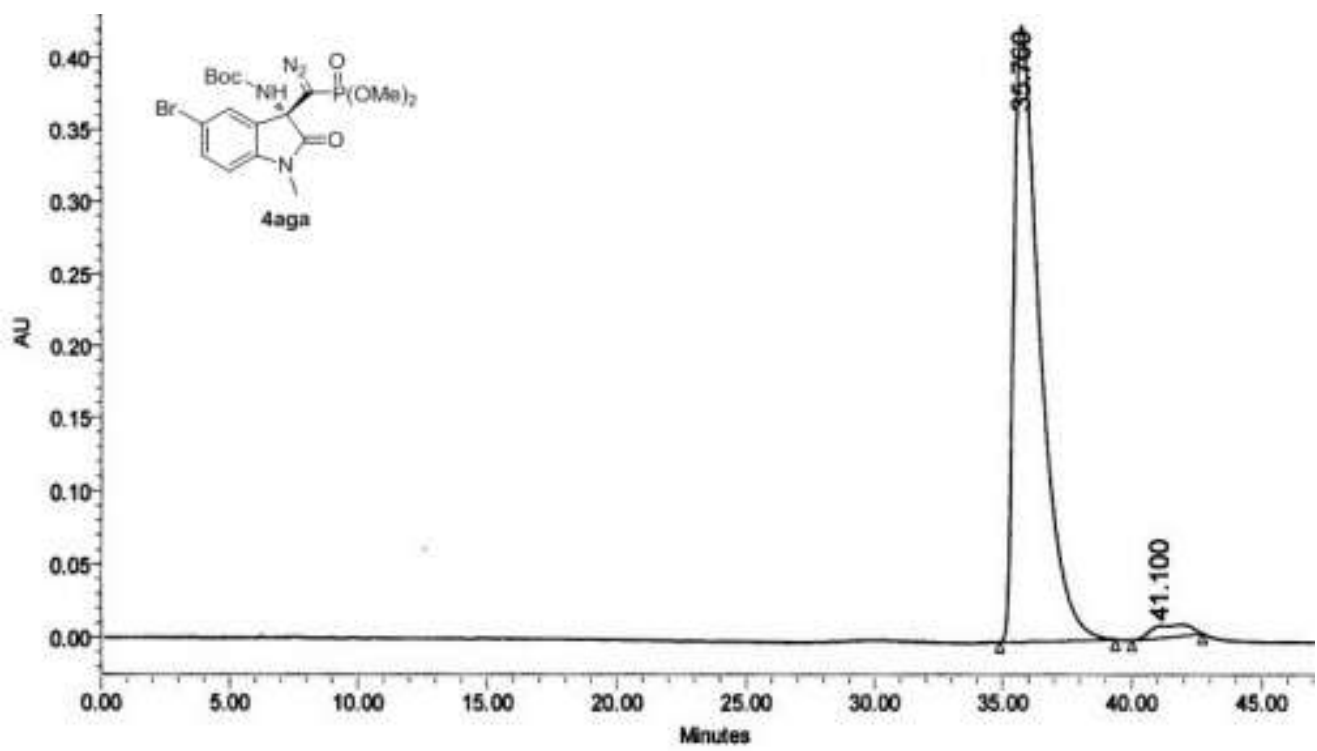

\begin{tabular}{|c|c|c|r|r|r|}
\hline & $\begin{array}{c}\text { RT } \\
\text { (min) }\end{array}$ & $\begin{array}{c}\text { Area } \\
\left(\mu V^{*} \mathrm{sec}\right)\end{array}$ & \% Area & $\begin{array}{c}\text { Height } \\
(\mu \mathrm{V})\end{array}$ & $\begin{array}{c}\% \\
\text { Height }\end{array}$ \\
\hline 1 & 35.760 & 29812353 & 97.31 & 425022 & 98.13 \\
\hline 2 & 41.100 & 824798 & 2.69 & 8081 & 1.87 \\
\hline
\end{tabular}




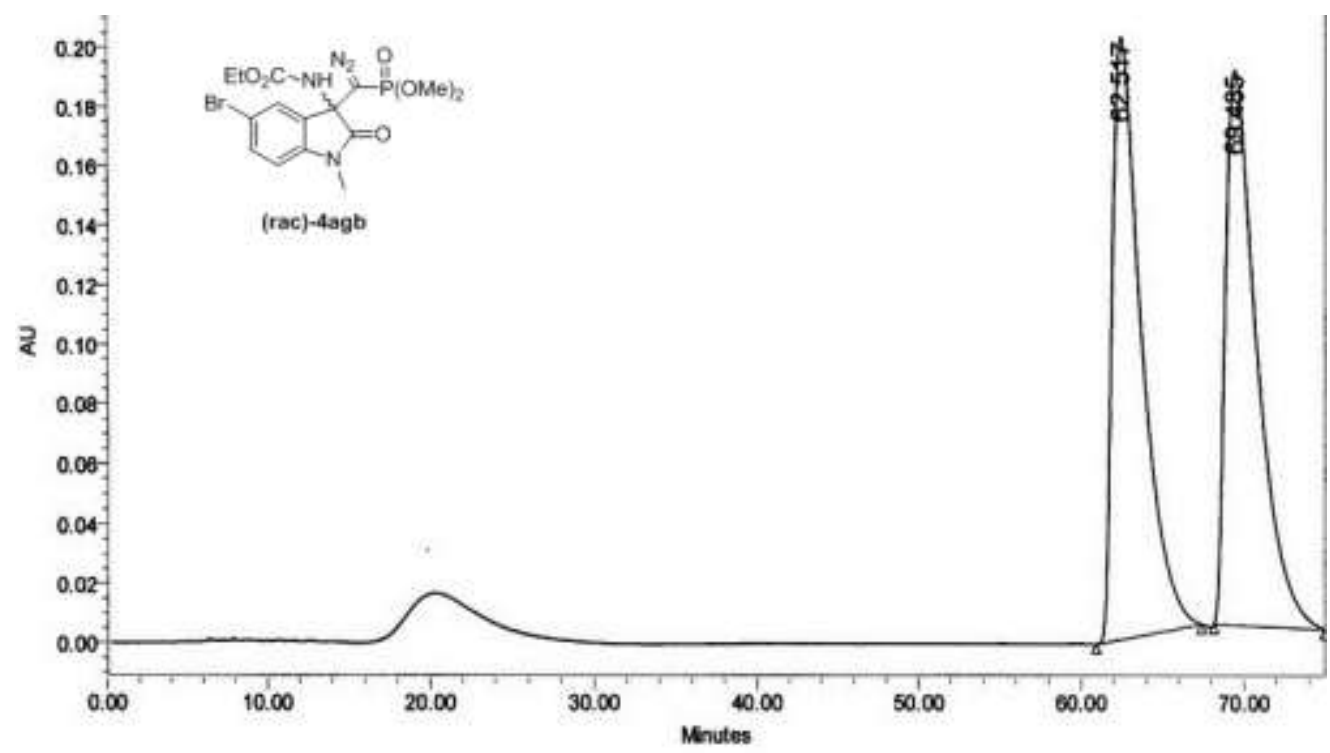

\begin{tabular}{|c|c|c|c|c|c|}
\hline & $\begin{array}{c}\text { RI } \\
(\mathrm{min})\end{array}$ & $\begin{array}{c}\text { Area } \\
(\mu \mathrm{V} \text { "sec })\end{array}$ & \% Area & $\begin{array}{c}\text { Height } \\
(\mu \mathrm{V})\end{array}$ & $\begin{array}{c}\% \\
\text { Height }\end{array}$ \\
\hline 1 & 62.517 & 25026944 & 50.28 & 201818 & 52.07 \\
\hline 2 & 69.485 & 24751633 & 49.72 & 185773 & 47.93 \\
\hline
\end{tabular}

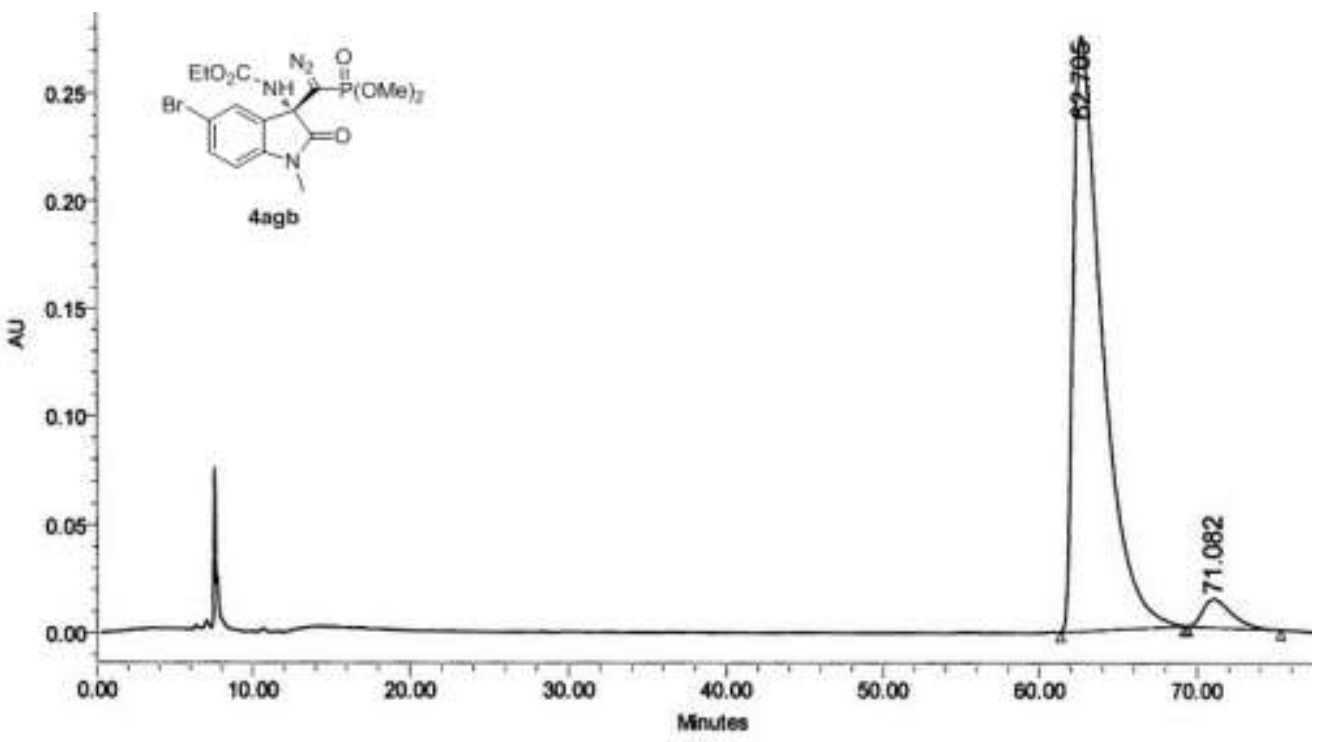

\begin{tabular}{|c|c|c|c|c|c|}
\hline & $\begin{array}{c}\text { RT } \\
(\text { min })\end{array}$ & $\begin{array}{c}\text { Area } \\
\left(\mu \mathrm{N}^{*} \mathrm{sec}\right)\end{array}$ & \% Area & $\begin{array}{c}\text { Height } \\
(\mu \mathrm{N})\end{array}$ & $\begin{array}{c}\% \\
\text { Height }\end{array}$ \\
\hline 1 & 62.705 & 35222743 & 95.53 & 275420 & 95.47 \\
\hline 2 & 71.082 & 1647245 & 4.47 & 13076 & 4.53 \\
\hline
\end{tabular}




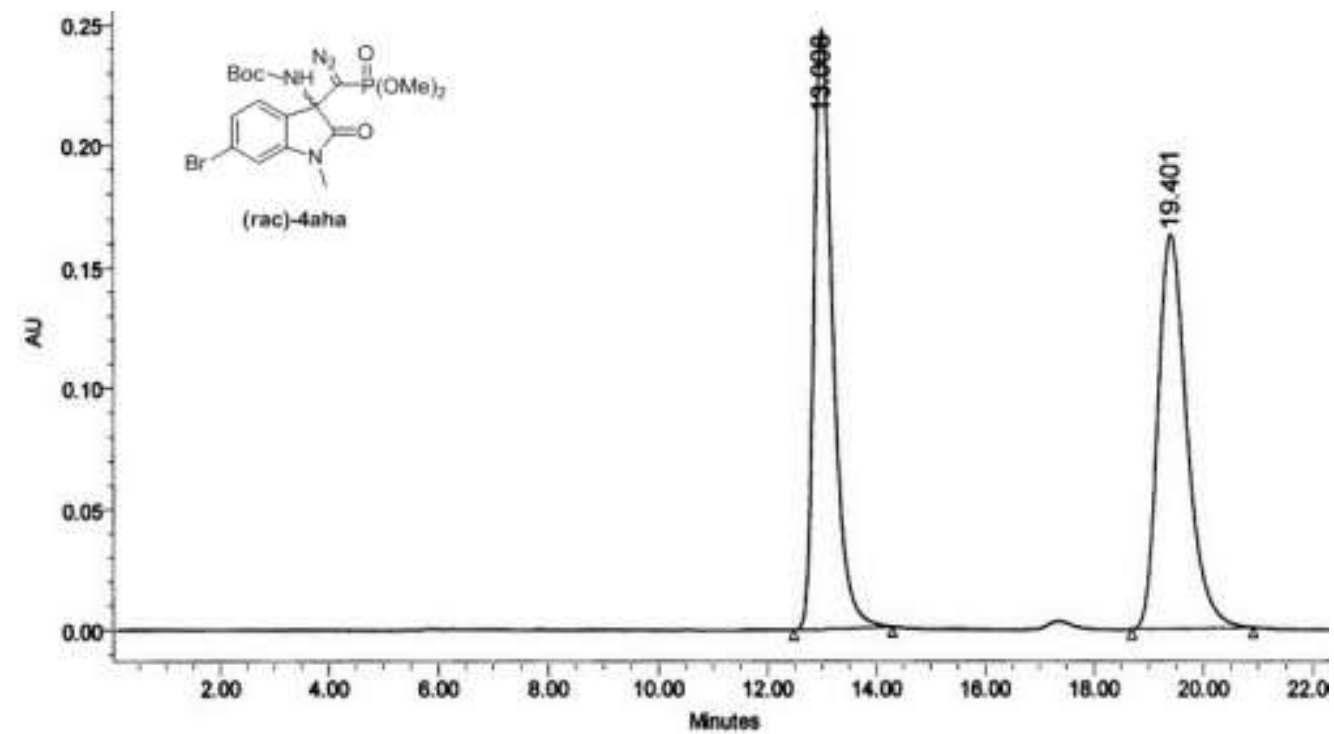

\begin{tabular}{|c|c|c|c|c|c|}
\hline & $\begin{array}{c}\text { RT } \\
(\mathrm{min})\end{array}$ & $\begin{array}{c}\text { Area } \\
(\mu \mathrm{N} / \mathrm{sec})\end{array}$ & $\%$ Area & $\begin{array}{c}\text { Height } \\
(\mu \mathrm{M})\end{array}$ & $\begin{array}{c}\% \\
\text { Height }\end{array}$ \\
\hline 1 & 13.008 & 5992035 & 50.08 & 247616 & 60.31 \\
\hline 2 & 19.401 & 5973476 & 49.92 & 162971 & 39.69 \\
\hline
\end{tabular}

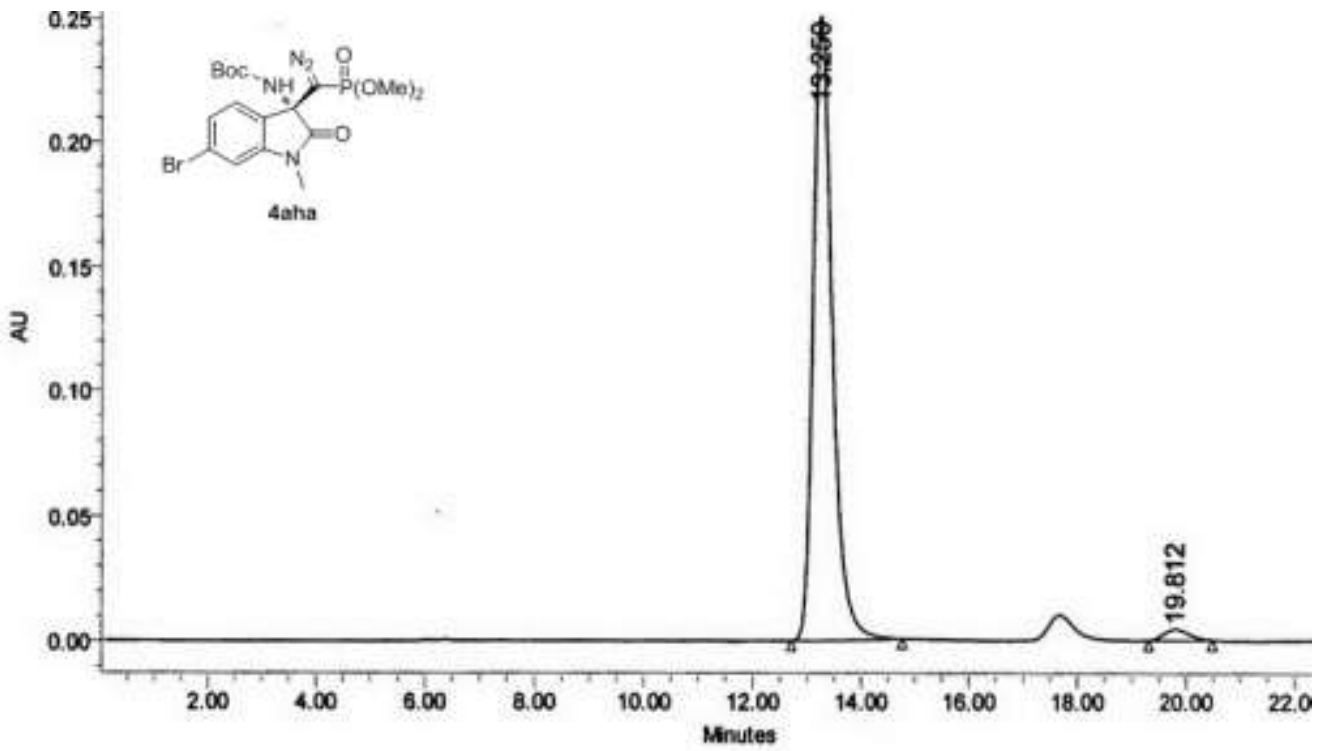

\begin{tabular}{|c|c|c|c|c|c|}
\hline & $\begin{array}{c}\text { RT } \\
(\mathrm{min})\end{array}$ & $\begin{array}{c}\text { Area } \\
(\mu \mathrm{V} \text { `sec })^{*}\end{array}$ & $\%$ Area & $\begin{array}{c}\text { Height } \\
(\mu \mathrm{V})\end{array}$ & $\begin{array}{c}\% \\
\text { Height }\end{array}$ \\
\hline 1 & 13.259 & 6157413 & 97.71 & 251355 & 98.24 \\
\hline 2 & 19.812 & 144311 & 2.29 & 4499 & 1.76 \\
\hline
\end{tabular}




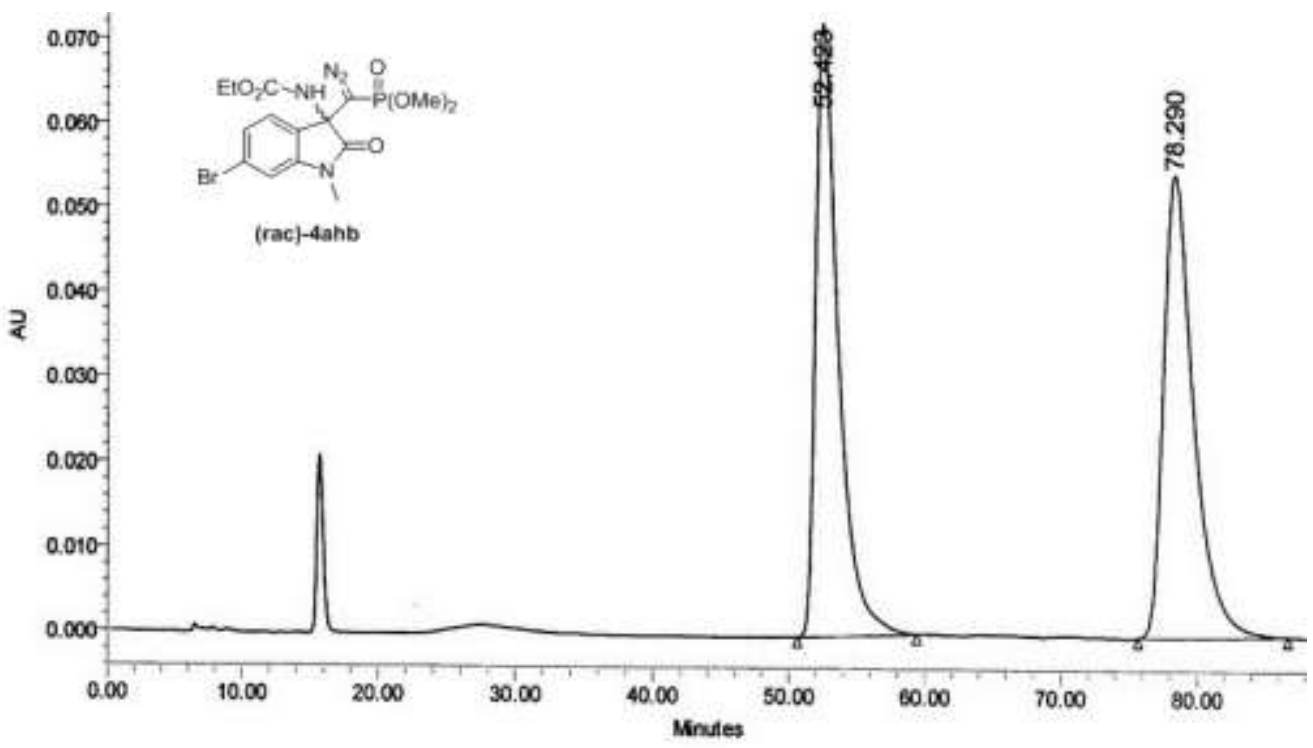

\begin{tabular}{|c|c|c|c|c|c|}
\hline & $\begin{array}{c}\text { Rा } \\
(m i n)\end{array}$ & $\begin{array}{c}\text { Area } \\
\left(\mu N^{\prime} \text { 'sec }\right)\end{array}$ & $\%$ Area & $\begin{array}{c}\text { Height } \\
(\mu \mathrm{N})\end{array}$ & $\begin{array}{c}\% \\
\text { Height }\end{array}$ \\
\hline 1 & 52.423 & 8422272 & 50.16 & 72327 & 57.03 \\
\hline 2 & 78.290 & 8367150 & 49.84 & 54493 & 42.97 \\
\hline
\end{tabular}

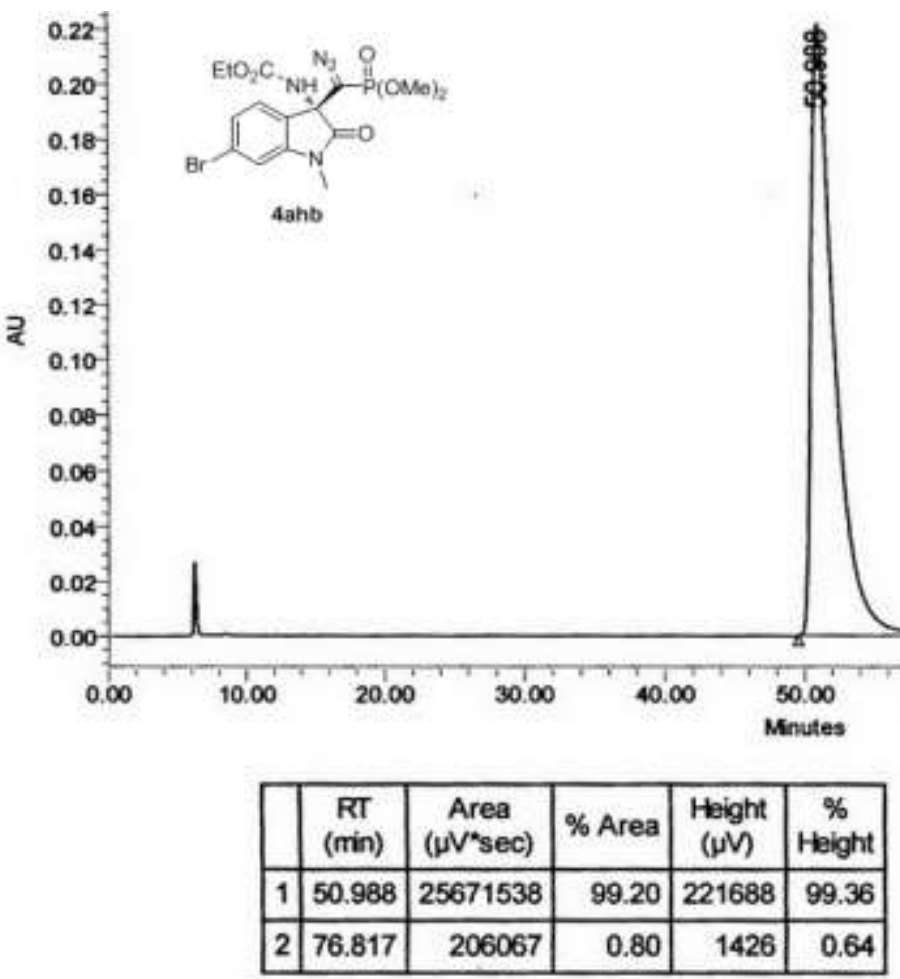




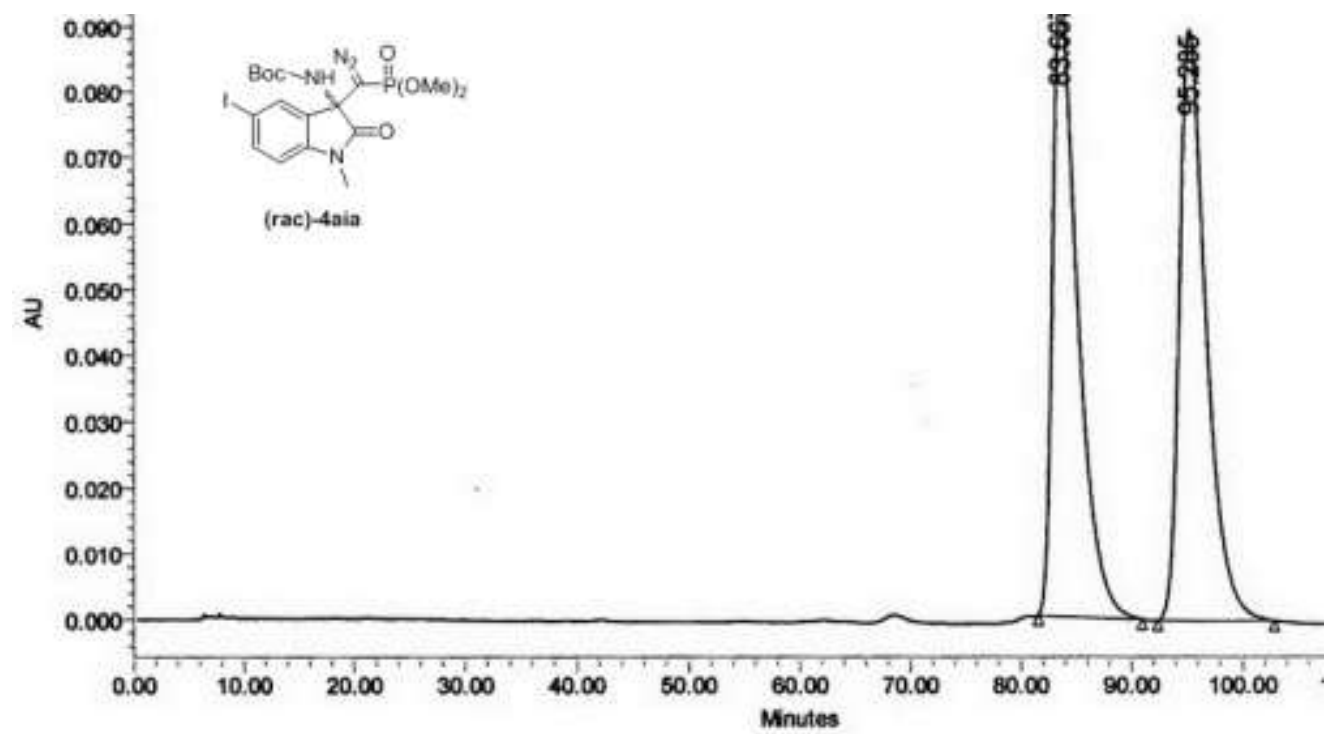

\begin{tabular}{|c|c|c|c|c|c|}
\hline & $\begin{array}{c}\text { RT } \\
(\mathrm{min})\end{array}$ & $\begin{array}{c}\text { Area } \\
(\mu \mathrm{N} \text { *sec })\end{array}$ & \% Area & $\begin{array}{c}\text { Height } \\
(\mu \mathrm{N})\end{array}$ & $\begin{array}{c}\% \\
\text { Height }\end{array}$ \\
\hline 1 & 83.667 & 14810873 & 49.77 & 93553 & 51.04 \\
\hline 2 & 95.285 & 14948308 & 50.23 & 89741 & 48.96 \\
\hline
\end{tabular}

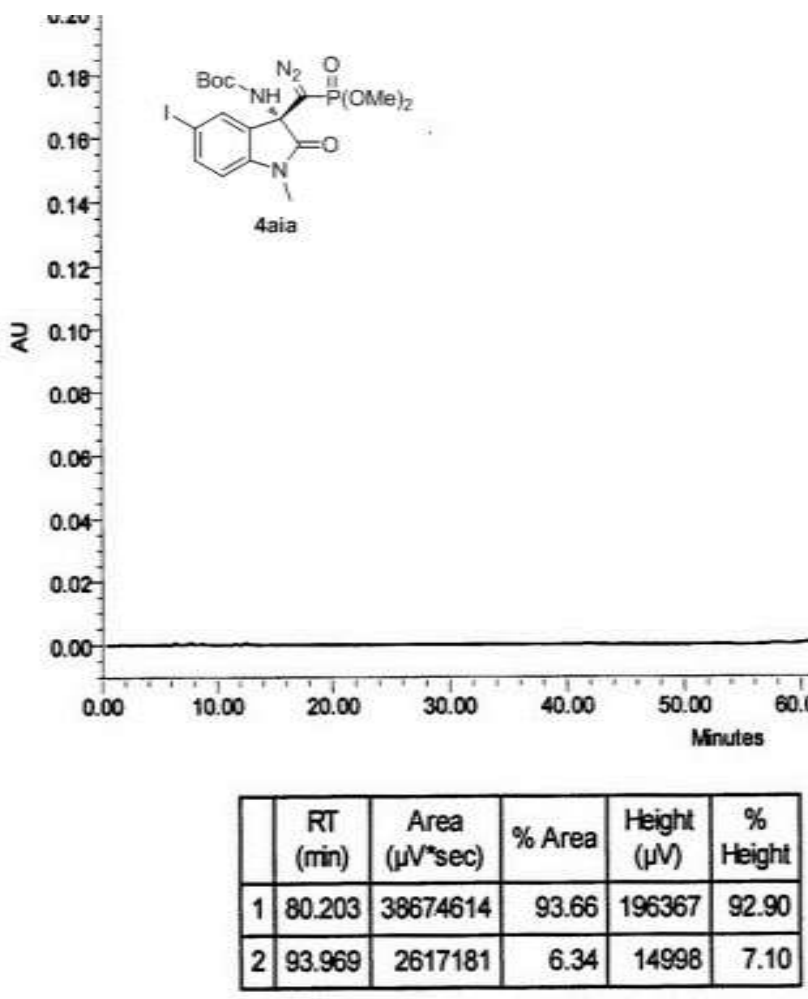




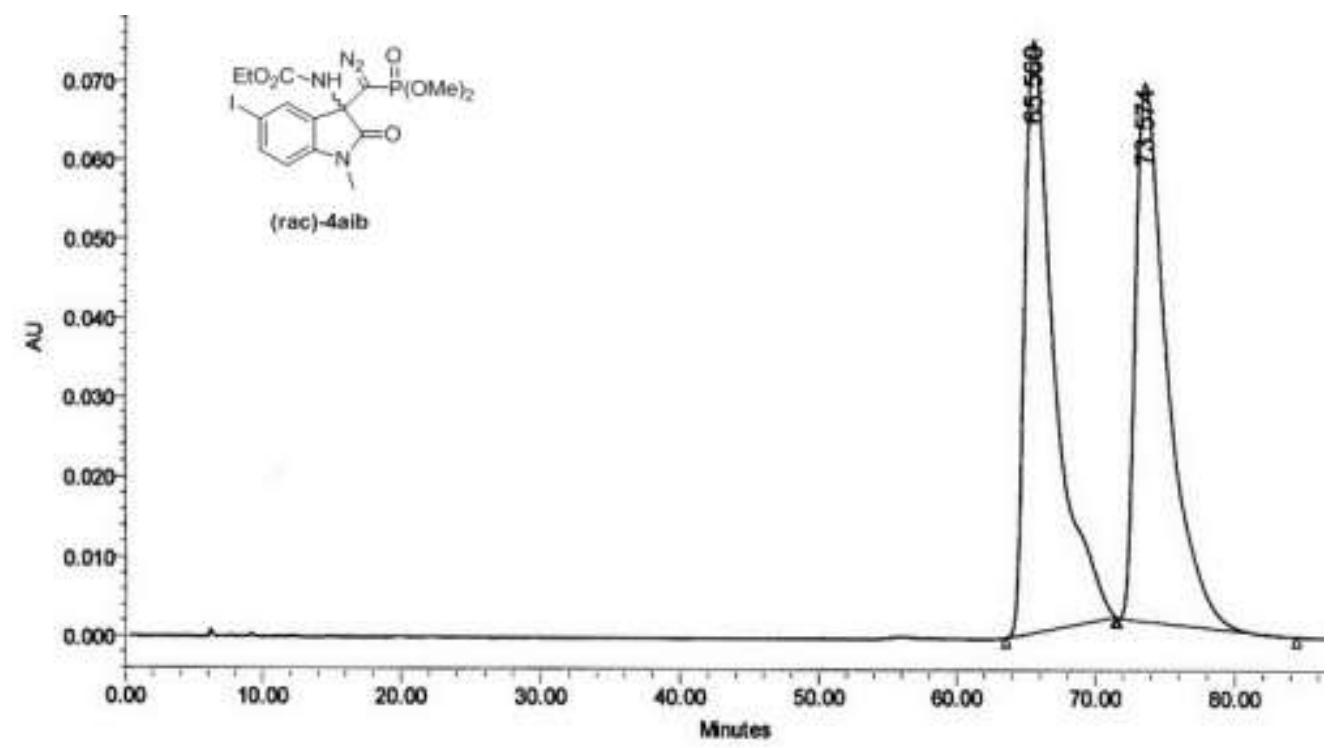

\begin{tabular}{|c|c|c|c|c|c|}
\hline & $\begin{array}{c}\text { RT } \\
(\mathrm{min})\end{array}$ & $\begin{array}{c}\text { Area } \\
\left(\mu \mathrm{N}^{*} \mathrm{sec}\right)\end{array}$ & $\%$ Area & $\begin{array}{c}\text { Height } \\
(\mu \mathrm{M})\end{array}$ & $\begin{array}{c}\% \\
\text { Height }\end{array}$ \\
\hline 1 & 65.560 & 11127901 & 50.98 & 74620 & 52.42 \\
\hline 2 & 73.574 & 10699868 & 49.02 & 67742 & 47.58 \\
\hline
\end{tabular}

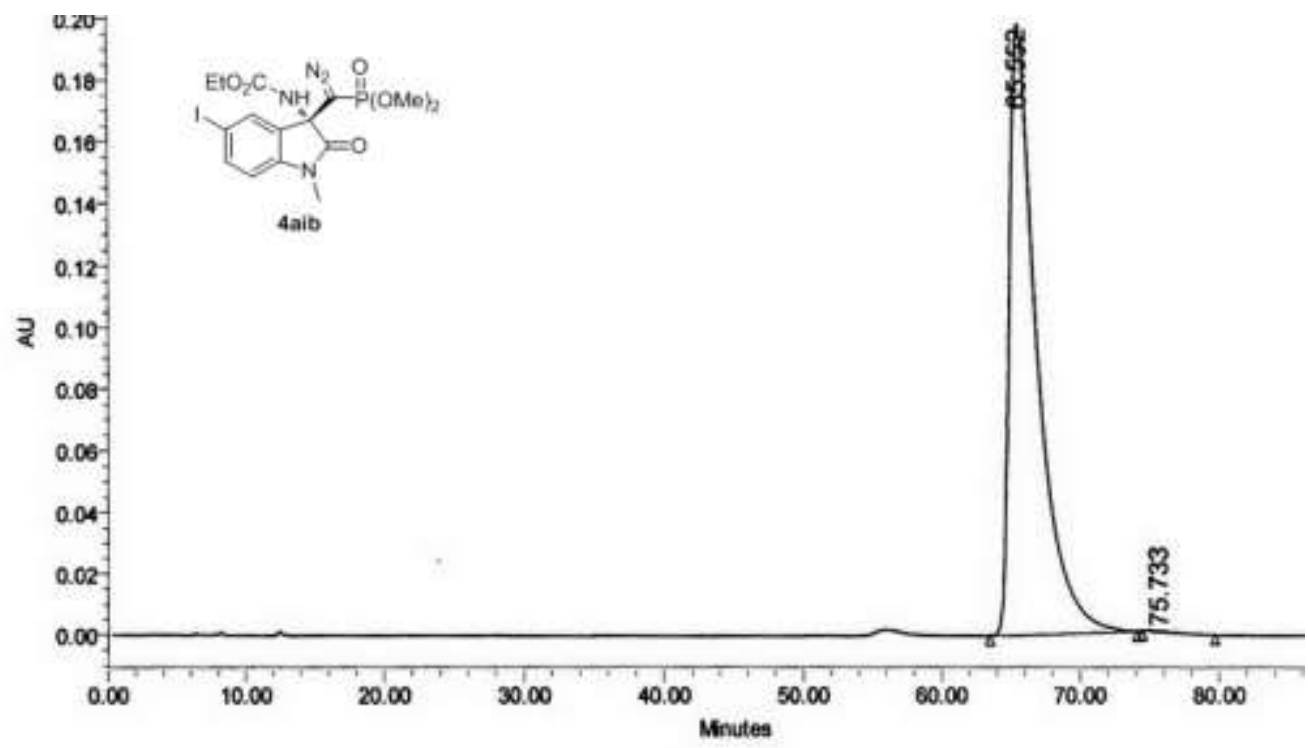

\begin{tabular}{|c|c|r|r|r|r|}
\hline & $\begin{array}{c}\text { RI } \\
(\mathrm{min})\end{array}$ & $\begin{array}{c}\text { Area } \\
(\mu \mathrm{V} \text { 'sec })\end{array}$ & \% Area & $\begin{array}{c}\text { Height } \\
(\mu \mathrm{V})\end{array}$ & $\begin{array}{c}\% \\
\text { Height }\end{array}$ \\
\hline 1 & 65.552 & 27475867 & 99.85 & 198434 & 99.83 \\
\hline 2 & 75.733 & 41446 & 0.15 & 346 & 0.17 \\
\hline
\end{tabular}




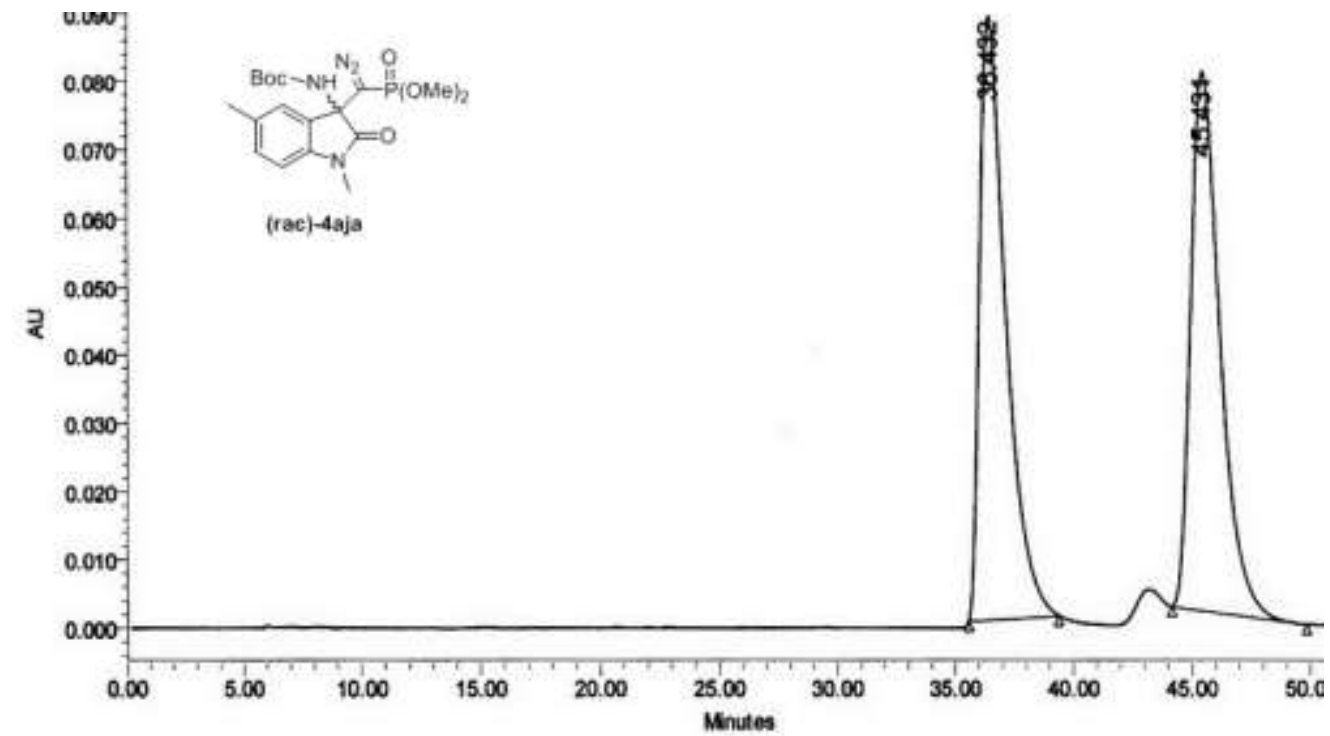

\begin{tabular}{|c|c|c|c|c|c|}
\hline & $\begin{array}{c}\text { Rा } \\
(\mathrm{min})\end{array}$ & $\begin{array}{c}\text { Area } \\
\left(\mu \mathrm{V}^{\prime} \mathrm{sec}\right)\end{array}$ & \% Area & $\begin{array}{c}\text { Height } \\
(\mu \mathrm{M})\end{array}$ & $\begin{array}{c}\% \\
\text { Height }\end{array}$ \\
\hline 1 & 36.432 & 6854780 & 50.30 & 88492 & 52.87 \\
\hline 2 & 45.431 & 6772720 & 49.70 & 78871 & 47.13 \\
\hline
\end{tabular}

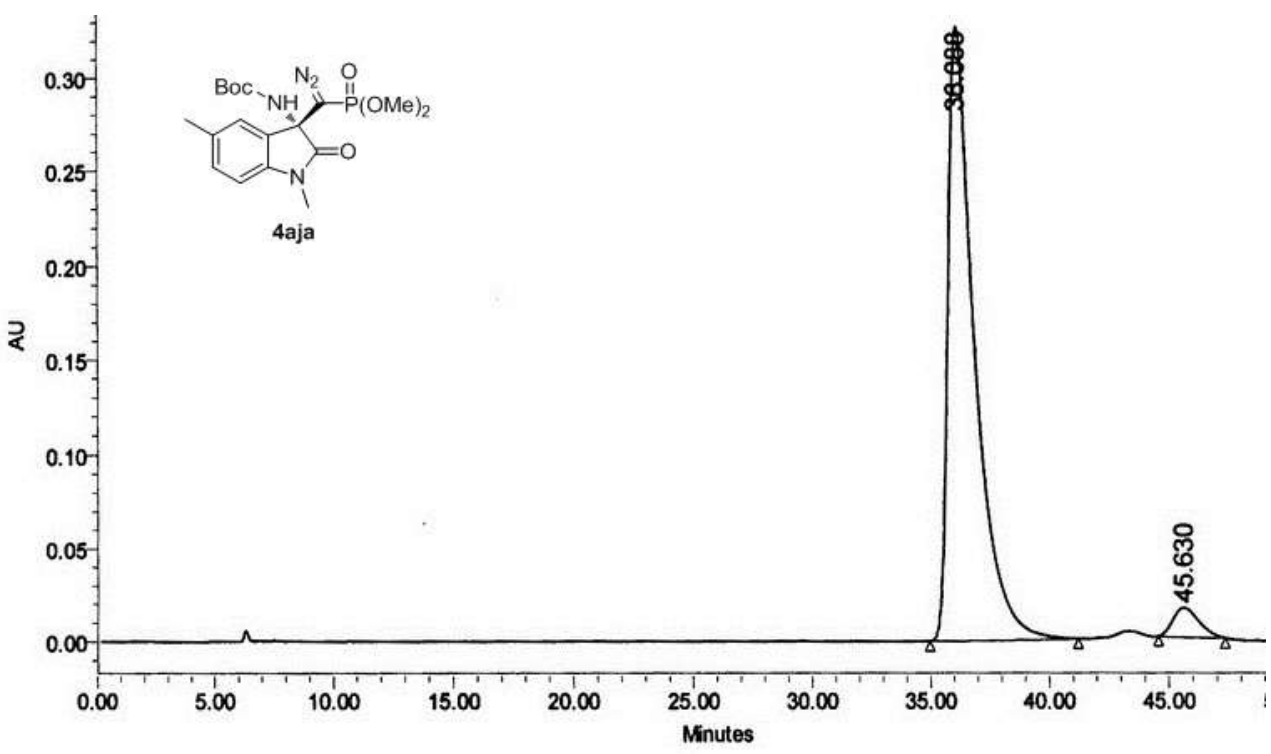

\begin{tabular}{|c|c|c|c|c|c|}
\hline & $\begin{array}{c}\text { RT } \\
(\mathrm{min})\end{array}$ & $\begin{array}{c}\text { Area } \\
\left(\mu \mathrm{V}^{*} \mathrm{sec}\right)\end{array}$ & $\%$ Area & $\begin{array}{c}\text { Height } \\
(\mu \mathrm{V})\end{array}$ & $\begin{array}{c}\% \\
\text { Height }\end{array}$ \\
\hline 1 & 36.088 & 25513966 & 95.64 & 327609 & 95.46 \\
\hline 2 & 45.630 & 1162919 & 4.36 & 15586 & 4.54 \\
\hline
\end{tabular}




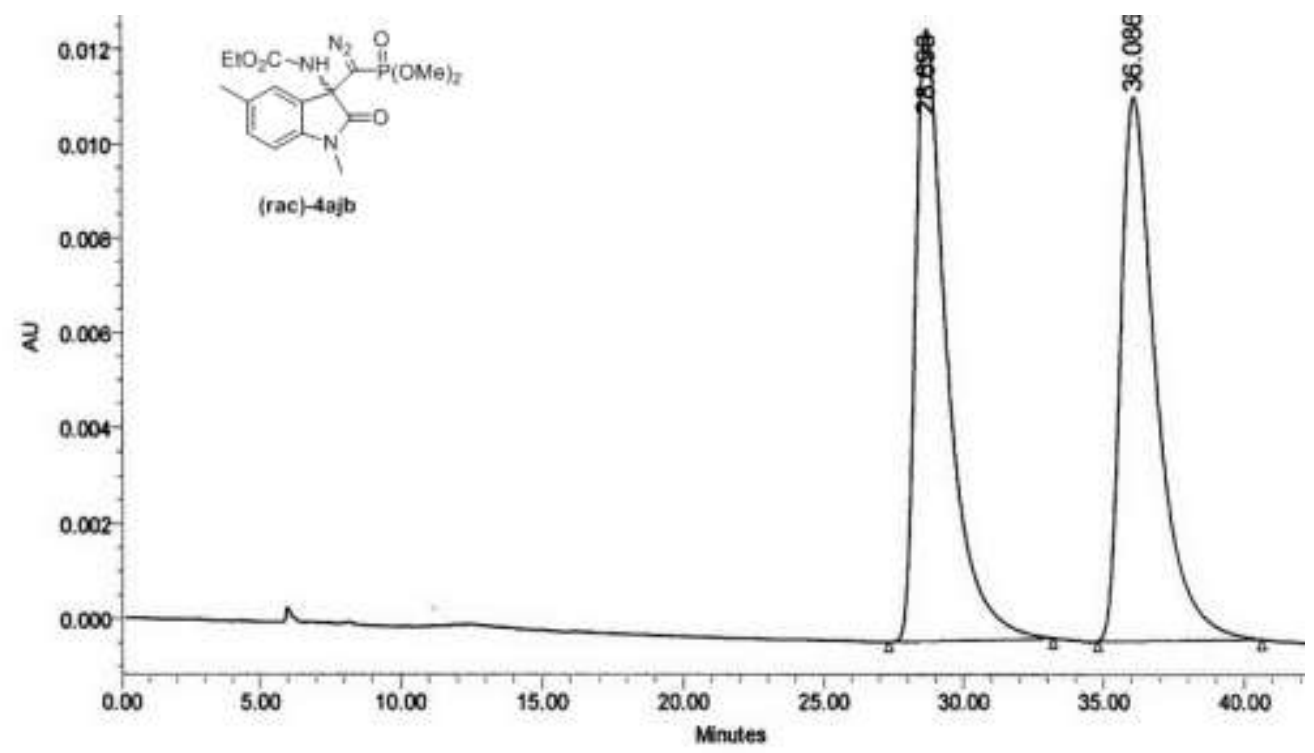

\begin{tabular}{|c|c|c|c|c|c|}
\hline & $\begin{array}{c}\text { RT } \\
(\mathrm{min})\end{array}$ & $\begin{array}{c}\text { Area } \\
\left(\mu N^{*} \mathrm{sec}\right)\end{array}$ & $\%$ Area & $\begin{array}{c}\text { Height } \\
(\mu \mathrm{M})\end{array}$ & $\begin{array}{c}\% \\
\text { Height }\end{array}$ \\
\hline 1 & 28.698 & 1013202 & 50.27 & 12863 & 52.97 \\
\hline 2 & 36.086 & 1002259 & 49.73 & 11420 & 47.03 \\
\hline
\end{tabular}

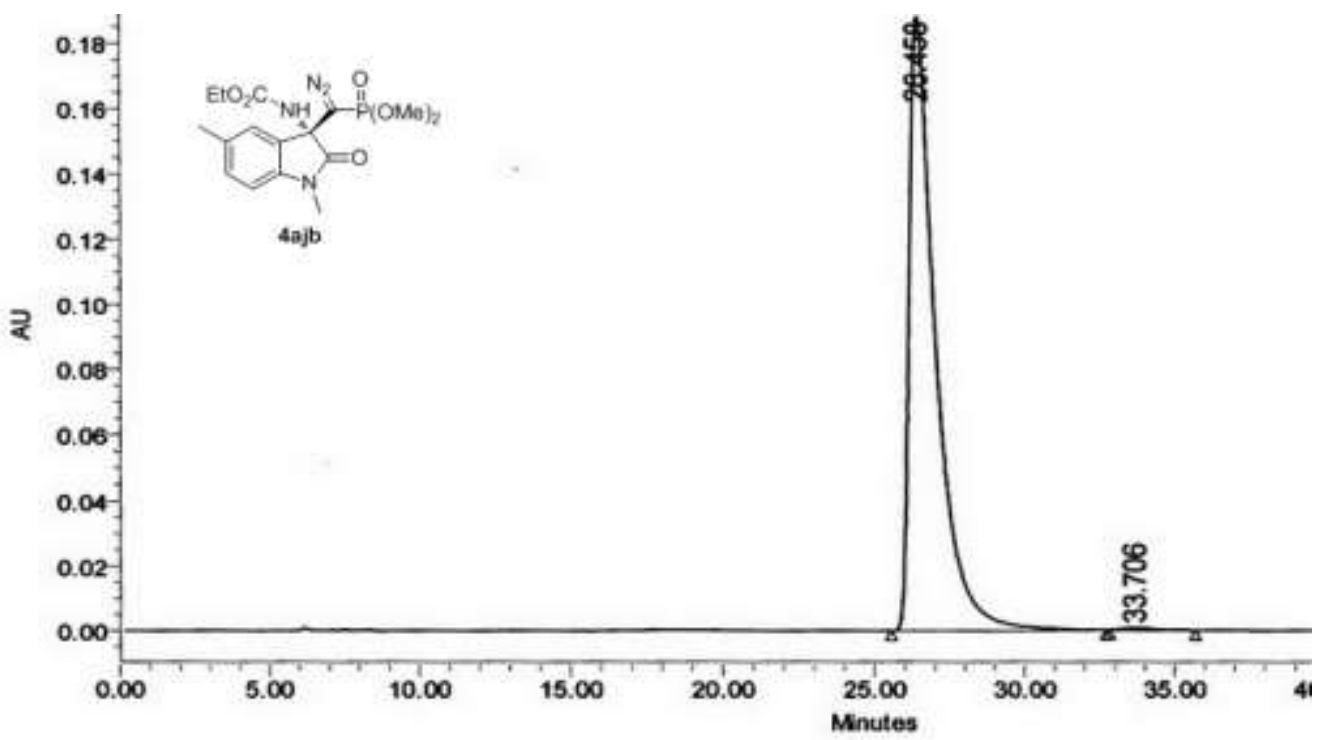

\begin{tabular}{|c|c|r|r|r|r|}
\hline & $\begin{array}{c}\text { RT } \\
(\min )\end{array}$ & $\begin{array}{c}\text { Area } \\
\left(\mu \mathrm{N}^{*} \text { sec }\right)\end{array}$ & \% Area & $\begin{array}{c}\text { Height } \\
(\mu \mathrm{N})\end{array}$ & $\begin{array}{c}\% \\
\text { Height }\end{array}$ \\
\hline 1 & 26.458 & 11429053 & 99.60 & 188031 & 99.65 \\
\hline 2 & 33.706 & 46309 & 0.40 & 655 & 0.35 \\
\hline
\end{tabular}




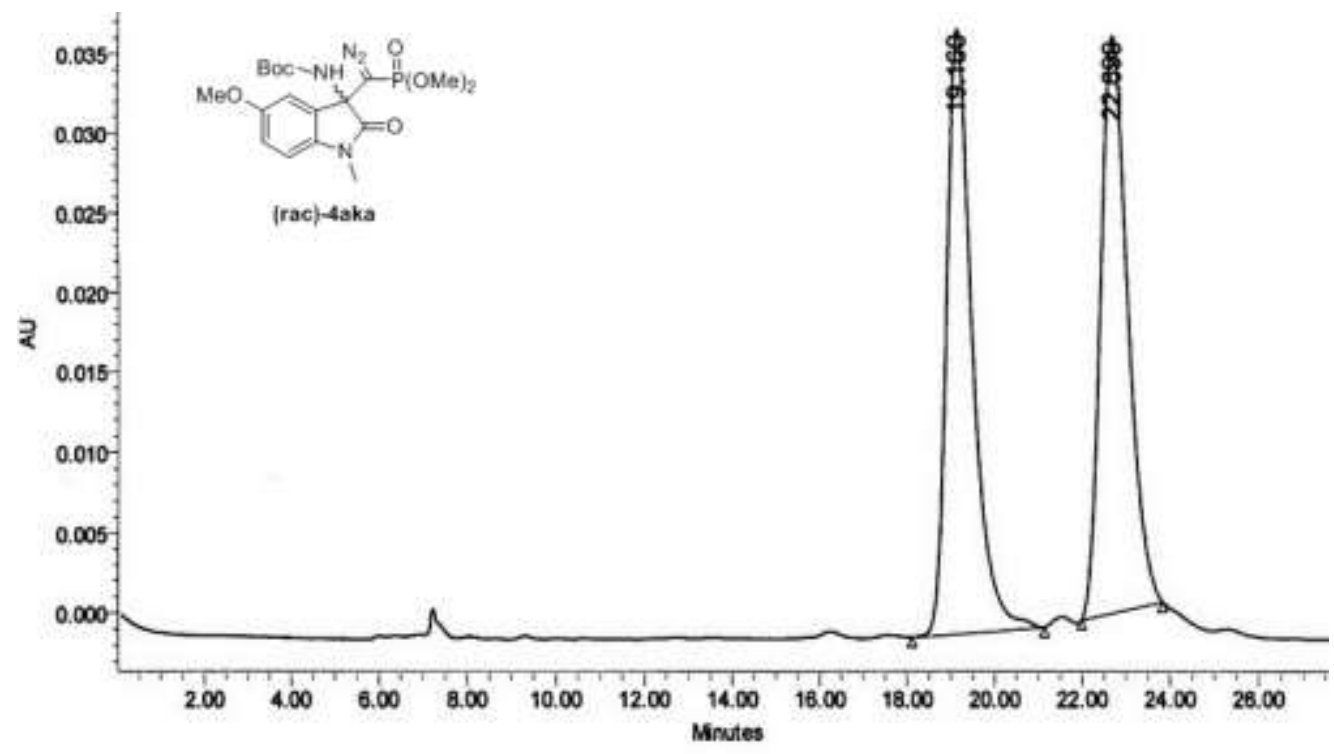

\begin{tabular}{|c|c|c|c|c|c|}
\hline & $\begin{array}{c}\text { RT } \\
(\mathrm{min})\end{array}$ & $\begin{array}{c}\text { Area } \\
(\mu \mathrm{N} \text { "sec })\end{array}$ & \% Area & $\begin{array}{c}\text { Height } \\
(\mu \mathrm{V})\end{array}$ & $\begin{array}{c}\% \\
\text { Height }\end{array}$ \\
\hline 1 & 19.160 & 1535975 & 49.98 & 37850 & 51.15 \\
\hline 2 & 22.699 & 1537246 & 50.02 & 36148 & 48.85 \\
\hline
\end{tabular}

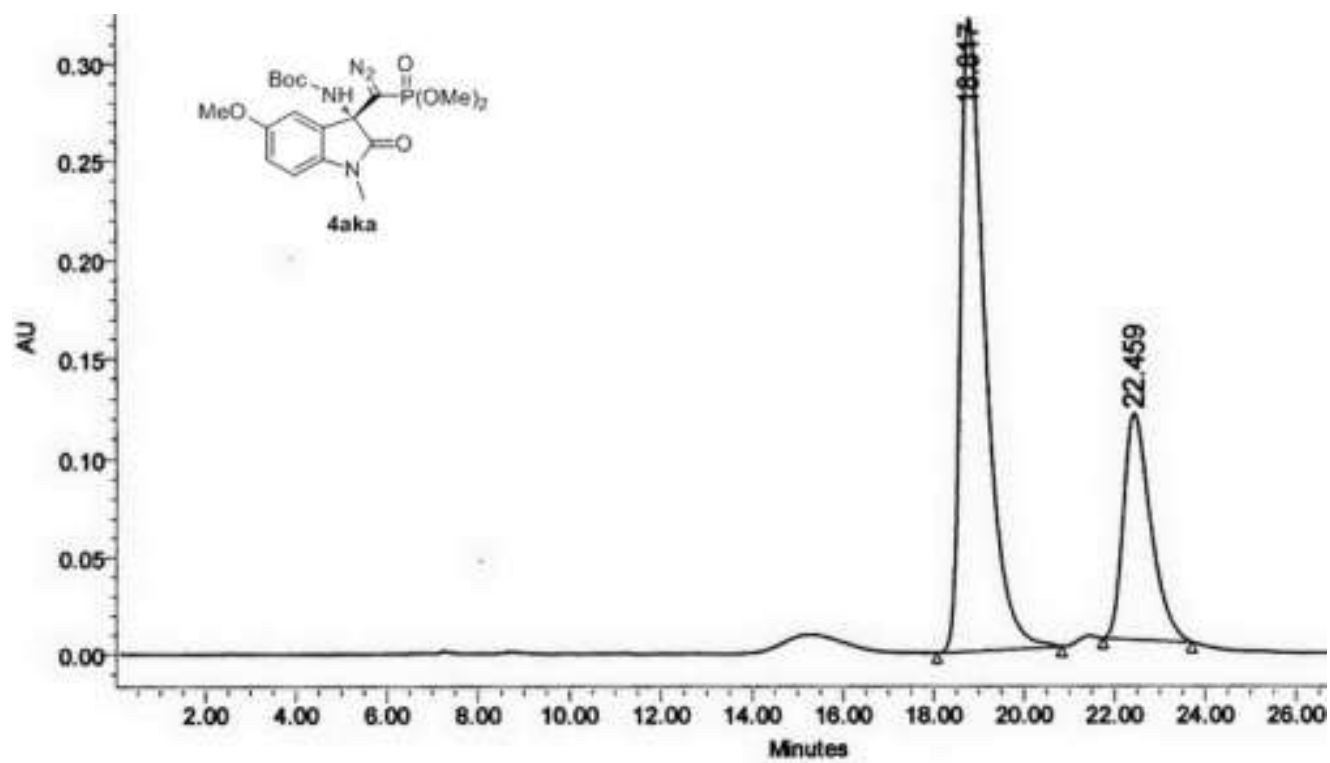

\begin{tabular}{|c|c|c|c|c|c|}
\hline & $\begin{array}{c}\text { RT } \\
(m i n)\end{array}$ & $\begin{array}{c}\text { Area } \\
\left(\mu N^{*} \text { sec }\right)\end{array}$ & \% Area & $\begin{array}{c}\text { Height } \\
(\mu \mathrm{V})\end{array}$ & $\begin{array}{c}\% \\
\text { Height }\end{array}$ \\
\hline 1 & 18.817 & 12296195 & 71.71 & 321792 & 73.68 \\
\hline 2 & 22.459 & 4851959 & 28.29 & 114937 & 26.32 \\
\hline
\end{tabular}




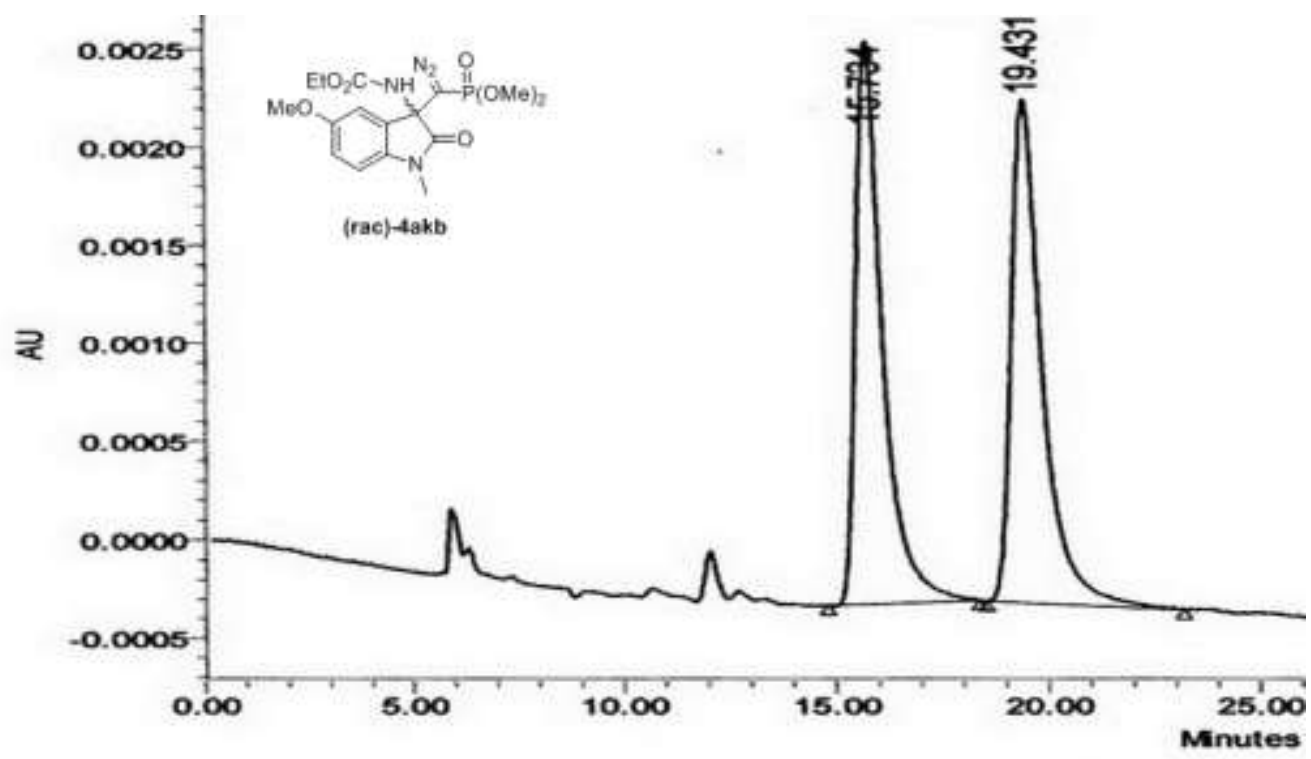

\begin{tabular}{|c|c|r|r|r|r|}
\hline & $\begin{array}{c}\text { RT } \\
(\mathrm{min})\end{array}$ & $\begin{array}{c}\text { Area } \\
\left(\mu N^{*} \text { sec }\right)\end{array}$ & $\%$ Area & $\begin{array}{c}\text { Height } \\
\left.(\mu)^{\prime}\right)\end{array}$ & $\begin{array}{c}\% \\
\text { Height }\end{array}$ \\
\hline 1 & 15.734 & 125555 & 49.48 & 2866 & 52.81 \\
\hline 2 & 19.431 & 128172 & 50.52 & 2561 & 47.19 \\
\hline
\end{tabular}

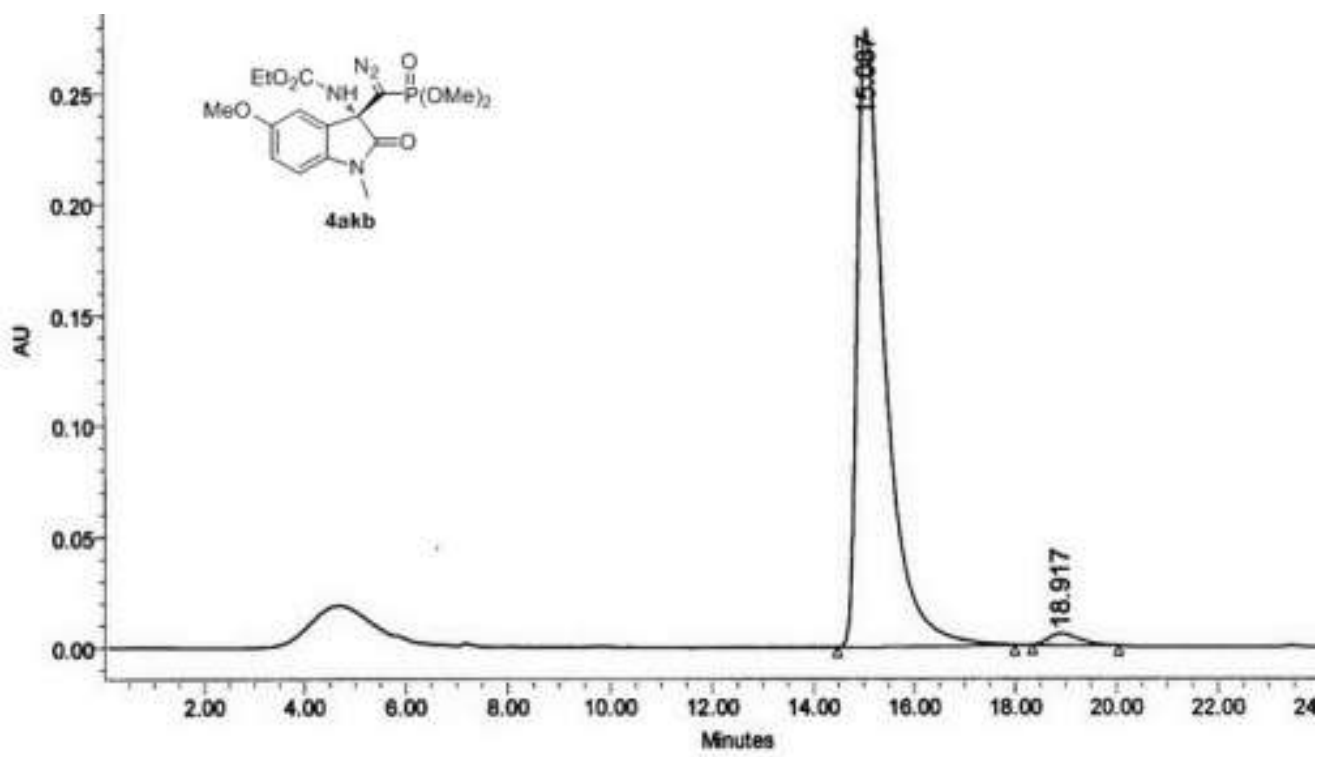

\begin{tabular}{|c|c|r|r|r|r|}
\hline & $\begin{array}{c}\text { RT } \\
(\mathrm{min})\end{array}$ & $\begin{array}{c}\text { Area } \\
(\mu \mathrm{V} \text { "sec })\end{array}$ & \% Area & $\begin{array}{c}\text { Height } \\
(\mu \mathrm{V})\end{array}$ & $\begin{array}{c}\% \\
\text { Height }\end{array}$ \\
\hline 1 & 15.087 & 10686513 & 97.91 & 278176 & 98.15 \\
\hline 2 & 18.917 & 227795 & 2.09 & 5246 & 1.85 \\
\hline
\end{tabular}



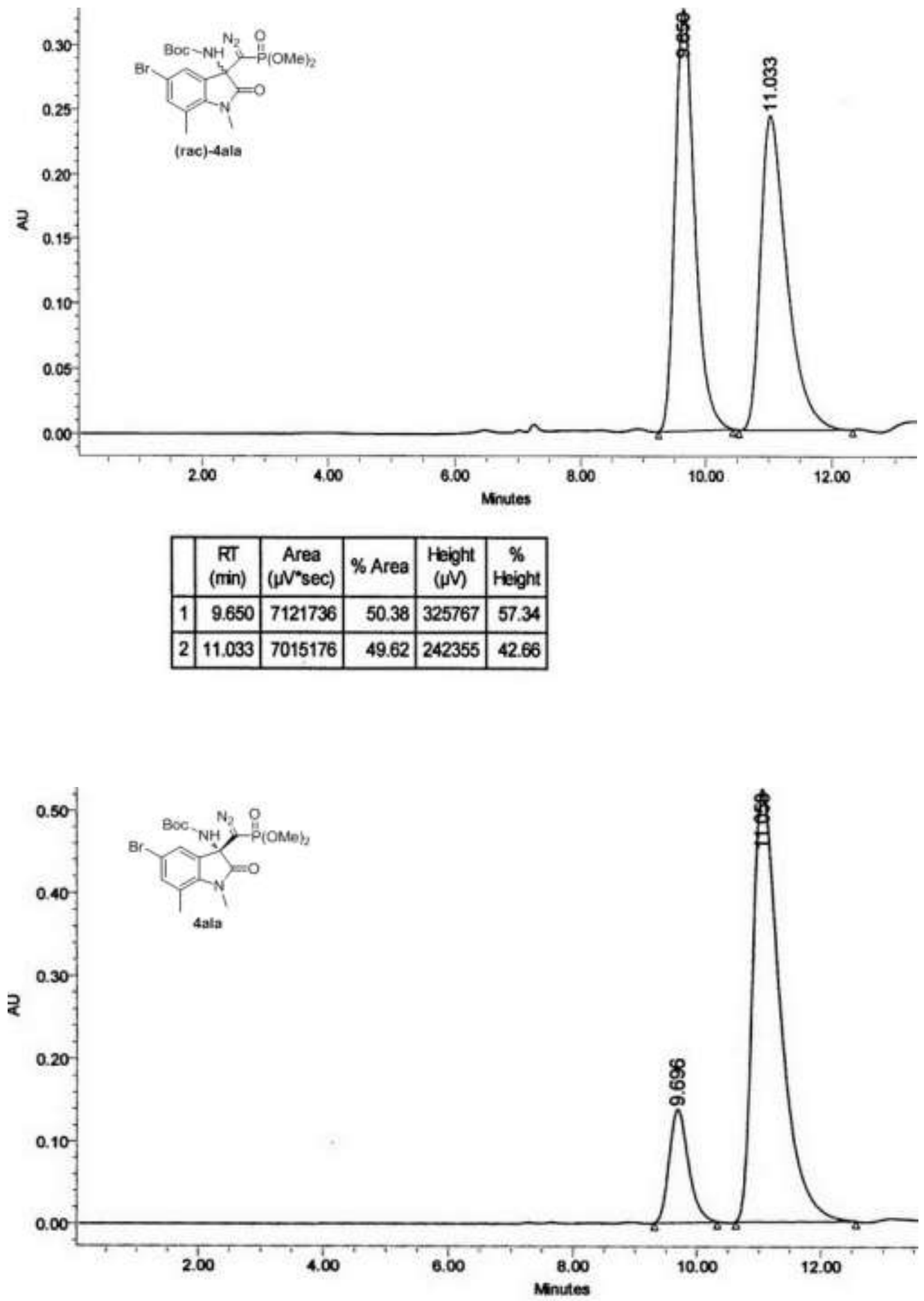

\begin{tabular}{|c|c|c|c|c|c|}
\hline & $\begin{array}{c}\text { RT } \\
(\mathrm{min})\end{array}$ & $\begin{array}{c}\text { Area } \\
(\mu \mathrm{V} \text { *sec })\end{array}$ & $\%$ Area & $\begin{array}{c}\text { Height } \\
(\mu \mathrm{V})\end{array}$ & $\begin{array}{c}\% \\
\text { Height }\end{array}$ \\
\hline 1 & 9.696 & 2996628 & 15.55 & 138507 & 20.87 \\
\hline 2 & 11.058 & 16276619 & 84.45 & 525112 & 79.13 \\
\hline
\end{tabular}




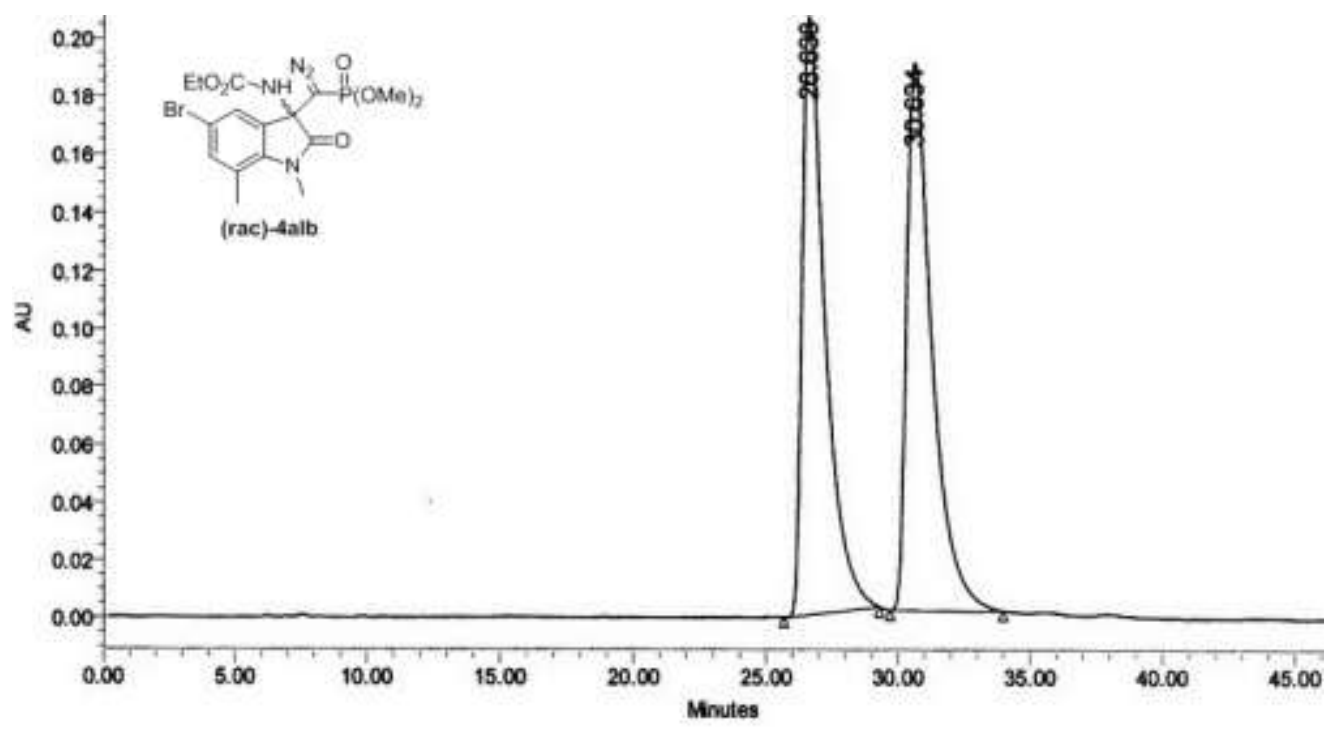

\begin{tabular}{|c|c|c|c|c|c|}
\hline & $\begin{array}{c}\text { RT } \\
(\mathrm{min})\end{array}$ & $\begin{array}{c}\text { Area } \\
\left(\mu \mathrm{N}^{2} \text { "sec }\right)\end{array}$ & \% Area & $\begin{array}{c}\text { Height } \\
(\mu \mathrm{M})\end{array}$ & $\begin{array}{c}\% \\
\text { Height }\end{array}$ \\
\hline 1 & 26.638 & 12476698 & 49.72 & 206857 & 52.24 \\
\hline 2 & 30.634 & 12618145 & 50.28 & 189132 & 47.76 \\
\hline
\end{tabular}

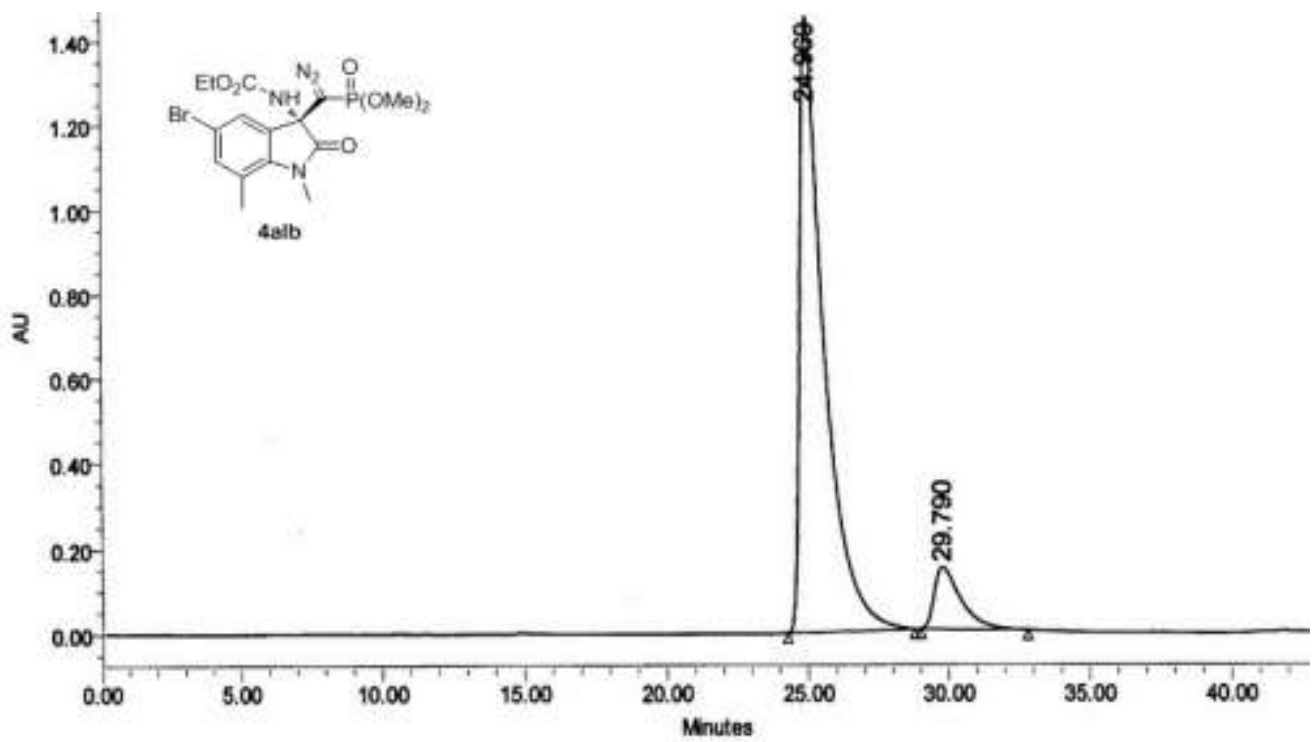

\begin{tabular}{|c|c|c|c|c|c|}
\hline & $\begin{array}{c}\text { RT } \\
(\mathrm{min})\end{array}$ & $\begin{array}{c}\text { Area } \\
(\mu \mathrm{N} \text { *sec })\end{array}$ & \% Area & $\begin{array}{c}\text { Height } \\
(\mu \mathrm{M})\end{array}$ & $\begin{array}{c}\% \\
\text { Height }\end{array}$ \\
\hline 1 & 24.969 & 86288275 & 90.06 & 1451099 & 90.86 \\
\hline 2 & 29.790 & 9522905 & 9.94 & 145952 & 9.14 \\
\hline
\end{tabular}




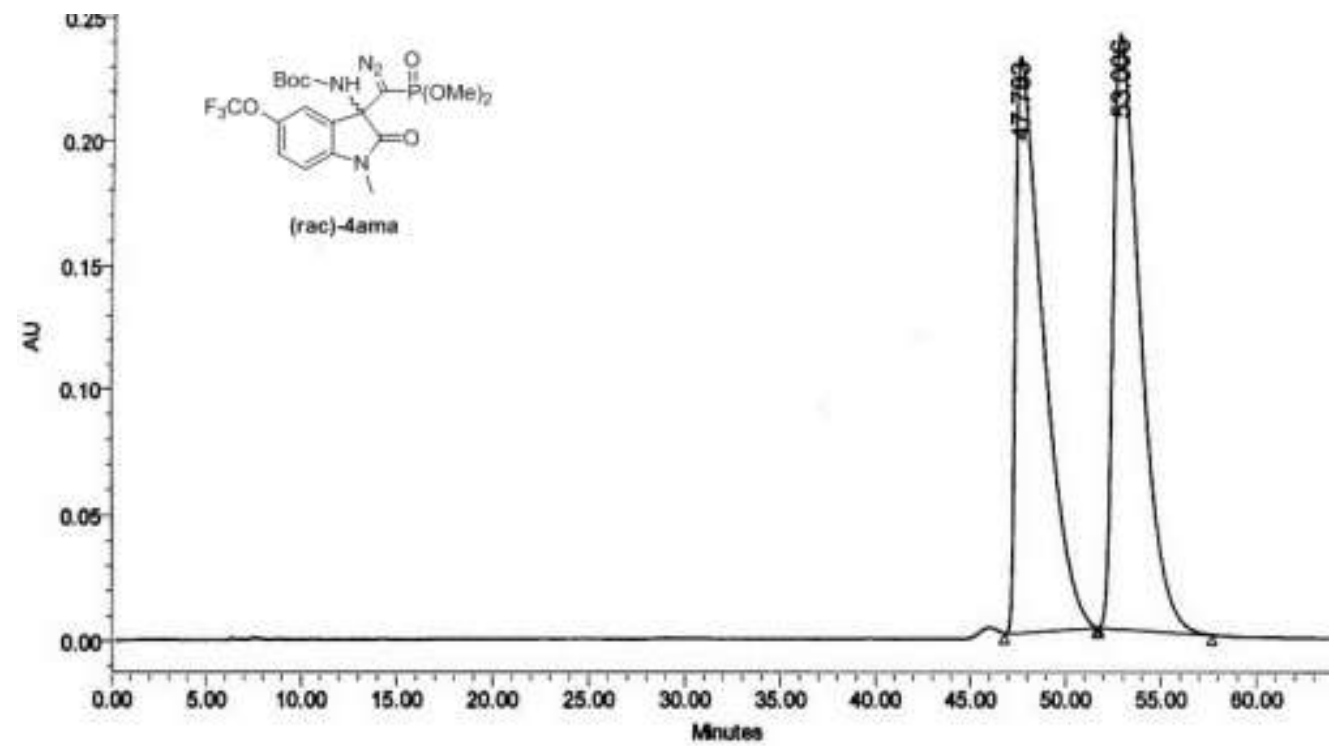

\begin{tabular}{|c|c|c|c|c|c|}
\hline & $\begin{array}{c}\text { Rा } \\
(\min )\end{array}$ & $\begin{array}{c}\text { Area } \\
\left(\mu \mathrm{N}^{*} \text { sec }\right)\end{array}$ & \% Area & $\begin{array}{c}\text { Height } \\
(\mu \mathrm{V})\end{array}$ & $\begin{array}{c}\% \\
\text { Height }\end{array}$ \\
\hline 1 & 47.783 & 23150604 & 49.65 & 230805 & 49.19 \\
\hline 2 & 53.006 & 23481581 & 50.35 & 238418 & 50.81 \\
\hline
\end{tabular}

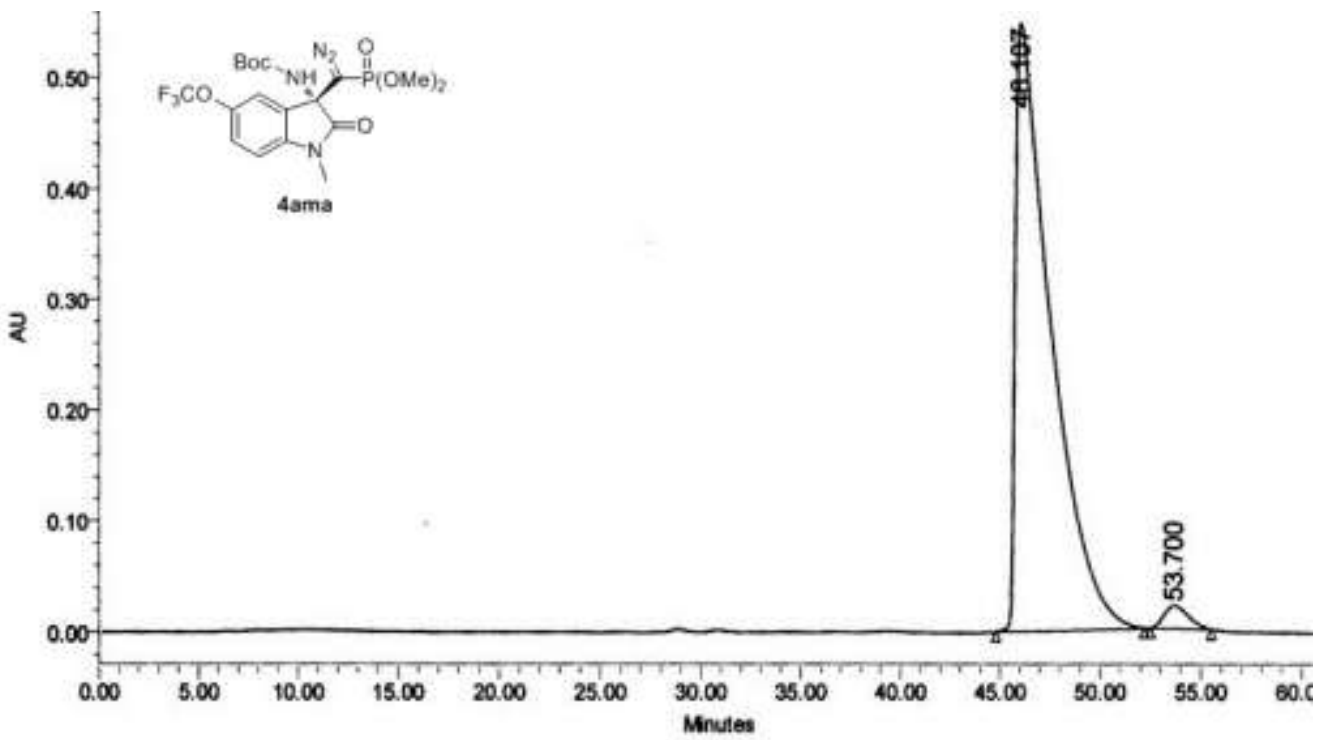

\begin{tabular}{|c|c|c|c|c|c|}
\hline & $\begin{array}{c}\text { RT } \\
(\mathrm{min})\end{array}$ & $\begin{array}{c}\text { Area } \\
(\mu \mathrm{V} / \mathrm{sec})\end{array}$ & $\%$ Area & $\begin{array}{c}\text { Height } \\
(\mu \mathrm{N})\end{array}$ & $\begin{array}{c}\% \\
\text { Height }\end{array}$ \\
\hline 1 & 46.107 & 67459805 & 97.54 & 548850 & 96.41 \\
\hline 2 & 53.700 & 1701143 & 2.46 & 20452 & 3.59 \\
\hline
\end{tabular}




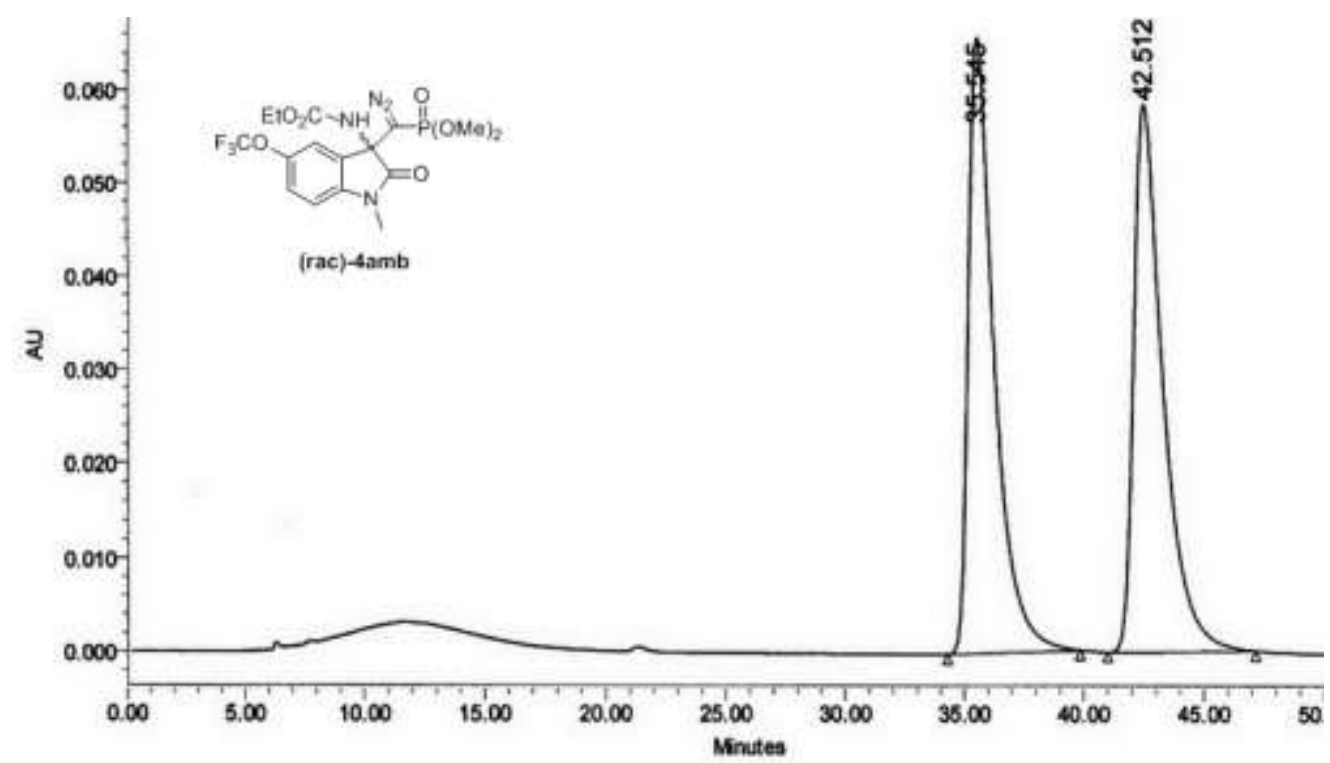

\begin{tabular}{|c|c|c|c|c|c|}
\hline & $\begin{array}{c}\text { RT } \\
(m i n)\end{array}$ & $\begin{array}{c}\text { Area } \\
\left(\mu N^{*} \text { sec }\right)\end{array}$ & $\%$ Area & $\begin{array}{c}\text { Height } \\
(\mu \mathrm{M})\end{array}$ & $\begin{array}{c}\% \\
\text { Height }\end{array}$ \\
\hline 1 & 35.545 & 4962158 & 50.04 & 65791 & 52.94 \\
\hline 2 & 42.512 & 4954901 & 49.96 & 58475 & 47.06 \\
\hline
\end{tabular}

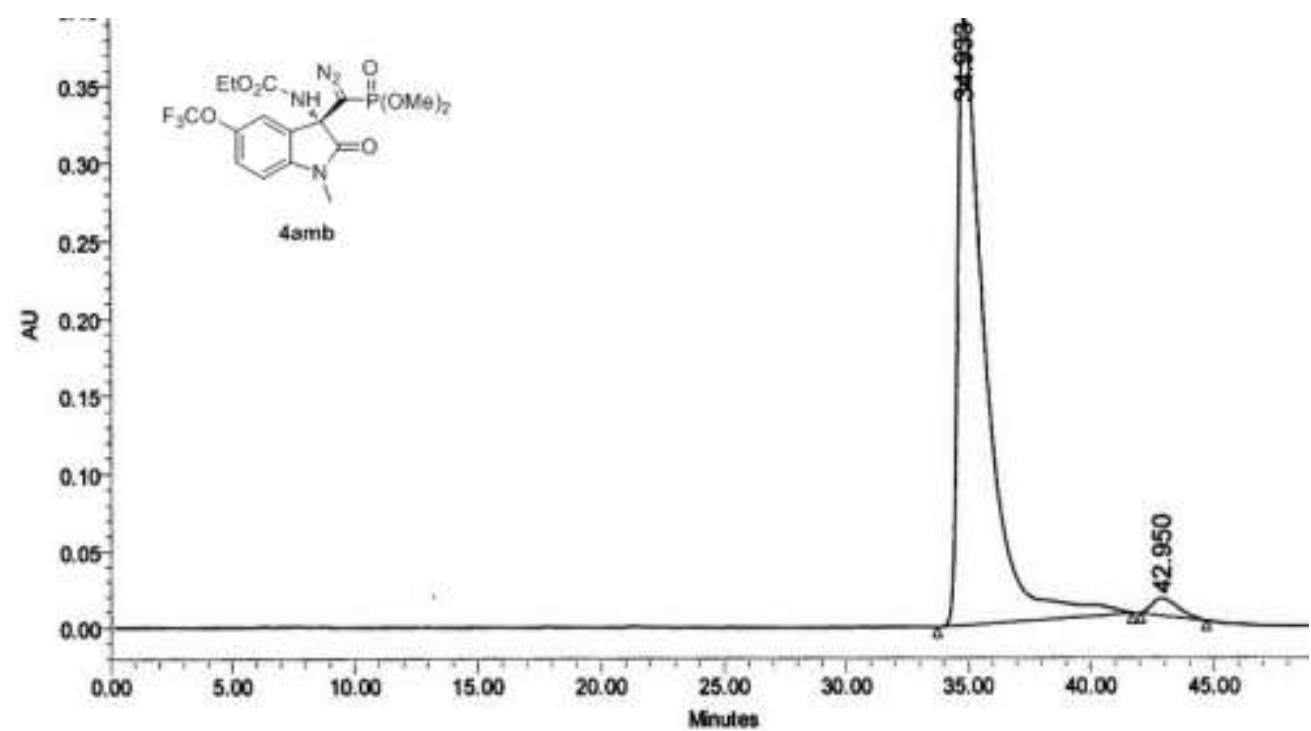

\begin{tabular}{|c|c|c|c|c|c|}
\hline & $\begin{array}{c}\text { Rा } \\
(\text { min })\end{array}$ & $\begin{array}{c}\text { Area } \\
(\mu \mathrm{N} \text { 'sec })\end{array}$ & \% Area & $\begin{array}{c}\text { Height } \\
(\mu \mathrm{V})\end{array}$ & $\begin{array}{c}\% \\
\text { Height }\end{array}$ \\
\hline 1 & 34.933 & 30912102 & 97.43 & 393153 & 97.22 \\
\hline 2 & 42.950 & 815462 & 2.57 & 11232 & 2.78 \\
\hline
\end{tabular}




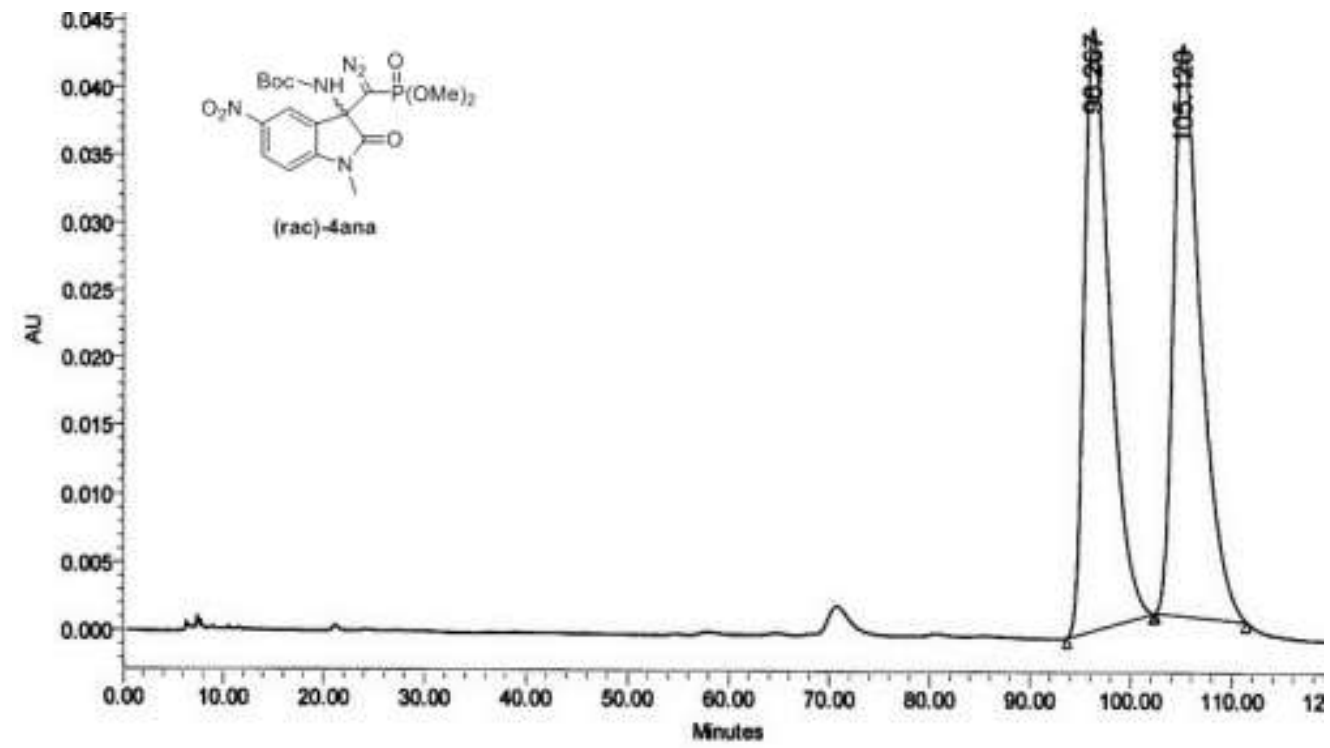

\begin{tabular}{|c|c|c|c|c|c|}
\hline & $\begin{array}{c}\text { Rा } \\
(\mathrm{min})\end{array}$ & $\begin{array}{c}\text { Area } \\
\left(\mu \mathrm{N}^{2} \mathrm{sec}\right)\end{array}$ & \% Area & $\begin{array}{c}\text { Height } \\
(\mu \mathrm{M})\end{array}$ & $\begin{array}{c}\% \\
\text { Height }\end{array}$ \\
\hline 1 & 96.267 & 7718194 & 49.66 & 44362 & 51.31 \\
\hline 2 & 105.120 & 7823376 & 50.34 & 42101 & 48.69 \\
\hline
\end{tabular}

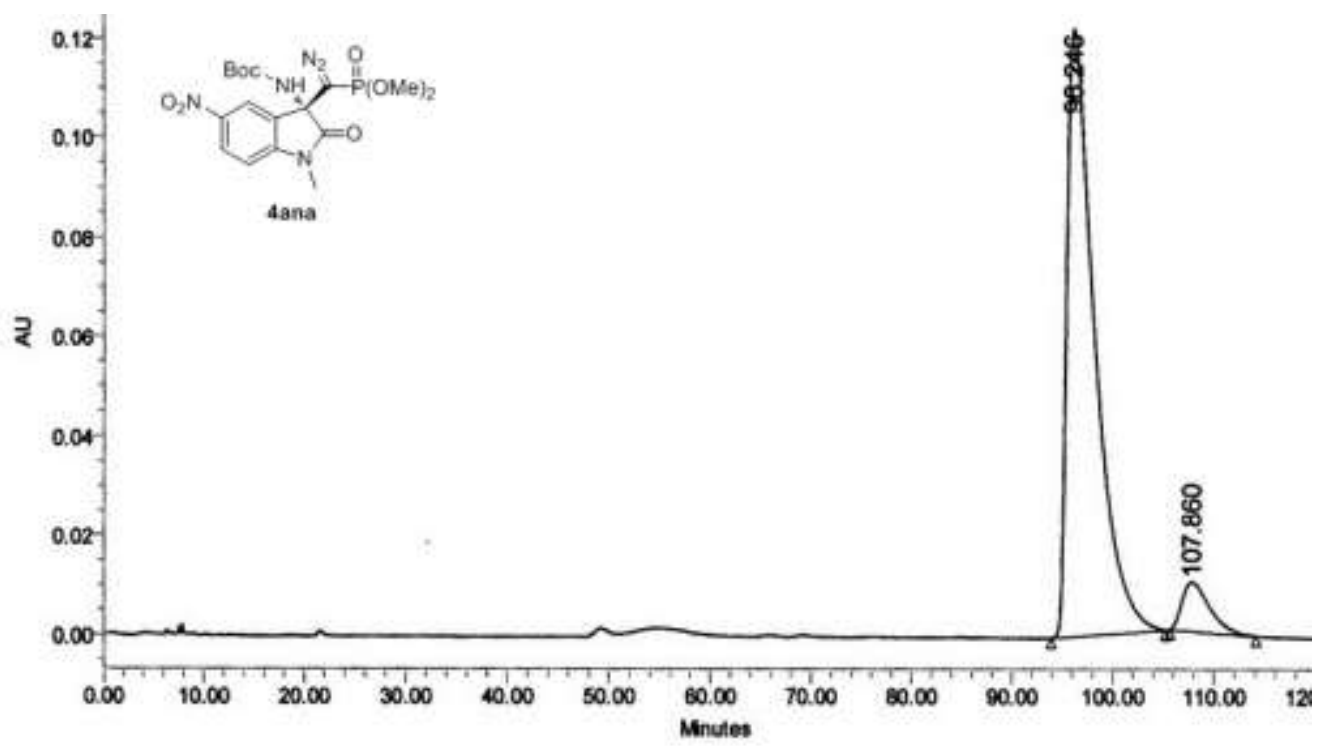

\begin{tabular}{|c|c|c|c|c|c|}
\hline & $\begin{array}{c}\text { RT } \\
(\mathrm{min})\end{array}$ & $\begin{array}{c}\text { Area } \\
(\mu \mathrm{N} \text { "sec })\end{array}$ & \% Area & $\begin{array}{c}\text { Height } \\
(\mu \mathrm{N})\end{array}$ & $\begin{array}{c}\% \\
\text { Height }\end{array}$ \\
\hline 1 & 96.246 & 23267492 & 92.66 & 122403 & 92.53 \\
\hline 2 & 107.860 & 1844010 & 7.34 & 9888 & 7.47 \\
\hline
\end{tabular}




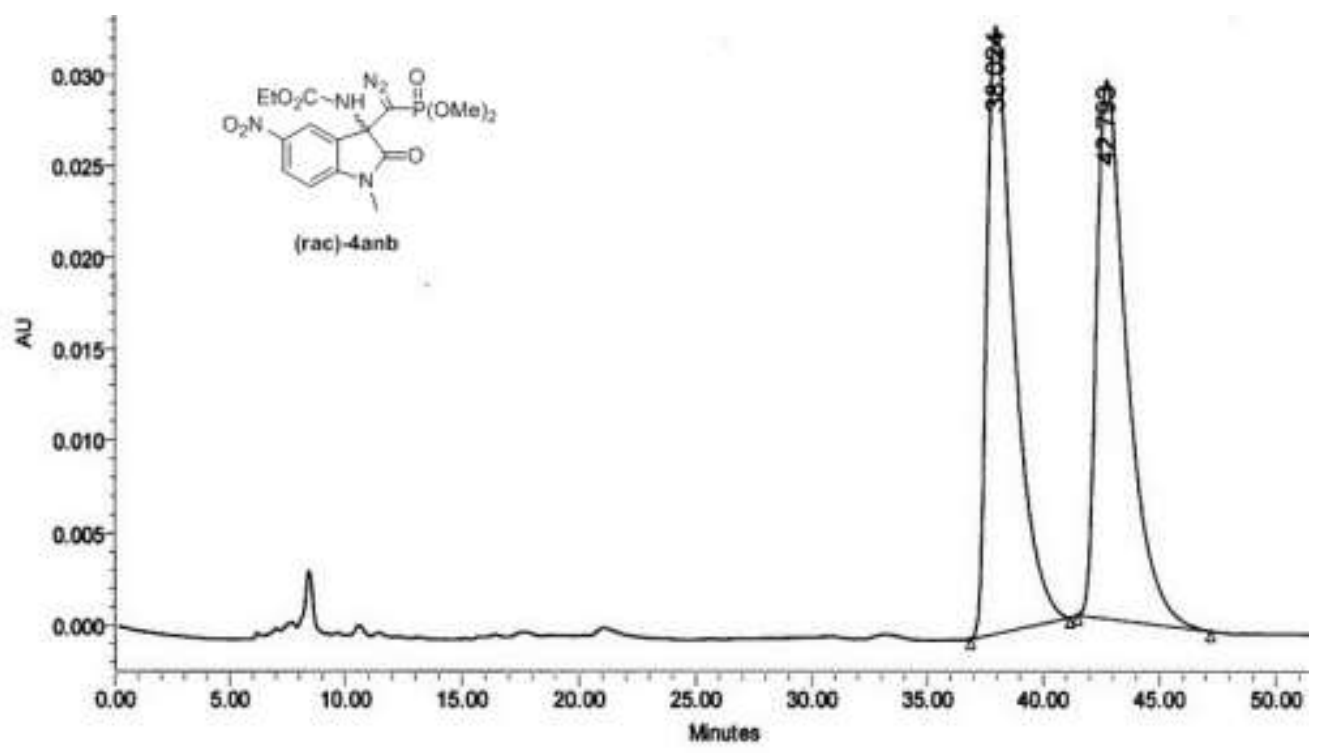

\begin{tabular}{|c|c|c|c|c|c|}
\hline & $\begin{array}{c}\text { RT } \\
(\mathrm{min})\end{array}$ & $\begin{array}{c}\text { Area } \\
\left(\mu \mathrm{V}^{*} \text { sec }\right)\end{array}$ & $\%$ Area & $\begin{array}{c}\text { Height } \\
(\mu \mathrm{M})\end{array}$ & $\begin{array}{c}\% \\
\text { Height }\end{array}$ \\
\hline 1 & 38.024 & 2688020 & 50.26 & 33086 & 52.99 \\
\hline 2 & 42.793 & 2660168 & 49.74 & 29353 & 47.01 \\
\hline
\end{tabular}

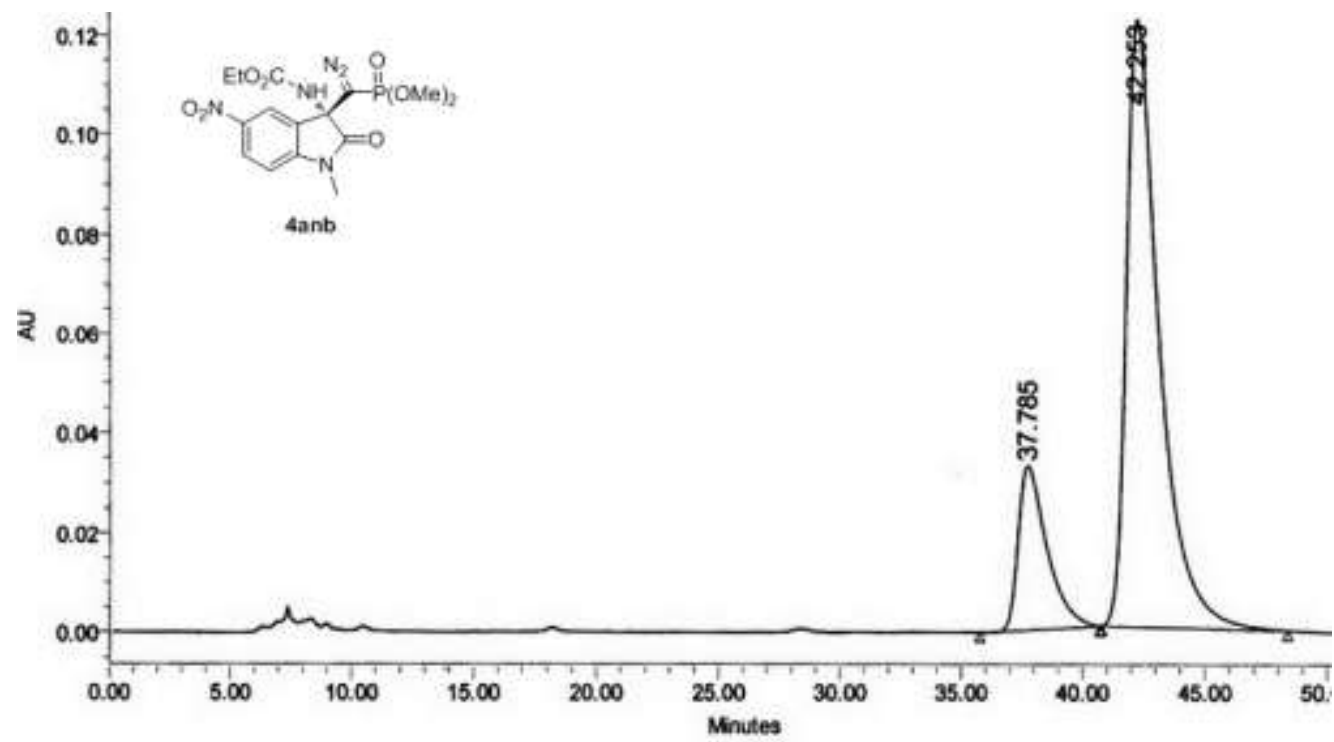

\begin{tabular}{|c|c|c|c|c|c|}
\hline & $\begin{array}{c}\text { RT } \\
(\mathrm{min})\end{array}$ & $\begin{array}{c}\text { Area } \\
(\mu \mathrm{N} \text { "sec })\end{array}$ & \% Area & $\begin{array}{c}\text { Height } \\
(\mu \mathrm{N})\end{array}$ & $\begin{array}{c}\% \\
\text { Height }\end{array}$ \\
\hline 1 & 37.785 & 2700847 & 19.19 & 32871 & 21.20 \\
\hline 2 & 42.253 & 11370683 & 80.81 & 122172 & 78.80 \\
\hline
\end{tabular}




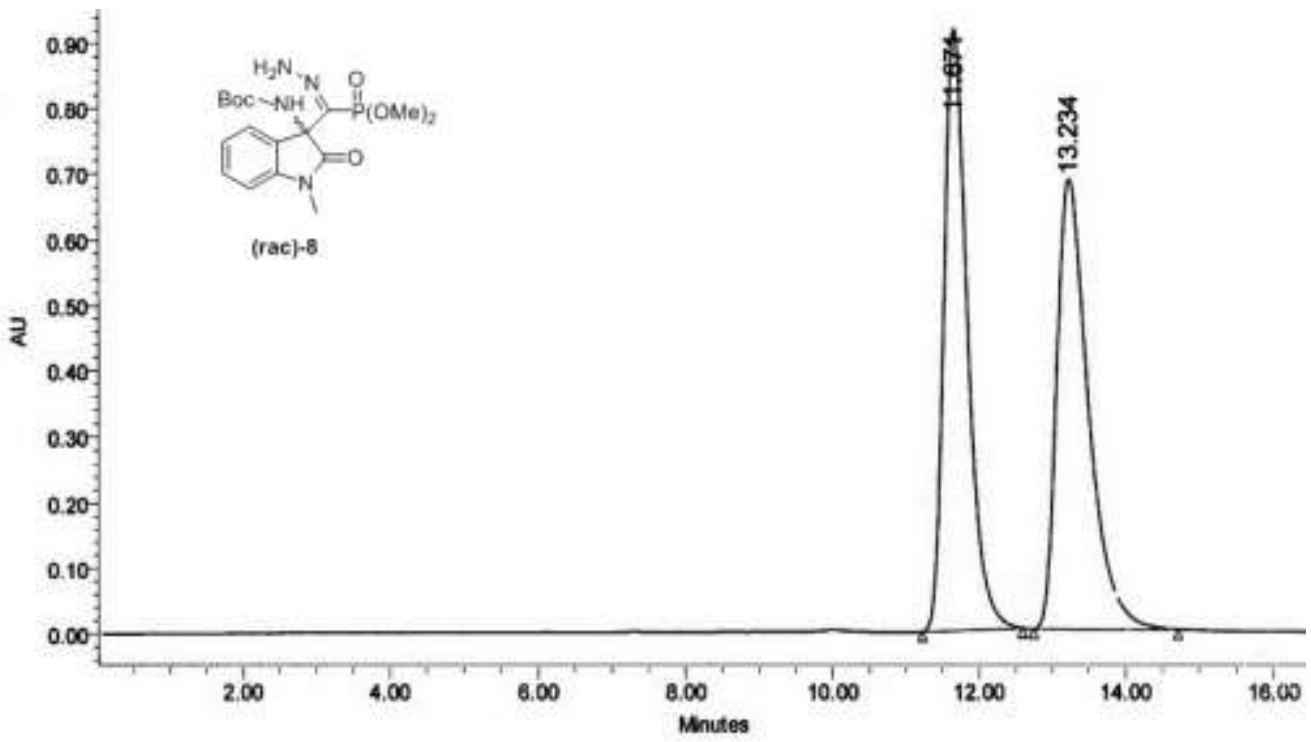

\begin{tabular}{|c|c|c|c|c|c|}
\hline & $\begin{array}{c}\text { RT } \\
(\mathrm{min})\end{array}$ & $\begin{array}{c}\text { Area } \\
(\mu \mathrm{N} \text { sec })\end{array}$ & \% Area & $\begin{array}{c}\text { Height } \\
(\mu \mathrm{V})\end{array}$ & $\begin{array}{c}\% \\
\text { Height }\end{array}$ \\
\hline 1 & 11.671 & 20444706 & 50.05 & 918334 & 57.30 \\
\hline 2 & 13.234 & 20403172 & 49.95 & 684375 & 42.70 \\
\hline
\end{tabular}

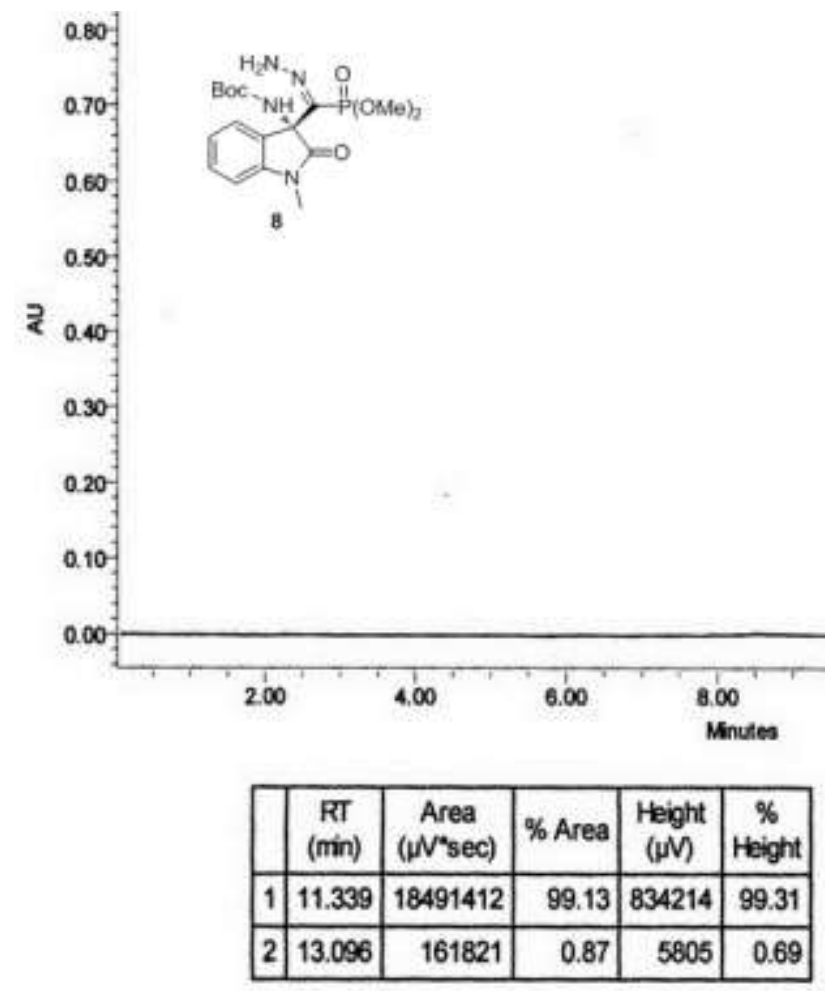




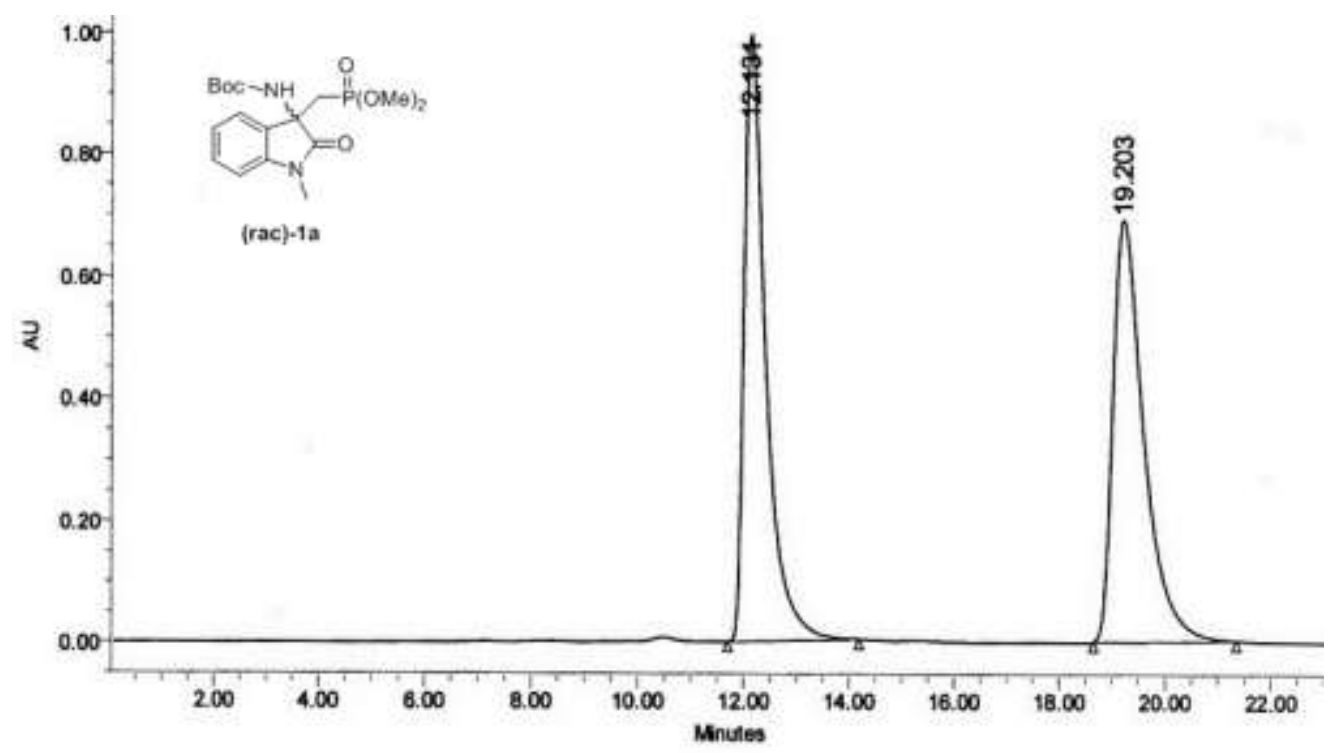

\begin{tabular}{|c|c|c|c|c|c|}
\hline & $\begin{array}{c}\text { RT } \\
(\text { min })\end{array}$ & $\begin{array}{c}\text { Area } \\
\left(\mu V^{\prime} \text { sec }\right)\end{array}$ & \% Area & $\begin{array}{c}\text { Height } \\
(\mu N)\end{array}$ & $\begin{array}{c}\% \\
\text { Height }\end{array}$ \\
\hline 1 & 12.131 & 28431661 & 50.27 & 997493 & 58.98 \\
\hline 2 & 19.203 & 28131554 & 49.73 & 693710 & 41.02 \\
\hline
\end{tabular}

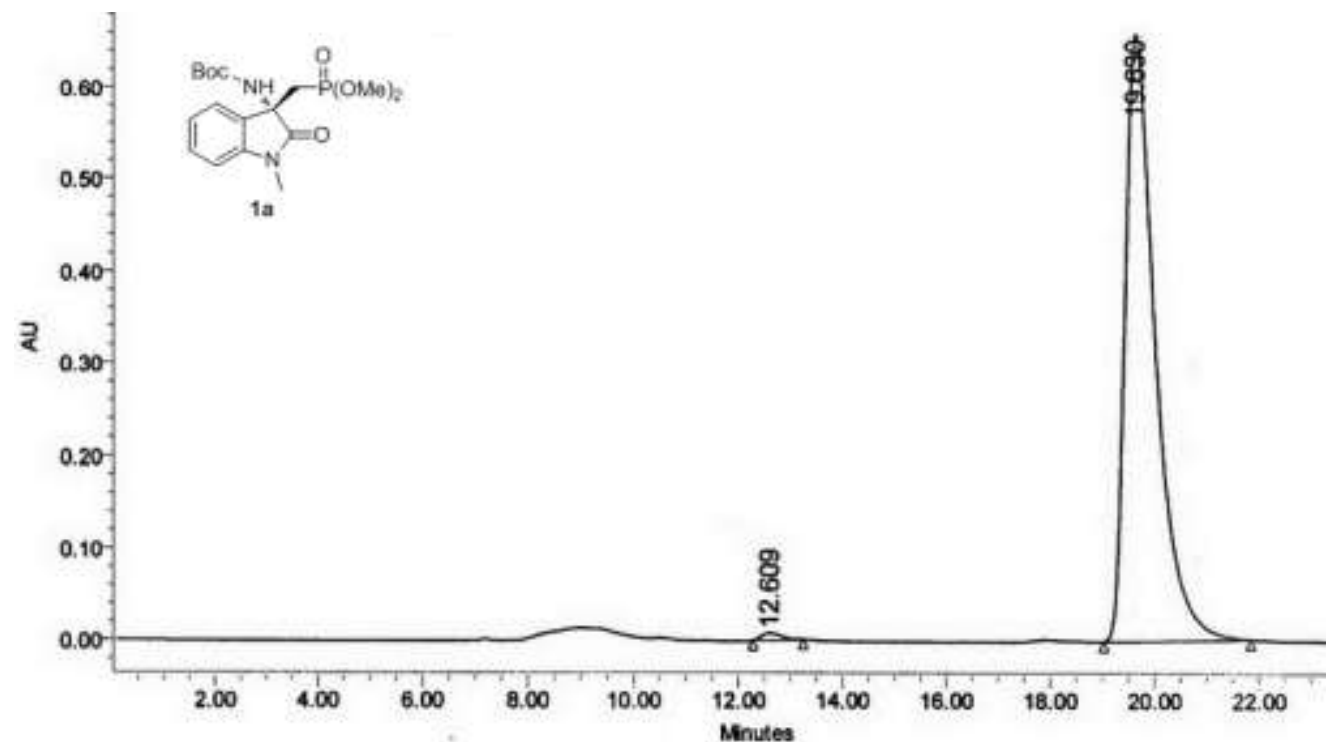

\begin{tabular}{|c|c|r|r|r|r|}
\hline & $\begin{array}{c}\text { RT } \\
(\mathrm{min})\end{array}$ & $\begin{array}{c}\text { Area } \\
\left(\mu \mathcal{N}^{*} \mathrm{sec}\right)\end{array}$ & \% Area & $\begin{array}{c}\text { Height } \\
(\mu \mathrm{N})\end{array}$ & $\begin{array}{c}\% \\
\text { Height }\end{array}$ \\
\hline 1 & 12.609 & 192984 & 0.72 & 7504 & 1.13 \\
\hline 2 & 19.630 & 26430985 & 99.28 & 659183 & 98.87 \\
\hline
\end{tabular}




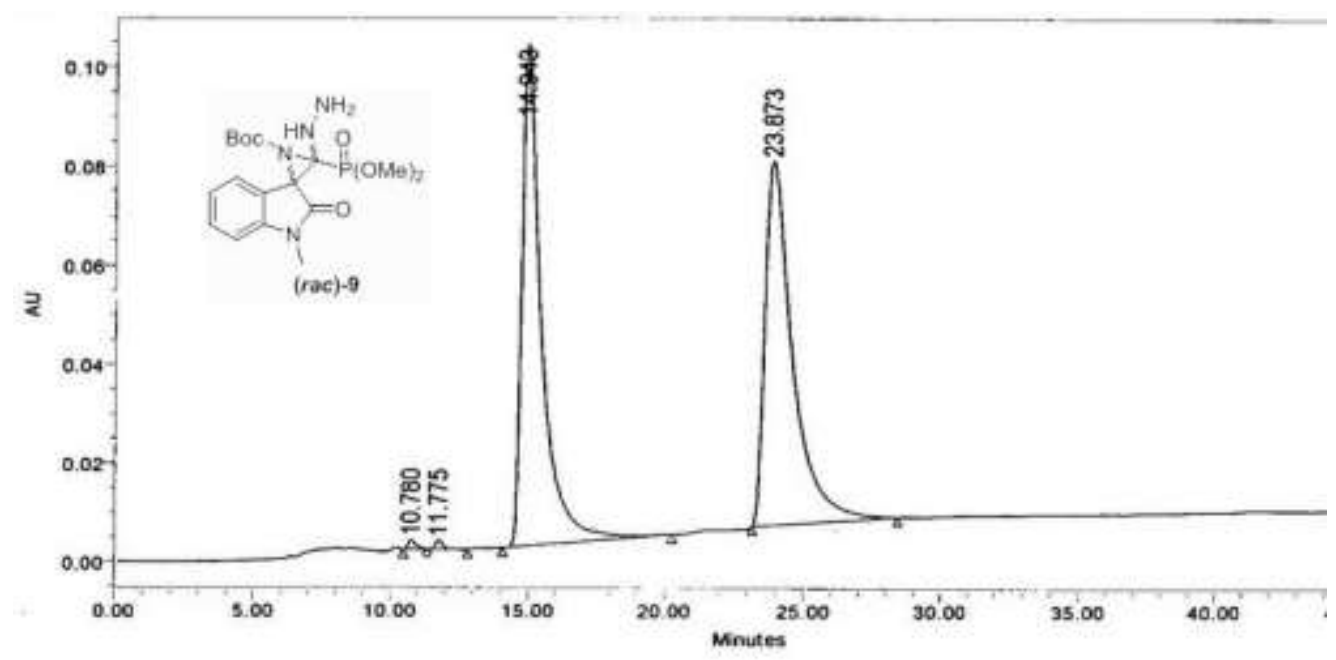

\begin{tabular}{|r|c|r|r|r|r|}
\hline & $\begin{array}{c}\text { RT } \\
(\mathrm{min})\end{array}$ & $\begin{array}{c}\text { Area } \\
\left(\mu \mathrm{N}^{*} \text { sec }\right)\end{array}$ & \% Area & $\begin{array}{r}\text { Height } \\
(\mu \mathcal{N})\end{array}$ & \multicolumn{1}{c|}{$\begin{array}{c}\% \\
\text { Height }\end{array}$} \\
\hline 1 & 10.780 & 37826 & 0.37 & 1963 & 1.10 \\
\hline 2 & 11.775 & 32155 & 0.31 & 1819 & 1.02 \\
\hline 3 & 14.943 & 5110071 & 49.65 & 101397 & 56.69 \\
\hline 4 & 23.873 & 5111339 & 49.67 & 73695 & 41.20 \\
\hline
\end{tabular}

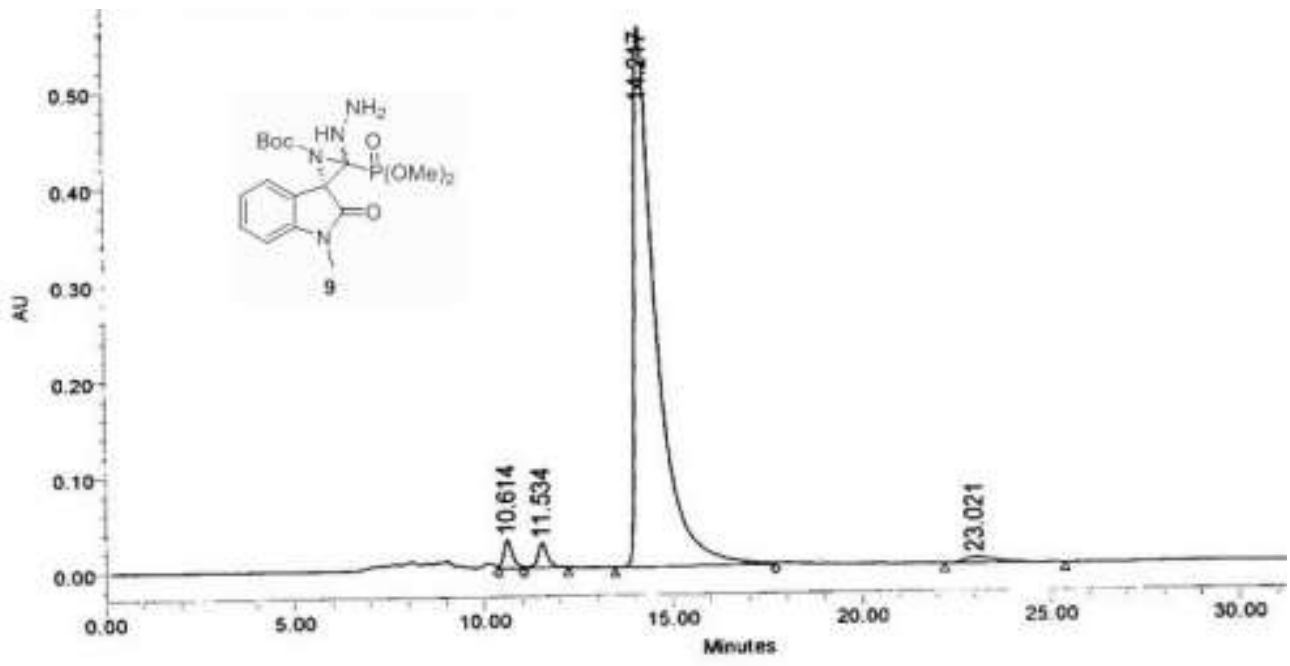

\begin{tabular}{|r|r|r|r|r|r|}
\hline & $\begin{array}{c}\text { RT } \\
(\mathrm{min})\end{array}$ & $\begin{array}{c}\text { Area } \\
\left(\mu \mathrm{N}^{\prime} \text { sec }\right)\end{array}$ & \% Area & $\begin{array}{c}\text { Height } \\
(\mu \mathrm{N})\end{array}$ & $\begin{array}{c}\text { \% } \\
\text { Height }\end{array}$ \\
\hline 1 & 10.614 & 481882 & 2.04 & 29917 & 4.80 \\
\hline 2 & 11.534 & 470669 & 1.99 & 26000 & 4.17 \\
\hline 3 & 14.217 & 22308003 & 94.40 & 560865 & 90.04 \\
\hline 4 & 23.021 & 371471 & 1.57 & 6117 & 0.98 \\
\hline
\end{tabular}

